\title{
IMAGING NEW ZEALAND'S CRUSTAL STRUCTURE USING AMBIENT SEISMIC NOISE RECORDINGS FROM PERMANENT AND TEMPORARY INSTRUMENTS
}

\author{
By
}

Yannik Behr

\author{
A thesis \\ submitted to Victoria University of Wellington \\ in fulfilment of the requirements for the degree of \\ Doctor of Philosophy \\ in Geophysics
}

Victoria University of Wellington

2011 



\section{Abstract}

We use ambient seismic noise to image the crust and uppermost mantle, and to determine the spatiotemporal characteristics of the noise field itself, and examine the way in which those characteristics may influence imaging results. Surface wave information extracted from ambient seismic noise using cross-correlation methods significantly enhances our knowledge of the crustal and uppermost mantle shear-velocity structure of New Zealand. We assemble a large dataset of three-component broadband continuous seismic data from temporary and permanent seismic stations, increasing the achievable resolution of surface wave velocity maps in comparison to a previous study.

Three-component data enables us to examine both Rayleigh and Love waves using noise cross-correlation functions. Employing a Monte Carlo inversion method, we invert Rayleigh and Love wave phase and group velocity dispersion curves separately for spatially averaged isotropic shear velocity models beneath the Northland Peninsula. The results yield first-order radial anisotropy estimates of $\sim 2 \%$ in the upper crust and up to $15 \%$ in the lower crust, and estimates of Moho depth and uppermost mantle velocity compatible with previous studies.

We also construct a high-resolution, pseudo-3D image of the shear-velocity distribution in the crust and uppermost mantle beneath the central North Island using Rayleigh and Love waves. We document, for the first time, the lateral extent of low shear-velocity zones in the upper and mid-crust beneath the highly active Taupo Volcanic Zone, which have been reported previously based on spatially confined 1D shear-velocity profiles. Attributing these low shear-velocities to the presence of partial melt, we use an empirical relation to estimate an average percentage of partial melt of $<4.2 \%$ in the upper and middle crust.

Analysis of the ambient seismic noise field in the North Island using plane wave beamforming and slant stacking indicates that higher mode Rayleigh waves can be detected, in addition to the fundamental mode. The azimuthal distributions of seismic noise sources inferred from beamforming are compatible with high near-coastal ocean wave heights in the period band of the secondary microseism $(\sim 7 \mathrm{~s})$. Averaged over 130 days, the distribution of seismic noise sources is azimuthally homogeneous, indicating that the seismic noise field is well-suited to noise cross-correlation studies. This is underpinned by the good agreement of our results with those from previous studies. The effective homogeneity of the seismic noise field and the large dataset of noise cross-correlation functions we here compiled, provide the cornerstone for future studies of ambient seismic noise and crustal shear velocity structure in New Zealand. 


\section{Acknowledgments}

I would like to thank my advisers Martha Savage, Stephen Bannister and most of all John Townend for always being approachable, for their constant support and their patient and thoughtful editing of this thesis. Their approach to science has taught me many things and I feel privileged to have studied under their supervision. I also want to express my gratitude to Michael Ritzwoller for hosting me in Boulder and to Fan-Chi Lin, Yingjie Yang and Morgan Moschetti for sharing their experience. Discussions with Lionel Carter, Martin Reyners, Tim Stern, Laura Brooks, Paul Grimwood and Wiebke Heise greatly helped to improve this thesis. I thank everyone who contributed to this work and in particular I want to thank Sonja Greve, Bill Fry, Kevin Buckley, John Hine, Melissa Bowen, Nick Mortimer, Mark Chadwick, Andrew Mellanby and Marc Wathelet. GeoNet and its staff have provided invaluable data and technical support, and this project would not have been possible without the funding from Victoria University of Wellington's Vice Chancellor's Strategic Research Scholarship and the Royal Society of New Zealand's Marsden Fund. I thank my student colleagues and most of all my former and present office mates, Sandra Bourgignon, Adam Carrizales, Adrian Benson, Zara Rawlinson and Carolin Boese for providing a pleasant and stimulating work environment. My discussions with Adrian on geophysics, geology and football were always enjoyable and often insightful. Long conversations with my brother Jonas and my good friend Moritz were motivating and have helped me with many technical difficulties. I also want to thank Mark Henderson for not resenting me for my bad driving skills.

It has been a lot of fun to live with Jan and Karly and I feel very lucky to have such good friends and flatmates. I dearly thank my parents Charly and Claudia for their unconditional support even at times when my decisions may have seemed incomprehensible. And, finally, I want to thank Rachel for all her love, patience and support over the last two years and especially the last two months. 


\section{Contents}

Abstract i

Acknowledgments iii

Contents $\quad$ v

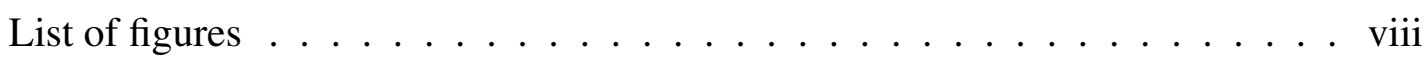

1. Introduction 1

1.1. Motivation and objectives . . . . . . . . . . . . . . . . . . . . . . . .

1.2. Tectonic setting .................... 4

1.3. Thesis outline . . . . . . . . . . . . . . 6

2. Methodology 13

2.1. Noise sources . . . . . . . . . . . . . . . . . . . 13

2.2. Turning noise into signal . . . . . . . . . . . . . . . . . 14

2.3. Processing . . . . . . . . . . . . . . . . 16

2.3.1. Pre-processing . . . . . . . . . . . . . . 18

2.3.2. Cross-correlation and velocity measurements . . . . . . . . . 20

2.3.3. Quality control ... . . . . . . . . . . . 23

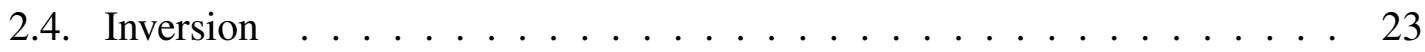

2.4.1. Surface wave tomography . . . . . . . . . . . . . 24

2.4.2. Inverting dispersion curves for shear velocity structure . . . . . 25

2.4.3. $1 \mathrm{D}$ and pseudo-3D shear velocity modelling . . . . . . . . . . 27

3. Rayleigh and Love wave phase velocity maps of New Zealand 31

3.1. Introduction . . . . . . . . . . . . . . . . . . . . 31

3.2. Datasets . . . . . . . . . . . . . . . 32

3.3. Resolution . . . . . . . . . . . . . . . . . 33

3.4. Results . . . . . . . . . . . . . . . . . . . . . 40

3.4.1. Rayleigh wave group velocities . . . . . . . . . . . . . 40

3.4.2. Rayleigh and Love wave phase velocities . . . . . . . . . . . . 41 
3.5. Discussion and conclusion . . . . . . . . . . . . . . . . 48

4. S-velocity structure of the Northland Peninsula 53

4.1. Introduction . . . . . . . . . . . . . . . . . . . 53

4.1.1. Data ....................... 55

4.1.2. Geology ................... 55

4.1.3. Previous studies . . . . . . . . . . . . . 57

4.2. Method . . . . . . . . . . . . . . . . . . 57

4.2.1. Preprocessing and velocity measurements . . . . . . . . . . 57

4.2.2. Error analysis . . . . . . . . . . . . . . . . 58

4.2.3. Inversion for $\mathrm{S}$-velocity profiles . . . . . . . . . . . . 59

4.3. Results . . . . . . . . . . . . . . . . . . . . . . . . . 60

4.4. Discussion and conclusion . . . . . . . . . . . . . . 66

5. Ambient noise tomography of the central North Island 73

5.1. Introduction . . . . . . . . . . . . . . . . . . . . . . . . . 74

5.2. Noise source characteristics . . . . . . . . . . . . . . . . . . 76

5.3. Error analysis . . . . . . . . . . . . . . . . . . 81

5.3.1. Instrument responses and timing errors . . . . . . . . . . 82

5.3.2. Dispersion curve uncertainties . . . . . . . . . . . . . . . 84

5.4. Tomography . . . . . . . . . . . . . . . . . . . . . . . . 89

5.5. Pseudo-3D inversion . . . . . . . . . . . . . . . . . . 94

5.5.1. Methodology . . . . . . . . . . . . . . 94

5.5.2. Model parameterisation . . . . . . . . . . . . . 98

5.5.3. Evaluation ................... 99

5.6. Discussion . . . . . . . . . . . . . . . . . . . . 111

5.6.1. Implications for the presence of melt . . . . . . . . . . . . . 111

5.6.2. Implications for TVZ evolution . . . . . . . . . . . . . . . 117

5.7. Conclusion . . . . . . . . . . . . . . . . . 119

$\begin{array}{ll}\text { 6. Beamforming and slant stacking } & 121\end{array}$

6.1. Introduction . . . . . . . . . . . . . . . . . 121

6.1.1. Plane wave beamforming . . . . . . . . . . . . . 123

6.1.2. Slant stacking . . . . . . . . . . . . . . . . 125

6.1.3. Datasets . . . . . . . . . . . . . . 128

6.2. Results . . . . . . . . . . . . . . . . . . 128

6.2.1. Mt. Taranaki deployment . . . . . . . . . . . . . . . . 128

6.2.2. START deployment . . . . . . . . . . . . . . . . . . 133 
6.3. Discussion and conclusion . . . . . . . . . . . . . 145

7. Summary of key results and opportunities for further research $\quad 149$

$\begin{array}{ll}\text { A. Appendix } & 153\end{array}$

A.1. Data access ........................ 153

A.2. Grid computing . . . . . . . . . . . . . . . . 155

A.3. ObsPy .............................. 156

$\begin{array}{ll}\text { B. Response function database } & 161\end{array}$

B.1. CNIPSE . . . . . . . . . . . . . . . . . . 161

B.2. WCNIPSE . . . . . . . . . . . . . . . . . . 168

B.3. NORD . . . . . . . . . . . . . . . . . . . 170

B.4. RF2004 . . . . . . . . . . . . . . . . . . 171

B.5. GeoNet stations . . . . . . . . . . . . . . . . 173

$\begin{array}{ll}\text { Bibliography } & 174\end{array}$ 


\section{List of Figures}

1.1. Example of surface wave dispersion and sensitivity kernels . . . . . . 2

1.2. Scopus search results on "ambient seismic noise". . . . . . . . . . . . . . 3

1.3. New Zealand's main tectonic features and basement geology . . . . . . . 4

1.4. Rayleigh and Love wave phase velocity maps at $8 \mathrm{~s}$ period . . . . . . 8

1.5. 1D S-velocity model comparison . . . . . . . . . . . . . . 9

1.6. Pseudo-3D shear velocity model of the central North Island . . . . . . . . 10

1.7. Beamformer results for the $6 \mathrm{~s}$ period averaged over 130 days . . . . . . . 11

2.1. Synthetic cross-correlation functions . . . . . . . . . . . . . . . 17

2.2. Simplified processing scheme after Bensen et al. [2007] . . . . . . . . . 18

2.3. Effect of spectral and temporal normalization . . . . . . . . . . . . 19

2.4. Effect of phase-matched filtering . . . . . . . . . . . . . . . . 22

2.5. Voronoi cell example . . . . . . . . . . . . . . . . . . . . . 27

2.6. Saturation value for the Neighbourhood algorithm . . . . . . . . . . . 28

3.1. Seismic broadband stations used for ambient noise tomography . . . . . . 34

3.2. Operating timespans of temporary deployments . . . . . . . . . . . 35

3.3. Synthetic input model for checkerboard test . . . . . . . . . . . . . 36

3.4. Results checkerboard test . . . . . . . . . . . . . . . . . 37

3.5. Azimuthal path coverage . . . . . . . . . . . . . . . 38

3.6. Resolution analysis . . . . . . . . . . . . . . . . . . 39

3.7. Number of velocity measurements for Rayleigh and Love wave phase velocities . . . . . . . . . . . . . . . . 44 40

3.8. Rayleigh wave group velocity maps Lin et al. . . . . . . . . . . . . . . . 42

3.9. Rayleigh wave group velocity maps . . . . . . . . . . . . . . . . 43

3.10. New Zealand overview map . . . . . . . . . . . . . . . . . . . 44

3.11. Rayleigh wave phase velocity maps . . . . . . . . . . . . . . . 47

3.12. Love wave phase velocity maps . . . . . . . . . . . . . . . . . 49

3.13. New Zealand's basement geology . . . . . . . . . . . . . . . 50

4.1. Correlation functions of the NORD deployment . . . . . . . . . . 56 
4.2. SNR vs. period for cross-correlations . . . . . . . . . . . . . . . 61

4.3. Dispersion curves . . . . . . . . . . . . . . . . . . 61

4.4. 1D Rayleigh wave inversion between TIKO and MATA and TIKO and WCZ 63

4.5. 1D Rayleigh and Love wave inversion between TIKO and MATA . . . . . 64

4.6. Moho depth histograms . . . . . . . . . . . . . . . . . . . . . . 65

4.7. 1D inversion with fixed layer thickness . . . . . . . . . . . . . . 67

4.8. Estimates of radial anisotropy _ . . . . . . . . . . . . . . 68

5.1. Seismic stations North Island . . . . . . . . . . . . . . . . . . 77

5.2. Array response START . . . . . . . . . . . . . . . . . . . 79

5.3. Beamforming results $22 / 2 / 2001 \ldots \ldots \ldots$

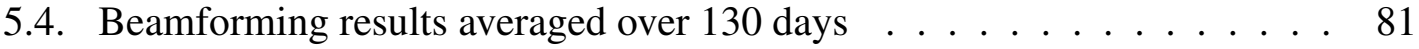

5.5. Polarity test . . . . . . . . . . . . . . . 83

5.6. Timing correction . . . . . . . . . . . . . . . 85

5.7. Signal-to-noise ratio statistics . . . . . . . . . . . . . . . . . 86

5.8. Average dispersion curves for temporary deployments . . . . . . . . . . . 87

5.9. SNR proxy curves . . . . . . . . . . . . . . . . . 88

5.10. RMS difference between causal and acausal part of cross-correlation functions . . . . . . . . . . . . . . . . . . 89

5.11. Dispersion curves with error bars at $-38.5^{\circ} / 176.0^{\circ} \ldots \ldots$. . . . . . 90

5.12. Central North Island cross-correlation functions ～. . . . . . . . . . . . . 90

5.13. SNR vs period . . . . . . . . . . . . . . . . . . 92

5.14. Misfit statistics for different damping parameters . . . . . . . . . . 93

5.15. Surface wave maps for the $6 \mathrm{~s}$ period . . . . . . . . . . . . . . 95

5.16. Surface wave maps for the $10 \mathrm{~s}$ period . . . . . . . . . . . . . . 96

5.17. Comparison of Rayleigh wave radial and vertical components . . . . . . . 97

5.18. Sensitivity kernels . . . . . . . . . . . . . . . . . . . . . 97

5.19. 1D S-velocity models . . . . . . . . . . . . . . . . . 100

5.20. Geological map of the central North Island . . . . . . . . . . . . . . 101

5.21. Spatial distribution of misfit values from 1D inversions . . . . . . . . . . 101

5.22. Pseudo-3D S-velocity model . . . . . . . . . . . . . . . . . . 102

5.23. 2D S-velocity profile along the CNIPSE/WCNIPSE line . . . . . . . . 103

5.24. 2D S-velocity profile along strike of the TVZ . . . . . . . . . . . . . 105

5.25 . Isosurface at $3.1 \mathrm{~km} / \mathrm{s} \ldots \ldots \ldots$. . . . . . . . . . . . . 106

5.26. Standard deviation for 3D model . . . . . . . . . . . . . . . . . 108

5.27. 2D S-velocity profile along the CNIPSE/WCNIPSE line from Love wave group velocities . . . . . . . . . . . . . . . . . . . . . 109 
5.28. 2D S-velocity profile along strike of the TVZ from Love wave group velocities . . . . . . . . . . . . . . . . . . . 110

5.29. 2D section through the S-velocity model from Love wave phase velocities 110

5.30. Partial melt in relation to inclusion geometry . . . . . . . . . . . . . . . . 112

5.31. Comparison of S-velocity profile with MT-study . . . . . . . . . . . . . . 114

5.32. Location of S-velocity profile and MT-profile . . . . . . . . . . . . . 115

5.33. Comparison of S-velocity profile with active source studies . . . . . . . 118

6.1. Mt. Taranaki deployment map . . . . . . . . . . . . . . . . . . . . . . 129

6.2. START deployment map . . . . . . . . . . . . . . . . . . . . . 130

6.3. Beamformer results for Mt. Taranaki . . . . . . . . . . . . . . . . 131

6.4. Virtual common shot point gather Taranaki . . . . . . . . . . . . . 134

6.5. Slant stack for the Taranaki deployment . . . . . . . . . . . . . . 135

6.6. 1D S-velocity models from slant stack dispersion curves . . . . . . . . 136

6.7. Beamforming results $22 / 2 / 2001$ at 6 s period . . . . . . . . . . . . . 138

6.8. Beamforming results $3 / 3 / 2001$ at 6 s period . . . . . . . . . . . . . . 139

6.9. Beamforming results $3 / 3 / 2001$ at 8 s period . . . . . . . . . . . . . . 139

6.10. Mean ocean wave period for $22 / 2 / 2001$ and $3 / 3 / 2001 \ldots$. . . . . . . . 140

6.11. Beamforming results averaged over 130 days at $6 \mathrm{~s}$ period . . . . . . . 140

6.12. Beamforming results averaged over 130 days at $8 \mathrm{~s}$ period . . . . . . . 142

6.13. Virtual common shot point gather for START . . . . . . . . . . . . . . 142

6.14. Slant stack for the START deployment . . . . . . . . . . . . . . . . . . 143

6.15. 1D S-velocity models from slant stack dispersion curves: fundamental and first higher mode . . . . . . . . . . . . . . . . . . . . . 144

6.16. 1D S-velocity models from slant stack dispersion curves: fundamental and second higher mode . . . . . . . . . . . . . . . . . . . 147

A.1. Webservice scheme . . . . . . . . . . . . . . . . . . . 154

A.2. Cross-correlation processing scheme . . . . . . . . . . . . . . 157

A.3. Runtime for computing cross-correlations . . . . . . . . . . . . . . 157

A.4. ObsPy example: processing . . . . . . . . . . . . . . . . . 160

A.5. Obspy example: data retrieval . . . . . . . . . . . . . . . . . 160 



\section{Introduction}

\subsection{Motivation and objectives}

Seismic surface waves propagate near the earth's surface and their amplitudes, therefore, decay with distance $r$ from the source proportionally to $1 / \sqrt{r}$ (rather than $1 / r$ for body waves), which usually makes surface waves an earthquake seismogram's dominant feature (Figure 1.1). The preponderance of surface waves found expression in early seismological terminology, which described the surface wave train as the "main shock" [see Dahlen and Tromp, 1998]. However, it was not until the works of Rayleigh [1885] and Love [1911] that these large-amplitude waves were identified as surface waves. They showed that elliptically polarized waves in the vertical plane (Rayleigh waves) and linearly polarized waves in the horizontal plane (Love waves), decaying with depth, were solutions to the equation of motion. Love [1911] further showed that surface waves are dispersive, meaning that their propagation velocity depends on their wavelength. As the depth of maximum displacement, or sensitivity, of surface waves increases with period, so does, in general the surface wave's velocity [e.g. Stein and Wysession, 2003; Aki and Richards, 2002, and Figure 1.1]. Sensitivity is not restricted to a specific depth but extends over a depth range, and as a result surface wave velocities at discrete periods represent the seismic properties integrated over the corresponding depth range. Their high sensitivity to the shear-velocity of the medium they travel through has thus underpinned many studies of shear-velocity structure between the earth's surface and the lower mantle [e.g. Gutenberg, 1924; Kuo et al., 1962; Forsyth, 1975; Mitchell, 1984; Mocquet et al., 1989; Villaseñor et al., 2001; Shapiro et al., 2004; Debayle, 2004].

Inferring earth structure from surface waves generated by earthquakes suffers several shortcomings [see Ritzwoller and Levshin, 1998; Shapiro et al., 2005, and references therein]. With any method based on passive source signals, the retrievable lateral resolution depends on the locations of seismometers and earthquakes: the former are generally constrained to on-shore or near-shore positions and the latter to subduction zones and continental and oceanic rifts. This typically results in a nonuniform and somewhat arbitrary 

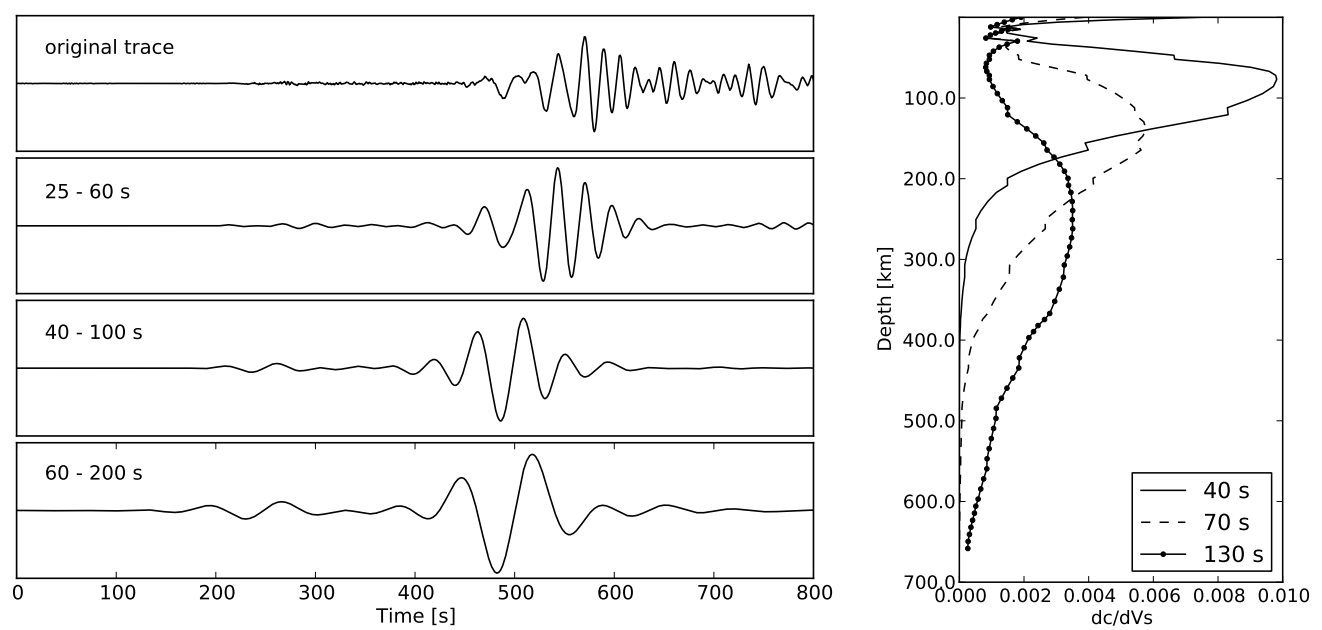

Figure 1.1 Left panels: Vertical component seismogram of the Darfield earthquake, which occured close to Christchurch, New Zealand at 16:36 on 3 September 2010 UTC, recorded at RAO (Raoul, Kermadec Islands) on 3 September 2010 between 16:36 and 16:50 UTC. The upper left panel shows the unfiltered seismogram, with the surface waves representing the strongest part of the signal. The panels below show the seismogram filtered between 25 and $60 \mathrm{~s}, 40$ and $100 \mathrm{~s}$, and 60 and $200 \mathrm{~s}$ period. Surface waves at longer periods arrive earlier, and are therefore faster, than those at shorter periods. Right panel: Phase velocity sensitivity to shear velocity with respect to depth for Rayleigh waves at $40 \mathrm{~s}, 70 \mathrm{~s}$, and $130 \mathrm{~s}$ period calculated for the Preliminary Earth Model [PREM; Dziewonski and Anderson, 1981]. The longer the period, the greater the depth of highest sensitivity.

sampling of the study area. The use of active sources partially overcomes this problem but is more expensive, and common active sources are usually not sufficiently energetic to seismically image the same depths as surface waves from earthquakes. Another problem affecting both active- and passive-source surface wave studies is that short-period surface waves are attenuated more strongly than long-period surface waves. Reliable surface wave observations from teleseismic earthquakes at periods shorter than $\sim 20 \mathrm{~s}$ have been made [e.g. Zielhuis and Nolet, 1994; Huang, 2003] but are in general rare [Yao et al., 2006]. Since the longer-period surface waves have little sensitivity to depths shallower than $\sim 30 \mathrm{~km}$, upper and mid-crustal structures are poorly constrained.

It has been known since the 1950s that surface wave information can be extracted from ambient seismic noise using cross-correlation techniques [Aki, 1957]. As ambient seismic noise is globally pervasive, its use as a source of energy for seismic imaging largely eliminates the problem of a non-uniform distribution of earthquakes. However, as we discuss in more detail in Chapters 2, 5 and 6, the strength and spatial distribution of noise sources is also not strictly homogeneous, an important constraint which has to be considered when extracting surface wave information from the ambient seismic noise.

As noise sources commonly lie closer to the recording instruments than earthquakes, sur- 


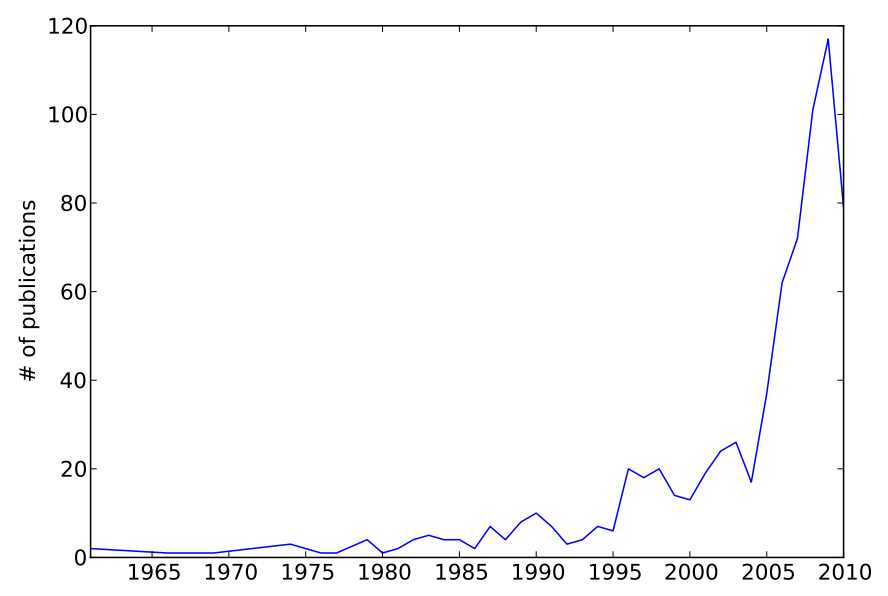

Figure 1.2 Number of publications in earth and planetary sciences resulting from a Scopus search (http://www.scopus.com/) for the keywords "ambient seismic noise", "seismic noise" and "ambient noise" (accessed 23 October 2010). The full Scopus search string is: (TITLE-ABS-KEY("ambient seismic noise”) OR TITLE-ABS-KEY(“seismic noise”) OR TITLE-ABS-KEY(“ambient noise”)) AND ( LIMITTO(SUBJAREA,'EART” )).

face waves extracted from seismic noise experience less attenuation, and so reliable surface wave observations can therefore potentially be made for periods as short as $1 \mathrm{~s}$ on regional scales [e.g. Brenguier et al., 2007] and less than $1 \mathrm{~s}$ on local scales [e.g. Louie, 2001; Di Giulio et al., 2006]. Conversely, the maximum observable period of surface waves extractable from seismic noise is typically restricted to less than $\sim 50$ s by the observing seismic array's maximum station spacing, as we discuss in more detail in Chapter 2. This means that the primary use for such data is for inferring crustal and uppermost mantle shear-velocity structure.

Ambient noise related research has become increasingly popular over the last decade, with several studies now being published each month (see Figure 1.2). Due to their dispersive nature, seismic surface waves, like ocean gravity waves, travel at two different speeds: the velocity of the wave packet (group velocity) and the velocity of a single wave (phase velocity) [e.g. Stein and Wysession, 2003; Aki and Richards, 2002]. Because the initial phase of the source as well as the number of cycles a wave travelled through, do not affect the group velocity, measuring the group velocity is easier than measuring the phase velocity [e.g. Ritzwoller and Levshin, 1998]. Consequently, many early studies on extracting surface wave velocities from noise cross-correlation functions focused on the measurement of Rayleigh wave group velocities [e.g. Shapiro et al., 2005; Gudmundsson et al., 2007; Lin et al., 2007]. Yao et al. [2006] were the first to measure Rayleigh wave phase velocities from noise cross-correlations. Lin et al. [2008] extended the analysis of noise 

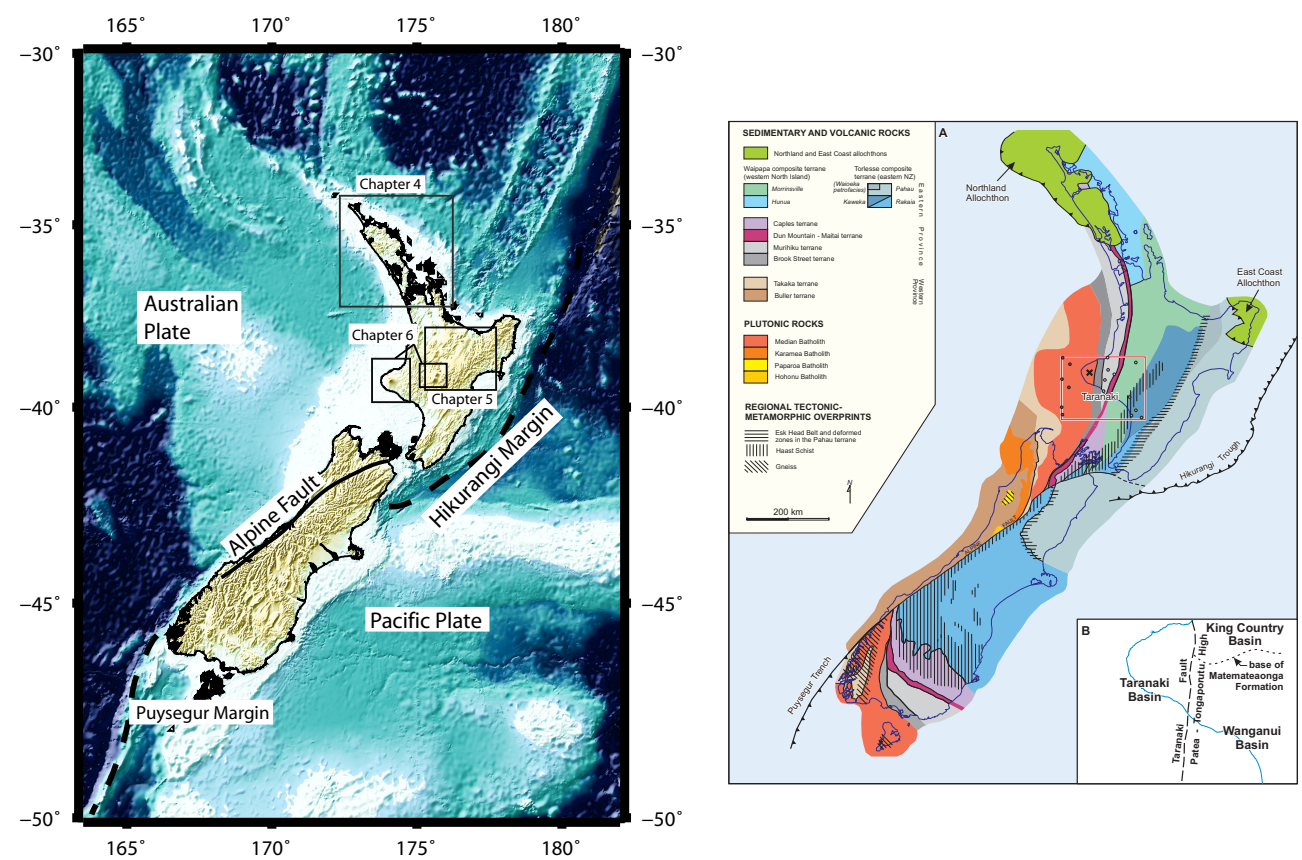

Figure 1.3 Left panel: main tectonic features in New Zealand. Right panel: New Zealand's basement geology [Mortimer, 2004].

cross-correlations to horizontal component seismograms, measuring Rayleigh and Love wave phase velocities.

\subsection{Tectonic setting}

New Zealand lies on the boundary between the Pacific and Australian plate whose relative motion changes from subduction, off the east coast of the North Island (Hikurangi margin), to oblique-convergent strike-slip in the western South Island (Alpine fault) and subduction with the opposite polarity (Puysegur margin) southwest of the South Island (Figure 1.3). The collision of continental crust in the South Island results in the continuing uplift of the Southern Alps [Walcott, 1998] and the strike-slip component motion along the Alpine fault has resulted in a total offset of 440-470 km [Sutherland, 1999b]. The transition from dominantly strike-slip plate motion in the South Island to oblique subduction in the North Island is characterized by a system of strike-slip faults - the Marlborough Fault System — [Eberhart-Phillips and Bannister, 2010] and by a plate interface at depth that appears to be locked [Reyners, 1998; Wallace, 2004; Wallace et al., 2009]. 
The seismogenic zone of the subducting Pacific plate lies at depths as shallow as $\sim 15 \mathrm{~km}$ beneath the eastern North Island and and extends down to $\sim 300 \mathrm{~km}$ beneath the western North Island [Reyners et al., 2006]. Subduction-related processes largely dominate the tectonics of the North Island, causing deformation and partial melting in the overlying crust and upper mantle [e.g. Harrison and White, 2004; Stern et al., 2006; Seward et al., 2009; Rowland et al., 2010]. The Taupo Volcanic Zone (TVZ) in the central North Island is the current center of tectonomagmatic activity, rifting apart by up to $15 \mathrm{~mm} / \mathrm{yr}$ at its northern end and $\sim 7 \mathrm{~mm} / \mathrm{yr}$ further south [Wallace, 2004]. The TVZ is further characterised by high heat flow [average heat flux of $700 \mathrm{~mW} / \mathrm{m}^{2}$; Bibby et al., 1995] and active andesitic, basaltic and rhyolitic volcanism $\left[15-20,000 \mathrm{~km}^{3}\right.$ of volcanic deposits not older than 2 Myr; Wilson et al., 1995; Houghton et al., 1995].

As recently renewed by Mortimer [2004], New Zealand's basement geology can be subdivided into several regional terranes, accreted and intruded when New Zealand was part of the Cambrian to early Cretaceous convergent continental margin of Gondwana (Figure 1.3). Plutonic basement rocks are located to the west of New Zealand and at the southernmost end of the South Island with the Median Batholith being the largest plutonic terrane. The central and eastern South and North Island are dominated by the Rakaia Terrane and Pahau Terrane mainly consisting of greywacke and overprinted in parts by the Haast Schist. The Waipapa terranes on the western and northwestern North Island also comprise greywacke but contain larger amounts of volcanoclastic material than the terranes further east [Adams et al., 2009].

Detailed knowledge of crustal structures in general, and seismic properties, in particular, is important for understanding how localized phenomena observed on the surface, such as volcanism and faulting, are connected to deeper-seated larger-scale tectonic processes like subduction or convective systems operating in the mantle [e.g. Stern et al., 2006; Reyners and Eberhart-Phillips, 2009]. Many of these processes are associated with melt and fluid migrations from the mantle into the crust. Shear velocities are more sensitive to small fractions of melt and fluids in a medium than compressional waves [Schmeling, 1985] but, for reasons discussed in Chapter 5, are more difficult to infer using more traditional seismological methods.

The main scientific question we address in this study is that of what surface waves from ambient seismic noise in New Zealand tell us about crustal shear-velocity structures, about the distribution of seismic noise sources, and the reliability of this information. 


\subsection{Thesis outline}

The work described here was conducted as part of a study of the ambient seismic noise field of New Zealand and its use in seismic imaging, led by Victoria University of Wellington and GNS Science and funded by the Royal Society of New Zealand's Marsden Fund. We further contributed to two projects described in Appendix A.1 and A.2, the former funded by the Research and Education Advanced Network New Zealand Ltd and the latter by New Zealand's Earthquake Commission.

This thesis consists of a sequence of five largely stand-alone chapters (excluding this chapter and a short summary chapter), each with its own technical abstract and introduction suitable for publication. Chapter 4 has been published [Behr et al., 2010] as well as parts of Chapter 5 [Behr et al., 2011]. The order of Chapters 3, 4 and 5 represents our train of thought and demonstrates how we can retrieve increasingly detailed information on crustal seismic structure from ambient seismic noise. Chapter 6 has a somewhat different emphasis from these chapters in that it primarily discusses the characteristics of the ambient seismic noise field of the North Island.

Most of the work in this project and all of the work presented in this thesis has been conducted by me (Yannik Behr) unless explicitly stated otherwise in the text (see Section 6.2.1). Two chapters (Chapters 4 and 5) have been published entirely or in part and therefore have been written using the first person plural, as it is common practice for publications involving more than one author. To be consistent with Chapters 4 and 5 I have chosen to write the rest of the thesis in the first person plural instead of the first person singular. This images also the fact that my supervisor's guidance and advice has been inextricably linked to the success of this project.

\section{Chapter 2: Methodology}

Chapter 2 provides an overview of the recent and rapidly evolving literature and describes the processing and inversion methods used throughout this study. The main processing step is the cross-correlation of long durations of ambient seismic noise records between every possible pair of stations in a seismic array. It has been shown in several theoretical and applied studies that the cross-correlation of seismic noise between two seismographs yields an estimate of the Green's function between the two stations. In practice, the surface wave part of the Green's function can be constructed most reliably. We therefore focus on measuring Rayleigh and Love wave surface wave dispersion curves from crosscorrelation functions of vertical and horizontal component seismograms and then follow 
a procedure commonly used in teleseismic studies to infer shear-velocity structure from surface waves.

\section{Chapter 3: Rayleigh and Love wave phase velocity maps of New Zealand}

In a pilot study of ambient noise tomography in New Zealand, Lin et al. [2007] measured Rayleigh wave group velocities from noise cross-correlations between 42 permanent seismic broadband stations in the GeoNet network (http://www.geonet.org.nz), in order to construct surface wave velocity maps at several discrete periods. GeoNet is a project to build and operate the geological hazard monitoring system in New Zealand. It is part of GNS Science and funded by New Zealand's Earthquake Commission.

Lin et al.'s [2007] results exhibited striking correlations with known geological features, with low surface wave velocities mainly corresponding to sedimentary and volcanic deposits and high velocities mainly to igneous and metamorphic regions. In Chapter 3 we extend Lin et al.'s [2007] study by amalgamating data from several temporary deployments with data from the permanent seismic network in New Zealand (left panel in Figure 1.4). We measure Rayleigh wave phase and group velocities and Love wave phase velocities between stations of the extended dataset. The main advantage of phase velocities over group velocities is their higher sensitivity to deeper structures [Dahlen and Zhou, 2006]. Rayleigh and Love waves are most sensitive to vertically and horizontally polarized shear waves, respectively, and are, at identical periods, sensitive to different depths. As we show in Chapter 3, surface wave maps from Rayleigh and Love waves reveal similar structures overall but differ in details. Figure 1.4 shows the surface wave phase velocity maps for Rayleigh and Love waves at $8 \mathrm{~s}$ period together with the locations of temporary and permanent seismic stations included in this study. Dispersion curves measured between the stations on the Northland Peninsula and in the central North Island are analysed in Chapters 4 and 5 respectively in terms of crustal and uppermost mantle shear velocity structure.

\section{Chapter 4: Shear-velocity structure of the Northland Peninsula, New Zealand}

In Chapter 4 we describe the measurement of Rayleigh and Love wave phase and group velocities between two pairs of seismometers that were part of a temporary deployment on the Northland Peninsula in the northwest of New Zealand's North Island. In view 

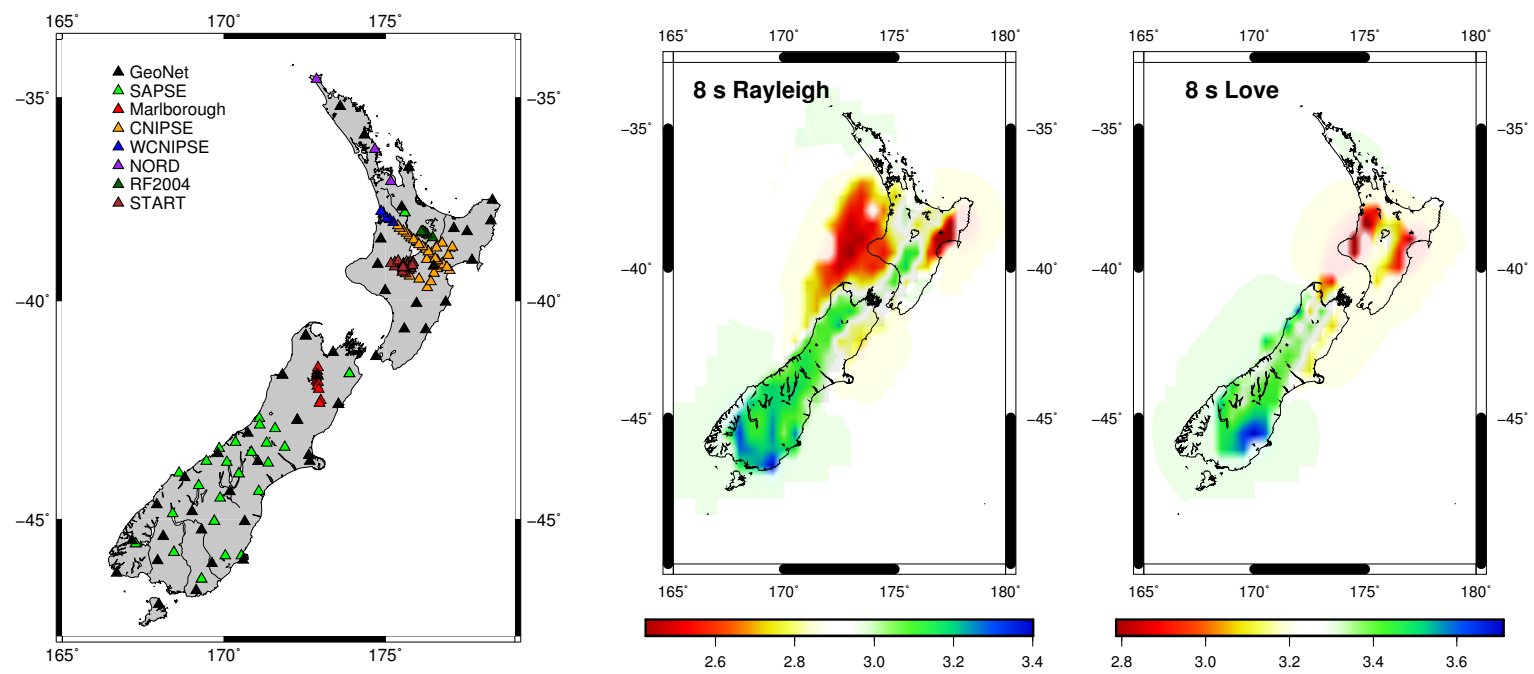

Figure 1.4 Left panel: locations for of temporary deployment and permanent seismic stations used in this study. A larger version of this panel can be found in Chapter 3. Middle and right panel: lateral surface wave phase velocity distribution for Rayleigh and Love waves at $8 \mathrm{~s}$ period. Surface wave phase velocities were inferred from noise cross-correlations between the seismic stations shown on the left panel.

of the results of several theoretical studies indicating that spatially homogeneous noise fields are required for extracting surface wave information from noise cross-correlations, the ambient noise field and the seismic network geometry in Northland are both clearly sub-optimal [Snieder, 2004; Sánchez-Sesma and Campillo, 2006]. Our results demonstrate the robustness of the surface wave information obtainable from ambient noise crosscorrelations even under less than theoretically ideal conditions.

Inferring shear velocity structure from surface wave velocities is a highly nonlinear problem [e.g. Dunkin, 1965], so we invert the measured dispersion curves for path averaged shear velocity versus depth profiles, employing a Monte-Carlo grid search method known as the Neighbourhood Algorithm [Sambridge, 1999a, b]. This constitutes a considerable improvement over the surface wave maps presented in Chapter 3, which represent only the integrated velocity over specific depth ranges. Our results agree well with those from a previous study of the same region involving receiver functions and surface waves from teleseismic earthquakes (Figure 1.5) [Horspool et al., 2006]. Additionally, by comparing 1D shear velocity profiles inferred from Rayleigh and Love wave dispersion curves, we are able to make a first-order estimate of radial anisotropy in the crust. The results of this chapter have been published in the Journal of Geophysical Research [Behr et al., 2010]. 


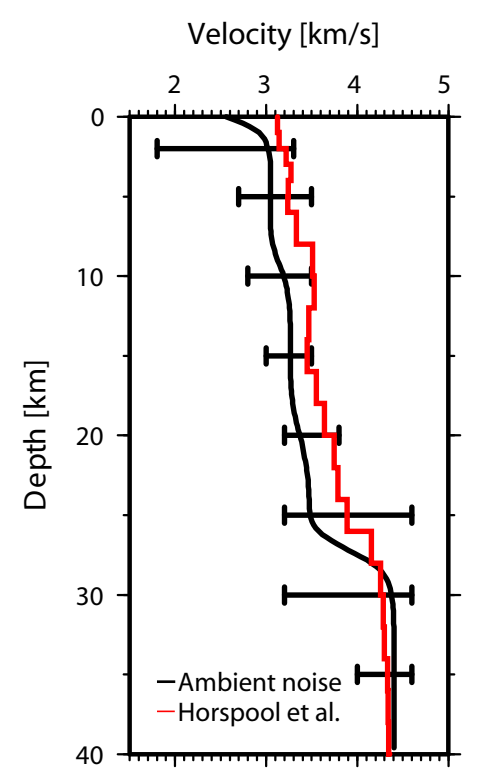

Figure 1.5 Path-averaged 1D shear-velocity profiles beneath the Northland Peninsula, inferred from surface-waves using noise cross-correlations (black line) and compared to the averaged results of Horspool et al. [2006] (red line). The error bars mark the range over which the Neighbourhood algorithm found models that were in agreement with the surface wave observations.

\section{Chapter 5: Ambient noise tomography of the central North Island using temporary and permanent seismograph data}

In Chapter 5 we interpolate between the surface wave maps presented in Chapter 3 at successive periods to construct new dispersion curves at each point in a $0.25^{\circ} \times 0.25^{\circ}$ grid spanning the central North Island of New Zealand. Using the Monte Carlo method introduced in Chapter 4 we then calculate 1D shear velocity profiles at each of the grid points and combine the results to form a pseudo-3D volume (Figure 1.6). The results show zones of pronounced low velocities in the upper and middle crust ( $\sim-15 \mathrm{~km}$ depth), which correlate spatially with several presumed source regions of rhyolitic volcanism in the Taupo Volcanic Zone, the locus of continental rifting and volcanic activity in the central North Island.

As the first pseudo-3D crustal shear-velocity model of the central North Island, our results complement previous geophysical studies that mostly focused on the compressionalwave velocity and resistivity structure [e.g. Bibby et al., 1995; Stratford and Stern, 2006; Heise et al., 2010, and references therein]. When interpreted in conjunction with high lower-crustal compressional-wave velocities observed in active and passive source studies [Harrison and White, 2004; Reyners et al., 2006; Stern and Benson, 2011], low lowercrustal shear-velocities are consistent with high fractions of partial melt. This observation 


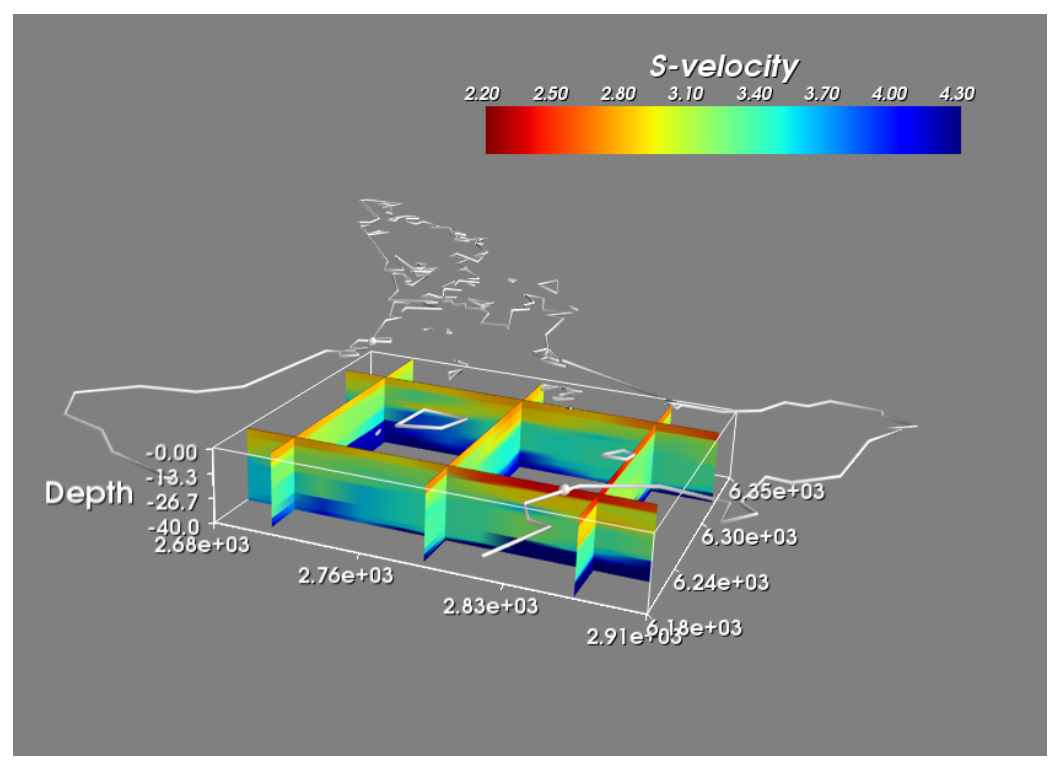

Figure 1.6 Five profiles through the pseudo-3D shear-velocity model inferred for the central North Island from Rayleigh wave phase and group velocities. Coordinates are in New Zealand Map Grid (NZMG).

is compatible with several volcanological and tectonic models of the central North Island [Rowland et al., 2010; Pulford and Stern, 2004]. The results of this chapter have been published in parts [Behr et al., 2011].

\section{Chapter 6: Beamforming and slant stack analysis of ambient noise data for noise field and structural characterisation}

The physical relationship between the distribution of ambient noise sources and the reliability of the information obtainable from noise cross-correlations has not yet been fully established [e.g. Tsai, 2010; Forghani and Snieder, 2010]. Identifying the strength, spectral characteristics and spatial distribution of seismic noise sources is the first step in understanding the information contained in seismic noise records. We show in Chapter 6 , using a plane wave beamforming method, that the source regions of ambient noise recorded in the central North Island, correlate with near-coastal ocean wave heights taken from a regional wave-action-model of New Zealand [Gorman et al., 2003a, b].

We also applied slant stacking [Chapman, 1978] to a group of noise cross-correlation functions between seismic stations of two temporary deployments on the North Island. This method is particularly useful for identifying coherent phases in the ambient seismic noise field from noise cross-correlation functions between seismic stations of small to medium aperture arrays ( $<100 \mathrm{~km}$ stations spacing) [Louie, 2001; Gouédard et al., 


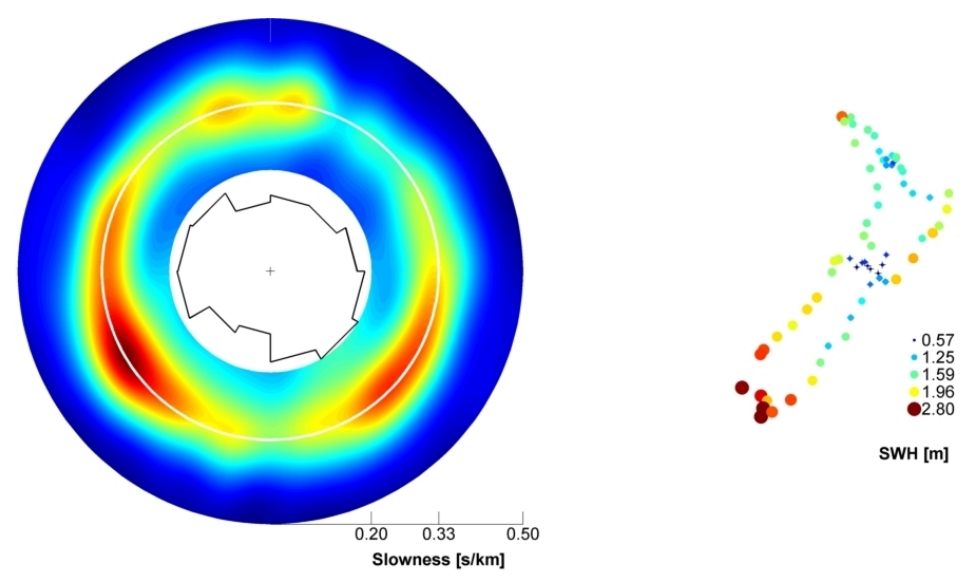

Figure 1.7 Left panel: plane wave beamforming results using seismic noise recordings filtered at $6 \mathrm{~s}$ and average over 130 days. The ocean wave energy flux averaged over the same 130 days is shown in the middle. Right panel: significant wave height at the $50 \mathrm{~m}$ isobath taken from a regional wave action model and averaged over the same 130 days.

2008]. Our results from plane wave beamforming [Brooks et al., 2009a] and slant stacking demonstrate that in addition to fundamental mode Rayleigh waves, higher-mode Rayleigh wave signals are also present in the seismic noise wavefield. However, probably due to interference effects, we find that higher modes cannot be clearly distinguished within the period band of the secondary microseism.

\section{Appendix A}

In the course of this study, several technical challenges were encountered and overcome in computing noise cross-correlations: large data volumes; slow processing; and heterogeneous recording conditions.

The analysis of long-duration (month- to year-long) continuous seismic data sampled at high rates requires special tools for querying and transferring data from data management centers. In collaboration with staff at GeoNet, the data management center for earth science data in New Zealand, we developed a web-service (Appendix A.1) that allows us to automatically and rapidly download continuous seismic records of any length from GeoNet, making full use of New Zealand's high-speed network infrastructure.

Due to the large volumes of data involved in ambient noise analysis, many steps of the processing are computationally expensive. However, the processing scheme is what computer scientists call "embarrassingly parallel", meaning that it involves a number of subprocesses that do not depend on one another and can be executed separately. We therefore 
developed methods of parallelizing the computation of noise cross-correlations and running them on a grid of 230 desktop computers, operated by the School of Engineering and Computer Science at Victoria University of Wellington (Appendix A.2). This resulted in the computations being completed up to 40-times faster than on a single processor desktop computer [Townend et al., 2009a].

The inclusion of temporary deployment data into our dataset as we did, requires the conversion of records originally stored in several different data formats into a consistent format. We have made use of and contributed to an open-source software project — "ObsPy" - that provides seismologists with tools addressing common seismological tasks, such as requesting data from data management centers or converting between different data formats [Appendix A.3; Beyreuther et al., 2010].

\section{Appendix B}

A further problem arising when combining data from different instrument types is incorrect or missing instrument response information. In Chapter 5 we describe a method to find seismic records from stations with incorrect instrument response functions. Since this also poses a problem for many other studies, we have compiled a list of instrument response functions used in this study. 


\section{Methodology}

This chapter provides a general overview of the theoretical basis and analytical approach underlying the thesis as a whole. We summarize the key topics in the light of recent results and provide further details where necessary in later chapters.

\subsection{Noise sources}

The sources for seismic noise differ for different period bands. As described by Bonnefoy-Claudet et al. [2006], noise at long periods ( $>1 \mathrm{~s}$ period) is produced by fluctuations of ambient conditions such as changes in air pressure or by sea waves hitting coastlines. For periods of $1-0.2 \mathrm{~s}$, the dominant sources can be either natural or artificial, while for periods shorter than $0.2 \mathrm{~s}$ most seismic noise is caused by traffic, industrial machinery, human footsteps, etc.

We focus in this study on noise dominated by waves whose periods exceed $1 \mathrm{~s}$, as this is the most persistent and energetic component of the noise field and is therefore most suitable for imaging geological structure from the surface down to the uppermost mantle [e.g. Yao et al., 2008; Moschetti et al., 2010]. Noise spectra at periods longer than $1 \mathrm{~s} \mathrm{ex-}$ hibit two characteristic peaks: the primary microseism at $\sim 14$ s period and the secondary microseism at $\sim 7$ s period [Petersen, 1993; Berger, 2004]. Longuet-Higgins [1950] described that the secondary microseism is generated by nonlinear interference effects of ocean waves travelling in opposite directions. Based on this theory, Friedrich et al. [1998] proposed that the primary microseism is mainly caused by the interaction of ocean waves with the near-coastal seafloor, whereas the secondary microseism is generated by the interference of incoming and reflected waves in the proximity of coastlines. Kedar et al. [2008] compared theoretical noise amplitudes calculated with Longuet-Higgins' [1950] theory and based on output from a wave action model with noise amplitudes at several seismometers around the Atlantic sea. The agreement between the times of peaks in the theoretical calculations and those observed at seismometers was striking, and implied the main source for the recorded secondary microseism to be in the North Atlantic. This 
shows that, whether the source regions of ambient seismic noise are located near coastlines or in the deep oceans, is in parts still controversial, as we discuss in more detail in Chapter 6.

\subsection{Turning noise into signal}

Aki [1957] demonstrated the feasibility of recovering surface wave information from ambient noise cross-spectra. It was later shown by Claerbout [1968] that a medium's Green's function, - that is, its impulse response function - could be recovered using crosscorrelation of noise. Duvall et al. [1993] and Rickett and Claerbout [2000] applied this principle to recover the Green's function between surface points on the sun by using random fluctuations in the observed solar spectrum.

The first decade of the twenty-first century has seen a resurgence of interest in random wavefields. Weaver and Lobkis [2001b] showed in experiments with ultrasound and thermal noise that the Green's function of an aluminium block can be measured from the cross-correlation of diffuse wavefields. This inspired a series of experiments into extracting useful information from seismic noise records by cross-correlation. Shapiro and Campillo [2004] were able to measure the dispersion curves of Rayleigh waves travelling between station pairs from the cross-correlation function of one monthlong intervals of seismic noise recorded at several stations in the USA. Shapiro et al. [2005] applied the same method to one month-long noise recordings at stations in California, and used the resulting dispersion curves to compute lateral surface wave velocity distributions at discrete periods of 7.5 and $15 \mathrm{~s}$. Their results correlated well with known geological features, with slow velocities in the surface wave maps corresponding to sedimentary basins and faster velocities to igneous regions. Both seismological studies took only noise data with periods longer than one second into account.

Following these promising results, a number of research groups began applying this technique to different areas. In the last five years, the method has been shown to be feasible over a broad range of scales spanning continental $(\sim 1000 \mathrm{~km})$ [Yang et al., 2007; Bensen et al., 2008; Stehly et al., 2009], regional ( $\sim 100 \mathrm{~km})$ [Kang and Shin, 2006; Lin et al., 2007; Li et al., 2010; Gallego et al., 2010], and local length scales ( $<10 \mathrm{~km})$ [Brenguier et al., 2007; Sabra et al., 2005; Stachnik et al., 2008].

While many studies have now demonstrated that useful and reasonable information can be extracted from the cross-correlations of ambient seismic noise, the causal relationship between noise cross-correlations for different ambient conditions and the Green's function 
has not been fully established. Sánchez-Sesma and Campillo [2006] derived the relation between the cross-correlation of random wavefields in a medium and the Green's function by employing the concept of equipartioning, meaning that in a modal representation of the wavefield every mode carries the same amount of energy. Tsai [2010] pointed out that seismic wavefields are typically not equipartitioned, and described conditions that depart from the requirement of equipartioning but still allow the retrieval of parts of the Green's function. In particular, Tsai [2010] attributed the dominance of fundamental mode surface wave modes in noise cross-correlation functions to the fact that these modes are primarily excited by near-surface noise sources and are therefore close to being equipartitioned. Higher order modes (including modes contributing to body waves) are usually less excited by near-surface sources and are accordingly further away from an equipartitioned state. Nevertheless, higher order surface waves and P-waves have been shown to be present in ambient seismic noise correlations [e.g. Roux et al., 2005; Harmon et al., 2007; Brooks et al., 2009a].

Snieder [2004] used a stationary phase argument to derive the reconstruction of the Green's function from the cross-correlation of random scalar wavefields recorded at two receivers and emitted by random scatterers surrounding the receivers. The stationary phase approximation assumes that phases vary slowly and therefore interfere constructively around a stationary point. Anywhere else they vary rapidly and interfere destructively [Aki and Richards, 2002]. Wapenaar [2006] demonstrated theoretically the reconstruction of the Green's function in the case of a one-sided distribution of random scatterers. Snieder et al. [2010] extended the concept of retrieving a medium's impulse response from the cross-correlation of random field fluctuations to a larger class of linear scalar systems which satisfy a generalized form of the acoustic wave equation. Amongst these fields are potential fields, such as electromagnetic fields, but also quantum fields, as the Schrödinger equation falls into this class of linear scalar systems.

Tsai [2009] and Yao and Van Der Hilst [2009] derived a plane wave formulation of noise cross-correlations assuming noise sources emitting harmonic wave pulses of amplitude $\rho$ and angular frequency $\omega$, recorded at two seismic stations. With this approach the crosscorrelation $C_{x y}$ of the wavefield recorded at station $x$ with the wavefield recorded at station $y$ can be expressed as

$$
C_{x y}(\Delta t, \omega, W)=\int W\left(\Delta t_{d}\right) \rho\left(\Delta t_{d}, \omega\right) \cos \left[\omega\left(\Delta t-\Delta t_{d}\right)\right] d \Delta t_{d}
$$

Here, $\Delta t$ is the lag time, $\Delta t_{d}$ is the traveltime difference between wave pulses travelling to stations $x$ and $y$, and $W$ is a windowing function describing the shape of the pulses. 
In Figure 2.1 we show the result of the cross-correlation function calculated according to equation 2.1 for different source-distributions. In this example, each source emits a Gaussian-shaped pulse with a maximum amplitude of one, a period of $T=5 \mathrm{~s}$, a duration of $2 T$, and the waves are recorded at two stations $300 \mathrm{~km}$ apart. The velocity at which the waves travel is $3 \mathrm{~km} / \mathrm{s}$.

From Figure 2.1, it can be clearly seen that integrating over sources spanning only a semicircle already results in a signal corresponding to a wave pulse travelling between stations $x$ and $y$ at a velocity of $3 \mathrm{~km} / \mathrm{s}$. However, only the causal part of the estimated Green's function can be retrieved and the cross-correlation contains spurious arrivals around zero lag-time. When integrating over the whole circle, we obtain two identical wave pulses corresponding to waves travelling at $3 \mathrm{~km} / \mathrm{s}$ from station $x$ to station $y$ at positive lag-times and from station $y$ to station $x$ at negative lag-times. Furthermore the spurious results at zero lag-time have disappeared. In other words, by integrating over sources entirely surrounding the stations, any arrivals corresponding to velocities faster than $3 \mathrm{~km} / \mathrm{s}$ interfere destructively, thereby illustrating the stationary-phase argument of Snieder [2004]. Consequently, integrating over sources within the range over which the cross-correlations interfere constructively, yields almost exactly the same wavefield as for the case of sources surrounding the two stations [see also Snieder and Wapenaar, 2010]. The small differences between the third and fourth panels in Figure 2.1 likely stem from approximating the integral in equation 2.1 by a sum.

While this example is an obvious over-simplification of any real situation and the crosscorrelation function is not the Green's function between the two stations, it is instructive in that it demonstrates how meaningful information from cross-correlation functions can be extracted even in the case of azimuthally biased noise source distributions, as theoretically shown by Wapenaar [2006] and Snieder [2004].

\subsection{Processing}

As discussed in the previous section, the reliability and accurateness of information extracted from noise cross-correlation functions depends on the spatial distribution of seismic noise sources. Shapiro and Campillo [2004] proposed that the spatial distribution of ambient seismic noise sources randomizes over time and that the estimated Green's function can therefore be computed from cross-correlating long durations of seismic noise recordings. The main challenge in obtaining such long continuous seismic noise records lies in the efficient suppression of earthquake signals and signals from stationary sources 

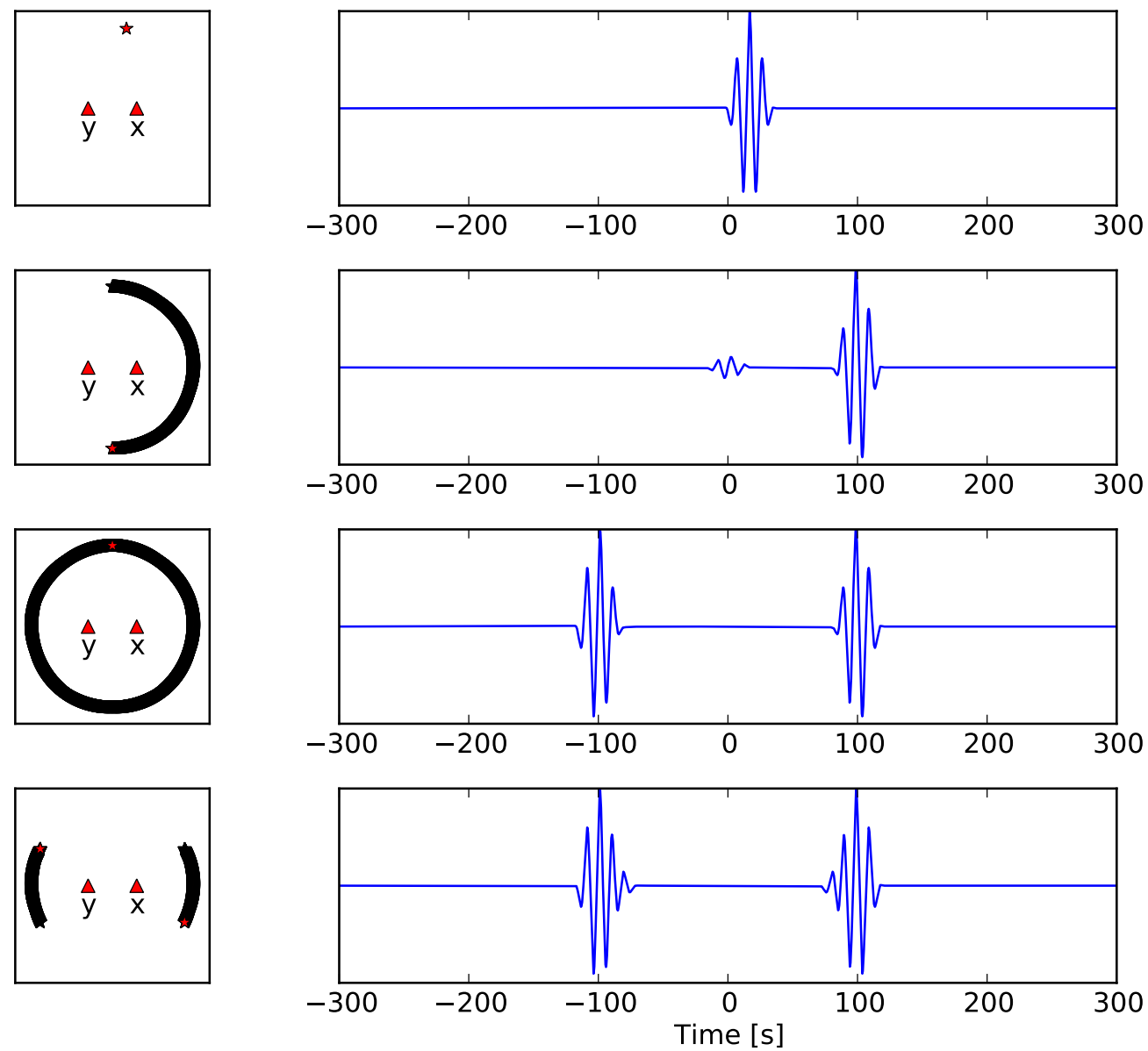

Figure 2.1 The left panels show the locations of noise sources (red stars) emitting Gaussian-shaped pulses recorded at stations $x$ and $y$ (red triangles) and then cross-correlated. The right panels show the sum of all cross-correlation functions calculated according to equation 2.1 for each source distribution in the corresponding left panel [after Yao and Van Der Hilst, 2009; Tsai, 2010]. 
[Bensen et al., 2007]. Different methods have been proposed to accomplish this: these include, for example, clipping amplitudes above a certain threshold [Sabra et al., 2005] or disregarding amplitude information completely by replacing positive amplitudes with 1 and negative amplitudes with -1 [Shapiro and Campillo, 2004; Shapiro et al., 2005].

In this study, we generally follow the processing scheme outlined by Bensen et al. [2007] which can be divided into four phases: pre-processing, cross-correlation, surface wave phase and group velocity measurements and quality control (Figure 2.2).

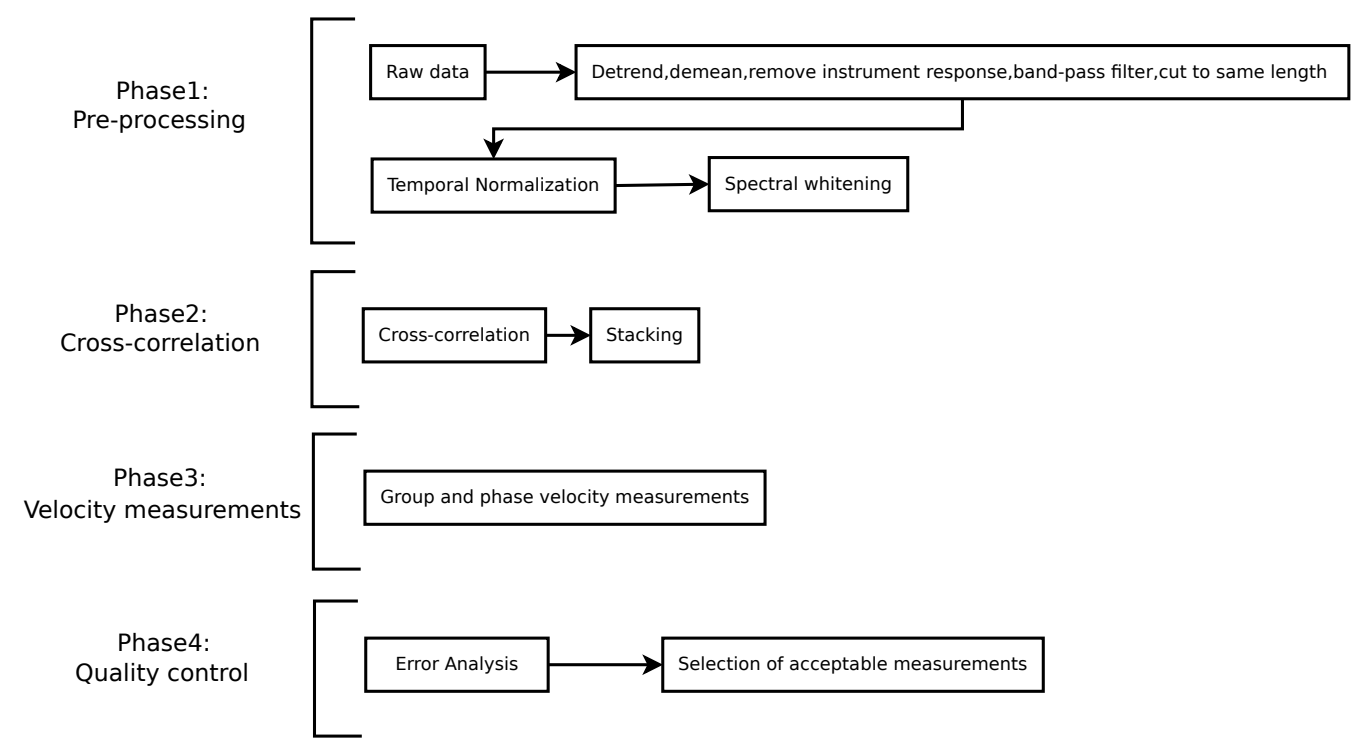

Figure 2.2 Simplified processing scheme used in this study after Bensen et al. [2007].

\subsubsection{Pre-processing}

In the first phase of the analysis, raw data from two stations spanning an identical time period are checked for gaps; the mean, trend and instrument response are removed; a broad band-pass filter is applied to stabilize the instrument response removal [Scherbaum, 2007]; and the records for each day are cut into 84000 s-long files. In order to reduce the effects of high-amplitude earthquake signals in the resulting cross-correlation functions, time domain normalisation is applied [Yang et al., 2007; Bensen et al., 2007]. This effectively downweights those parts of a seismogram containing earthquakes, by first bandpass filtering the trace within a period range typical for surface waves from earthquakes (15-50 s), and then inversely weighting the original trace with a smoothed version of the filtered trace. Finally, the influence of stationary, monochromatic noise sources is reduced by spectral whitening, that is by smoothing and broadening the seismogram's amplitude spectrum using a running average with a window length of $256 \mathrm{~s}$ [Lee, 1986]. Figure 2.3 
illustrates the difference in the amplitude spectra of a noise record before and after time domain normalization and spectral whitening were applied. The final spectrum is notably flatter than the original amplitude spectrum. All pre-processing steps only change the amplitude spectrum of a trace but not its phase spectrum.
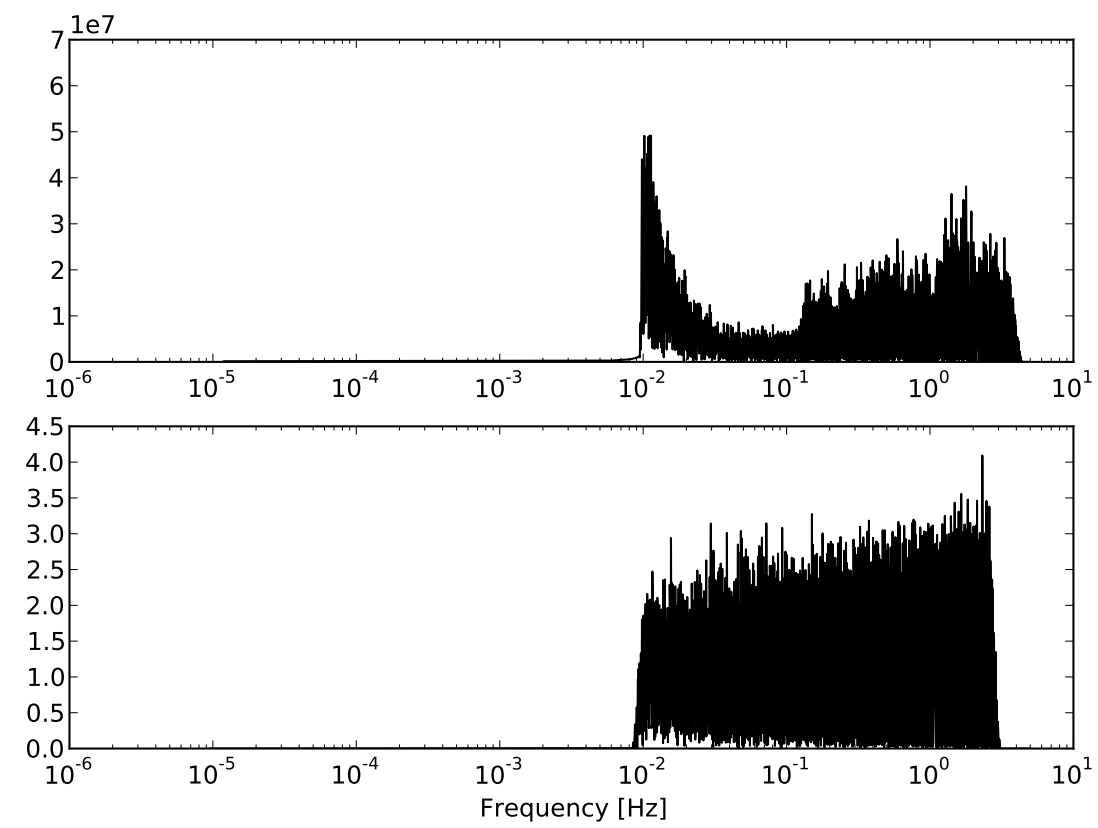

Figure 2.3 Amplitude spectrum for a vertical component seismogram recorded at GeoNet station CRLZ (Canterbury Ring Laser) before (top panel) and after (bottom panel) time domain normalization and spectral whitening are applied.

Following Lin et al. [2008] when processing horizontal components, we do not rotate the north and east components into the radial and transverse directions until after the crosscorrelations have been computed. This is possible since cross-correlation is a linear operation, and it has the advantage that fewer traces have to be rotated, which saves time and disk space. We apply the temporal normalization to the north and east components simultaneously by inversely weighting both traces by an average of the north and east component traces, band-pass filtered within $15-50$ s period. This ensures the consistency of the north and east components with the radial and transverse components. Using a conceptually identical method, Lin et al. [2008] demonstrated the commutivity of the rotation and all other processing steps involved in the pre-processing and the cross-correlation phases, using tests rotating the north and east component either at the beginning or at the end of the processing. 


\subsubsection{Cross-correlation and velocity measurements}

In the second phase of the analysis, daily cross-correlations are calculated between every two stations recording on the same day, and the results are then stacked over the available time span. In particular, we cross-correlate the vertical-vertical, east-east, north-north, north-east and east-north components. The cross-correlations of horizontal component seismograms are then rotated to obtain the cross-correlation functions for the radial and transverse components, as noted above. Although some other studies have included correlations between additional components, (notably vertical-radial correlations), in their analysis [e.g. Stehly et al., 2009], we focus here on the analysis of cross-correlations between identical components, that is the correlation functions between the verticalvertical, radial-radial and transverse-transverse components. We assume in this study that Rayleigh waves are elliptically polarized in the radial-vertical plane and that Love waves are linearly polarized in the transverse direction. Roueff et al. [2009] recently demonstrated a method of inferring the true polarization of Rayleigh and Love waves from noise cross-correlations but it has been shown in several studies that our assumption is an adequate approximation [Lin et al., 2008; Moschetti et al., 2010; Stehly et al., 2009].

In the third phase of the analysis we measure Rayleigh and Love wave phase and group velocities by employing an automatic multiple-filter technique [Dziewonski et al., 1969] also termed time-frequency analysis [FTAN; Levshin et al., 1972, 1992; Levshin and Ritzwoller, 2001]. To measure group velocities, the cross-correlation function is Fourier transformed and then filtered with a set of narrow-band Gaussian filters. For each filtered cross-correlation function, the maximum of its envelope is identified as the group travel time between the stations concerned. With $\omega$ being the angular frequency, the Gaussian filter $H\left(\omega_{o}\right)$ centered at angular frequency $\omega_{o}$ is defined as

$$
H\left(\omega_{o}\right)=e^{-\frac{\alpha}{\omega_{o}^{2}}\left(\omega-\omega_{o}\right)^{2}} .
$$

$\alpha$ is therefore the only parameter that controls FTAN and it is defined as follows:

$$
\alpha=\zeta 20 \sqrt{d / 1000}
$$

Here $d$ is the distance between the two stations in kilometres and $\zeta$ is a value given by the user. The resolution in frequency and time of FTAN depends on the width of the Gaussian filters. Wider filters improve resolution in time at the cost of resolution in frequency and vice versa. In other words, the greater $\zeta$ the better the frequency resolution. For a detailed discussion of the resolution of FTAN, see Feng and Teng [1983]. 
The discrete Fourier Transform of a finite timeseries causes spectral leakage, and as a consequence the central frequency of the Gaussian filter will not represent the frequency content of the filtered trace accurately [Press et al., 2005]. To obtain a more accurate measure of the angular frequency $\omega$ corresponding to a group velocity measurement, the instantaneous frequency is computed - that is, the frequency derived from the time derivative of the phase $\Phi$ of the filtered signal at the time corresponding to the maximum of the envelope $\left(\omega=|\partial \Phi / \partial t|_{t_{\max }}\right)$. The resulting group velocity curve is then used in a second step to create a phase-matched filter [e.g. Herrin and Goforth, 1977; Levshin and Ritzwoller, 2001]. This phase-matched filter is applied to the cross-correlation function to remove the dispersion, and, in doing that, compress the signal. Parts of the signal other than the surface wave train of interest will remain as side lobes of the compressed signal and can be filtered out by applying a Gaussian filter that is approximately the same width as the compressed signal. After re-dispersing the signal, phases other than the surface wave phases are largely attenuated. An example of the effect of phase-matched filtering on the amplitude map and group velocity resulting from FTAN is shown in Figure 2.4. Each column in the amplitude map represents the envelope amplitude of the noise cross-correlation function filtered at the corresponding period.

Lin et al. [2008] showed that the phase velocity $c$ at the time $t_{\max }$, corresponding to the group travel time, can be determined as

$$
c=\frac{\omega}{k}=\frac{r \omega}{\Phi\left(t_{\max }\right)+\omega t_{\max }-\frac{\pi}{4}-2 \pi N}
$$

Here $\omega$ is the angular frequency, $k$ the wave number, $r$ the distance between the two stations for which the cross-correlation was computed, $\Phi$ the phase of the filtered crosscorrelation function and $N$ the number of cycles the wave at frequency $\omega$ completed while travelling between the two stations. The factor $\pi / 4$ is caused by the influence of ambient noise sources lying off the great-circle path between the two stations [Harmon et al., 2007]. To determine the correct number of cycles $N$, prior knowledge of the earth's structure and the corresponding dispersion curves in the study area is needed. Differences in phase velocity due to different values of $N$ increase with increasing periods. Therefore the correct number of cycles is determined by comparing the measured dispersion curve with a reference dispersion curve at the longest period of interest, and then determining the number of cycles at successive shorter periods by requiring the dispersion curve to be smooth. We describe this process in more detail in Section 3.4.2. 

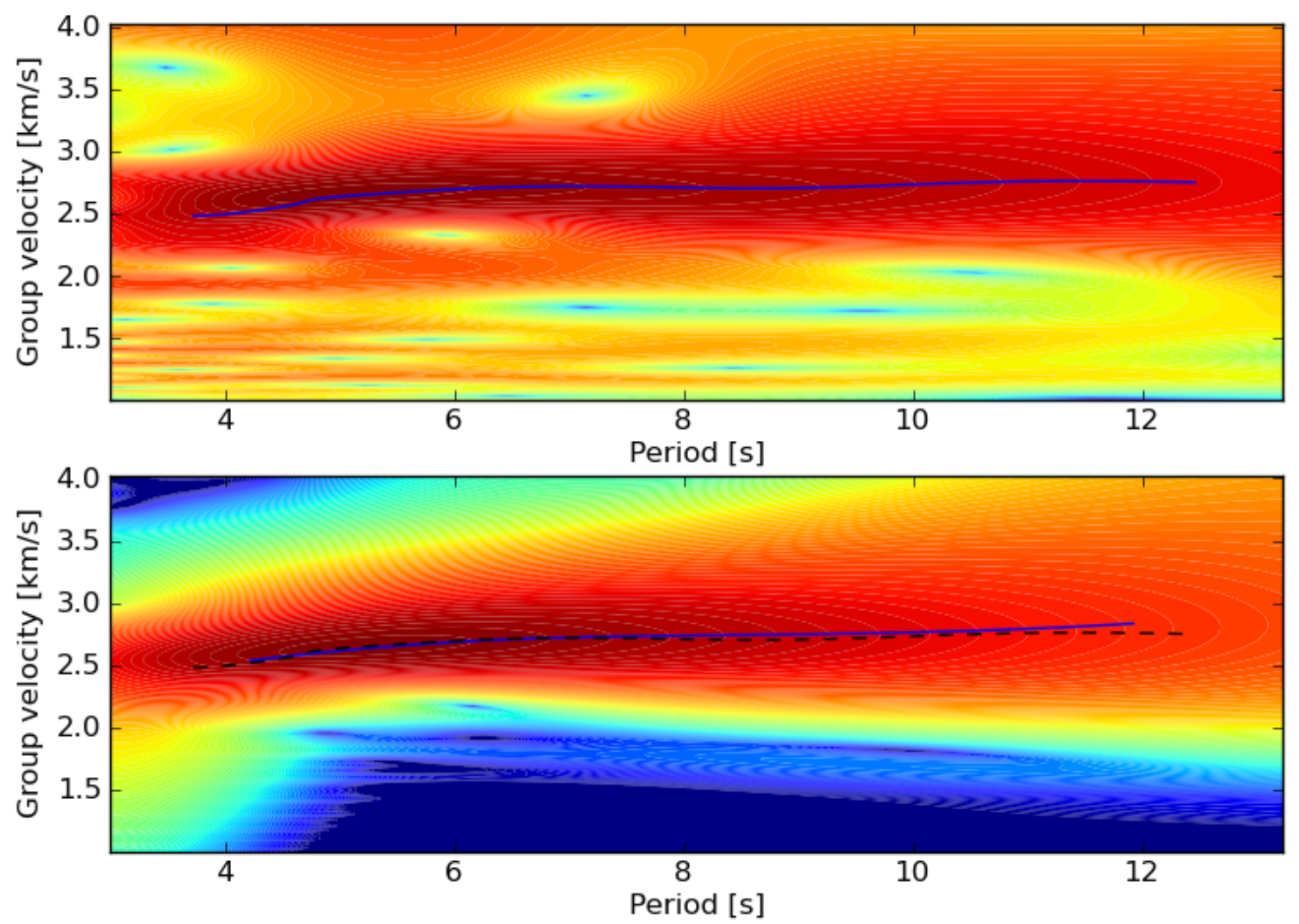

Figure 2.4 Example of the effect of employing FTAN with and without phase-matched filtering on the noise cross-correlation function between stations KARC (Karaitiana Farm) and S21 (no other name). The amplitude map and group velocity curve (blue line) on the top panel are computed without phase-matched filtering and the ones on the bottom panel with phase-matched filtering. The black dashed line on the bottom panel displays the group velocity dispersion curve from the top panel. 


\subsubsection{Quality control}

In the fourth phase of the analysis, selection criteria are applied to identify acceptable measurements. First, dispersion measurements at a particular period are rejected if the signal-to-noise ratio (SNR) of the corresponding cross-correlation function at this period lies below a certain threshold. The value of the threshold has to be chosen based on the data in order to retain only high-quality measurements while not reducing the number of measurement too severely (see Chapter 5).

Second, measurements between stations less than two wavelengths apart are rejected. This means for two stations $60 \mathrm{~km}$ apart and an assumed average velocity of $3 \mathrm{~km} / \mathrm{s}$ the maximum period for which velocity measurements are accepted is $T=60 /(2 \times 3) \mathrm{s}=10 \mathrm{~s}$. The required minimum number of wavelengths between two stations differs in previous studies and is imposed in order to avoid the causal and acausal portions of the cross-correlation functions interfering with each other. Bensen et al. [2007] suggested a minimum interstation distance of three wavelengths, while Shapiro et al. [2005] and Stehly et al. [2009] adopted a minimum distance of two wavelengths and Brenguier et al. [2007] used only one wavelength.

\subsection{Inversion}

As mentioned in Section 1, surface wave dispersion curves can be used in different ways to infer geological structures. In this study we invert dispersion curves for:

1. Surface wave velocity maps (Chapter 3);

2. Path averaged $1 \mathrm{D}$ shear velocity profiles (Chapters 4 and 6);

3. Pseudo-3D shear velocity models (Chapter 5);

In Section 2.4.1 we describe the algorithm used in this study to infer surface wave velocity maps at discrete periods from the ensemble of dispersion curves. Sections 2.4.2 and 2.4.3 address the topic of inverting single dispersion curves for path-averaged shear velocity profiles and a set of surface wave velocity maps at successive periods for pseudo-3D shear velocity models, respectively. 


\subsubsection{Surface wave tomography}

To determine the lateral distribution of surface wave velocities we employ the damped least squares method of Barmin et al. [2001], which minimizes the following penalty function:

$$
\chi(\mathbf{m})=(\mathbf{G m}-\mathbf{d})^{\dagger} \mathbf{C}^{-1}(\mathbf{G m}-\mathbf{d})+\mathbf{m}^{\dagger} \mathbf{Q m} .
$$

For a grid of $k$ cells and surface wave dispersion measurements between $n$ station pairs, $\mathbf{m}$ is the $k$-dimensional model vector of slowness perturbations from a $2 \mathrm{D}$ reference map at every grid point, $\mathbf{d}$ is the $n$-dimensional data vector of observed traveltime differences between station pairs compared to the theoretical travel time for the reference model; $\mathbf{Q}$ is a $k \times k$ regularization matrix; $\mathbf{C}$ is the $n \times n$ covariance matrix of observational errors; and $\dagger$ denotes the complex conjugate. The $n \times k$ matrix $\mathbf{G}$ is the forward model operator that links the model with the observations such that

$$
G_{i j}=\frac{d_{i j}}{c_{j}^{0}}
$$

Here $d_{i j}$ is the path length of the surface wave for station pair $i$ in cell $j$ and $c_{j}^{0}$ is the slowness value of the reference model in cell $j$. The regularization matrix $\mathbf{Q}$ can be decomposed as follows:

$$
\mathbf{Q}=\mathbf{F}^{\dagger} \mathbf{F}+\mathbf{H}^{\dagger} \mathbf{H}
$$

Here, $\mathbf{F}$ is a $k \times k$ matrix governing the spatial smoothness of the final model by including a spatial correlation length $\sigma$ as follows:

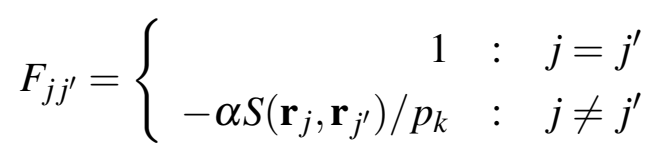

where $\mathbf{r}_{j}$ and $\mathbf{r}_{j^{\prime}}$ are the location vectors of cells $j$ and $j^{\prime}$,

$$
S\left(\mathbf{r}_{j}, \mathbf{r}_{j^{\prime}}\right)=\text { const. } \times \exp \left(-\frac{\left|\mathbf{r}_{j}-\mathbf{r}_{j^{\prime}}\right|^{2}}{2 \sigma^{2}}\right)
$$

and

$$
p_{k}=\sum_{j^{\prime}} S\left(\mathbf{r}_{j}, \mathbf{r}_{j^{\prime}}\right)
$$

Finally, $\mathbf{H}$ is a $k \times k$ matrix penalizing poor path coverage. For the path density $\rho\left(\mathbf{r}_{j}\right)$ in 
cell $j, \mathrm{H}$ is defined as

$$
H_{j, j^{\prime}}=\left\{\begin{array}{rll}
\beta \exp \left(-\rho\left(\mathbf{r}_{j}\right)\right) & : & j=j^{\prime} \\
0 & : & j \neq j^{\prime}
\end{array}\right.
$$

Solving $\partial \chi(\mathbf{m}) / \partial \mathbf{m}=0$ results in:

$$
\mathbf{m}=\left(\mathbf{G}^{\dagger} \mathbf{C}^{-1} \mathbf{G}+\mathbf{Q}\right)^{-1} \mathbf{G}^{\dagger} \mathbf{C}^{-1} \mathbf{d}
$$

The damping parameters $\alpha, \beta$ and $\sigma$ are defined a priori by the user. Tests show that our results are sensitive to $\alpha$ but mostly insensitive to $\beta$ and we could therefore simplify equation 2.7 to $\mathbf{Q}=\mathbf{F}^{\dagger} \mathbf{F}$. $\sigma$ needs to be larger than a minimum threshold for the model misfit distribution to be centered around zero. In Section 5.4 we discuss, in more detail, how we identify the best combination of damping parameters.

The resolution of this inversion method is explored by evaluating the change of a deltalike perturbation at each cell after applying the resolution matrix $\left(\left(\mathbf{R}=\mathbf{G}^{\dagger} \mathbf{C}^{-1} \mathbf{G}+\right.\right.$ $\left.\mathbf{Q})^{-1} \mathbf{G}^{\dagger} \mathbf{C}^{-1} \mathbf{G}\right)$. We return to the topic of the resolution of surface wave maps in Section 3.3.

\subsubsection{Inverting dispersion curves for shear velocity structure}

Inverting dispersion curves for shear velocity profiles is a highly nonlinear optimization problem [e.g. Dunkin, 1965]. One possible way to linearise this problem is to compute the partial derivatives of group $(\mathrm{U})$ and phase velocities $(\mathrm{C})$ versus shear velocity $(\partial \mathrm{U} / \partial \mathrm{Vs}$, $\partial \mathrm{C} / \partial \mathrm{Vs}$ ) with respect to depth for a starting model, and then employ an iterative gradient method to find the best-fitting model [e.g Harmon et al., 2007; Juliá et al., 2000; Horspool et al., 2006]. This approach suffers, however, from the obvious problem that it strongly depends on the starting model. It is further difficult to ascertain whether the bestfitting model is a global or local minimum of the optimization problem [Parker, 1994]. Another disadvantage of the linearised approach is that, unlike in true linear problems, the resolution matrix can only be approximated [Boschi, 2003].

To overcome some of these problems we have adopted the Neighbourhood Algorithm (NA), a quasi-random direct search method similar to genetic algorithms or simulated annealing [Sambridge, 1999a, b]. Sampling of the parameter space is steered by dividing it into Voronoi cells. Each Voronoi cell contains all points that are closer to its center than 
to any other point in the parameter space. Figure 2.5 shows an example of Voronoi cells in a two-dimensional parameter space, dividing the space between a random set of points.

The NA starts with a random number of points $\left(n_{s 0}\right)$ in the parameter space and assigns a misfit value to each point based on a user-defined forward model and a corresponding misfit function. In this study, the forward model calculates a synthetic dispersion curve for the parameters corresponding to a point in parameter space employing the algorithm proposed by Dunkin [1965]. The following misfit function $m$ is used to compute the difference between the observed and synthetic dispersion curves:

$$
m=\sqrt{\sum_{i}^{N} \frac{\left(x_{i}-x_{i, s y n}\right)^{2}}{\sigma_{i}^{2} N}} .
$$

Here $N$ is the number of periods at which surface wave velocity values are known, $x_{i}$ is the observed surface wave velocity and $x_{i, s y n}$ the synthetic surface wave velocity at period $i$, and $\sigma_{i}$ is the observational error at period $i$. Once the misfit has been calculated for every Voronoi cell, in a number of cells $\left(n_{r}\right)$ with the smallest misfit, a set of new points $\left(n_{s}\right)$ is produced randomly such that they follow an asymptotically uniform distribution in each cell. The Voronoi cells are then recomputed based on the old and new points and for each new point the misfit value is calculated. This process is repeated for a pre-defined number of iterations $\left(i t_{\max }\right)$. The four parameters $i t_{\max }, n_{s 0}, n_{r}$ and $n_{s}$ are defined by the user and together with the misfit function largely govern the way the parameter space is explored. Sambridge [2001] explored the role of these user defined parameters in finding acceptable models for a nonlinear inverse problem using the NA. He distinguished between the oversampled and the under-sampled case using the notion of 'saturation': with an increasing number of random points, the number of edges of Voronoi cells converges to a value that depends on the dimension of the parameter space (see Figure 2.6). Based on an empirical fit, the relation between the dimension $d$ of a parameter space and its saturation value $s$ is found to be

$$
s(d) \approx d^{3.5}
$$

For values below saturation, all Voronoi cells are neighbours of one another (undersampled) and for values above it there will be cells which don't share a common edge (oversampled). Consequently, in the oversampled case, it is more likely for the NA to get trapped in local minima than in the undersampled case. Conversely, in the undersampled case, the NA is more likely to suffer from a lack of concentration as it can jump into any other region of the parameter space at each iteration. Sambridge [2001] showed that in the oversampled case the NA should be tuned to be exploratory, that is large $n_{s}$ and $n_{r}$ should be chosen. In the undersampled case, he showed that the NA can be concentrated by the 
right choice of misfit function. Since, in this study, we use the NA implementation of Wathelet [2008], which does not allow a change of the misfit function, we ensure that our inversions converge by creating enough samples to oversample the parameter space.

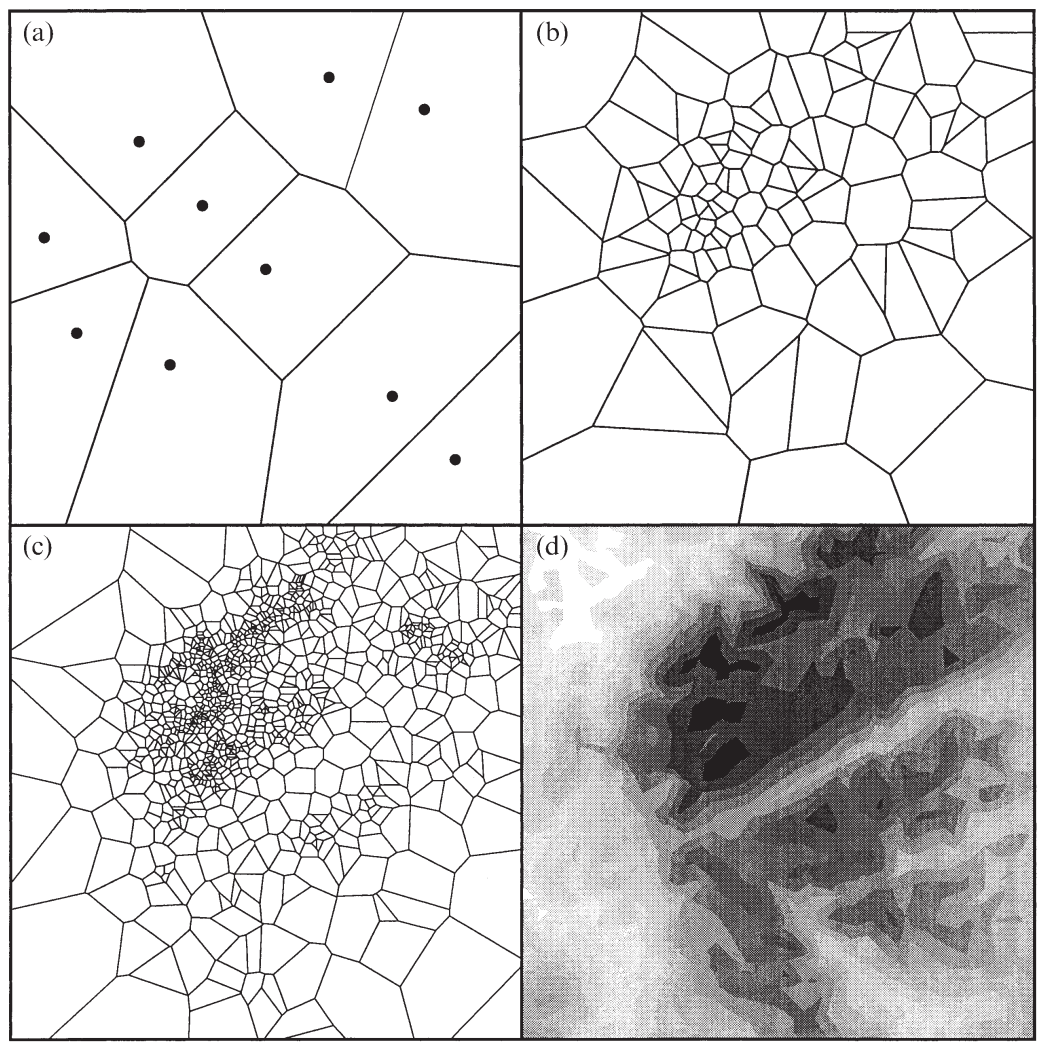

Figure 2.5 Voronoi cells in a two-dimensional parameter space for a) 10 , b) 100 and c) 1000 points created by the NA. d) shows the contours of misfits computed by a forward model routine. Misfits are smallest where the Voronoi cells are smallest and hence the density of points is highest [after Sambridge, 1999a].

\subsubsection{ID and pseudo-3D shear velocity modelling}

Dispersion curves, measured from the cross-correlation function between two seismic stations, can be inverted for an ensemble of path-averaged shear velocity profiles using the NA. Alternatively, a group of dispersion curves can be inverted for pseudo-3D shear velocity structure. This is done in this study in three steps: first, the study area is parameterised using a regular grid and phase and group velocities at successive discrete periods are inverted for maps of lateral velocity distribution using the algorithm described in Section 2.4.1; second, at each grid point values from the surface wave maps at successive periods are interpolated to form a local dispersion curve. For example, surface wave maps at 10 successive periods between 5 and $20 \mathrm{~s}$, discretized by a $20 \times 20$ cell grid, results in 400 dispersion curves each spanning the period range of 5 and 20 s period; third, 


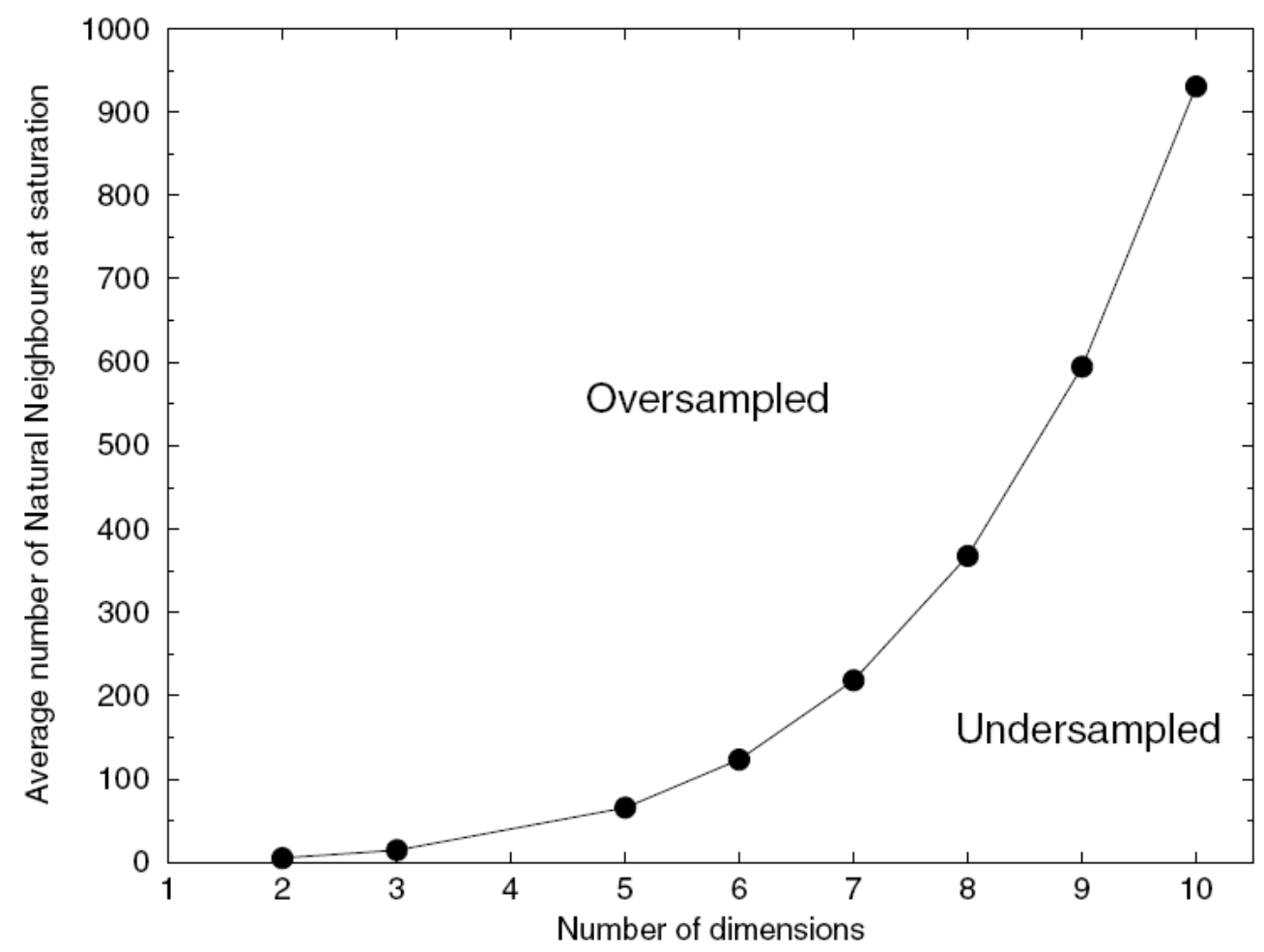

Figure 2.6 Dependence of the number of natural neighbours on the dimensionality of the parameter space [from Sambridge, 2001].

the dispersion curve at each grid point is inverted using the NA to obtain an ensemble of 1D shear velocity models. The average models at each grid point are then amalgamated, using a linear interpolation, to form a pseudo-3D volume.

We make further use of the whole ensemble of models to highlight the range of shear velocity profiles compatible with particular dispersion curves (Chapter 4 and 6) and to compute the uncertainties of model parameters (Chapter 4 and 5).

As we discuss in more detail in Section 5.22, this is only one of several possibilities to invert surface wave information for 3D or pseudo-3D structure. However we believe that it is a good compromise between more sophisticated inversion methods, like full waveform inversion [Tape et al., 2010, e.g.], and more simplified schemes, like iterative gradient methods, in that we account for the non-linearity of the problem but keep the computational costs as small as possible. However, this comes at the cost of having to invert twice, first for surface wave maps and then for 1D shear velocity profiles, with each inversion result being afflicted by ambiguities and uncertainties. Combined with the non-linear relationship between shear velocity models and dispersion curves, it makes the estimation 
of the effect of uncertainties in the cross-correlation functions on the final pseudo-3D model difficult. Consequently, our error analysis in Chapter 5 is purely empirical. As we describe in Chapter 6, estimating deviations of the cross-correlation functions from the true Green's function is also not trivial and requires knowledge of the noise source distributions.

Like most other geophysical optimization problems, inverting surface wave velocities for shear velocity structure is therefore not a well constrained problem, and we interpret the results as carefully as possible. 
2. Methodology 


\section{Rayleigh and Love wave phase velocity maps of New Zealand}

Surface wave tomography — inversion of surface wave phase or group velocity dispersion curves for their lateral distribution — has been traditionally used in earthquake seismology [e.g. Mocquet et al., 1989; Ritzwoller and Levshin, 1998; Shapiro et al., 2004, and references therein]. We demonstrate in this chapter the inversion of surface wave dispersion curves from ambient seismic noise cross-correlations for surface wave maps of Rayleigh group and phase and Love wave phase speeds in New Zealand at discrete periods between 5 and $23 \mathrm{~s}$. This eliminates the dependence on the source location inherent to surface waves from earthquakes and complements surface wave measurements from teleseismic events by making surface wave speed measurements at short periods $(\sim 5-$ $25 \mathrm{~s}$ ). The results for Rayleigh and Love wave velocity maps are compatible with known geological and tectonic formations in New Zealand. Due to differences in their respective sensitivity kernels, Rayleigh wave and Love wave phase velocity maps as well as Rayleigh wave phase and group velocity maps exhibit notable differences at identical periods. These differences in sensitivity with respect to depth are valuable when inferring shear velocity structure from surface wave velocity maps, as they complement each other at identical periods (see Chapter 5).

\subsection{Introduction}

In the first ambient noise tomography study conducted in New Zealand, Lin et al. [2007] measured Rayleigh wave group velocities across the country from noise correlations between 42 permanent seismic stations. In this chapter we extend that analysis by including data from several temporary deployments in our dataset. This increases the path coverage, most notably in the central North Island and the southern South Island. We start by measuring Rayleigh wave group velocities from noise correlations between stations in the 
expanded dataset and construct surface wave velocity maps, to ensure that they are consistent with the results of Lin et al. [2007]. We then extend this analysis to Rayleigh and Love wave phase velocities. For the inversion, we employ a damped least-squares method, implemented by Barmin et al. [2001], described in more detail in Section 2.4.1.

The additional difficulty inherent in measuring phase velocities rather than group velocities stems from the need to determine the number of complete cycles (i.e. integer wavelengths) between any two stations (see Section 2.3.2) and to identify and correct for $180^{\circ}$ phase shifts due to wrong wiring of seismometers (see Section 5.3.1). These problems and their solutions are discussed in more detail in Section 3.4.2. Fewer good quality dispersion curves are obtained for Love waves than for Rayleigh waves due to the lower signal-to-noise ratio (SNR) for Love waves but also because energy from Rayleigh waves ends up on the transverse component due to multipathing [Goforth and Herrin, 1979]. As discussed in more detail in Section 5.4, the lower SNR values are probably caused by the different excitation mechanisms for Rayleigh and Love waves.

\subsection{Datasets}

We determine Rayleigh and Love wave phase and group velocities at periods between $5 \mathrm{~s}$ and $25 \mathrm{~s}$ from ambient seismic noise recorded at stations of the following deployments (Figure 3.1):

- Southern Alps Passive Seismic Experiment [30 stations; 14 months; Anderson et al., 1997];

- Northland Deployment [NORD; 5 stations; 8 months; Duclos, 2005];

- Marlborough Deployment [7 stations; 6 months; Wilson et al., 2004];

- Central North Island Passive Seismic Experiment [CNIPSE; 33 stations; 6 months; Reyners and Stuart, 2002];

- Western Central North Island Passive Seismic Experiment [WCNIPSE; 6 stations; 9-11 months; Greve et al., 2008];

- Seismic Tomography Around Ruapehu and Tongariro deployment [START; 28 stations; 5 months; Rowlands et al., 2005];

- RF2004 deployment [7 stations; 10 months; Bannister et al., 2004];

- Permanent GeoNet stations (9-44 stations, 1-7 years). 
Although the deployments were not all concurrent (see Figure 3.2), including them significantly increases the path coverage in the central North Island and the central and southern South Island. At periods shorter than $5 \mathrm{~s}$, usable surface wave velocity measurements remain sparse due to a decrease in SNR values of the cross-correlations and above $25 \mathrm{~s}$ period, paths longer than two wavelengths (see Section 2.3.3) tend to be aligned in an approximately north-south direction and therefore provide insufficient crossing rays for a reliable dispersion measurement inversion.

\subsection{Resolution}

We consider the question of the surface wave maps' lateral resolution by first employing a checkerboard test with $1^{\circ} \times 1^{\circ}$ cells varying between $2.4,2.6,2.8$ and $3.0 \mathrm{~km} / \mathrm{s}$ (Figure 3.3). By tracing rays through the checkerboard model between the stations represented in our dataset, we obtain a synthetic dataset which we can invert in the same way that we would invert measured dispersion values at each of a number of discrete periods. Results for the $8 \mathrm{~s}, 13 \mathrm{~s}, 18 \mathrm{~s}$ and $23 \mathrm{~s}$ periods are shown in Figure 3.4. Cells in the central North Island and in the central and southern South Island are resolved best, in the sense that the original checkerboard pattern is retrieved, but all cells and especially those lying between the northern South Island and the southern North Island experience north-south smearing. This is most likely due to most interstation paths in this region being oriented either northeast-southwest or northwest-southeast, reducing the azimuthal range of paths in this region. At longer periods, the number of paths subparallel to the overall strike of New Zealand increases relative to that of paths in the orthogonal direction as a consequence of the requirement of a minimum two-wavelength interstation distance (Section 2.3.3). Accordingly, the north-south smearing of structures becomes more severe at longer periods. A map of azimuthal coverage (Figure 3.5) confirms this, showing high values of azimuthal coverage in those areas where the input checkerboard model is best resolved.

Another feature of the checkerboard test apparent in Figure 3.4 is the damping of velocity values towards the average of the velocity map. This is a result of the inversion algorithm employed (Section 2.4.1), which requires the inversion result to be smooth. Sharp velocity boundaries in the input model will therefore be broadened and lower in amplitude in the inversion results.

The second method we use here to assess resolution involves applying the resolution matrix to a delta-shaped perturbation at each node of the spatial grid used in the inversion [Barmin et al., 2001]. This yields the amount of blurring that is inherent to the inversion 


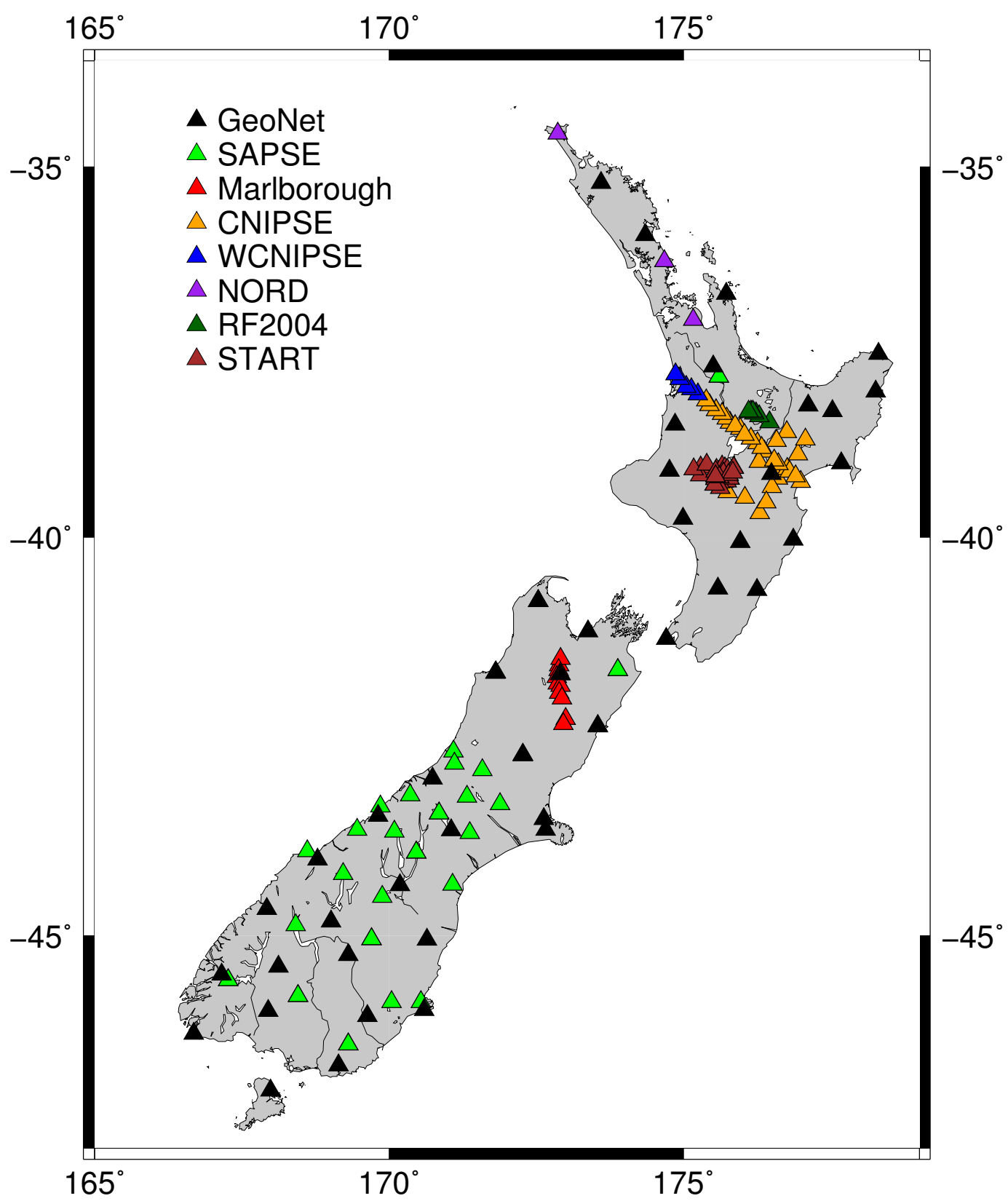

Figure 3.1 Seismic broadband stations used for ambient noise tomography. 


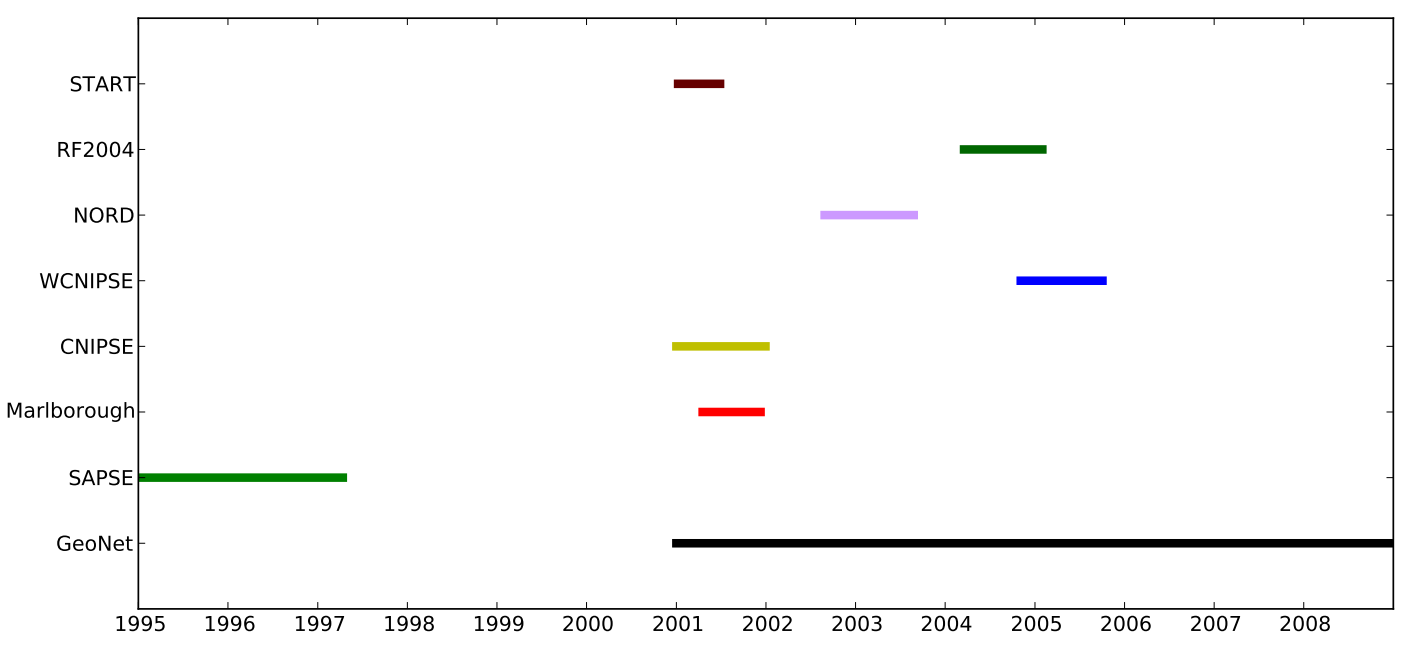

Figure 3.2 Timespans over which stations of the deployments shown in Figure 3.1 were operating.

process at every spatial node, and its principal advantage over the checkerboard test is that it corresponds to a resolution value at each node that can be evaluated quantitatively. This is done by fitting a cone to the resolution map at each spatial node with the resolution then defined as the radius of this cone. The results from the checkerboard tests show that resolution is likely to be direction-dependent with higher resolution in the east-west direction than in the north-south direction. This could be accounted for by fitting an elliptical cone instead of a circular cone to the resolution maps. It is beyond the scope of this study but is an obvious avenue for future work on nationwide surface wave maps.

Figure 3.6 shows that, in general, areas of high resolution on the North Island, inferred from the resolution matrix, are consistent with the results of the checkerboard test (Figure 3.4). However, low resolution in the southern South Island and high resolution in the region between the northern South Island and the southern North Island appears to be inconsistent with the results of the checkerboard test. Lévêque et al. [1993] showed that if an arbitrary synthetic model, such as that used in a checkerboard test, does not sample the space of possible models well enough, the results of such a test can be misleading. In particular, parts of the input model which lie within the null-space of the resolution matrix appear poorly resolved whereas a different input model without any components in the null space might show good resolution for the same parts of the model. This can even lead to the counterintuitive result that small-scale features are better resolved than large-scale features. Nolet et al. [1999] further pointed out that it is inefficient to infer the resolution of a single cell from checkerboard tests, as the same test needs to be run for every cell. Hence, while checkerboard tests can be instructive, they clearly need to be 


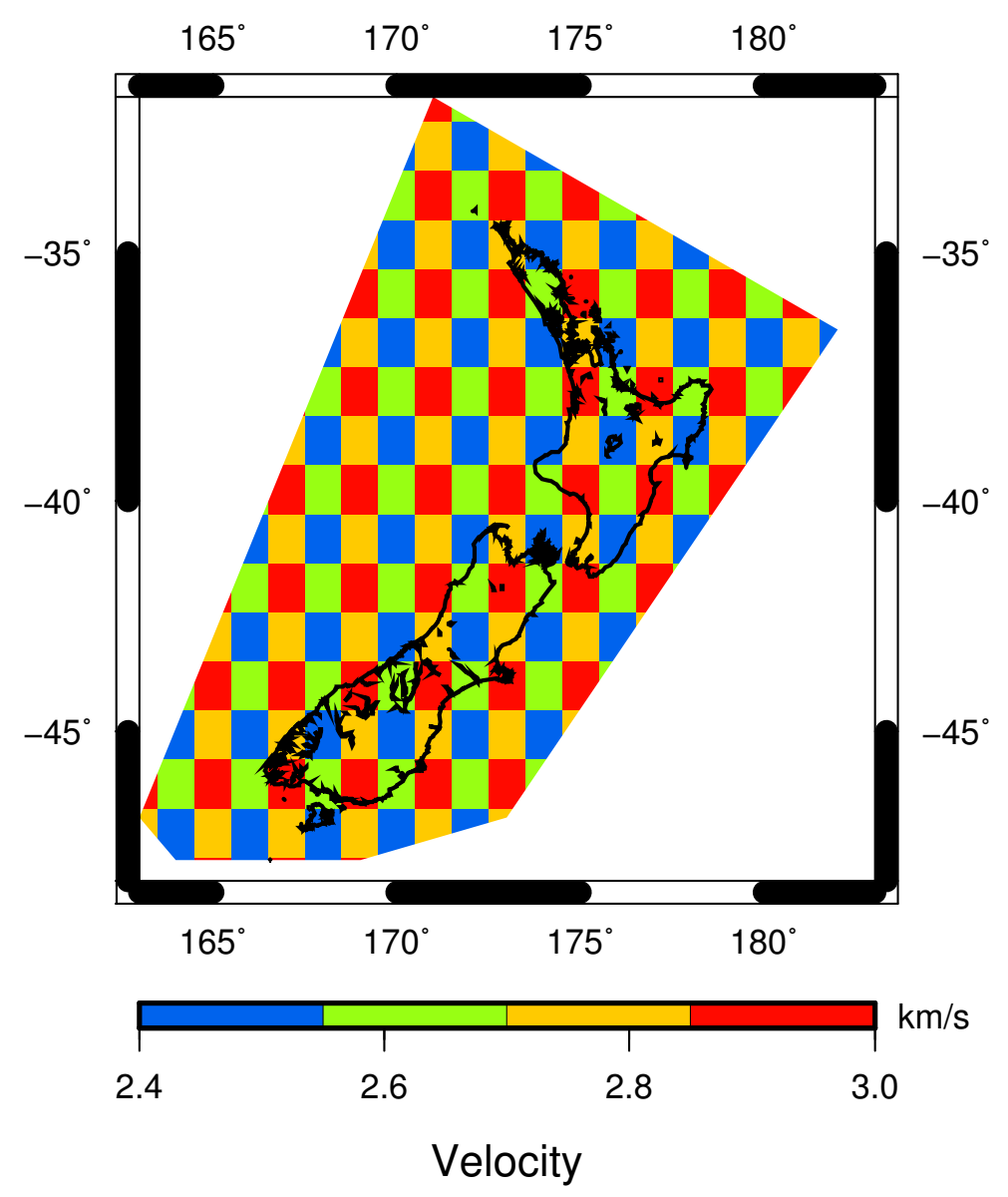

Figure 3.3 Checkerboard test input model. Every colour corresponds to the annotated velocity, only, despite the colour bar being continuous.

interpreted in conjunction with different methods which explore the full space of possible models, such as the one proposed by Barmin et al. [2001].

The Backus-Gilbert method [Backus and Gilbert, 1967, 1968] is a more quantitative way of assessing resolution than the two previously described methods. Its aim is to construct an averaging kernel for every point in a model from the components of the linear forward model operator (Section 2.4.1). Parker [1994] pointed out that for the realistic case of noisy data the Backus-Gilbert method is difficult to implement and does not necessarily yield more information than a method which explores the characteristics of the resolution matrix. Hence, we have chosen in this study to use two complementary methods of describing resolution. 

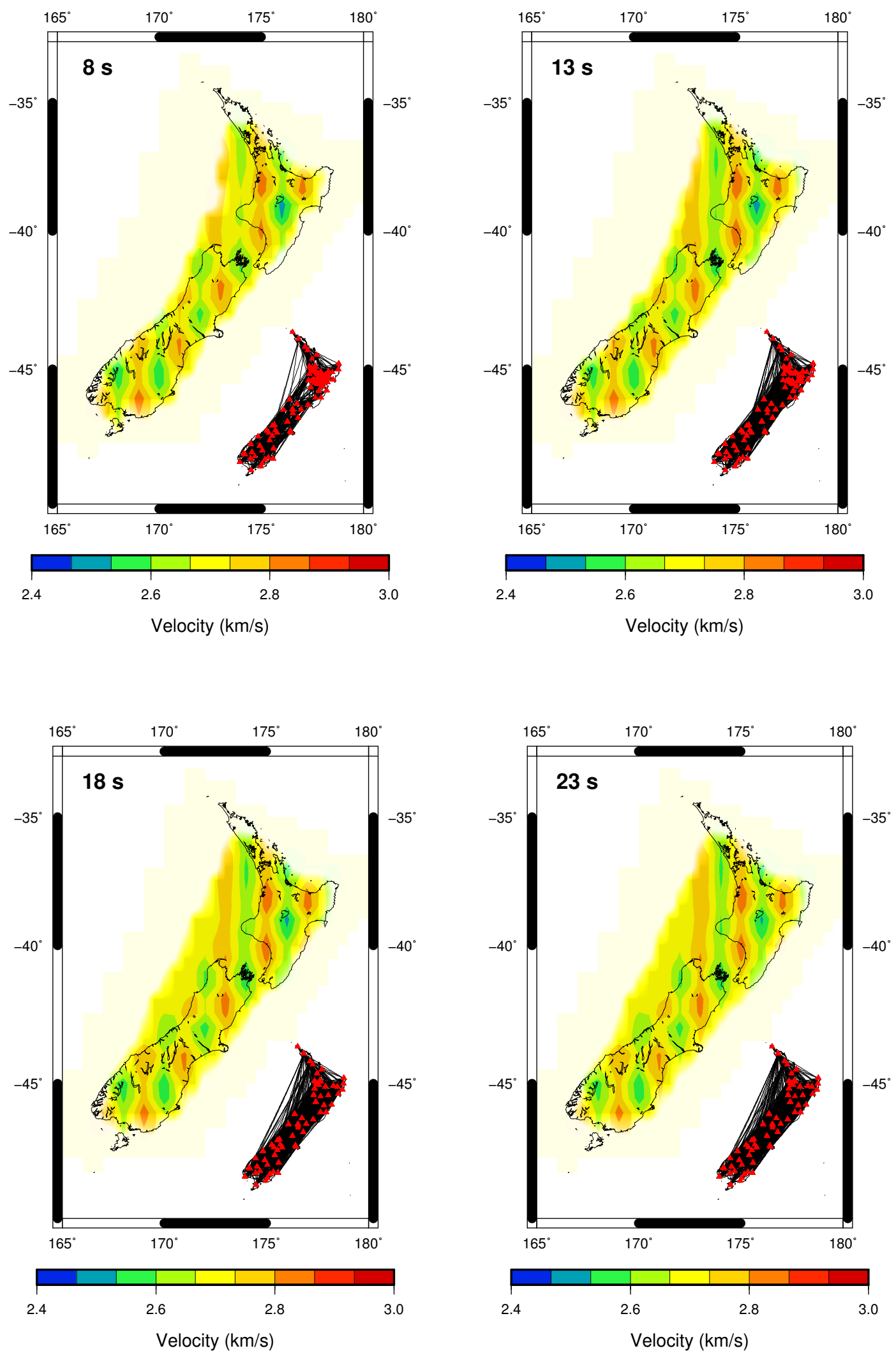

Figure 3.4 Results of the checkerboard test using the input model from Figure 3.3 for path distributions of Rayleigh wave group velocities at the $8 \mathrm{~s}, 13 \mathrm{~s}, 18 \mathrm{~s}$ and $23 \mathrm{~s}$ period. The inset in the lower right corner shows the path distribution of Rayleigh wave group velocity measurements for the corresponding period and the red triangles mark the seismic station locations. 

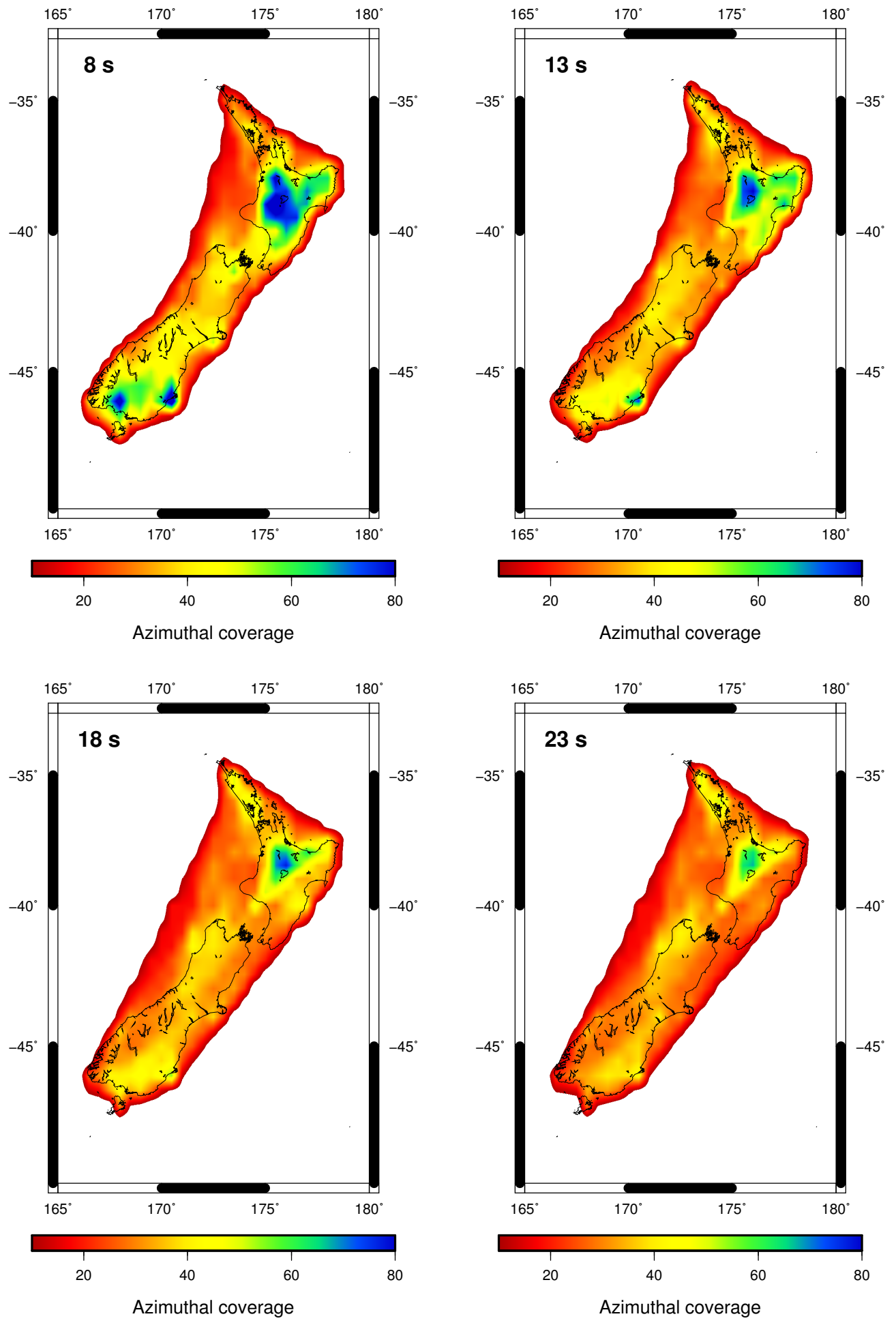

Figure 3.5 Azimuthal path coverage for each cell of the grid used in the inversion and the path distributions of Rayleigh wave group velocities at the $8 \mathrm{~s}, 13 \mathrm{~s}, 18 \mathrm{~s}$ and $23 \mathrm{~s}$ period. A value of 180 would correspond to complete azimuthal coverage. 

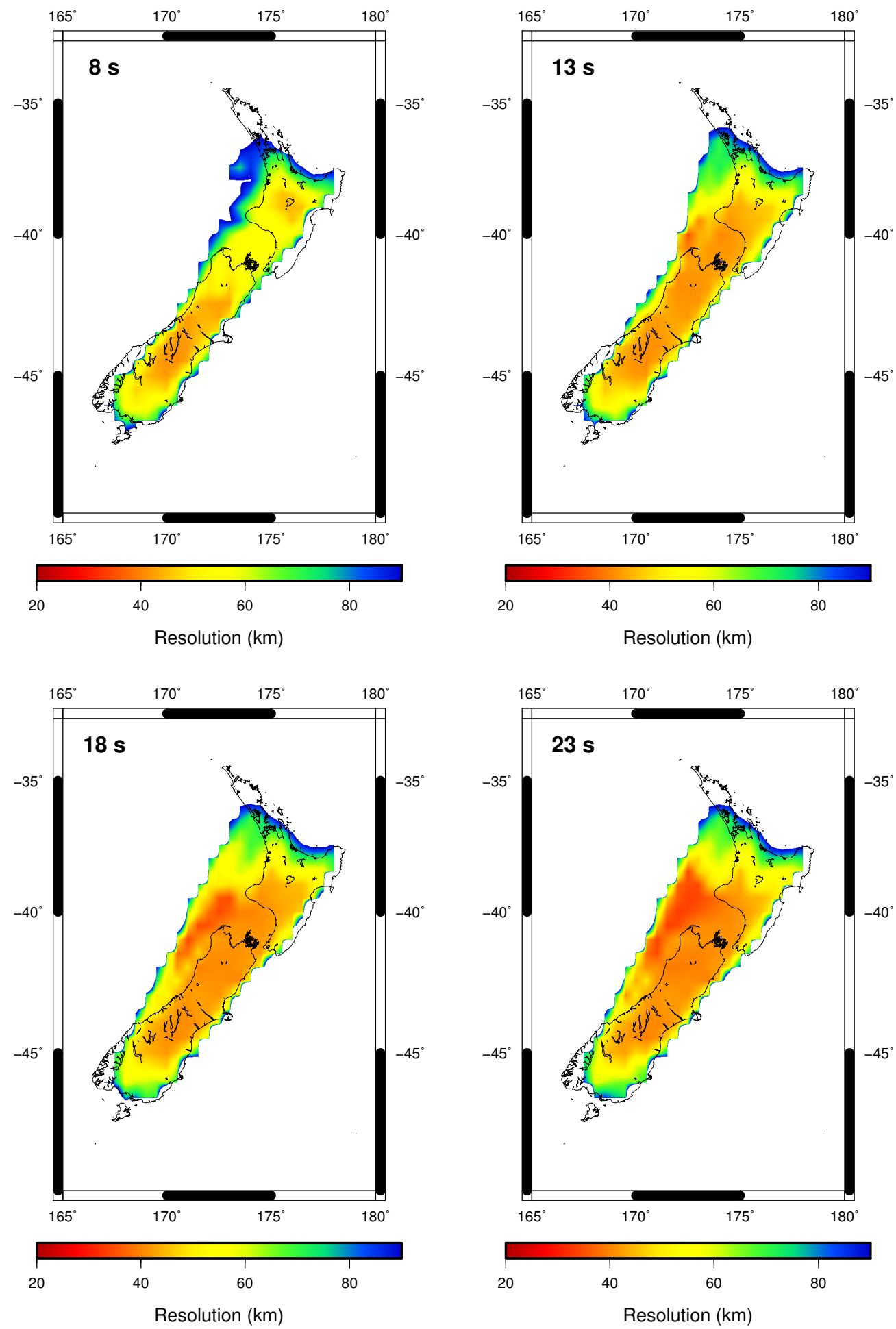

Figure 3.6 Resolution analysis for the path distributions of Rayleigh wave group velocities at the $8 \mathrm{~s}, 13 \mathrm{~s}$, $18 \mathrm{~s}$ and $23 \mathrm{~s}$ period following Barmin et al. [2001]. The resolution is defined as the distance at which two delta-shaped features can be distinguished unambiguously. 


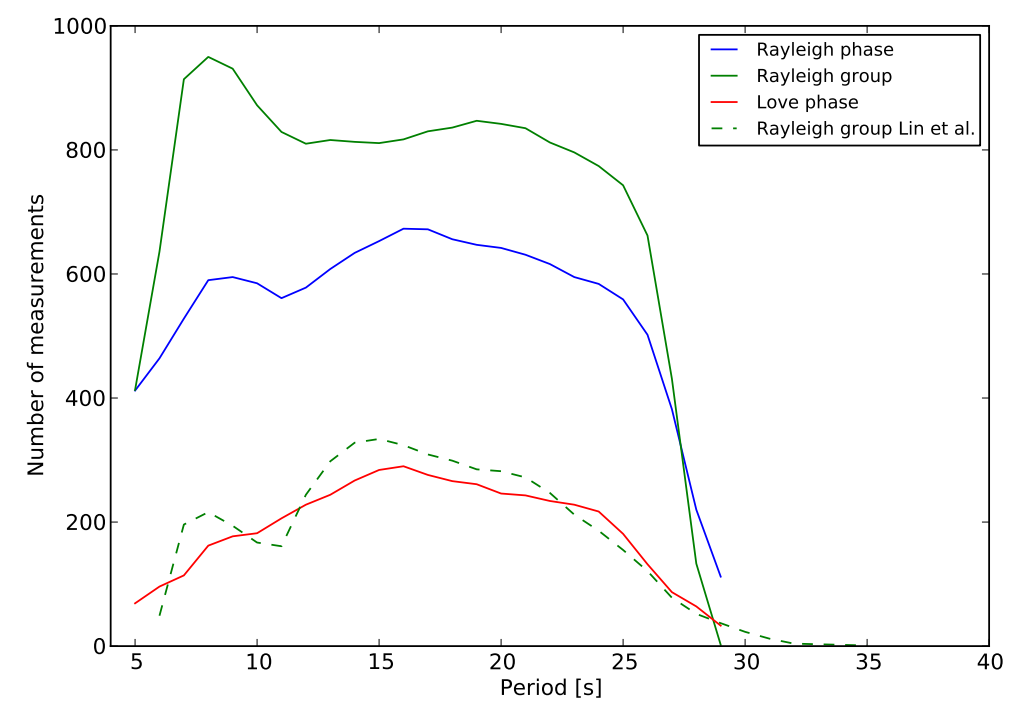

Figure 3.7 Number of velocity measurements versus period for Rayleigh and Love wave phase velocities.

\subsection{Results}

\subsubsection{Rayleigh wave group velocities}

The correlation of vertical-vertical component ambient noise recordings yields more than 3300 correlation functions. For more than 2400 of these, Rayleigh group velocity dispersion curves can be measured. The quality criteria discussed in Section 2.3.3 are approximately met by one third of the group velocity measurements at $8 \mathrm{~s}$ period, a figure nearly three times larger than Lin et al. [2007] obtained using only permanent seismic stations (Figure 3.7). Lin et al. [2007] employed seasonal stability of the cross-correlations as a further quality criterion: that is, correlation functions stacked over, for example, the spring months were required to be similar to correlation functions stacked over the winter months. The short deployment times of many of the temporary stations considered here makes the evaluation of seasonal variations of the cross-correlations difficult and we therefore did not apply this selection criterion which makes the comparison of the number of paths somewhat indirect. As our results are highly consistent overall with those of Lin et al. [2007] (shown in Figure 3.8), we will in the following only discuss differences between the Rayleigh wave group velocity maps of the two studies.

We show the surface wave maps for Rayleigh wave group velocities in Figure 3.9 at the same discrete periods illustrated by Lin et al. [2007]. At 8 s period, we observe velocities of $\sim 3.1 \mathrm{~km} / \mathrm{s}$ at the southern end of the South Island which is higher than the $\sim 2.8 \mathrm{~km} / \mathrm{s}$ 
inferred by Lin et al. [2007] for the same region. Basement rocks in this area are mostly greywacke and plutonic rocks which most likely account for the fast velocities. Stations from the SAPSE deployment as well as one new station on Stewart Island (Figure 3.10) improve the path coverage in this area significantly, which explains the differences to the results of Lin et al. [2007]. The 13 s period map (Figure 3.9) shows the same somewhat higher velocities relative to those of Lin et al. [2007] in the southern South Island, and more pronounced slow velocities of $\sim 2.2 \mathrm{~km} / \mathrm{s}$ within the Canterbury Basin (Figure $3.10)$.

The most remarkable differences at the $18 \mathrm{~s}$ period are the broader extension of slow velocities $(\sim 2.6 \mathrm{~km} / \mathrm{s})$ in the Taranaki Basin and the fast velocities of $2.9-3.1 \mathrm{~km} / \mathrm{s}$ to the west of New Zealand and in the southern South Island as opposed to $2.7-2.8 \mathrm{~km} / \mathrm{s}$ in the results of Lin et al. [2007]. The Taranaki Basin contains sediment deposits of up to $9 \mathrm{~km}$ thickness [King and Thrasher, 1996; Baur et al., 2010] and therefore is likely to be present as a region of slow velocities even at such long periods. Paths between stations to the west, offshore of New Zealand, are mainly northwest-southeast striking and we therefore cannot determine whether the linear structure of the zone of fast velocities in this area corresponds to the extent of the geological structure or whether it is an artifact of the path distribution. Rayleigh waves at this period are likely to image the fast velocities of the lower crust as can be seen from the corresponding sensitivity kernel (Figure 3.9).

The 23 s period maps of Lin et al. [2007] and of this study exhibit similar differences as the $18 \mathrm{~s}$ maps in the offshore region to the west of New Zealand and in the southern South Island with velocities in our study being approximately $0.4 \mathrm{~km} / \mathrm{s}$ faster than described by Lin et al. [2007]. Velocities on the North Island show similar patterns overall but velocities on the Northland Peninsula observed in this study are $\sim 0.3 \mathrm{~km} / \mathrm{s}$ faster and velocities in the central North Island exhibit more detail. For example, our results reveal slow velocities of $\sim 2.7 \mathrm{~km} / \mathrm{s}$ in the Taupo Volcanic Zone whereas the results of Lin et al. [2007] show the average velocity for the $23 \mathrm{~s}$ period map of $\sim 2.85 \mathrm{~km} / \mathrm{s}$. Slow velocities $(\sim 2.6 \mathrm{~km} / \mathrm{s})$ on the East Coast of the North Island, which are likewise influenced by the subducting Pacific slab and the accretionary prism, terminate further north in this study than displayed in the $23 \mathrm{~s}$ map of Lin et al. [2007].

\subsubsection{Rayleigh and Love wave phase velocities}

As mentioned above, the unknown number of cycles a wave has experienced while travelling between two stations - "integer ambiguity" — and possible $180^{\circ}$ phase shifts due to 

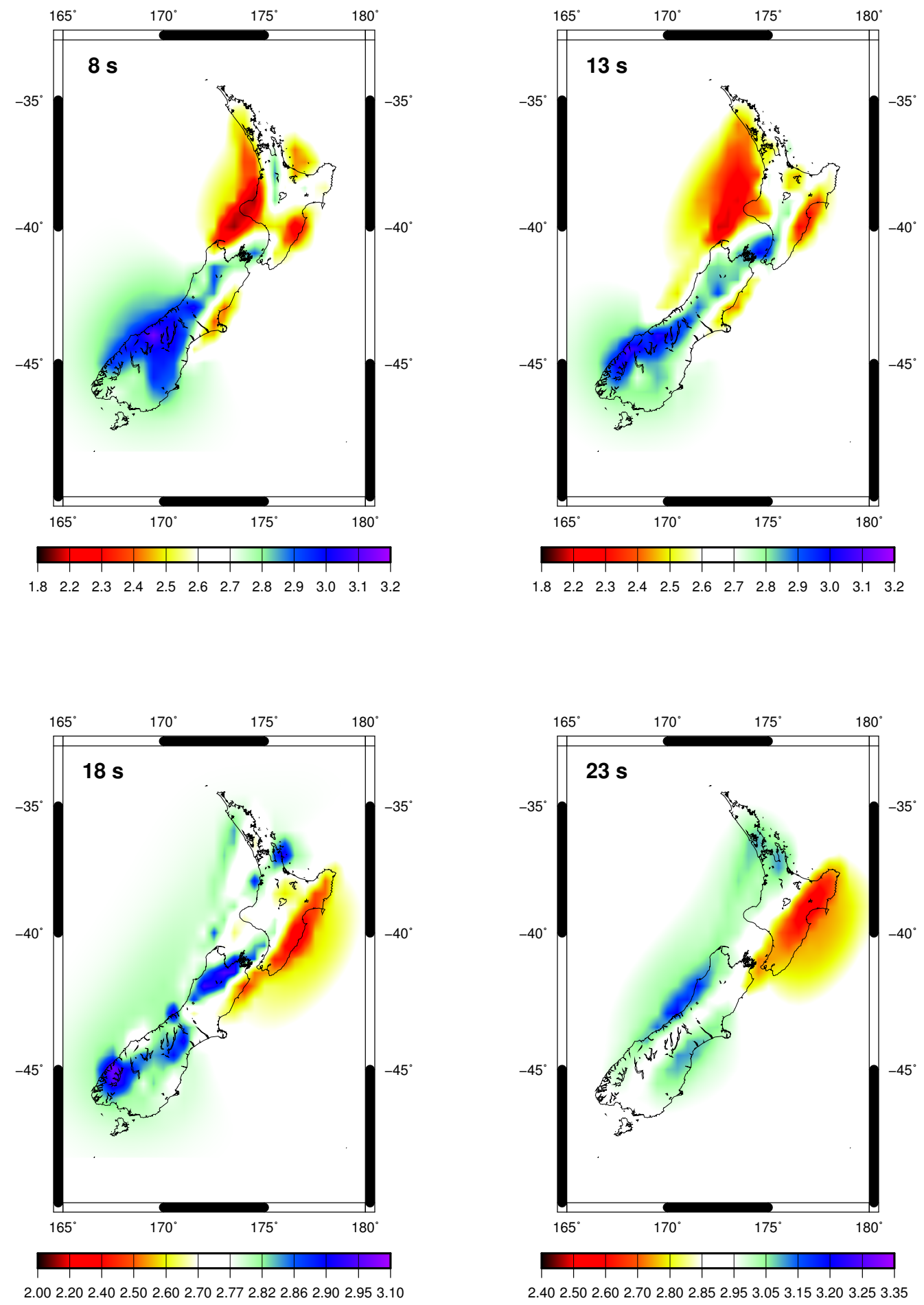

Figure 3.8 Rayleigh wave group velocity maps at the $8 \mathrm{~s}, 13 \mathrm{~s}, 18 \mathrm{~s}$ and $23 \mathrm{~s}$ period from the study of Lin et al. [2007]. Fan-Chi Lin kindly provided us with the data and colour palette required to reproduce Figure 11 of Lin et al. [2007]. 

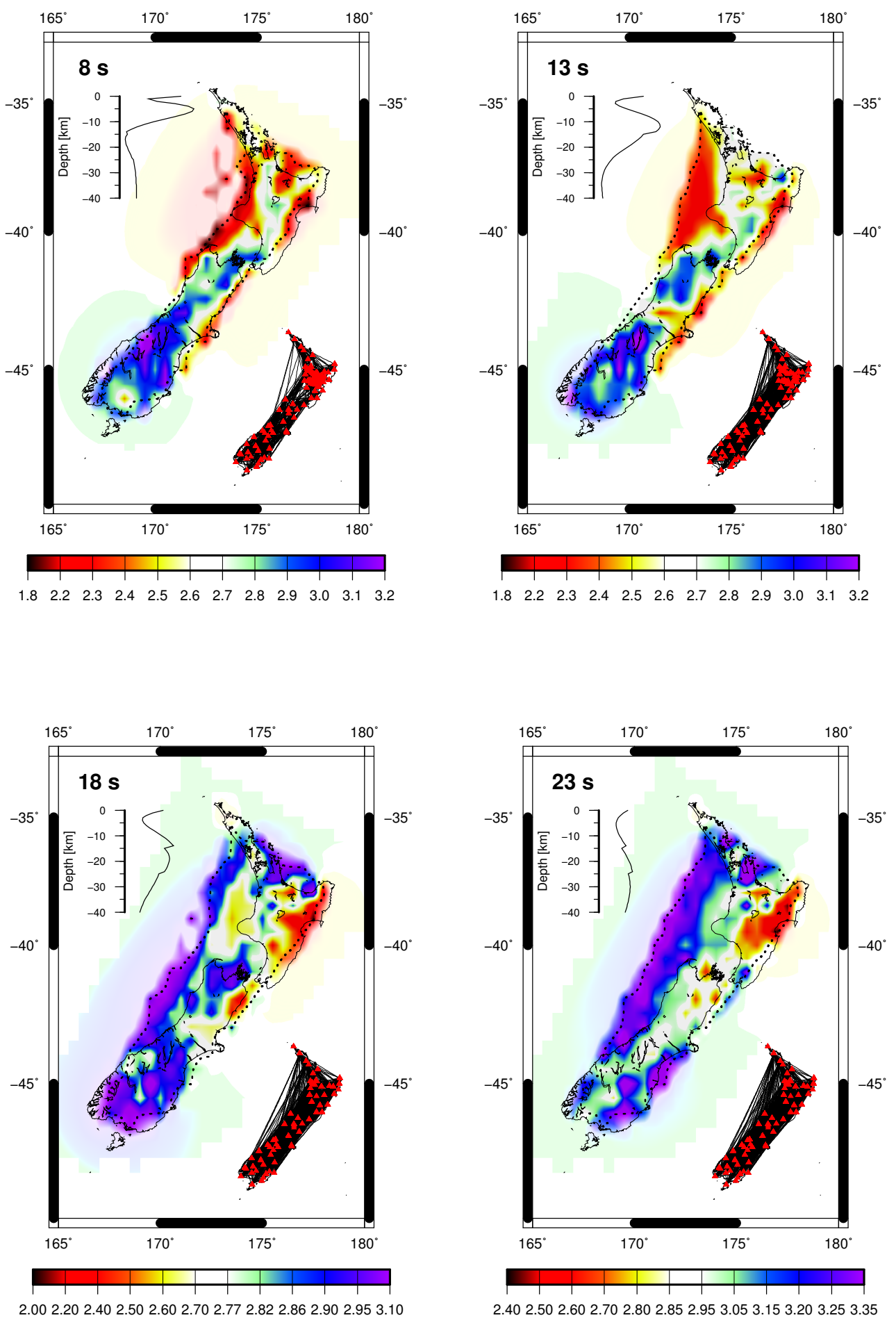

Figure 3.9 Rayleigh wave group velocity maps at the $8 \mathrm{~s}, 13 \mathrm{~s}, 18 \mathrm{~s}$ and $23 \mathrm{~s}$ period with the dashed line constraining the area within which resolution is better than $100 \mathrm{~km}$. Colours outside this area are toned down. The inset in the upper left corner shows the sensitivity kernel of group velocity to changes in S-velocity $(\partial \mathrm{U} / \partial \mathrm{Vs})$ with respect to depth at the corresponding period calculated for PREM [Dziewonski and Anderson, 1981]. The inset in the lower right corner displays the interstation paths involved in computing the corresponding surface wave map. The red triangles mark the positions of the seismic stations. 


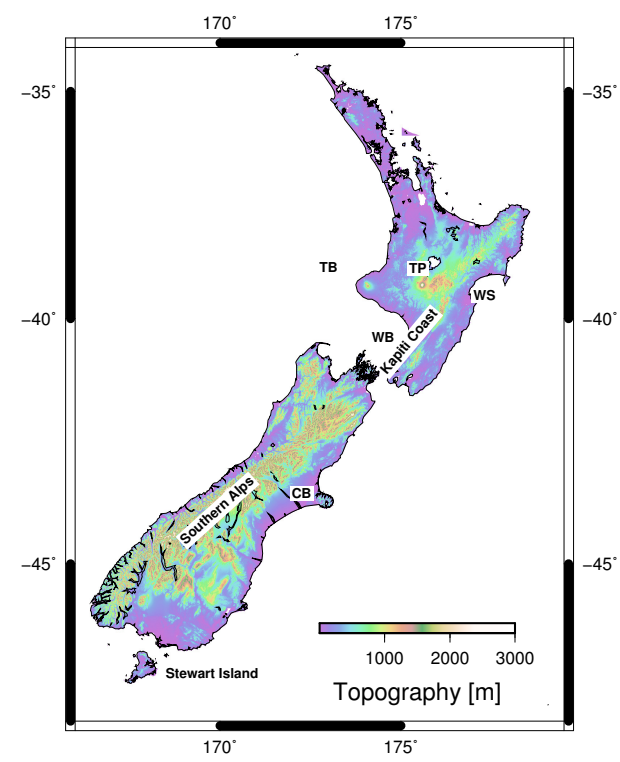

Figure 3.10 Topography of New Zealand. Locations marked on the map and referred to in the text are: Lake Taupo (TP), Taranaki Basin (TB), Wairoa Syncline (WS), Wanganui Basin (WB) and the Canterbury Basin (CB).

incorrect seismograph wiring at either station — "reversed polarities" - are the main reasons why it is more difficult to measure phase velocities than group velocities [Lin et al., 2008].

To resolve the integer ambiguity, we choose the phase velocity dispersion curve that lies closest to a reference curve [Lin et al., 2008], which in this case is the Rayleigh wave phase velocity curve obtained for the North Island by Brisbourne and Stuart [1998] using surface wave recordings from teleseismic events. Although this curve will be less suitable for the South Island, it is still accurate enough to discriminate between dispersion curves of one cycle difference, given the wavelengths and velocities involved. To the best of our knowledge there has been no previous study of Love wave dispersion curves in New Zealand and we therefore use the Rayleigh wave dispersion curve of Brisbourne and Stuart [1998] increased by $9 \%$ as the Love wave reference curve following Lin et al. [2008].

In order to identify dispersion curves contaminated by $180^{\circ}$ phase shifts resulting from reversed polarities, we compare the measured dispersion curves to theoretical dispersion curves obtained from overdamped surface wave maps. We expect the dispersion curves measured from cross-correlations to lie within $10 \%$ of those obtained from the 
overdamped surface wave maps, which enables us to find outliers. Choosing a $10 \%$ margin is somewhat arbitrary, but visual inspection of the dispersion curve dataset shows that it is wide enough to include most dispersion curves but small enough to reject outliers.

As mentioned in Section 3.1, the measurement of dispersion curves proves more difficult for Love waves than for Rayleigh waves even when integer ambiguity and reversed polarity cases have been addressed. Possibly due to different excitation mechanisms [e.g. Stehly et al., 2009] for Love and Rayleigh waves and higher incoherent noise amplitudes on horizontal components, Love waves have lower signal-to-noise ratios (SNR) than Rayleigh waves. Furthermore, deviations of the horizontal seismograph components from true north and multipathing of Rayleigh waves can both lead to spurious arrivals on the seismogram's transverse component and thereby obscure the Love wave arrival [Goforth and Herrin, 1979]. This reduces the number of reliable Love wave dispersion curves, and produces a concomitant reduction in path coverage compared to that of Rayleigh waves, as illustrated in Figure 3.7. Consequently, features seen in the Love wave phase velocity maps will in general be less constrained laterally than in the corresponding Rayleigh wave phase velocity maps.

Love waves have higher sensitivity to shallower structures than Rayleigh waves of the same period as can be seen by comparing the sensitivity kernels illustrated in Figure 3.11 and 3.12. At crustal scales, Love waves of period $T$ sample similar depths to Rayleigh waves of period $T-4 \mathrm{~s}$ to $T-5 \mathrm{~s}$ [Lin et al., 2008]. Accordingly, Love wave maps at $13 \mathrm{~s}$ and $18 \mathrm{~s}$ period are expected to show similar features to Rayleigh wave maps at $8 \mathrm{~s}$ and $13 \mathrm{~s}$ period. The Love wave map at $23 \mathrm{~s}$ period shows less similarities to the $18 \mathrm{~s}$ Rayleigh wave map, as a likely consequence of the poor path coverage.

Sensitivity kernels for Rayleigh wave group velocities (Figure 3.9) resemble those for Rayleigh wave phase velocities (Figure 3.11) at periods $\sim 5$ s shorter. Hence the Rayleigh wave phase velocity map for the $8 \mathrm{~s}, 13 \mathrm{~s}$ and $18 \mathrm{~s}$ period phase velocities show very similar features to the Rayleigh wave group velocity maps at $13 \mathrm{~s}, 18 \mathrm{~s}$ and $23 \mathrm{~s}$ period. The three-dimensional sensitivity to changes in earth properties, such as shear velocity, differ between group and phase velocities with group velocities having higher sensitivity to off-great-circle path and shallow variations in shear-velocity than phase velocities [Dahlen and Zhou, 2006]. However, the difference in lateral sensitivity is not particularly evident in our surface wave maps, suggesting that differences due to this effect are only marginal for the period range and path lengths considered in this study (cf. Figure 3.11 and Figure 3.9). The sensitivity to deeper structures at identical periods is a significant advantage of phase velocities over group velocities. The $23 \mathrm{~s}$ period Rayleigh wave phase velocity map therefore mainly images slow uppermost mantle [Haines, 1979; 
Seward et al., 2009] and consequently the slow velocities (phase: $\sim 3 \mathrm{~km} / \mathrm{s}$; group: $\sim 2.7$ $\mathrm{km} / \mathrm{s}$ ) beneath the North Island are more confined to the central North Island in the Rayleigh wave phase velocity map than in the Rayleigh wave group velocity map. In the South Island, the $23 \mathrm{~s}$ period Rayleigh wave group velocity map resembles the $18 \mathrm{~s}$ period Rayleigh wave phase velocity map, both showing low velocities (phase: $\sim 3.3$ $\mathrm{km} / \mathrm{s}$; group: $\sim 2.9 \mathrm{~km} / \mathrm{s}$ ) of the deep crustal root beneath the Southern Alps surrounded by faster mantle lithosphere (phase: $\sim 3.6 \mathrm{~km} / \mathrm{s}$; group: $\sim 3.2 \mathrm{~km} / \mathrm{s}$ ).

The results for the Rayleigh and Love wave phase velocities are shown in Figure 3.11 and 3.12 for periods of $8 \mathrm{~s}, 13 \mathrm{~s}, 18 \mathrm{~s}$ and $23 \mathrm{~s}$. Slow velocities (Rayleigh: $\sim 2.6 \mathrm{~km} / \mathrm{s}$; Love: $\sim 2.9 \mathrm{~km} / \mathrm{s}$ ) beneath the North Island revealed by the $8 \mathrm{~s}$ Rayleigh and Love wave map coincide with the locations of deep sedimentary basins: the Wairoa Syncline to the east and the Taranaki and Wanganui Basin to the west (Figure 3.10). On the South Island, the $8 \mathrm{~s}$ Love wave map shows slow velocities of $\sim 3.1 \mathrm{~km} / \mathrm{s}$ within the Canterbury Basin. Rayleigh waves at this period are sensitive to deeper structures and therefore slow velocities $(\sim 2.8 \mathrm{~km} / \mathrm{s})$ in the $8 \mathrm{~s}$ Rayleigh wave map are more likely to show the metasedimentary rocks of the basement Pahau Terrane (Figure 3.13), whose surface extent also corresponds well to the slow velocities extending along the East Coast of the North Island and the northern South Island in the $13 \mathrm{~s}$ and $18 \mathrm{~s}$ Rayleigh and Love wave maps (Rayleigh: $\sim 2.9 \mathrm{~km} / \mathrm{s}$; Love: $\sim 3.3 \mathrm{~km} / \mathrm{s}$ ). Fast velocities extending from the Kapiti Coast towards Lake Taupo (Figure 3.10) as seen in the $8 \mathrm{~s}$ and $13 \mathrm{~s}$ Rayleigh wave maps and the $13 \mathrm{~s}, 18 \mathrm{~s}$ and $23 \mathrm{~s}$ Love wave map are here interpreted to show the northern extent of the greywacke-dominated Rakaia Terrane (Figure 3.13) which extends southward in the central and southern South Island.

Overall fast velocities in the southwest of the South Island displayed in all Rayleigh wave phase velocity maps appear to correlate spatially to the location of the southern part of the Median Batholith (Figure 3.13), which consists of gabbroic and granitic plutons. While similar features can be observed in our Rayleigh wave group velocity maps, the Love wave phase velocity maps and the Rayleigh wave group velocity maps of Lin et al. [2007] exhibit comparatively slow velocities in this area. We propose that these ostensibly slower velocities are a damping effect, in which the velocity model is biased towards the mean velocity due to poor path coverage (see Section 2.4.1). The same effect might be responsible for the slow velocities $(\sim 3.5-3.6 \mathrm{~km} / \mathrm{s})$ on the West Coast of the South Island seen in the $13 \mathrm{~s}$ and $18 \mathrm{~s}$ Love wave maps. At longer wavelengths, these slow velocities merge with the slow velocities of the deep crustal root beneath the Southern Alps as seen in the $18 \mathrm{~s}$ and $23 \mathrm{~s}$ Love and Rayleigh wave maps.

The extent of fast velocities to the west of New Zealand at the $18 \mathrm{~s}$ and $23 \mathrm{~s}$ period is simi- 

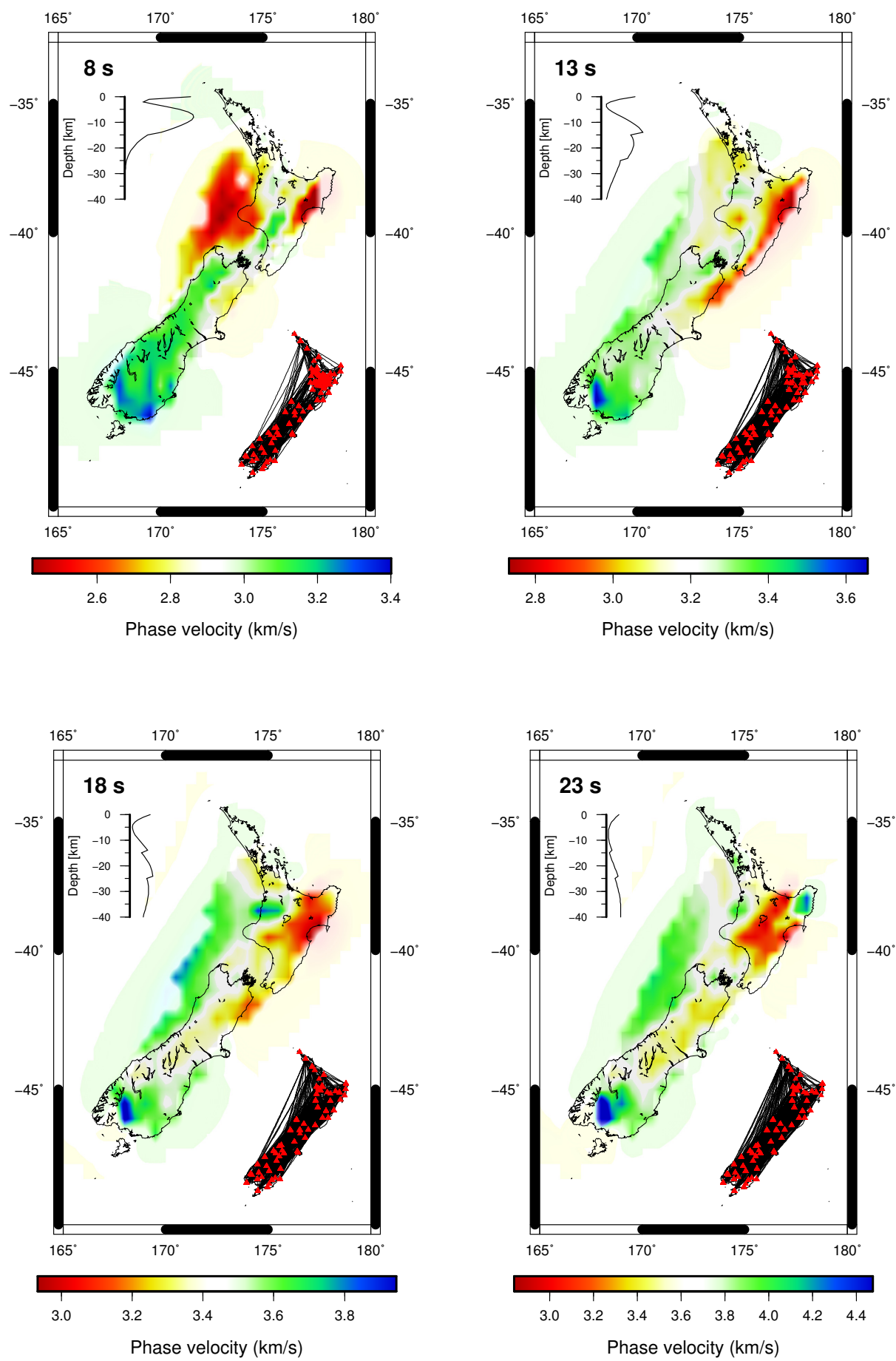

Figure 3.11 Rayleigh wave phase velocity maps at the $8 \mathrm{~s}, 13 \mathrm{~s}, 18 \mathrm{~s}$ and $23 \mathrm{~s}$ period. Colours outside the area within which resolution is better than $100 \mathrm{~km}$, are toned down. The inset in the upper left corner shows the sensitivity kernel of phase velocity to changes in S-velocity $(\partial \mathrm{C} / \partial \mathrm{Vs})$ with respect to depth at the corresponding period calculated for PREM [Dziewonski and Anderson, 1981]. The inset in the lower right corner displays the interstation paths involved in computing the corresponding surface wave map. The red triangles mark the positions of the seismic stations. 
lar to that of the northern part of the Median Batholith. However, the sensitivity kernels for these periods indicate that the fast velocities are manifestations of both crustal and upper mantle structures and therefore cannot only be attributed to plutonic crustal rocks. Love wave maps also exhibit faster velocities in this area at the $18 \mathrm{~s}$ and $23 \mathrm{~s}$ periods but impose fewer constraints on their lateral distribution due to poor path coverage. Both, Rayleigh and Love wave maps at $23 \mathrm{~s}$ period exhibit similarities with the 3D P-velocity structure at $38 \mathrm{~km}$ depth in the northern South Island, inferred by Eberhart-Phillips and Bannister [2010] from traveltime tomography using active and passive source data. They interpreted the high velocities in the western part of this region as the high density remnants of plutonic rocks that are present in the overlying crust. The lower velocities to the east are attributed by Eberhart-Phillips and Bannister [2010] to thickened crust extending down to $40 \mathrm{~km}$ depth as a result of the subducting Pacific plate pulling down lower-crustal material.

Love wave maps for the $18 \mathrm{~s}$ and $23 \mathrm{~s}$ periods mostly resemble the Rayleigh wave map at $18 \mathrm{~s}$ period. However, the slow velocities in the southern South Island exhibited by the Love wave velocities at $23 \mathrm{~s}$ period appear as a fast velocity feature in the Rayleigh wave maps. A likely explanation for this marked difference is the higher sensitivity to upper crustal structures of Love waves at this period with regard to Rayleigh waves. This may also explain the differences between Rayleigh and Love wave maps on the North Island. The Love waves at $23 \mathrm{~s}$ period display fast velocities of the Rakaia Terrane whereas Rayleigh waves show slow velocity features in the lower crust and upper mantle beneath the North Island.

\subsection{Discussion and conclusion}

The overall consistency of surface maps for Rayleigh and Love waves and the good correlation with known geological features demonstrates the feasibility and stability of inferring Rayleigh and Love wave phase velocities from ambient seismic noise crosscorrelation functions. The north-south smearing in our inversion results due to the uneven path-coverage (Figure 3.4) is compensated for, to some extent, by the basement terranes' orientation approximately along the strike of New Zealand. Imaging stronger lateral variations along the strike of New Zealand would be more problematic. Similar smearing effects were reported by Li et al. [2010] from checkerboard tests for the inversion of Rayleigh wave group velocities in Italy, whose elongated mainland is comparable in size and shape to New Zealand. However the inclusion of seismic stations in Sardinia, 

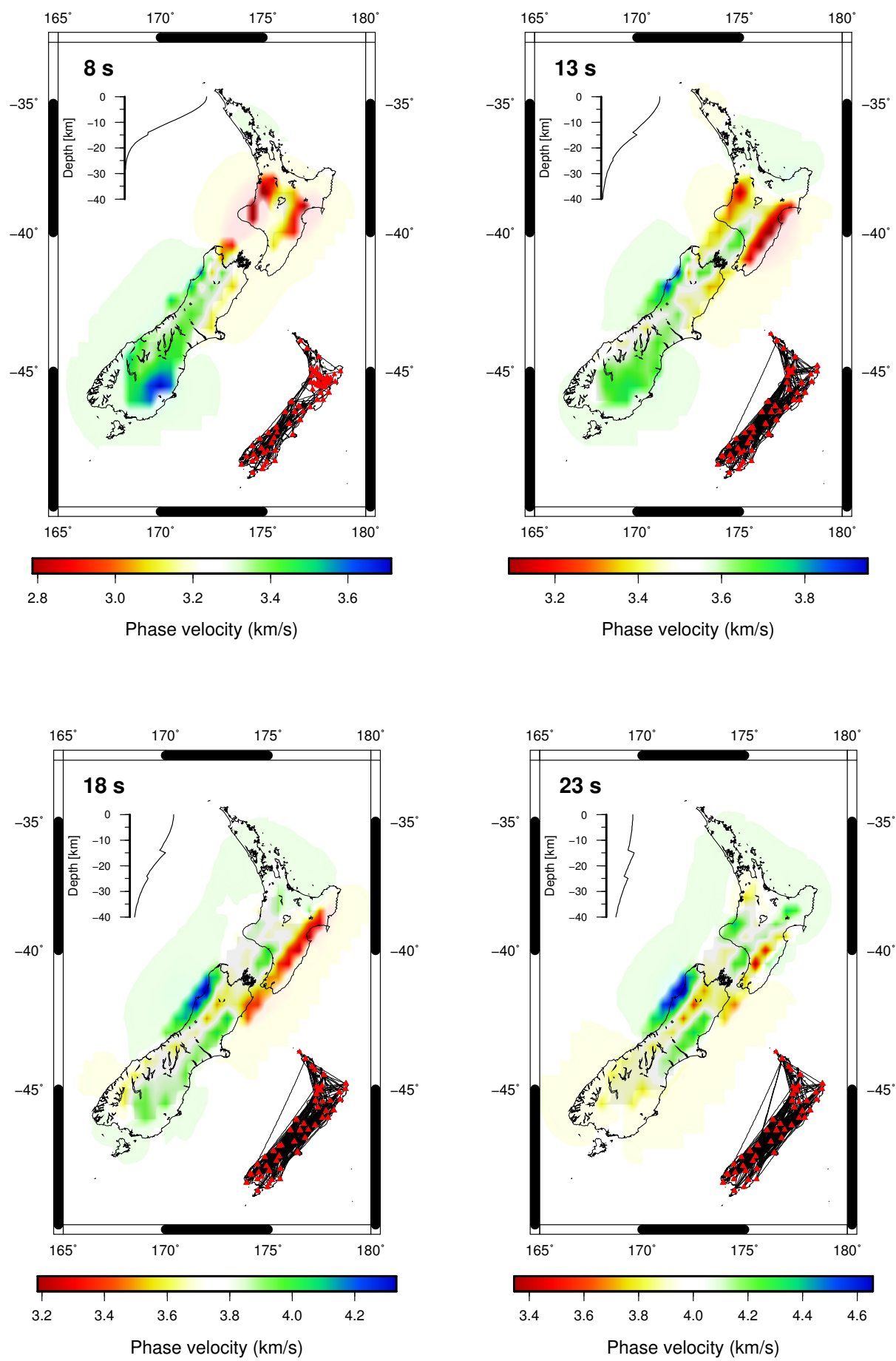

Figure 3.12 Love wave phase velocity maps at the $8 \mathrm{~s}, 13 \mathrm{~s}, 18 \mathrm{~s}$ and $23 \mathrm{~s}$ period. Colours outside the area within which resolution is better than $100 \mathrm{~km}$, are toned down. The inset in the upper left corner shows the sensitivity kernel of phase velocity to changes in S-velocity $(\partial \mathrm{C} / \partial \mathrm{Vs})$ with respect to depth at the corresponding period calculated for PREM [Dziewonski and Anderson, 1981]. The inset in the lower right corner displays the interstation paths involved in computing the corresponding surface wave map. The red triangles mark the positions of the seismic stations. 


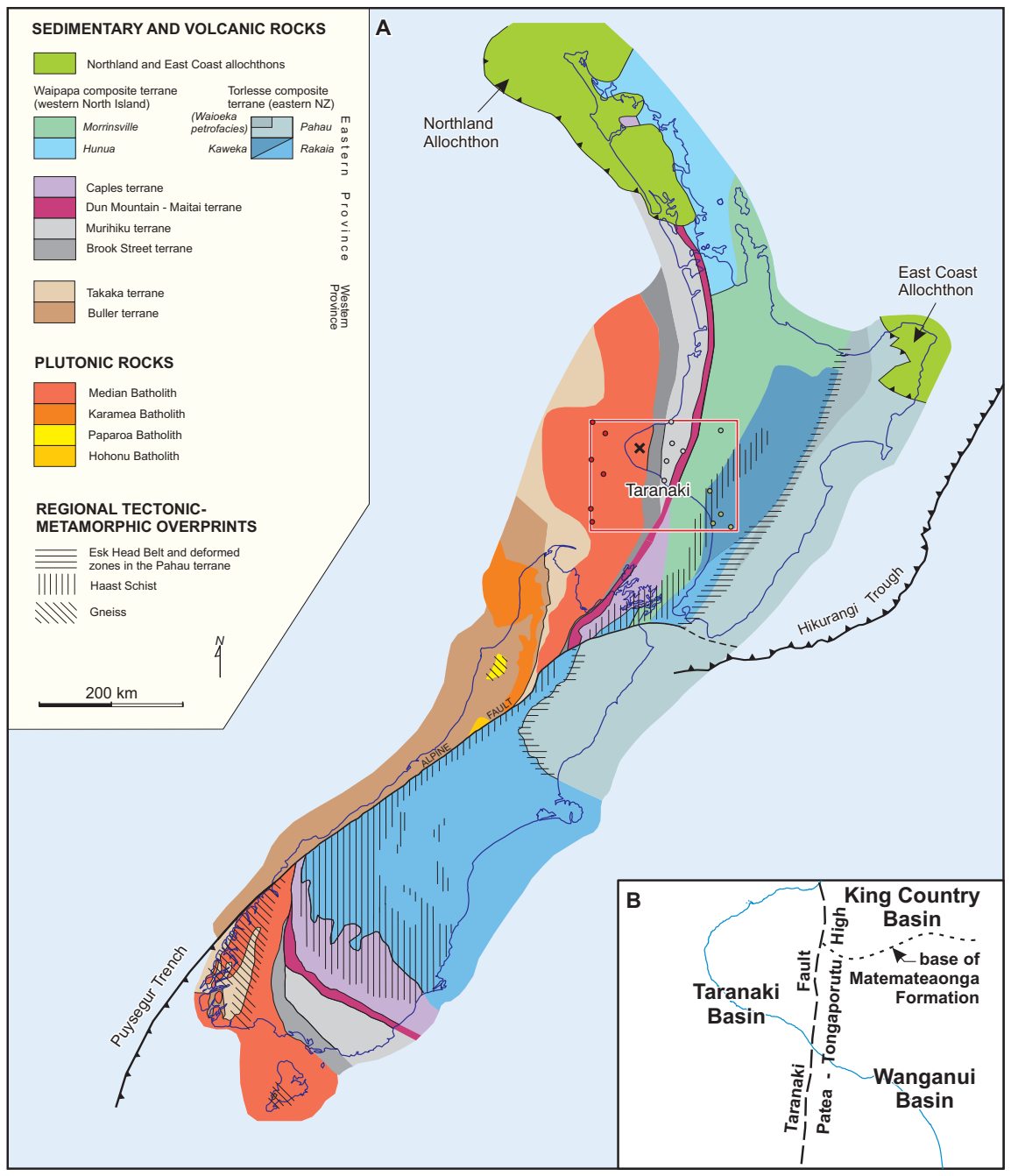

Figure 3.13 New Zealand's basement geology [Mortimer, 2004] 
Sicily and neighbouring European countries in that case ameliorated the lateral resolution problems. In New Zealand, continuous recordings from the most recent deployment of 29 broadband ocean-bottom seismometers off the coast of the southern and central South Island [Marine Observations of Anisotropy Near Aotearoa; MOANA Collins et al., 2010] are likely to improve the lateral extend and resolution of surface wave maps significantly.

In the light of several theoretical derivations of the relation between ambient noise cross-correlations and the Green's Function between two seismographs the stability of ambient noise tomography seems somewhat surprising. For example, Snieder [2004] and Derode et al. [2003] assumed an isotropic distribution of sources and Sánchez-Sesma and Campillo [2006] assumed equipartitioning, such that all modes in a medium carry the same energy, to demonstrate the feasibility of retrieving the exact Green's Function from ambient noise cross-correlations. However, none of these requirements are likely to be fully met in a realistic environment. Tsai [2010] showed how parts of the Green's Function can be retrieved even in the absence of equipartition. Yao and Van Der Hilst [2009] explored the influence of non-isotropic noise-source distributions on the accuracy of phase velocity measurements in numerical experiments and demonstrated that the inference of isotropic wave speed variations from ambient noise correlations is robust if the azimuthal distribution of noise sources varies smoothly and if long-duration (i.e. several months) noise recordings are used for the analysis. Tsai [2009] established numerically that the influence of a strong stationary noise source can bias surface wave phase and group velocity measurements considerably. The pre-processing we employ (see Section 2.3.1) reduces the effect of stationary noise sources and beamforming results from the central North Island (see Chapter 5.2) show, at least for the North Island, that the azimuthal distribution of noise sources varies smoothly, which explains the robustness of our surface wave maps.

The feasibility of ambient noise tomography has been demonstrated for regions with distinctly different ambient noise source distributions. For example, Yao et al. [2006] and Shapiro et al. [2005] successfully employed ambient noise tomography to compute surface wave maps of southeast Tibet and California respectively, where noise source distributions are, at least for some period ranges, markedly heterogeneous [Yao and Van Der Hilst, 2009; Stehly et al., 2006]. Gudmundsson et al. [2007] and Brenguier et al. [2007] demonstrated ambient noise tomography on Iceland and La Réunion Island, both of which are surrounded by sea and therefore subject to much more azimuthally homogeneous noise source distributions than seismic networks in continental environments. 
The longer the interstation paths, the more surface waves will be affected by multipathing. Due to the minimum interstation distance criterion of two wavelengths (Chapter 2.3.3), this means that surface wave maps at longer periods are more affected by multipathing effects than those at shorter periods. Lin and Ritzwoller [2010] showed how to construct lateral sensitivity kernels for Rayleigh waves empirically using phase velocity maps inferred from ambient noise correlations and a very dense seismic array. It remains to be determined how lateral sensitivity kernels can be estimated for sparse seismic networks and if using them will improve the phase velocity maps of New Zealand from ambient noise correlations. However, the incorporation of surface waves from teleseismic earthquakes, extending the surface wave analysis to longer periods will probably require more sophisticated approaches than the straight ray method adopted in this study.

In summary, calculating surface wave maps for Rayleigh and Love waves yields useful information pertaining to regional crustal structure and highlights the utility of ambient noise tomography. When interpreted in combination with the corresponding sensitivity kernels, surface wave maps provide furthermore a first-degree indicator for the depth extent of basement structures. However, for more quantitative constraints on the threedimensional extent of crustal and uppermost mantle structures, an inversion of the surface waves for 3D or pseudo-3D S-velocity structure is necessary, as is demonstrated in the following chapters. In other words, the inversion for the lateral distribution of Rayleigh and Love wave velocities should be treated as an intermediate step in the analysis of surface wave dispersion measurements, and where station coverage, noise source characteristics and resolution permits, it is likely that $\mathrm{S}$-wave modelling will provide greater geophysical insight. In Chapter 4 we invert Rayleigh and Love wave dispersion curves for path-averaged 1D S-velocity profiles beneath the Northland Peninsula. In Chapter 5 we combine the methods used in this chapter and in Chapter 4 to infer the crustal pseudo-3D S-velocity structure of the central North Island from Rayleigh and Love wave dispersion curve measurements. 


\section{Shear-velocity structure of the Northland Peninsula, New Zealand, inferred from ambient noise correlations ${ }^{1}$}

Ambient noise correlation has been successfully applied in several cases to regions with dense seismic networks whose geometries are well-suited to tomographic imaging. The utility of ambient noise correlation-based methods of seismic imaging where either network or noise-field characteristics are less ideal has yet to be fully demonstrated. In this study, we focus on the Northland Peninsula of New Zealand using data from five seismographs deployed in a linear pattern parallel to the direction from which most of the ambient noise arrives. Shear-wave velocity profiles computed from Rayleigh and Love wave dispersion curves using the Neighbourhood Algorithm are in good agreement with the results of a previous active source refraction experiment and a teleseismic receiver function and surface wave analysis. In particular, we compute a path-averaged Moho depth of $\sim 28 \mathrm{~km}$ along a $\sim 250 \mathrm{~km}$ profile. The use of both Rayleigh and Love wave measurements enables us to estimate the degree of radial anisotropy in the crust, yielding values of $2-15 \%$. These results demonstrate that ambient noise correlation methods provide useful geophysical constraints on lithospheric structure even for non-optimal network geometries and noise-field characteristics.

\subsection{Introduction}

As discussed in Chapter 1 and 2, cross-correlating long intervals of ambient seismic noise between two seismographs has been shown to yield under certain circumstances an estimate of the general Green's function for the station pair, and its surface wave part

\footnotetext{
${ }^{1}$ Previously published by Behr et al., JGR, 2010.
} 
in particular [e.g. Weaver and Lobkis, 2001b, 2004, 2005, 2001a; Derode et al., 2003; Larose et al., 2006; Snieder, 2004]. Utilising the dispersive character of surface waves to create tomographic images of the 2-D velocity distributions for different central frequencies is becoming an almost routine tool in seismology [Shapiro and Campillo, 2004; Yang et al., 2007; Lin et al., 2008] and 3-D analysis is now increasingly common [e.g. Yao et al., 2006; Liang and Langston, 2009; Nishida et al., 2008]. In theory, an isotropic distribution of noise sources around a station pair or a highly heterogeneous medium is required to reconstruct the full Green's function [Sánchez-Sesma and Campillo, 2006; Wapenaar, 2006; Larose et al., 2006; Tsai, 2009]. In practice, however, even if these conditions are not fulfilled, cross-correlations of diffuse waves can yield a meaningful estimate of the medium's Green's function [Paul et al., 2005; Yang et al., 2007].

In their pilot study, introduced in Chapter 1 and 4, Lin et al. [2007] demonstrated ambient noise correlation tomography to be feasible in New Zealand using one year of continuous vertical-component data recorded at 42 permanent broadband stations. The 2-D group-velocity maps resulting from that study, for periods between 8 and $23 \mathrm{~s}$, agree well with known geological features [cf. Mortimer, 2004]. Due to New Zealand's elongate geometry, however, observations at periods longer than $\sim 23 \mathrm{~s}$ are restricted to alongstrike, approximately northeast-southwest-oriented paths, making a New Zealand-wide 3-D inversion difficult. Because of its oceanic isolation, long shoreline and consequent high wave heights [Laing, 2000; Pickrill and Mitchell, 1979; Gorman et al., 2003a], New Zealand serves as an excellent target for investigating seismological imaging techniques using ambient noise sources [Brooks et al., 2009a, b].

Although 2-D velocity maps computed at different periods are instructive and serve as a useful means of verifying cross-correlation results, more practical benefit lies in the computation of 2-D velocity-depth models or fully 3-D volumes, and several studies have recently addressed this matter. For example, Brenguier et al. [2007] used Rayleigh wave group velocities measured from cross-correlation to construct a 3-D model of the Piton de la Fournaise volcano on La Réunion island. Yao et al. [2008] inverted Rayleigh wave phase velocities for 3-D shear-velocity structure beneath the Tibetan plateau, and Moschetti et al. [2010] used Love and Rayleigh wave phase and group velocities to infer the 3-D anisotropic crustal and uppermost mantle shear-velocity structures of the western USA. All three of these studies involved the construction of local dispersion curves at grid-points spanning 2-D velocity maps for different periods. These dispersion curves were subsequently inverted for 1-D shear-velocity profiles using Monte Carlo inversion methods to construct a final pseudo-3-D model.

Here we examine the applicability of shear-velocity profiling in an environment charac- 
terised by a high-amplitude, anisotropic noise field, using data recorded during short-term deployments on the Northland Peninsula, New Zealand. To do so, we compare 1-D shearvelocity profiles obtained from noise cross-correlation to the results of previous studies employing active sources and teleseismic events.

\subsubsection{Data}

We use data recorded during the NORthland Deployment [NORD; Duclos, 2005, see Figure 4.1] to test our processing and inversion procedures. The NORD deployment comprised five broadband stations (three Guralp-CMG 40T sensors, one Guralp-CMG 3ESP and one Streckeisen STS-2 sensor; Orion and Quanterra data loggers) aligned along a northeast-southwest-trending axis that were operated between September 2002 and February 2004 for durations varying from eleven to sixteen months.

\subsubsection{Geology}

Most of New Zealand's present-day continental crust is thought to have formed by terrane accretion in the late Cretaceous when it was part of Gondwana [Mortimer, 2004; Sutherland, 1999a]. Rifting prior to the Late Cretaceous created the Northland Basin to the west of the Northland Peninsula followed by subsidence of the entire Northland region from the Late Oligocene until the Early Miocene. Between 25 and $22 \mathrm{Ma}$, sediments and oceanic crust were obducted and subsequently emplaced westwards to form what is known as the Northland Allochthon, causing a rebound of the basement. At approximately the same time, a subduction zone developed off the east coast of Northland creating two volcanic chains, one along the east coast and the other along the west coast [Herzer, 1995; Spörli, 1989; Isaac et al., 1994].

Northland's basement rock can be divided into three terranes [Mortimer, 2004]. The Murihiku Terrane in the west consists of sedimentary rocks and conglomerates that were deposited in forearc or backarc basins. To the east of the Murihiku Terrane lies the Maitai Terrane, an ophiolite belt overlain by volcaniclastic sedimentary sequences that formed in a near-arc setting. The east coast of Northland is mainly part of the Bay of Islands Terrane, which comprises basalts, limestones, sandstones and mudstones formed in trench and trench-slope environments. Large portions of these terranes are overlain by the Northland Allochthon. 


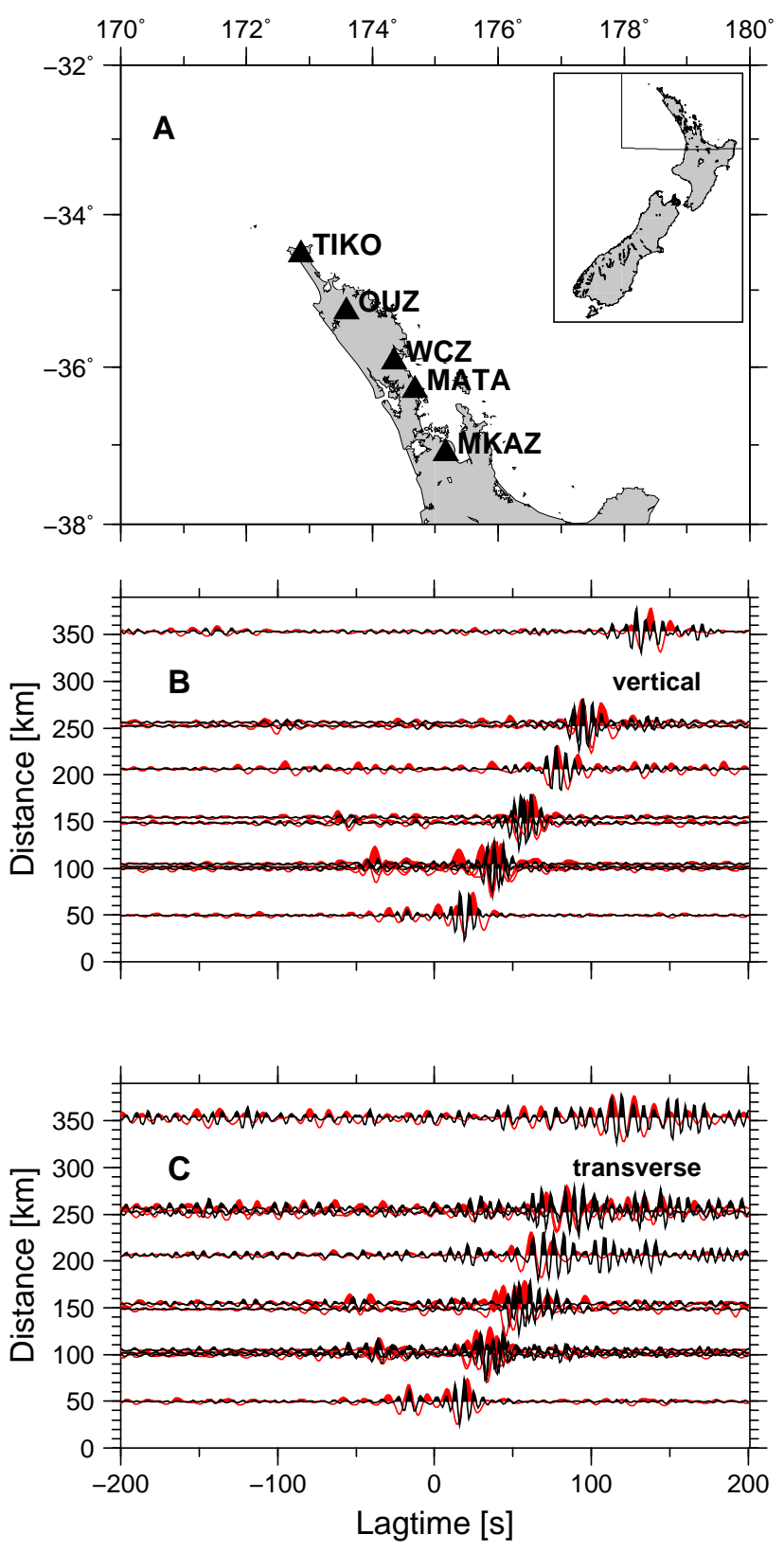

Figure 4.1 (A) Stations used in the Northland deployment (NORD); (B) Cross-correlation results in the 5-10 s period band (black) and 10-20 s period band (red) for the vertical component; strong asymmetries in amplitude imply dominant sources towards the northwest of the deployment; (C) the same as (B) but for the transverse component. 
There are volcanological and geochemical indications of present-day mantle melting and partial melting in the crust in some parts of the Northland Peninsula [Hoke and Sutherland, 1999; Smith et al., 1993]. Occurrence of rhyolite outcrops and geothermal activity near the station at Omahuta (OUZ) has been suggested to be due to rhyolite intrusions in the crust [Heming, 1980]. High ${ }^{3} \mathrm{He} /{ }^{4} \mathrm{He}$ values and low upwelling rates indicate mantle melting beneath the Peninsula [Hoke and Sutherland, 1999; Huang et al., 1997].

\subsubsection{Previous studies}

Two principal seismological experiments have been conducted to determine crustal and upper mantle structure beneath the Northland Peninsula. Using wide-angle seismic refraction data, Stern et al. [1987] determined a $25 \pm 2 \mathrm{~km}$ average crustal thickness with P-wave velocities ranging between 5.3 and $5.9 \mathrm{~km} / \mathrm{s}$ in the upper crust, $6.2 \mathrm{~km} / \mathrm{s}$ in the lower crust and upper mantle velocities increasing from $7.6 \mathrm{~km} / \mathrm{s}$ at the Moho to $7.9 \mathrm{~km} / \mathrm{s}$ at $40 \mathrm{~km}$ depth. Horspool et al. [2006] jointly inverted teleseismic receiver functions and teleseismic surface wave phase velocity dispersion curves to derive shear-velocity profiles. They inferred crustal thicknesses ranging from $26 \pm 1 \mathrm{~km}$ to $29 \pm 1 \mathrm{~km}$ and average shear-velocities of 3.4 to $3.6 \mathrm{~km} / \mathrm{s}$ in the upper crust, 3.6 to $4.0 \mathrm{~km} / \mathrm{s}$ in the lower crust and $4.2 \pm 0.1 \mathrm{~km} / \mathrm{s}$ in the upper mantle. Furthermore, they found manifestations of two low-velocity layers, one in the upper crust between stations Omahuta (OUZ) and Waipu Caves (WCZ) and the other in the upper mantle beneath Moumoukai (MKAZ).

The NORD deployment's linear geometry is not ideal for ambient noise tomography, but the earlier studies conducted nearby make this region a useful testing environment for inverting surface wave dispersion curves from azimuthally anisotropic noise cross-correlations to obtain shear-velocity profiles. Moreover the availability of threecomponent data enables us to examine differences in Rayleigh and Love wave propagation.

\subsection{Method}

\subsubsection{Preprocessing and velocity measurements}

Our processing scheme is similar to that described by Bensen et al. [2007] and used previously in a New Zealand setting by Lin et al. [2007]. The instrument response for each 
station as well as the mean and the trend are first removed, the signals are decimated to one $\mathrm{Hz}$ sampling frequency if necessary and the traces are then cut to a uniform length of one day. Due to various operational factors, the number of resulting day-long traces for each of the five stations varies between 149 (MKAZ) and 535 (WCZ). The spectra for all three components for every available day and station pair are whitened and signals with large amplitudes in a typical earthquake period band of 15-50 s are down-

weighted. Cross-correlation of all resulting traces for each day and subsequent stacking of the cross-correlation functions over the whole time span of the data set yields an estimate of the causal and anticausal portions of the Green's function between pairs of stations at positive and negative lag times respectively. To compensate for amplitude differences between causal and anticausal part, a presumed consequence of heterogeneous noise source distributions, we add to each cross-correlation function its time-reversed trace to create what is known as the symmetric-component cross-correlation.

Under the assumptions that Love- and Rayleigh-waves are polarised in the transverse and radial-vertical planes respectively, and that they are dominated by fundamental modes, we measure Rayleigh and Love wave group and phase velocities using the multiple-filter technique ('FTAN') of Levshin et al. [1992]. In phase velocity studies elsewhere the total number of cycles has generally been determined by comparing the FTAN output with a reference curve obtained from previous results [e.g. Lin et al., 2008]. We use the results of Horspool et al. [2006] as a reference curve for Rayleigh waves. As a suitable reference curve for Love waves doesn't exist for the Northland Peninsula we follow the suggestion of Lin et al. [2008] and use the Rayleigh wave reference curve with velocities increased by $9 \%$.

\subsubsection{Error analysis}

To assess the reliability of our measurements, we adapt the data selection and error analysis methods proposed by Bensen et al. [2007] and Lin et al. [2007] to account for the shorter recording times and non-ideal network geometry. Dispersion curves are measured between periods of $5 \mathrm{~s}$ and a cutoff corresponding to twice the maximum wavelength $T_{\max }=\Delta /\left(2 * v_{\max }\right)$, where $\Delta$ is the the station separation and $v_{\max }$ is the assumed maximum surface wave velocity, in order to avoid spurious signals from interference between the causal and anticausal parts [Shapiro et al., 2005; Lin et al., 2007].

Signal-to-noise ratios are defined here as the ratio between the maximum amplitude of the symmetric-component cross-correlation within a predefined signal window and the root-mean-square of the trailing noise [e.g. Bensen et al., 2008; Yang et al., 2007]. We 
define the signal window as the part of the symmetric-component cross-correlation that lies within the expected minimal and maximal traveltime of surface waves between a station pair and add a buffer of $500 \mathrm{~s}$ to the end of the window $\left(t_{\min }=\Delta / 4[\mathrm{~km} / \mathrm{s}]\right.$, $t_{\max }=500 \mathrm{~s}+\Delta / 1.5[\mathrm{~km} / \mathrm{s}]$ ). The signal after $t_{\max }$ is regarded as incoherent noise (Y. Yang, personal communication, 2009).

As noise cross-correlations are expected to be largely independent of the particular time at which the noise was recorded [see, for example Yao et al., 2006], we can stack daily crosscorrelations over different time-periods and compute uncertainties from differences in the corresponding dispersion curves. We stack 100 days of cross-correlations by shifting the stacking window in increments of 30 days, which results in up to 12 sub-stacks.

\subsubsection{Inversion for S-velocity profiles}

Finding a velocity model that corresponds to an observed surface wave dispersion curve is a highly nonlinear optimisation problem [e.g. Dunkin, 1965]. A common approach is to linearise the problem in the vicinity of a starting model and apply an iterative gradient method to find the best-fitting model [e.g. Juliá et al., 2000]. This approach suffers, however, from several well-known problems [see, for example, Parker, 1994]. First, the final model depends strongly on the starting model. Second, it is difficult to ascertain whether the final model corresponds to a local or a global minimum, and hence to quantify the solution's uncertainty. And third, gradient methods that guarantee convergence to a final model are known to be slow. The increased availability of computational power in recent years has made the use of direct search methods feasible, even when the parameter space is large.

To overcome some of the problems associated with linearised approaches we have adopted the Neighbourhood Algorithm (NA), a quasi-random direct search method similar to genetic algorithms or simulated annealing [Sambridge, 1999a]. Sampling of the parameter space is steered by ranking randomly generated models according to a usersupplied criterion. Wathelet et al. [2004] successfully employed the NA in the inversion of surface wave dispersion curves for shallow shear-velocity profiles (depth $<130 \mathrm{~m}$ ). Their results were consistent with data from active-source experiments and a borehole log for the same site. Snoke and Sambridge [2002] compared the NA to an iterative gradient method for the inversion of surface wave dispersion curves in southeast Brazil and found that the best-fitting models obtained with the two techniques agreed well. However, because the NA sampled larger portions of the parameter space, additional information was obtained about the ensemble of models that fully fit the data. 


\subsection{Results}

Of the 10 possible pairs of stations and corresponding travel paths, we focus on two between Tikorangi (TIKO) and Matakana (MATA) and TIKO and Waipu Caves (WCZ) to invert for shear-velocity profiles using the NA. These two paths span the second and third greatest distances and have signal-to-noise ratios higher than those of other station pairs at comparable distances. They further share very similar travel paths. For the remaining cross-correlation functions it either proved difficult to obtain physically reasonable dispersion curves or the depth resolution was overly restricted by the length of the interstation distance to warrant detailed analysis (see Section 2.3.3).

Figure 4.3 shows the velocity measurements for TIKO-MATA and TIKO-WCZ respectively. As the two paths sample similar regions we would anticipate obtaining similar dispersion curve measurements. However, the group velocities between the two station pairs differ substantially for Rayleigh waves (RG) at periods greater than $15 \mathrm{~s}$ and for Love waves (LG) over the whole period range considered. The Rayleigh wave phase (RP) and Love wave phase (LP) velocity measurements, in contrast, are very similar for both station-pairs and to the reference curves taken from Horspool et al. [2006]. To ensure consistency of our phase and group velocity measurements, we checked that measured group velocitiy dispersion curves and the group velocity dispersion curves from phase velocity measurements were identical (i.e. $V_{g}=\partial \omega / \partial k$ ). A possible explanation for the discrepancy in group velocities is given by Tsai [2009] who showed that a strong noise source anisotropy off the interstation path can reduce the group velocity considerably while leaving the phase-velocity almost unchanged. The fact that such a source only affects the group velocities between TIKO and WCZ could indicate a disturbing source close by station WCZ. Differences in signal-to-noise ratios for Love and Rayleigh waves for the secondary microseismic band (5-10 s) suggest different source mechanisms, as proposed by Longuet-Higgins [1950]. While Love waves in the primary microseism band (10-20 s) are excited by direct coupling of ocean waves to the ocean bottom, they are mainly a result of scattering of Rayleigh waves in the secondary microseism band. Therefore the higher signal-to-noise ratios for TIKO-WCZ compared to TIKO-MATA in the primary microseism band (Figure 4.2) support the suggestion of a localised source close by station WCZ.

We parameterise the model-space using four layers over a half space, three for the crust and one for the upper mantle. The shear- and P-velocities, layer thickness and Poisson's ratios of each layer are allowed to vary substantially while the density is fixed. The misfit is a least squares misfit defined as $\sqrt{\sum_{i=1}^{n_{F}}\left(x_{\text {data }}-x_{\text {calc }}\right)^{2} /\left(x_{\text {data }}^{2} * n_{F}\right)}$, where $x_{\text {data }}$ is the 


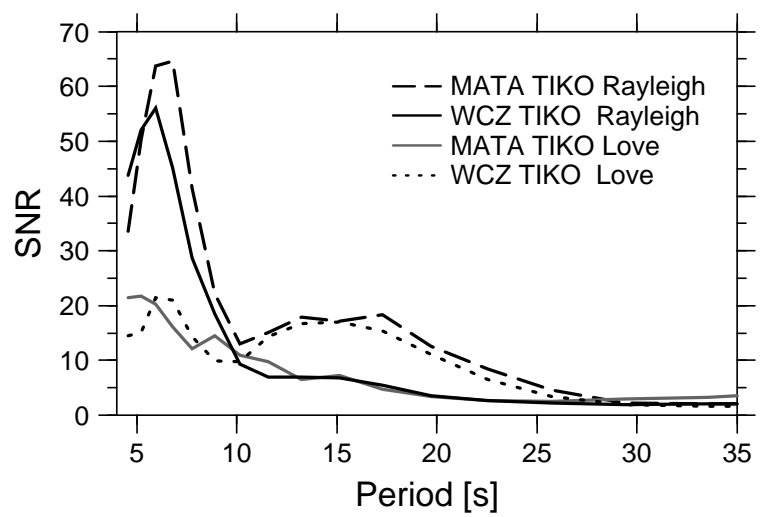

Figure 4.2 Signal-to-noise ratio (SNR) measurements for vertical (Rayleigh wave) and horizontal (Love wave) cross-correlations between stations TIKO-MATA and TIKO-WCZ (see Figure 4.1).
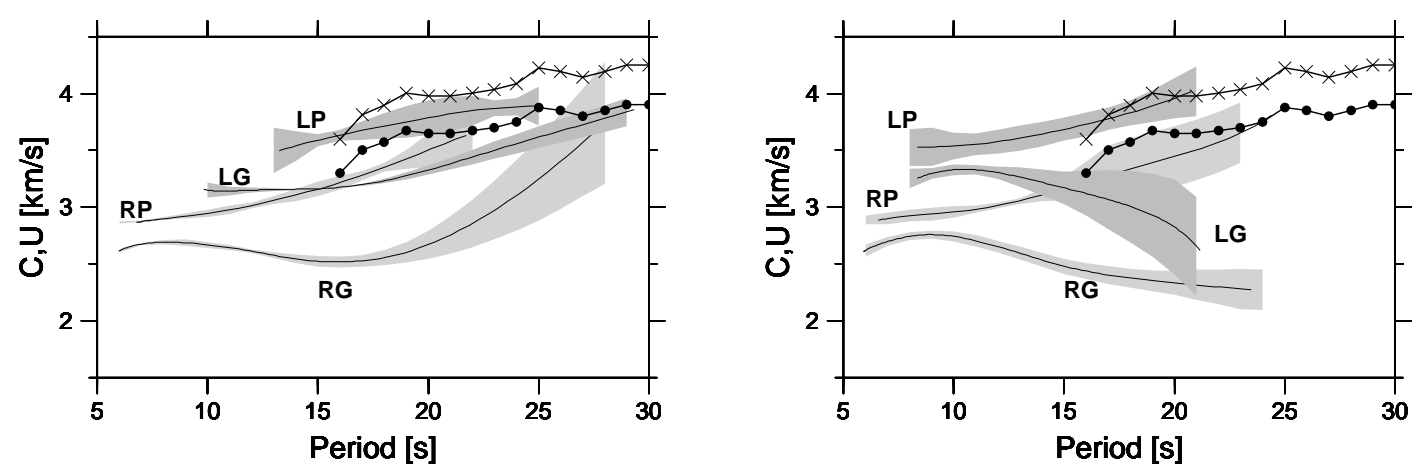

Figure 4.3 Left panel: Rayleigh wave group velocity (RG), Rayleigh wave phase velocity (RP), Love wave group velocity (LG) and Love wave phase velocity (LP) dispersion curve measurements for cross-correlations between TIKO and WCZ (solid lines). Gray shaded areas mark one standard deviation for each measurement point. The line marked by circles corresponds to the reference curve from Horspool et al. [2006] used for the Rayleigh phase velocity measurements. The line marked with crosses corresponds to the reference curve for Love wave phase veloctiy measurements. Right panel: same as the left panel for cross-correlations between TIKO and MATA (solid lines). 
velocity measurement, $x_{\text {calc }}$ the corresponding calculated value and $n_{F}$ is the total number of measurement points on a dispersion curve [Wathelet, 2005]. The grey shaded areas in Figure 4.4 and 4.5 illustrate the ranges in the shear-velocity-depth plane that are sampled by the NA. The colour depicts the fraction of the models with a misfit smaller than 0.1 that intersect a certain cell in the velocity-depth plane. The redder the colour, the more velocity profiles cross that portion of the velocity-depth plane. The black line indicates the weighted average of the models plotted in colour and the white line marks the average model derived by Horspool et al. [2006].

Inversion results for Rayleigh-wave phase and group velocities between TIKO and MATA (Figure 4.4 left panels) show three prominent velocity jumps, one at around $10 \mathrm{~km}$ depth, a small one around $20 \mathrm{~km}$ depth and the Moho at around $28 \mathrm{~km}$ depth. The top panels show the synthetic dispersion curves for 100 models randomly drawn from all models with a misfit smaller than 0.1. They are tightly distributed around the measurements (black dots). In contrast, the synthetic dispersion curves for the Rayleigh wave phase velocity between TIKO and WCZ are more spread out, reflecting the broad range of models with misfits smaller than 0.1 that we see in Figure 4.4 on the right bottom panel. For the reasons discussed above, we have not included the group velocity measurements between TIKO and $\mathrm{WCZ}$ in the inversion, which results in decreased resolution in this case relative to TIKO-MATA. However the average velocity-depth profiles (black lines) agree well for the two different station pairs down to a depth of about $22 \mathrm{~km}$, below which the dispersion curve for TIKO-WCZ loses sensitivity due to its truncation at $23 \mathrm{~s}$ period. In this context it should be mentioned that by inverting for 1-D profiles, we encounter a tradeoff between the maximum depth that can be imaged and horizontal resolution along an interstation path. In other words, the longer the interstation path-lengths, the less accurate any 1-D representation of a 3-D medium. Moreover shorter interstation distances result in shorter usable period ranges and correspondingly reduced depth resolution.

The histogram of the Moho depth - that is, the sum of crustal layer thicknesses - for the Rayleigh wave inversion between TIKO and MATA (Figure 4.6, dashed line) yields a mean Moho depth of $28.3 \mathrm{~km}$ and a standard deviation of $2.2 \mathrm{~km}$. This value corresponds within error margins to the depth of $25 \pm 2 \mathrm{~km}$ determined by Stern et al. [1987] from analysis of active-source seismic refraction data and to the $26 \pm 1 \mathrm{~km}$ to $29 \pm 1 \mathrm{~km}$ inferred by Horspool et al. [2006] from the joint inversion of teleseismic surface waves and receiver functions. The shear-velocity profiles calculated by Horspool et al. [2006] averaged for OUZ, WCZ and MATA (white line in Figure 4.4) show slightly higher velocities in the crust than obtained from the noise correlation but generally correspond well to the average model for depths greater than $\sim 4 \mathrm{~km}$. Our dispersion curves are computed 

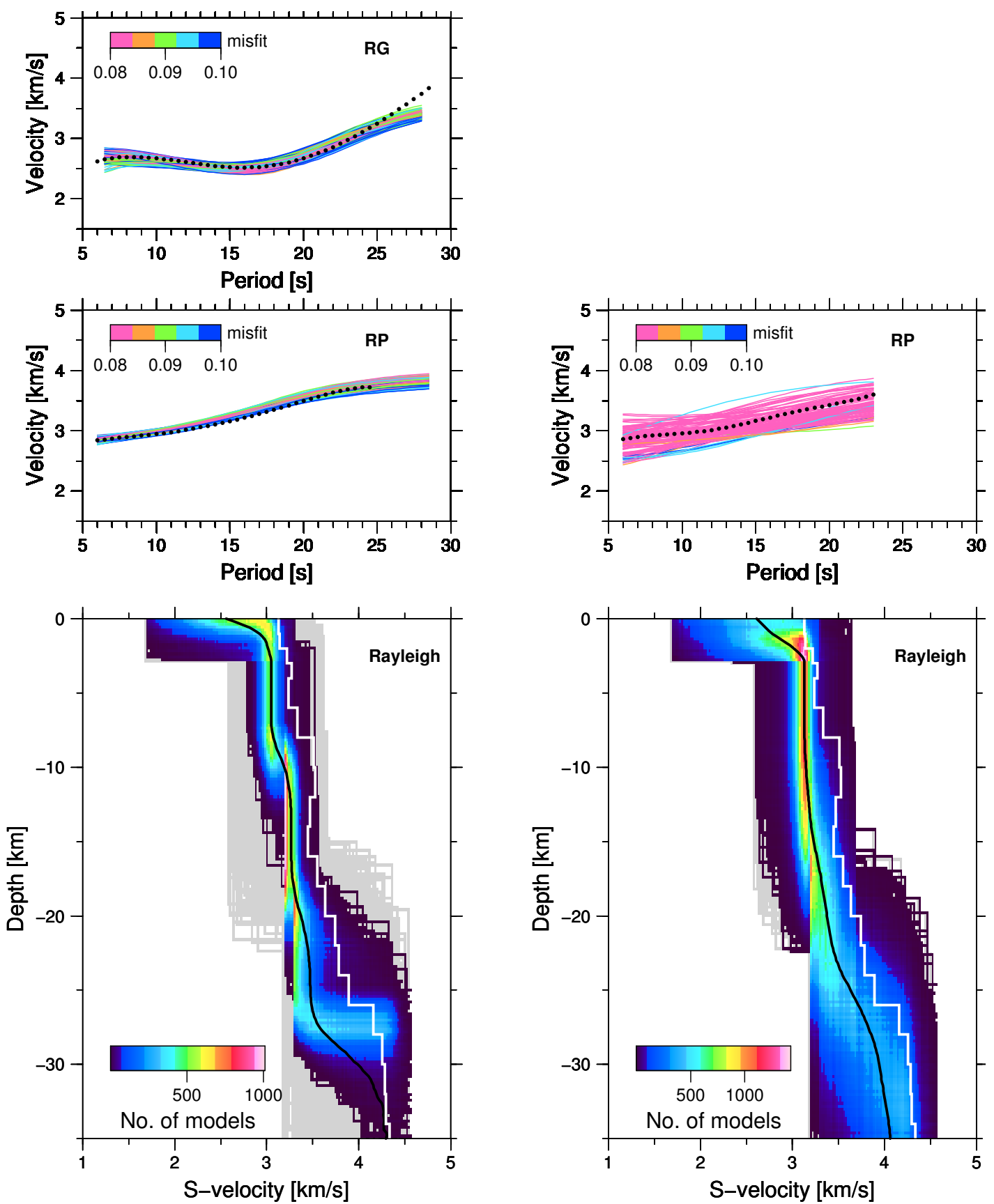

Figure 4.4 Inversion results for Rayleigh wave group and phase velocity dispersion curves measured between Tikorangi (TIKO) and Matakana (MATA; bottom left panel) and Rayleigh wave phase velocity dispersion curves between Tikorangi and Waipu Caves (WCZ; bottom right panel). The gray areas show the ensemble of all 10050 models evaluated, and the colors illustrate the density distributions of models with misfits smaller than 0.1. The thick black line in each case represents the weighted average of the models plotted in color. The white line shows the average model for the results of Horspool et al. [2006]. The top panels show the measured Rayleigh group (RG) and Rayleigh phase (RP) dispersion curves (black dots) on top of 100 synthetic dispersion curves that were drawn randomly from the corresponding ensemble of models with misfits smaller than 0.1 . 

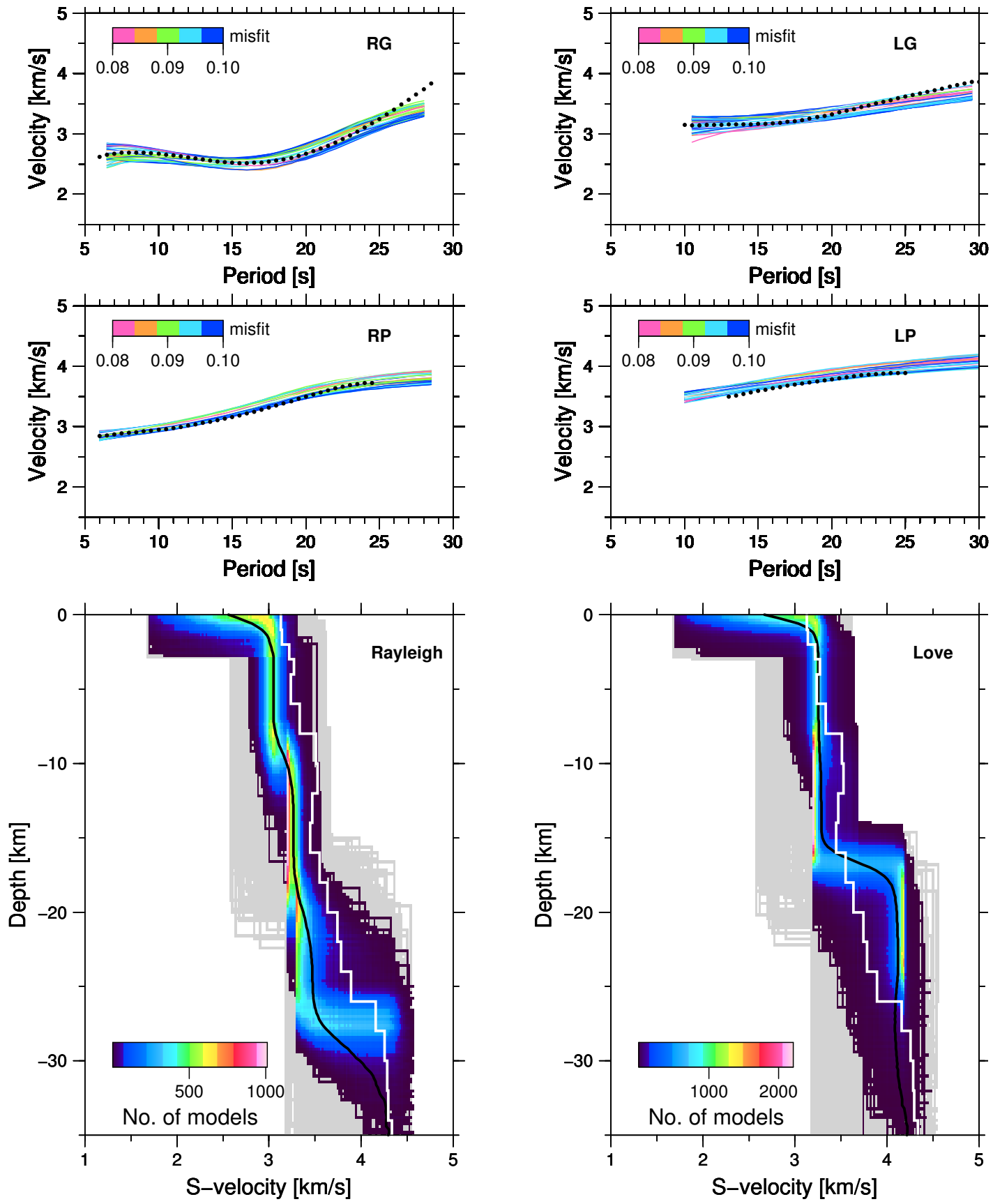

Figure 4.5 Inversion results for Rayleigh (bottom left panel) and Love wave (bottom right panel) group and phase velocity dispersion curves measured between Tikorangi and Matakana. The gray areas show the ensemble of all 10050 models evaluated, and the colors illustrate the density distributions of models with misfits smaller than 0.1 . The thick black line in each case represents the weighted average of the models plotted in color. The white line shows the average model for the results of Horspool et al. [2006]. The top panels show the measured Rayleigh group (RG) and Rayleigh phase (RP) dispersion curves (black dots, two top left panels) and Love group (LG) and Love phase (LP) dispersion curves (black circles, two top right panels) on top of 100 synthetic dispersion curves that were drawn randomly from the corresponding ensemble of models with misfit smaller than 0.1 . 


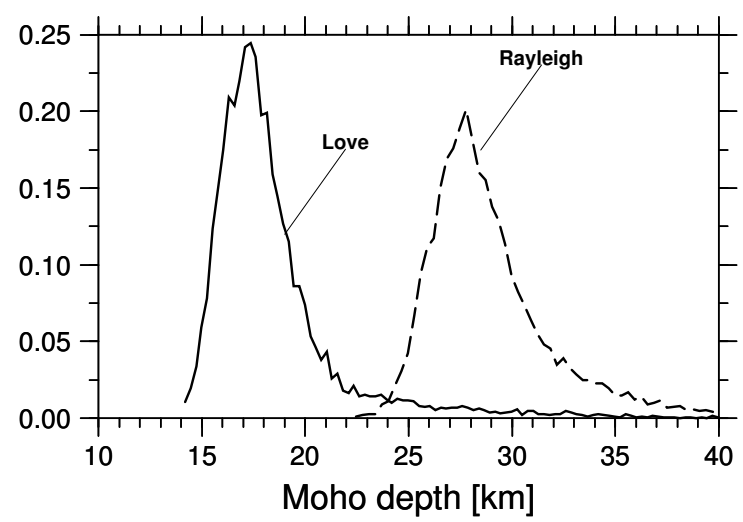

Figure 4.6 Normalized histograms of the Moho depth for the ensembles shown in Figure 4.5 in gray. The solid line indicates the histogram for the joint inversion of Love wave phase and group velocity measurements (right panels in Figure 4.5); the dashed line represents the histogram for the joint inversion of Rayleigh wave phase and group velocity (left panel in Figure 4.5).

for periods longer than 5 and $10 \mathrm{~s}$, as opposed to 15 and $20 \mathrm{~s}$ in Horspool et al.'s [2006] study, so our results are expected to better constrain the velocity structure at such shallow depths.

Compared to the Rayleigh wave inversion results (Figure 4.5 left panels), inversion of Love wave phase and group velocities between TIKO and MATA (Figure 4.5 right panels) show slightly higher velocities in the upper and middle crust and a Moho with a mean depth approximately $10 \mathrm{~km}$ shallower than observed with the Rayleigh wave data (Figure 4.6 solid line). One explanation for this discrepancy in Moho depths is a tradeoff between the thickness of the upper mantle layer and velocity in the lower crust layer. In other words, a velocity-depth model with a greater Moho depth requires higher velocities in the lower crust to fit the Love wave dispersion curves. With Love waves being sensitive mainly to horizontally polarised shear-waves, this discrepancy would be a possible indication of radial anisotropy in the lower crust. To test this hypothesis, we invert both Rayleigh and Love wave phase and group velocities between TIKO and MATA with the upper crust thickness fixed to $9 \mathrm{~km}$ and the Moho depth fixed at $27 \mathrm{~km}$. The results (Figure 4.7) show that both Love and Rayleigh waves can be fitted well for a common structural model by allowing considerably higher velocities for Love waves than for Rayleigh waves in the lower crust. We estimate the distribution of radial anisotropy by randomly drawing 1000 values from the S-velocity distribution for each layer and computing the radial anisotropy, which we here represent in terms of the magnitude of anisotropy $k=\left(V_{\max }-V_{\min }\right) / \bar{V}=2\left(V_{\mathrm{SH}}-V_{\mathrm{SV}}\right) /\left(V_{\mathrm{SH}}+V_{\mathrm{SV}}\right)$ [e.g., Stein and Wysession, $2003, \S 3.6 .2]$. For the upper and lower crustal layer we obtain values of $2 \pm 0.6 \%$ and 
$15 \pm 0.5 \%$ respectively. The uppermost mantle shows negative anisotropy of $6 \pm 0.5 \%$. However as the dispersion curves have little sensitivity to velocities at depths deeper than $\sim 30 \mathrm{~km}$, this deepest value may represent an artefact.

\subsection{Discussion and conclusion}

The results obtained by shear-velocity modelling of Rayleigh and Love wave dispersion curves derived from ambient noise correlation are in good agreement with those of previous studies using more traditional seismological techniques [Horspool et al., 2006; Stern et al., 1987]. This demonstrates that ambient noise correlation yields useful measurements even when the distributions of noise sources and seismographs are both highly asymmetric. Larose et al. [2006] demonstrated using 2-D numerical simulations that when the noise source distribution is asymmetric, a heterogeneous medium $(\sim 1.5$ scatterers/unit area) is required to enable reconstruction of the complete Green's function. If that condition is not met, Larose et al.'s [2006] analysis suggests that only those interstation paths lying parallel to the predominant source back-azimuth contribute to an estimate of the causal part of the Green's function in the cross-correlation process. The NORD deployment's linear geometry means that the interstation paths are uniformly parallel and oriented northwest-southeast. Sinclair [2002] observed the highest density of tropical cyclones in the southwest Pacific from 1970 until 1997 to the northwest of New Zealand. In other words, the interstation paths considered here are sub-parallel to the direction in which most noise is generated. This is in agreement with noise source backazimuths estimated by Lin et al. [2007, Figure 15] from differences in signal-to-noise ratios between the causal and anticausal part of the cross-correlations. The results of beamforming measurements using a 61-element array in the western North Island [Brooks et al., 2009a, b] also show the northwest of the North Island as one of the dominant ambient noise sources in New Zealand.

Our analysis suggests that radial shear-velocity anisotropy is required to account for differences in the Rayleigh and Love group and phase velocity dispersion curves, which depend on vertically- and horizontally-polarised shear-velocities, respectively [see Endrun et al., 2008, for a recent discussion]. We estimate the magnitude of such radial anisotropy for the TIKO-MATA path to be $\sim 2 \%$ in the upper crust and $\sim 15 \%$ in the lower crust. The comparison of the Love wave inversion results illustrated in Figure 4.5 and Figure 4.7 shows that the Love wave dispersion curves between TIKO and MATA can be equally well fit with two distinctly different groups of shear-velocity models. Two possible explanations for this are that there is either no single global minimum within the 

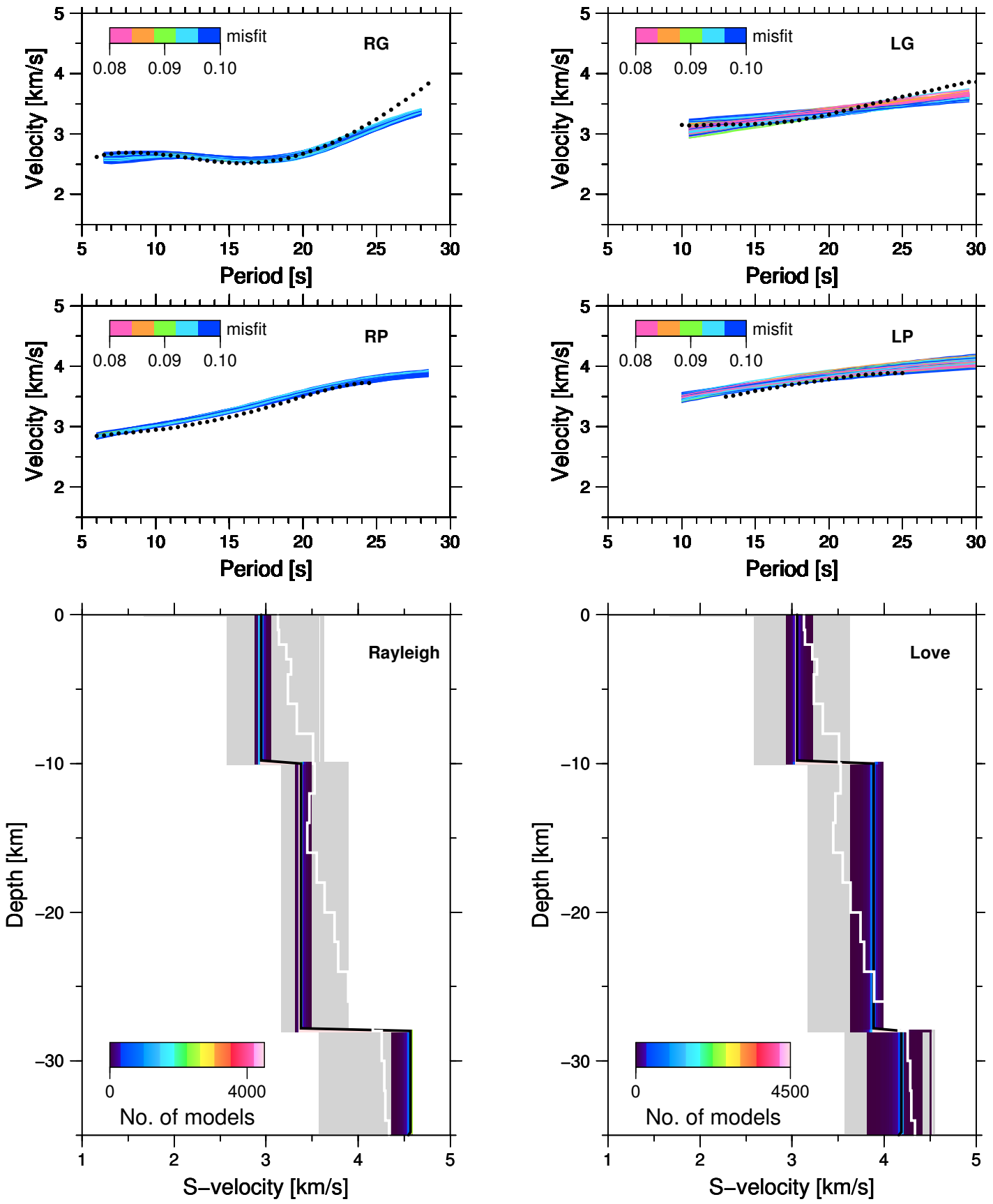

Figure 4.7 Inversion results for Rayleigh (bottom left panel) and Love wave (bottom right panel) group and phase velocity dispersion curves measured between Tikorangi and Matakana using fixed layer thicknesses. The gray areas show the ensemble of all 10050 models evaluated, and the colors illustrate the density distributions of models with misfit better than 0.1 . The white line shows the average model for the results of Horspool et al. [2006]. The top panels show the measured Rayleigh group (RG) and Rayleigh phase (RP) dispersion curves (black circles, two top left panels) and Love group (LG) and Love phase (LP) dispersion curves (black dots, two top right panels) on top of 100 synthetic dispersion curves that were drawn randomly from the corresponding ensemble of models with misfits smaller than 0.1 . 

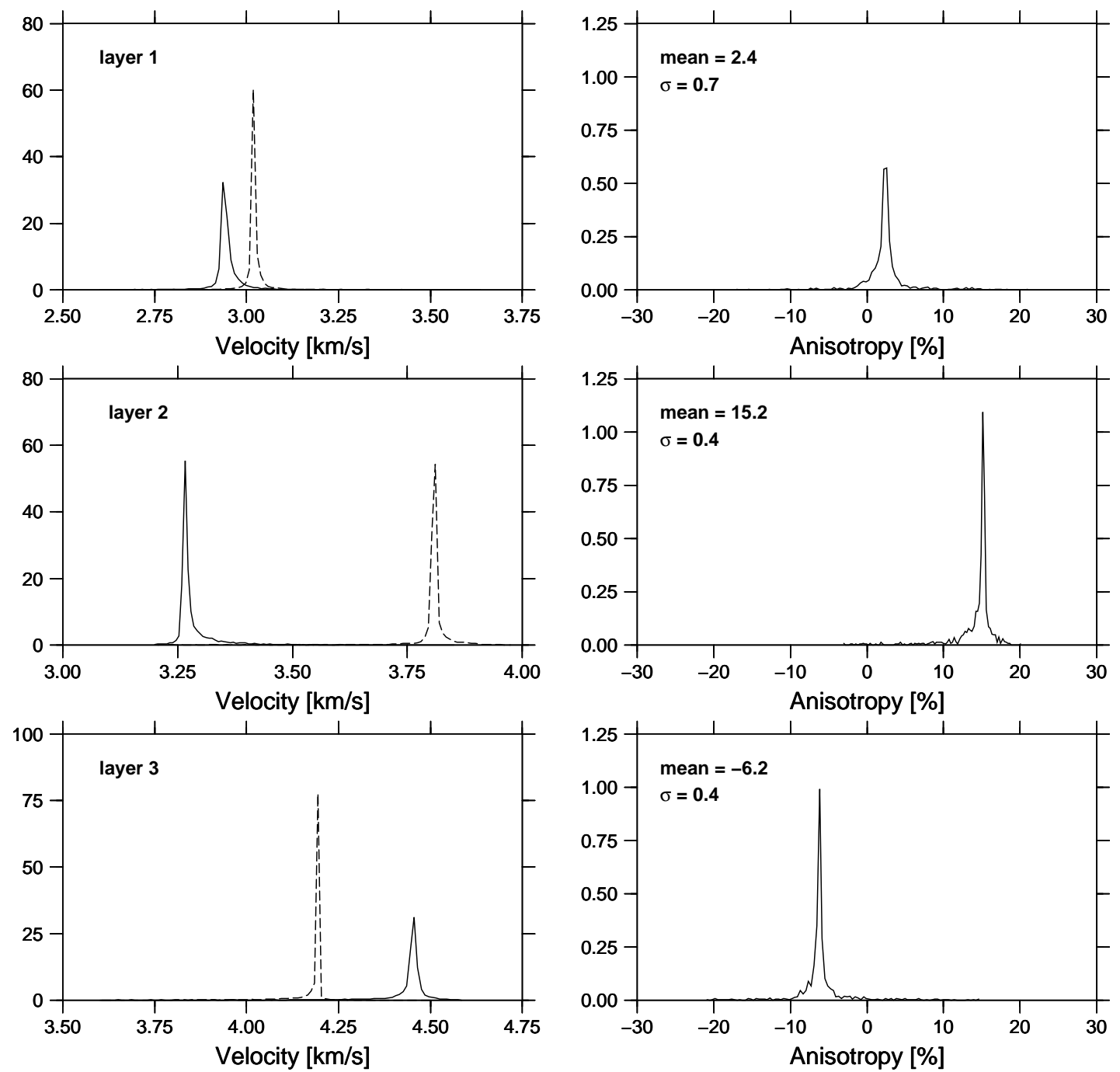

Figure 4.8 Estimates of radial anisotropy in the upper (layer 1) and lower crust (layer 2) and the uppermost mantle (layer 3). The lefthand panels show histograms for S-velocity calculated from the Rayleigh wave inversion ensemble (dashed lines) and Love wave inversion ensemble (solid lines) shown in Figure 4.7. The righthand panels show the histograms for radial anisotropy, calculated by randomly drawing 1000 values from the corresponding lefthand panels. 
given parameter space or that a local minimum exists that is close to the global minimum due to the tradeoff between lower crustal and upper mantle velocities. The latter problem stems from the poor depth resolution of surface waves and may be ameliorated by including further a priori constraints in the inversion. The estimates of $\sim 15 \%$ of radial anisotropy in the lower crust and $\sim-6 \%$ in the uppermost mantle are also likely to be influenced by the tradeoff between lower crustal and upper mantle velocities. Moschetti et al. [2010] reported a negative correlation between radial anisotropy inferred from surface waves in the upper mantle and the lower crust beneath the western US. To overcome this problem would require the inclusion of anisotropy in the inversion as an additional parameter or set of parameters which is beyond the scope of this study. The estimates of radial anisotropy presented here should therefore be treated as indicative of strong radial anisotropy either in the crust or the uppermost mantle.

Aside from near-radial anisotropy of 4-6\% inferred from receiver function analysis and interpreted to be caused by schists derived from subducting sediments above the Pacific plate [Savage et al., 2007], we are not aware of other estimates of radial anisotropy in the upper or lower crust in greater New Zealand although azimuthal variations in anisotropy have been extensively studied [e.g. Marson-Pidgeon et al., 1999; Gerst and Savage, 2004; Audoine et al., 2004; Balfour et al., 2005; Duclos, 2005]. Using Love and Rayleigh waves from teleseismic events, Endrun et al. [2008] inferred radial anisotropy values for the lower crust in the Aegean region of between 4.0 and $7.7 \%$, with a maximum of $15 \%$. Shapiro et al. [2004] used surface wave dispersion measurements from teleseismic earthquakes to study crustal radial anisotropy in Tibet and related this to crustal thinning and mid-to-lower crustal flow. They observed values of around $8 \%$ in the lower crust. Azimuthal anisotropy as well as multipathing may contribute to traveltime differences between Love and Rayleigh waves [e.g. Endrun et al., 2008]. Yao and Van Der Hilst [2009] inferred a maxium phase velocity bias of $3 \%$ from simulations of a heterogenous noise source distribution surrounding a homogeneous medium, which would correspond to variations of $\sim 0.1 \mathrm{~km} / \mathrm{s}$ in our phase velocity measurements. Variations in P-velocity can also affect the Rayleigh wave dispersion curves but not the Love wave dispersion curves, and thereby influence the estimated magnitude of radial anisotropy. Wathelet [2005] showed in sensitivity tests for a two-layer model that for Poisson's ratios of larger than $\sim 0.27$, the influence of the P-velocity on Rayleigh wave dispersion curves is negligible. While the a priori values of 0.26 in the crust and 0.28 in the mantle assumed by Horspool et al. [2006] lie within a range that might still affect the dispersion curves, our observed values of $\sim 0.31$ in the crust and $\sim 0.35$ in the upper mantle are unlikely to influence the $S$ velocity profiles inferred from Rayleigh wave dispersion curves. These points should be taken into account in future studies of radial anisotropy in New Zealand and elsewhere. 
The $\sim 28 \mathrm{~km}$ crustal thickness inferred here for the Northland Peninsula is similar to values observed throughout the western North Island of New Zealand [Horspool et al., 2006; Stern et al., 1987; Stratford and Stern, 2006]. Such values are approximately 10 $\mathrm{km}$ less than the global average thickness of continental crust [e.g. Fowler, 2005] and 7-12 km less than observed in the southern North Island [Savage, 1998; Holt and Stern, 1994].

Stern et al. [1987] demonstrated that a $25 \mathrm{~km}$-thick crust would require a hotter than normal mantle lid to generate sufficient buoyancy to lift the Northland Peninsula above sealevel. Limited crustal heat flow measurements suggest that the crust is somewhat hotter than normal in Northland [74 $\pm 4 \mathrm{~mW} \mathrm{~m}^{-2}$; Pandey, 1981], although heat flow studies conducted elsewhere in New Zealand suggest that those measurements are biased towards higher values by $10-15 \mathrm{~mW} \mathrm{~m}^{-2}$ due to assumptions about thermal conductivity [Funnell et al., 1996; Townend, 1999; Goutorbe et al., 2008]. As noted above, P velocities in the uppermost mantle beneath Northland are relatively low [i.e. $7.6 \mathrm{~km} \mathrm{~s}^{-1}$ at $25 \mathrm{~km}$; Stern et al., 1987], and similarly low values are seen further south in the western North Island in active source [Stratford and Stern, 2006] and mantle refraction (Pn) studies [Seward et al., 2009].

Stern [2006] recently hypothesised that the crust beneath the western North Island, including the Northland Peninsula, has been thinned by the convective removal of part of the lower crust and lithospheric mantle in response to a Rayleigh-Taylor instability. According to this model, upper-plate lithosphere is thickened during subduction to such an extent that the thickened mantle root and lower crust become gravitationally unstable and detach from the upper crust, thereby thinning the crust and allowing hot asthenospheric material to upwell beneath it. As noted in the introduction, Northland is thought to have been part of a subduction zone at approximately $25 \mathrm{Ma}$. If the Rayleigh-Taylor model is appropriate for this region, then future heat flow measurements may substantiate the seismological evidence for hot mantle material at depths of $\sim 25 \mathrm{~km}$.

In conclusion, we have demonstrated in this chapter the suitability of ambient noise correlation measurements for shear wave velocity modelling for a non-optimal network configuration (namely, a linear array of five stations operated for up to 14 months) and highly directional noise-source distributions. The 1-D shear-velocity models computed from surface wave dispersion curves obtained from vertical component ambient noise correlations show good agreement with the results of earlier studies based on active source experiments and teleseismic earthquakes. In particular, the inversion of Rayleigh wave phase and group velocity dispersion curves yields a Moho depth of $\sim 28 \mathrm{~km}$, in close agreement with the estimates of Stern et al. [1987] and Horspool et al. [2006]. We have extended 
previous studies of the Northland Peninsula by analysing horizontal component records, which have allowed us to estimate values for radial anisotropy in the crust of $2-15 \%$ by comparing the shear-velocity profiles inferred from Love and Rayleigh wave dispersion curves.

In Chapter 5, we apply similar preprocessing and cross-correlation techniques to a larger dataset including recordings from four temporary deployments and permanent seismic stations on the North Island and extend the shear-wave velocity modelling into three dimensions. 
4. S-velocity structure of the Northland Peninsula 


\section{Ambient noise tomography of the central North Island using temporary and permanent seismograph data ${ }^{1}$}

The central North Island of New Zealand is characterized by extension, high heat flow and active volcanism caused by the Pacific plate subducting beneath the Australian plate off the east coast of the North Island. The Taupo Volcanic Zone (TVZ) is the current locus of rifting and active volcanism. The shear velocity structure derived here from short-period surface wave analysis fills a gap in the geophysical information obtained in previous active and passive source studies. Using ambient noise correlation techniques, we reprocess data from four three-component temporary seismic arrays that were deployed between 2001 and 2005 in the central North Island, and data from permanent seismic stations operational at the same time. Special attention has been paid to the correction of timing errors and incomplete instrument response information. Low shear velocities in the top 15-20 km of the crust inferred from Rayleigh and Love wave dispersion analysis coincide with the presumed source regions of rhyolitic magma in the Taupo Volcanic Zone and are correlated with conductive bodies inferred from a 3D magnetotelluric survey. Based on the observed changes in shear velocity we use empirical relations to infer an average percentage of partial melt in the upper crust of $<4.2 \%$. Comparison of our shear velocity model with compressional wave cross-sections from active and passive source studies and shear velocities derived from previous $3 \mathrm{D} \mathrm{Vp}$ and $\mathrm{Vp} / \mathrm{Vs}$ models reveals several consistent features, especially in the lower crust. The greater sensitivity of shear velocity than compressional velocity to the presence of melt and other fluids aids in linking seismic and electromagnetic observations and provides additional constraints on the distribution of magmatic structures in active continental rifting environments.

\footnotetext{
${ }^{1}$ Parts of this chapter previously published by Behr et al., Geochem., Geophys., Geosys., 2011
} 


\subsection{Introduction}

The TVZ is a young volcanic arc, similar in size and structural complexity to the Yellowstone volcanic field. It has produced exceptionally large volumes of rhyolitic magma (at least 10,000 $\mathrm{km}^{3}$ ) during the last 1.6 Myr [Rowland et al., 2010; Wilson et al., 1995] which makes it the most frequently active and productive silicic volcanic system on Earth. Rhyolitic volcanism is located in the centre of the TVZ [Wilson et al., 1995] with Taupo and the Okataina volcanoes marking the southern and northern extents of the central TVZ (Figure 5.1). Andesitic and basaltic volcanism has also occurred but at much lower volumes than the rhyolitic volcanism, and with the eruptive products mainly confined to the northern and southern ends of the TVZ. Prominent examples of andesite composite volcanoes are Mt. Ruapehu at the southern end of the TVZ and White Island in the Bay of Plenty (Figure 5.1).

Convergence rates of the Pacific plate towards the Australian plate underneath the North Island decrease towards the south. This is proposed to result in a clockwise rotation of the eastern North Island relative to the Pacific and Australian plates to explain GPS measurements showing the largest extension rate in the Bay of Plenty (Figure 5.1) at the northern end of the TVZ and converting into contraction to the south of the TVZ [Wallace, 2004]. Rotation of the eastern side of the North Island relative to the western side is also put forward to explain rotation of sediments on the eastern North Island inferred from paleomagnetic data [Walcott, 1984; Lamb, 1988]. Wilson et al. [1995] pointed out that the lack of rotation observed in pre-Quaternary sediments on the Bay of Plenty coast, east and west of the rifted zone, contradict the concept of a rotational opening of the TVZ and that the increase of extension rates from south to north as observed by GPS [Wallace, 2004] might describe the elastic but not the inelastic deformation of the crust. The onset of rifting in the TVZ is inferred to be approximately 0.9 Ma by Wilson et al. [1995] from the observable tilting and faulting of volcanic deposits, whereas Stern et al. [1987] proposed that the opening started at $\sim 4$ Ma based on the apparent migration of the active volcanic front and paleomagnetic data.

The tectonic evolution of the lithosphere beneath the TVZ is a matter of ongoing debate. Pulford and Stern [2004], Stratford and Stern [2006], Stern et al. [2006] and Stern and Benson [2011] proposed crustal thinning beneath the central and western North Island due to delamination of lower crust and upper mantle prior and coeval to the onset of rifting in the TVZ. This was followed by mafic underplating of the crust from a now hot and buoyant upper mantle. An alternative proposed mechanism is to accommodate extension in the lower crustal lithosphere beneath the central TVZ mainly by mafic 
dyke intrusions from the mantle into a crust of constant thickness [Rowland et al., 2010; Harrison and White, 2006]. These alternative mechanisms have different implications on the observed tectonic uplift of the central North Island as discussed in more detail in Section 5.6. For a recent review of these models see, for example, Rowland et al. [2010].

Information pertaining to the crustal S-velocity structure of the TVZ may help address some of the outstanding questions regarding its evolution, as S-waves are sensitive to slightly different physical properties than P-waves. In particular, S-wave velocities depend more strongly on the shear modulus than P-waves and are therefore more sensitive to the presence of fluids and small proportions of partial melt in a material [Stein and Wysession, 2003].

S-velocity structure has been generally inferred using three-component seismometers and earthquake sources [e.g. Reyners et al., 2006; Sherburn et al., 2006]. As three-component seismographs are required to identify S-wave arrivals, which are in any case more difficult to detect than P-wave arrivals, the number of identified P-wave arrivals always exceeds the number of S-wave arrivals. This results in different resolutions for S-wave and Pwave models and makes their joint interpretation difficult [Eberhart-Phillips, 1990]. It is therefore quite common to invert for the ratio of $\mathrm{P}$ - to $\mathrm{S}$-velocity $(\mathrm{Vp} / \mathrm{Vs})$ rather than for a true S-velocity model [Eberhart-Phillips et al., 2005; Reyners et al., 2006], based on the assumption that it is more physically reasonable to assign constant $\mathrm{Vp} / \mathrm{Vs}$ values than constant Vs values to parts of the model where Vs is unconstrained by observations. Nevertheless, new inversion approaches incorporating double-difference algorithms can solve for $\mathrm{Vp}$, Vs and Vp/Vs simultaneously [e.g. Zhang et al., 2009]. An alternative method of inferring S-velocity structure from earthquake recordings is to utilize surface wave dispersion measurements [Brisbourne and Stuart, 1998]. However, as seismic waves experience stronger attenuation at higher frequencies, surface wave dispersion curves from teleseismic earthquakes seldom yield reliable results at periods shorter than $\sim 20 \mathrm{~s}$ and therefore have little sensitivity to crustal structures [Yao et al., 2006]. Inferring surface wave velocities from the cross-correlation of ambient seismic noise recordings fills this geophysical gap and is the focus of this chapter.

As discussed in Chapter 1, using ambient seismic noise as a pervasive energy source has become an established strand of seismology during the last few years [Shapiro and Campillo, 2004; Yang et al., 2007; Yao et al., 2006; Lin et al., 2008; Nishida et al., 2008; Liang and Langston, 2009]. It has been known since the 1950s that surface wave information can be extracted from ambient seismic noise using correlation techniques [Aki, 1957]. Applications followed in seismology [Claerbout, 1968], in helioseismology [Rickett and Claerbout, 2000] and in acoustics [Lobkis and Weaver, 2001], 
but it is only in the last decade that this method has received widespread attention within the broader seismological community. Ambient seismic noise tomography requires only short instrument deployment times and no artificial sources, thereby providing an economical and low-impact way of gaining new insights into crustal and upper mantle structure [Shapiro et al., 2005; Lin et al., 2007, 2008; Brenguier et al., 2007; Yang et al., 2007; Yao et al., 2006, 2008].

In this chapter, we measure Rayleigh and Love wave phase and group velocity dispersion curves from cross-correlation functions of ambient seismic noise recorded at stations of the Central North Island Passive Seismic Experiment [CNIPSE; 33 stations; 6 months Reyners and Stuart, 2002], the Western Central North Island Passive Seismic Experiment [WCNIPSE; 6 stations; 9-11 months Greve et al., 2008], the Seismic Tomography Around Ruapehu and Tongariro deployment [START; 28 stations; 5 months Rowlands et al., 2005], the RF2004 deployment [7 stations; 10 months Bannister et al., 2004] and three permanent broadband stations that started operating in 2001 (see Figure 5.1). We also include correlations between 20 permanent broadband stations operated by GeoNet (http://www.geonet.org.nz). We use the resulting dispersion information to compute crustal S-velocity structure for horizontally and vertically polarized S-waves to depths of $\sim 20$ and $\sim 40 \mathrm{~km}$, respectively, and assess the constraints these results impose on the lithology of the crust and uppermost mantle. This builds upon results of the broader ambient noise study of New Zealand by Lin et al. [2007], who used a smaller number of permanent stations only, and the small-scale analysis of the northwestern North Island by Behr et al. [2010].

\subsection{Noise source characteristics}

It has been shown in several studies that strongly anisotropic noise source distributions can bias the surface wave velocity measurements from noise cross-correlation functions [Tsai, 2009; Yao and Van Der Hilst, 2009]. Ambient noise at frequencies less than $1 \mathrm{~Hz}$ is mainly ocean-generated [Bonnefoy-Claudet et al., 2006; Longuet-Higgins, 1950] and its spectrum exhibits two characteristic peaks: one at approximately $14 \mathrm{~s}$ period (the primary microseism) and the other at $\sim 7 \mathrm{~s}$ period (the secondary microseism).

In order to describe the azimuthal distribution of noise sources represented in the noisecorrelations in the central North Island, we have employed plane wave beamforming [e.g. Brooks et al., 2009a] using noise recorded during 130 days of the START deployment (see Figure 5.1) on which ten or more stations were operating at the same time. Figure 


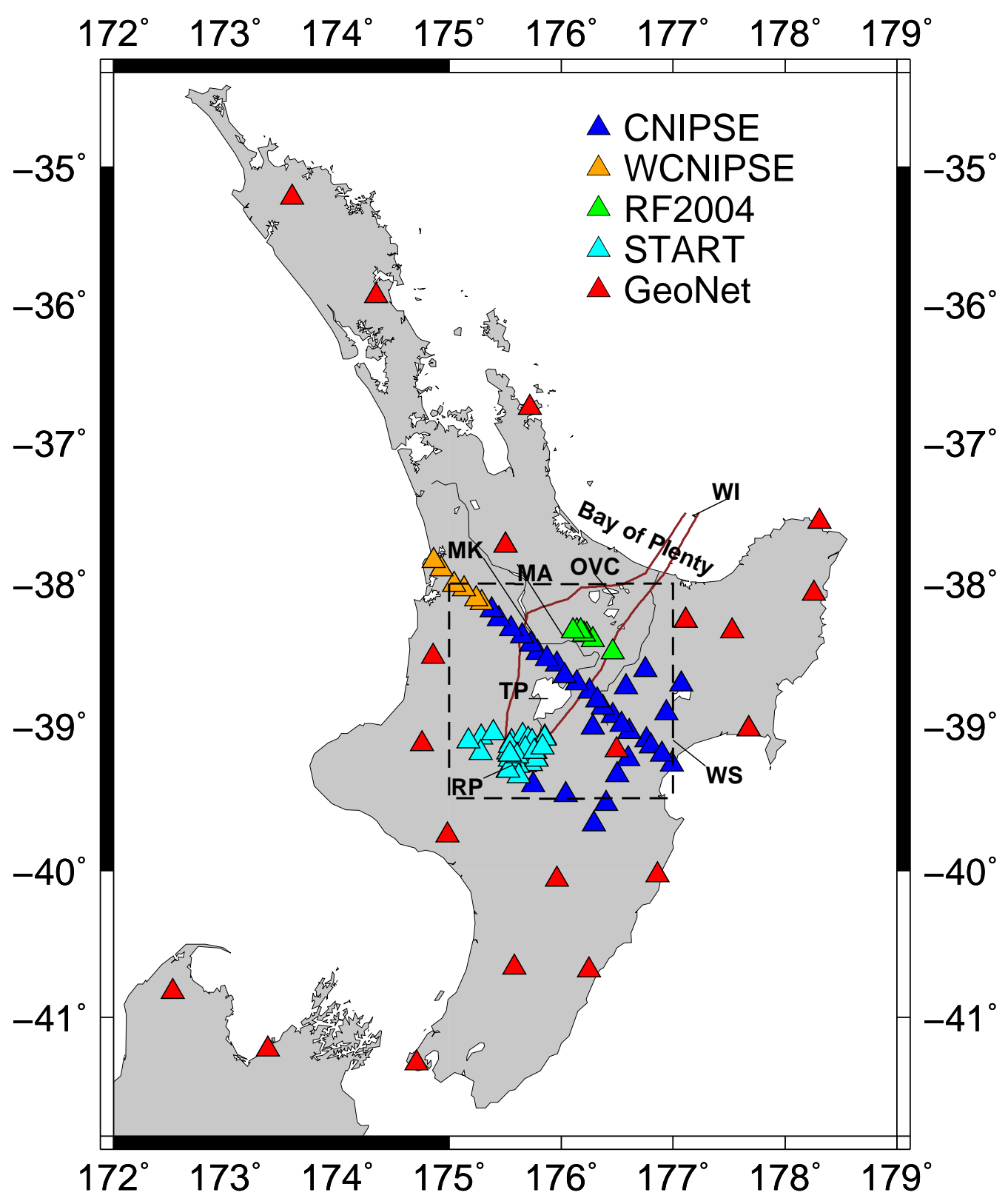

Figure 5.1 Stations in the Central North Island used in this study. Stations of the START and CNIPSE deployment as well as several GeoNet stations and stations of the WCNIPSE and RF2004 deployment were operating at the same time. Cross-correlations between the permanent GeoNet stations were added to extend the dataset above periods of $12 \mathrm{~s}$. The dashed line marks the area with highest path density which was the focus for the pseudo-3D inversion. The brown line describes the outline of the TVZ. The prominent rhyolitic volcanoes illustrated are Taupo (TP), the Okataina Volcanic Centre (OVC), Mangakino (MK) and Maroa (MA). Prominent andesite composite volcanoes are Mt. Ruapehu (RP) and White Island (WI). The Wairoa Syncline (WS) is a deep sedimentary basin on the East Coast. 
5.2 shows the array response, the beamforming result for a theoretical source below the array [for a more detailed discussion of array responses see Wathelet et al., 2008], at four periods spanning the primary microseism $(16.0 \mathrm{~s}$ and $12.8 \mathrm{~s})$ and secondary microseism $(8.0 \mathrm{~s}$ and $6.1 \mathrm{~s})$. As the apparent velocity for such a source is infinite, the response of a perfect array should therefore be a single point. The response is conceptually similar to what is referred to in other scientific fields, such as astrophysics, as the Point Spread Function (PSF) [Bertero and Boccacci, 2005]. The response for the START array at all periods analyzed here is an ellipse where the major axis is oriented SSW-NNE. This response results in larger velocity smearing for waves coming from southern or northern directions than for waves coming from the east or west. In contrast the azimuth for waves coming from eastern or western directions will be more smeared than for waves coming from the south or north. The array response also reveals that signals with periods close to that of the secondary microseism will be better resolved than signals with periods close to that of the primary microseism. Despite this imperfect response, the START deployment is nevertheless well-suited to our purpose, as it does not exhibit any secondary peaks in the array response within the velocity-period range of interest and therefore does not introduce any azimuthal ambiguity in the beamforming results.

On most of the examined 130 days, peaks in the beamforming results show striking correlation with ocean wave energy for the same day at the $50 \mathrm{~m}$ isobath around New Zealand's coast [taken from the NIWAM wave prediction model, supplied by the National Institute of Water and Atmospheric Research, NIWA, http://www.niwa.co.nz, see also Gorman et al., 2003a, b]. An example is shown in Figure 5.3 for 22 February 2001 at $6 \mathrm{~s}$ period. We return to the topic of interrelation between noise directionality and ocean wave energy in chapter 6.

Figure 5.4 shows the beamforming results for $6.1 \mathrm{~s}$ and $10 \mathrm{~s}$ periods averaged over all 130 days analyzed. The $6.1 \mathrm{~s}$ period results exhibit two maxima, one in the southwest of the START array and one in the southeast. Although different in amplitude, the noise sources are present at almost all azimuths other from the northeast. Waves coming from the northeast to the START array have to travel through the TVZ, which has been known for some time to be highly attenuative [e.g. Satake and Hashida, 1989]. Further evidence for the highly attenuative nature of the TVZ is provided by recent results obtained using the dense MORC array [A. Benson, personal communication, Stern and Benson, 2011]. Passive surface waves are visually apparent in data recorded between shots because the station spacing is below the Nyquist wavenumber for surface waves from ambient noise sources. The surface waves are coherent and have a constant apparent velocity to the east and west of the TVZ: within the TVZ, however, velocities appear to drop and coherence 

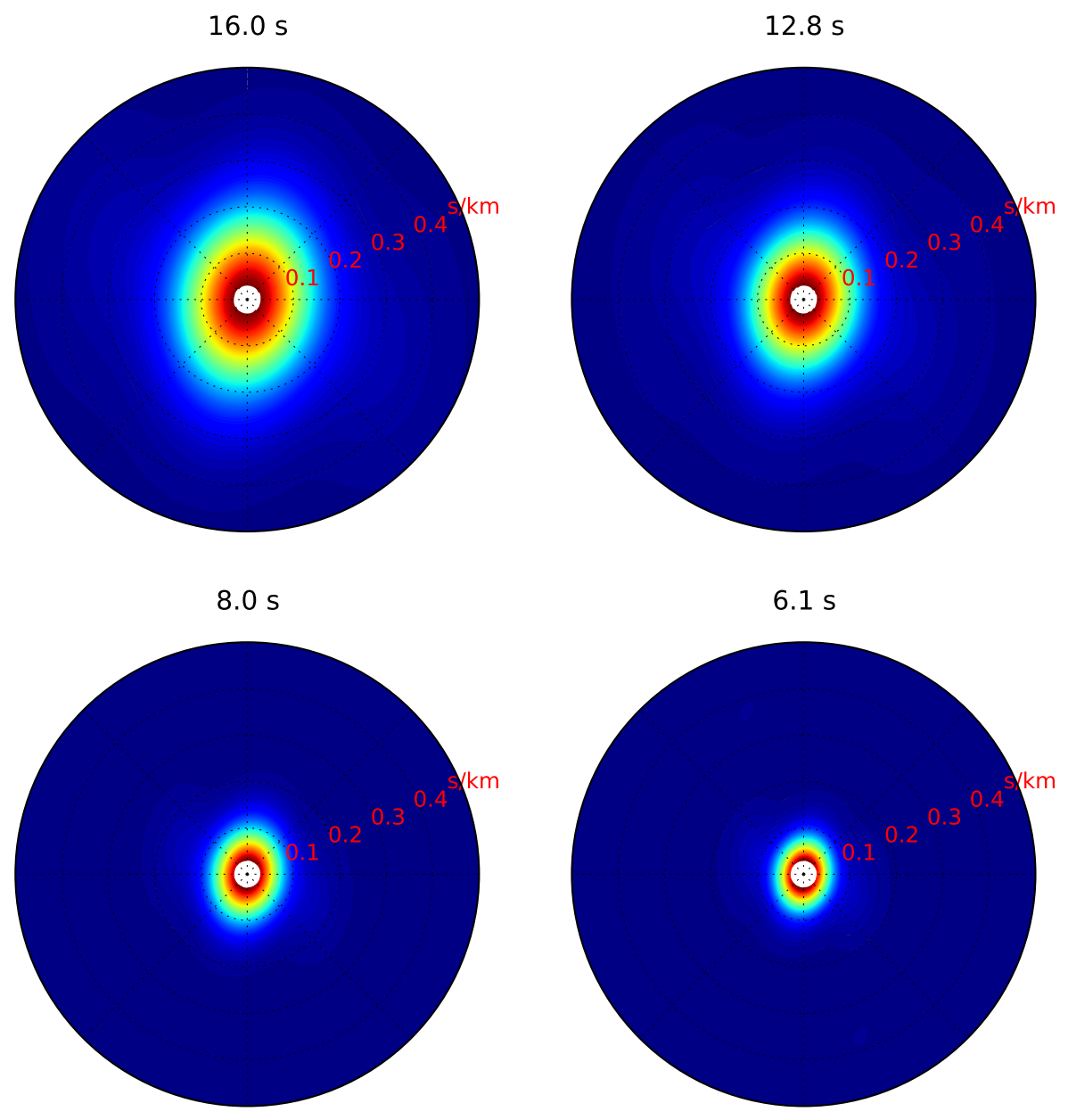

Figure 5.2 Array response for the START deployment at four periods close to the primary $(16.0 \mathrm{~s}$ and 12.8 s) and secondary microseisms (8.0 s and $6.1 \mathrm{~s})$. 

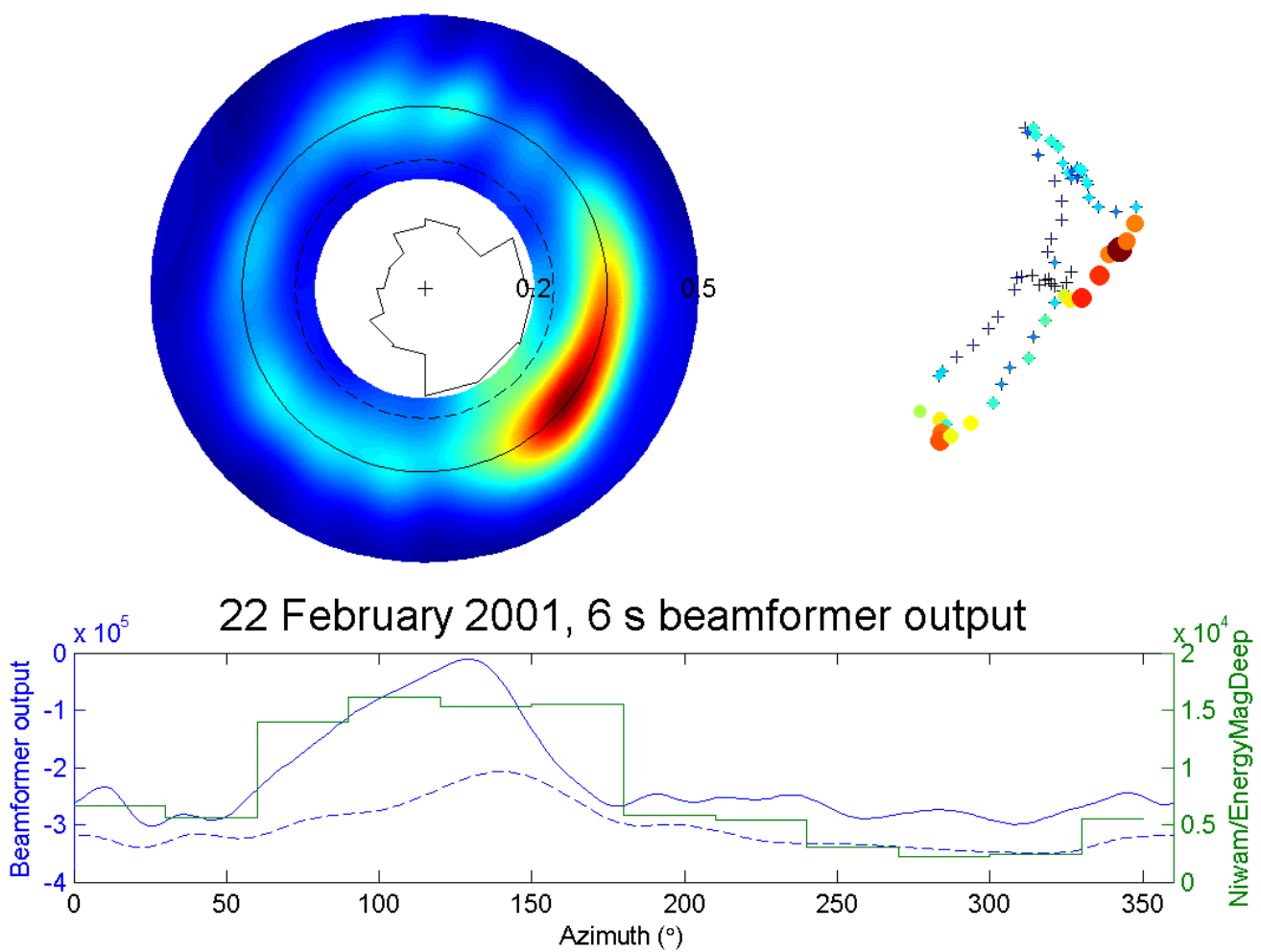

Figure 5.3 Plane wave beamforming results at $6 \mathrm{~s}$ period for ambient noise recorded on 22 February 2001 at 22 seismometers during the START deployment (see Figure 5.1). The upper left panel is the beamforming result with the ocean wave energy flux shown in the middle. The upper right panel shows the ocean wave energy at the $50 \mathrm{~m}$ isobath taken from the NIWAM model (supplied by the National Institute of Water and Atmospheric Research, NIWA, from their wave prediction model). The lower panel shows the beamformer amplitude as a function of azimuth taken at 0.3 and $0.2 \mathrm{~s} / \mathrm{m}$ slowness as solid blue and dashed blue line, respectively, and the azimuthal distribution of the ocean wave energy binned at $3^{\circ}$ intervals. 

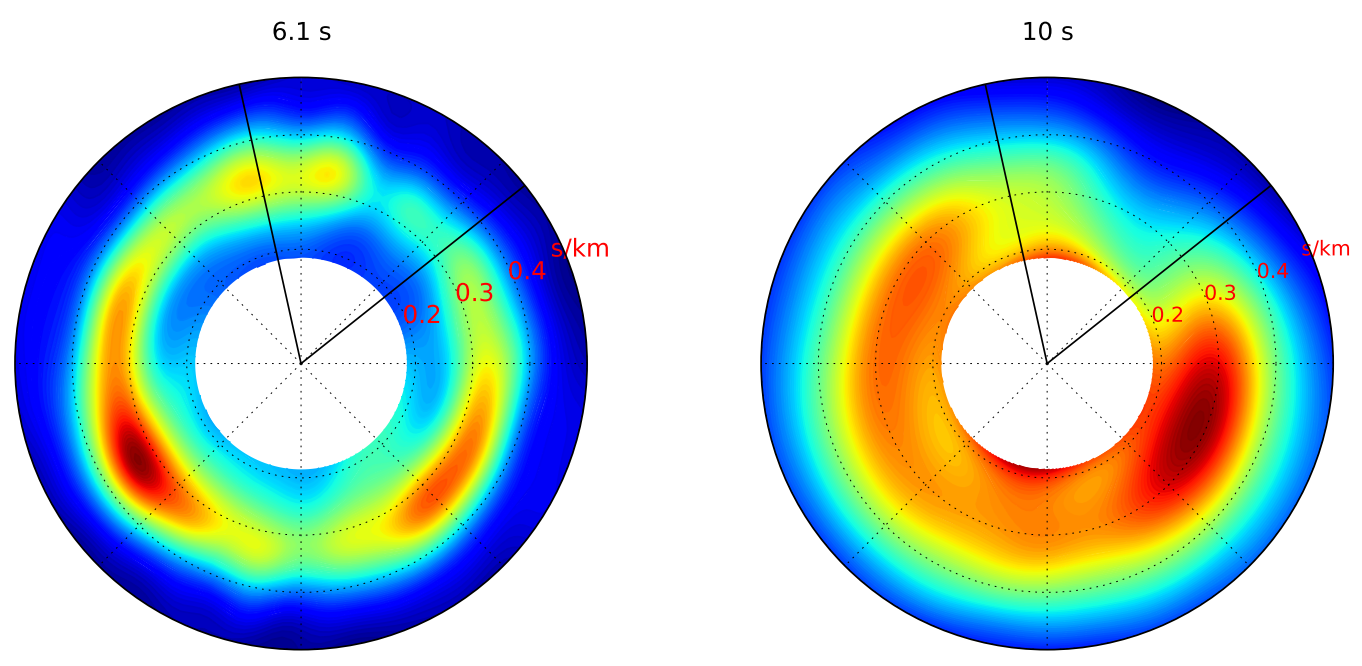

Figure 5.4 START beamforming results at the $6.1 \mathrm{~s}$ and $10 \mathrm{~s}$ periods averaged over 130 days of the deployment with the black lines marking the azimuthal extent of the TVZ in respect to the center of the START array.

decreases significantly. The same gap can be observed in our beamforming results for the $10 \mathrm{~s}$ period. The velocity distribution at this period is more diffuse than for the $6.1 \mathrm{~s}$ period, which reflects the broader array response for this period (see Figure 5.2).

Because the noise source distribution, revealed by the START beamforming results, varies quite smoothly, we assume the noise field to be azimuthally homogeneous to first-order. Yao and Van Der Hilst [2009] showed that phase velocity bias can be up to $3 \%$ for a rapidly varying azimuthal noise source distribution but is less than $1 \%$ for a smooth variation. The results also show that fully modelling the cross-correlation functions would not only require us to take the azimuthal dependence of noise sources into account but also the wave travel path and attenuation. We return to this topic in more detail in Chapter 6.

\subsection{Error analysis}

Error measurements for dispersion curves measured from ambient noise correlations are easier to compute than for dispersion curves measured from surface waves produced by earthquakes, because the former can be measured repeatedly for different epochs whereas the latter can generally be measured only once along a particular path. However, fluctuations of noise source locations, amplitudes and spectra over time will also cause slight fluctuations in the cross-correlations. This can be regarded as a systematic error or used 
to advantage in studying time-dependent processes [e.g. Brenguier et al., 2008], but because it is difficult to assess the change in correlations due to a change in the noise source distribution, we treat this error statistically [for a discussion see Yao and Van Der Hilst, 2009]. Other sources of systematic errors include incorrect instrument response functions and instrument timing errors, which we address as carefully as possible. Random errors are mainly due to unavoidable imprecisions in the recording system and the signal processing which cannot be easily separated from errors due to fluctuations in the noise field, and we therefore regard both as causes for seasonal variations of dispersion curve measurements.

\subsubsection{Instrument responses and timing errors}

Applying ambient noise tomography to existing datasets from seismometer arrays that were not designed specifically for array analysis often poses major challenges for signal processing. Some of the main problems are the handling of different instrument types, detection of polarity reversals due to reversed wiring and instrumental timing errors, and treatment of heterogeneous azimuthal coverage and noise source distribution. During the four temporary deployments analyzed here, three different types of seismometers and three different types of dataloggers were employed. As our cross-correlation process only preserves phase information and disregards amplitude information we do not correct for different datalogger types. Some of the seismometer response functions of the CNIPSE stations had reversed polarities, which we discovered by comparing the P-wave polarity from a teleseismic earthquake filtered between 0.002 and $0.2 \mathrm{~Hz}$ (Figure $5.5 \mathrm{~A}$ and B). This was straightforwardly corrected by changing the sign of one of the zeros in the seismometer response function (Figure 5.5 C and D).

Once the instrument responses are known, we check for timing errors employing the method suggested by Sens-Schoenfelder [2008]. In this approach, the correlation between daily cross-correlations and the overall stack is calculated. If the maximum of the correlation is not zero and the correlation coefficient exceeds a certain threshold, then the daily correlation is shifted to align it with the overall stack. This can be repeated, improving the stacked correlation with each iteration. In practice, it turns out that only one iteration produces significant improvement. Figure 5.6 shows the result for one such iteration for the station pair TAUC (Tauhara) and LOPO (Opouahi Station). Although there are a few days with discernible timing errors (specifically days 1-10), the cross-correlation trace for the corrected daily correlations and the corresponding dispersion curve differs only 

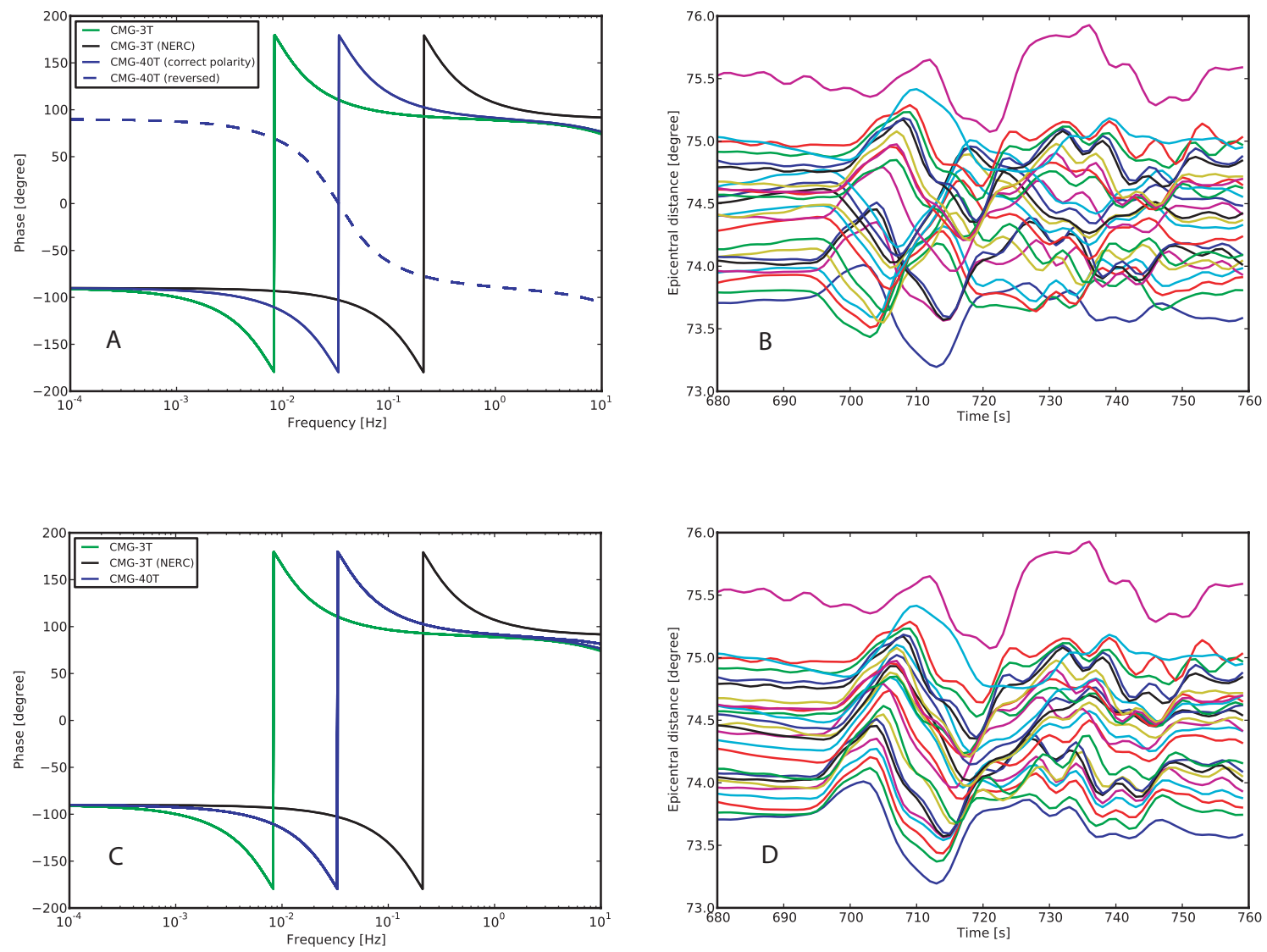

Figure 5.5 (A) Original phase response functions for the three different instrument types used during the CNIPSE experiment. Note that there are two different response functions for CMG-40T-type seismometers (blue lines) which have opposite polarity. (B) P-wave for 26 vertical recordings with the instrument response from the left panel removed and filtered between 0.002 and $0.2 \mathrm{~Hz}$. (C) Phase response functions for the three different instrument types used during the CNIPSE experiment and corrected for polarity reversals. There is now only one type of response functions for CMG-40T-type instruments (blue line). (D) P-waves for 26 vertical recordings with corrected instrument response removed and filtered between 0.002 and 0.2 $\mathrm{Hz}$. 
marginally from the uncorrected cross-correlation. An explanation is given by the fourthroot dependence of the signal-to-noise ratio versus the number of stacked days (Figure 5.7). Once the number of stacks exceeds a certain threshold, adding more daily correlations only improves the signal-to-noise ratio (SNR) slightly. Gerstoft et al. [2006b] observed a square-root dependence of the signal-to-noise ratio and the number of stacks for cross-correlations measured between stations in southern California. The analytic function, that describes the SNR as a function of the number of stacks, depends on the definition of SNR that is employed. In this study we define $S N R=10 * \log 10\left(S_{\text {max }} / S_{\text {noise }}\right)$, where $S_{\max }$ is the maximum amplitude of the cross-correlation and $S_{\text {noise }}$ the average amplitude of the trailing signal outside of an a priori surface wave arrival window which has a fourth-root function as the best fitting analytic curve. Defining the SNR as $S_{\text {max }} / S_{\text {noise }}$ results in a square-root dependence.

A limitation of the method of Sens-Schoenfelder [2008] is that it does not detect timing errors if all the daily correlations and consequently the stacked correlation itself are shifted by a constant amount. Such a constant shift could be caused by an erroneous phase response function, or a constant timing error at one of the stations, and would become apparent, when the causal and anticausal part of the stacked correlation function is not symmetrical in time.

\subsubsection{Dispersion curve uncertainties}

The conventional way of assessing the reliability of dispersion curves measured from ambient noise correlations is to measure dispersion curves from different substacks of the daily cross-correlations. As the noise sources vary over time, this should give an estimate of how reliably the dispersion information can be recovered given different source distributions. This approach works well for cross-correlation functions computed between the permanent GeoNet stations. However, the shorter the deployment times of an array, the more difficult it is to construct substacks of daily correlations that yield sufficient SNR values. Bensen et al. [2007] proposed using proxy-curves in this case, which relate the SNR to the standard deviation of the dispersion curves measured from the seasonal variability of noise correlations. The advantage of this approach is that a few long-duration noise recordings are sufficient to establish such a proxy curve, which can then be applied to station pairs with shorter recording times.

For the cross-correlations between temporary stations, we create substacks of 30 daily cross-correlations with an overlap of 20 days. Measuring six or more dispersion curves from these substacks is only possible for $\sim 80 \%$ of all possible station pairs. Therefore 

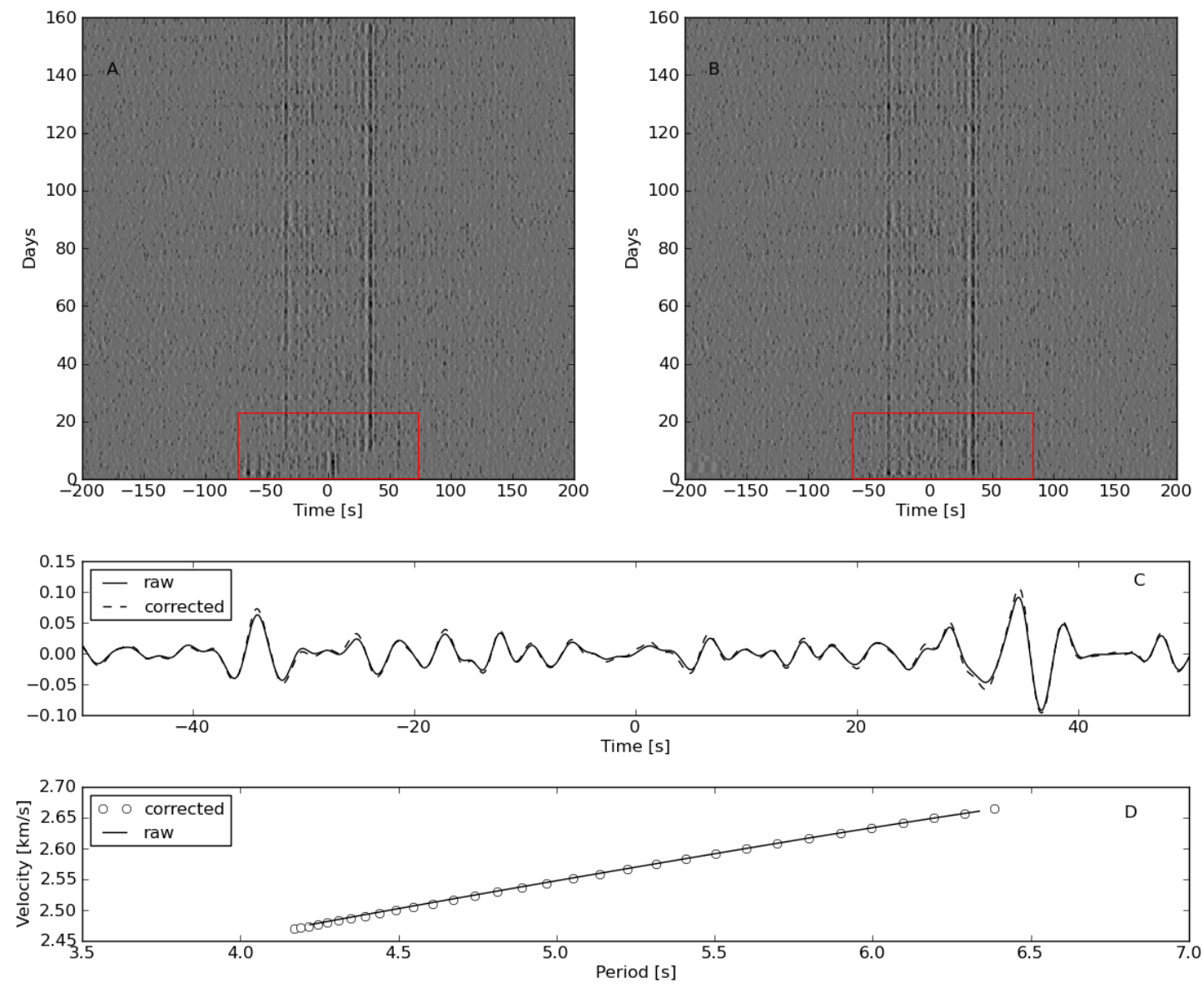

Figure 5.6 Example of timing correction using the method described by Sens-Schoenfelder [2008] for the station pair TAUC (Tauhara) and LOPO (Opouahi Station): (A) daily cross-correlations filtered between 2 and $10 \mathrm{~s}$ before correction; (B) daily cross correlations after correction; note the difference for the first 10 days highlighted by the red box; (C) stacked cross-correlations for uncorrected (solid line) and corrected (dashed line) daily correlations; (D) dispersion curves measured from the original cross-correlation (solid line) and the corrected cross-correlation (circles). 


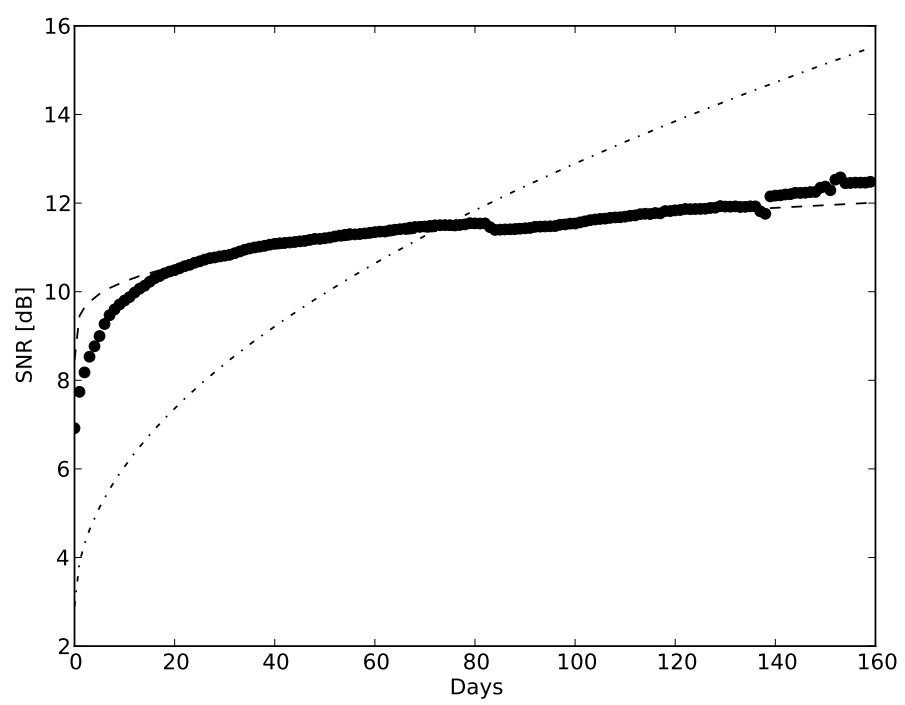

Figure 5.7 The points describe the mean SNR for a given number of daily stacks. The dashed black line shows a fourth root dependence. For comparison, the dash-dotted line shows the square root dependence observed by [Gerstoft et al., 2006b] for southern California.

we calculate average lower- and upper-bound dispersion curves for those station pairs for which we could measure six or more substack dispersion curves and relate this to an average SNR curve for the same station pairs. The average lower- and upper-bound dispersion curves between temporary stations are shown in Figure 5.8, colored according to the average SNR for each period. In Figure 5.9, the same signal-to-noise ratio points are plotted versus the mean of the lower- and upper-bound dispersion curves. SNR and mean error (i.e. half the distance between the lower- and upper-bound dispersion curves) are clearly anti-correlated and we fit this relation with exponential curves to generate proxy-curves. Love wave velocities with SNR below $5 \mathrm{~dB}$ and Rayleigh wave velocities with SNR below $7 \mathrm{~dB}$ will be disregarded in the following analysis (see chapter 5.4) and we therefore don't try to fit SNR smaller than 4 for Love waves and smaller than 2.5 and 5 for Rayleigh wave phase and group velocities respectively.

Another measure for the reliability of a dispersion curve is the time-symmetry of the causal and acausal part of a correlation function. Figure 5.10 shows the root-mean-square (RMS) differences between dispersion curves measured from the causal and acausal parts of the correlation functions between temporary stations and averaged over 542 crosscorrelation functions. We can see a clear dependence of RMS difference on period. The differences are lowest within the period band of the primary and secondary microseisms and increase quickly outside of this period range. Based on this and the consistency of our surface wave maps (Section 5.4), we restrict Rayleigh wave dispersion measurements to 

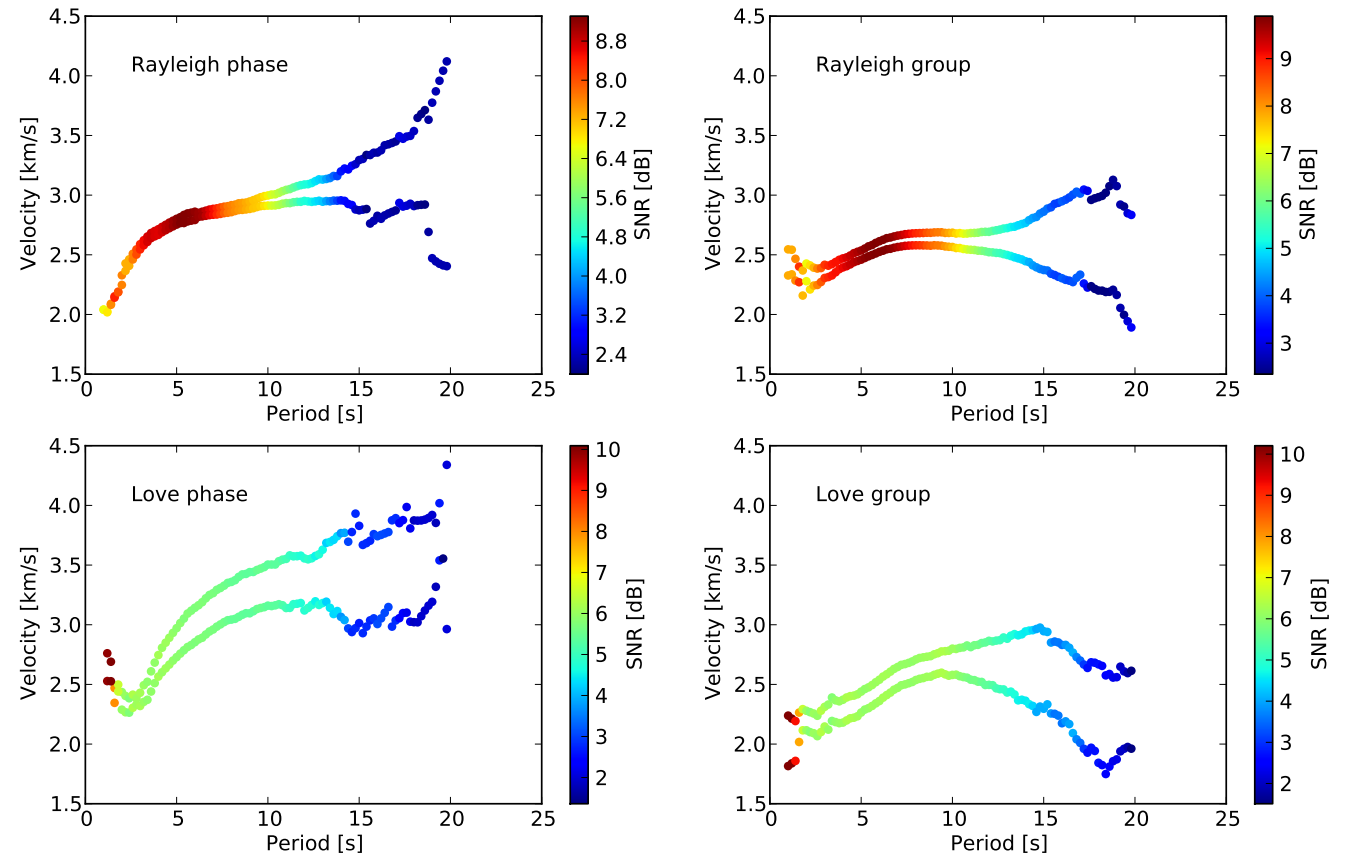

Figure 5.8 Average dispersion curves measured between stations of the temporary deployments for Rayleigh wave and Love wave phase and group velocities. The average SNR at each measurement point is shown in color. 

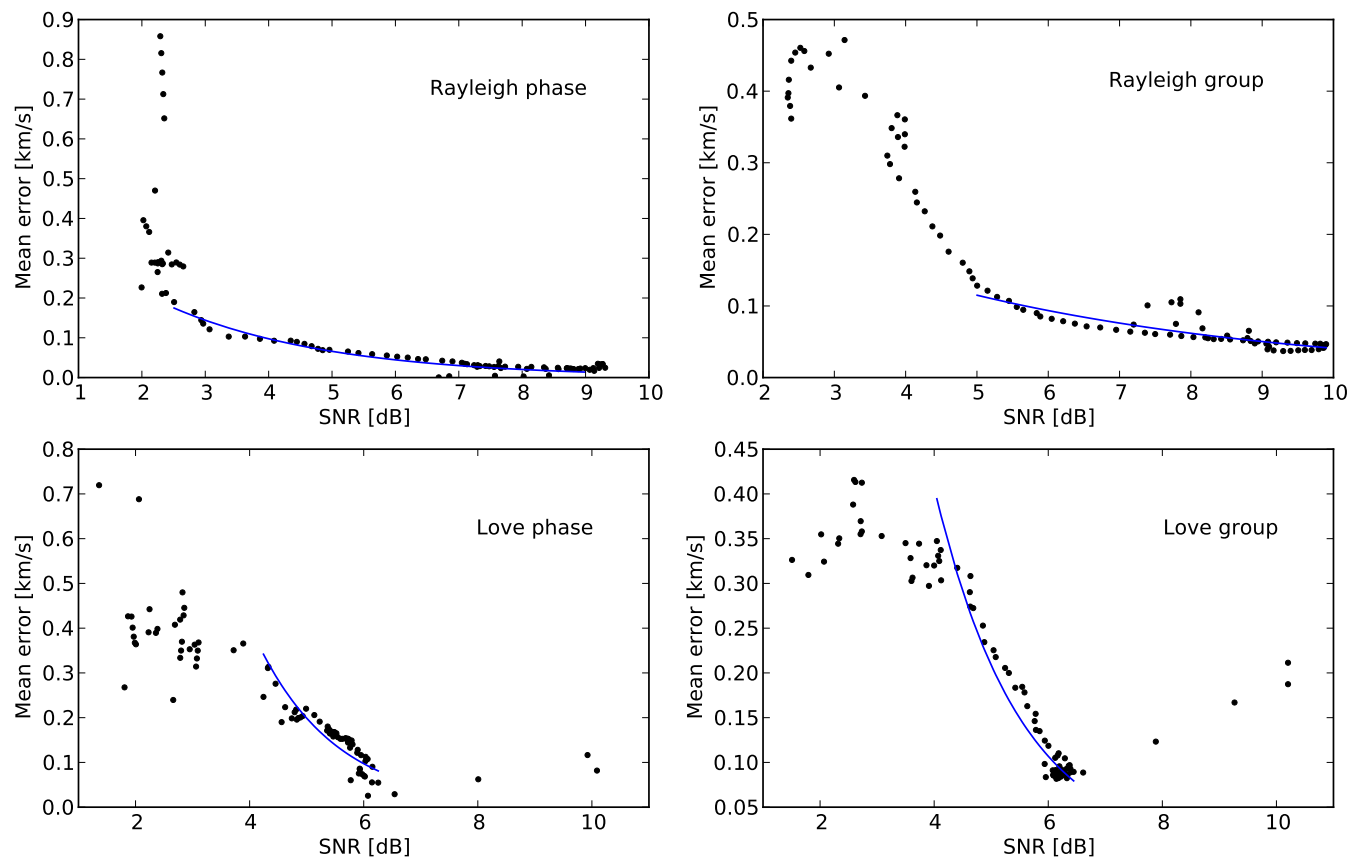

Figure 5.9 Mean error determined from the average dispersion curves in Figure 5.8 versus SNR (black dots) fitted with exponential curves (blue lines) for SNR greater than 4 for Love waves and greater than 2.5 and 5 for Rayleigh wave phase and group velocities respectively. 

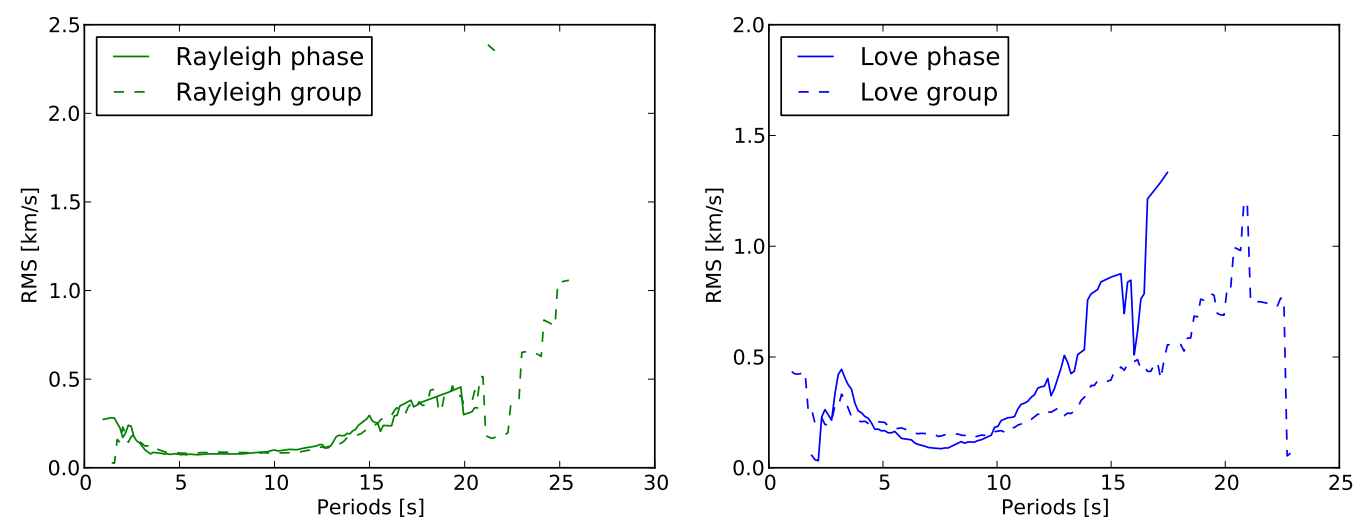

Figure 5.10 Left panel: RMS difference of Rayleigh wave phase and group velocities measured from the causal and acausal part of the cross-correlation functions. Right panel: The same as the left panel for Love wave phase and group velocity dispersion curves.

5-12 s period and Love wave dispersion measurements to 6-11 s period for correlations between temporary stations.

We use the error of our dispersion curves to estimate an error in the surface wave maps by inverting for maximum and minimum velocity values. In this way we obtain three surface wave maps at each period, which we then use to estimate the error of the dispersion curves constructed at each geographical grid point from the set of surface wave maps at successive periods. Figure 5.11 shows an example of dispersion curves with the corresponding errors for the point $-38.5^{\circ} / 176.0^{\circ}$ in the central TVZ.

\subsection{Tomography}

We calculate cross-correlation functions for all possible station pairs of our dataset and measure Rayleigh and Love wave phase and group velocities from the correlation functions. Figure 5.12 shows all possible interstation paths and the corresponding crosscorrelation functions as a function of interstation distance.

Following Stehly et al. [2009] we reject measurements from stations that are closer than two wavelengths to each other. At periods longer than $12 \mathrm{~s}$, this quality control criterion is not met by many paths between temporary stations, which reduces the path coverage for the subsequent analysis of lateral surface wave velocity variations. In order to extend the analysis to longer periods, we incorporate correlations between stations of the permanent seismic network, GeoNet. This does not fully overcome the lower path coverage at long periods relative to that at 5-12 s, as the GeoNet station distribution is relatively sparse, but 


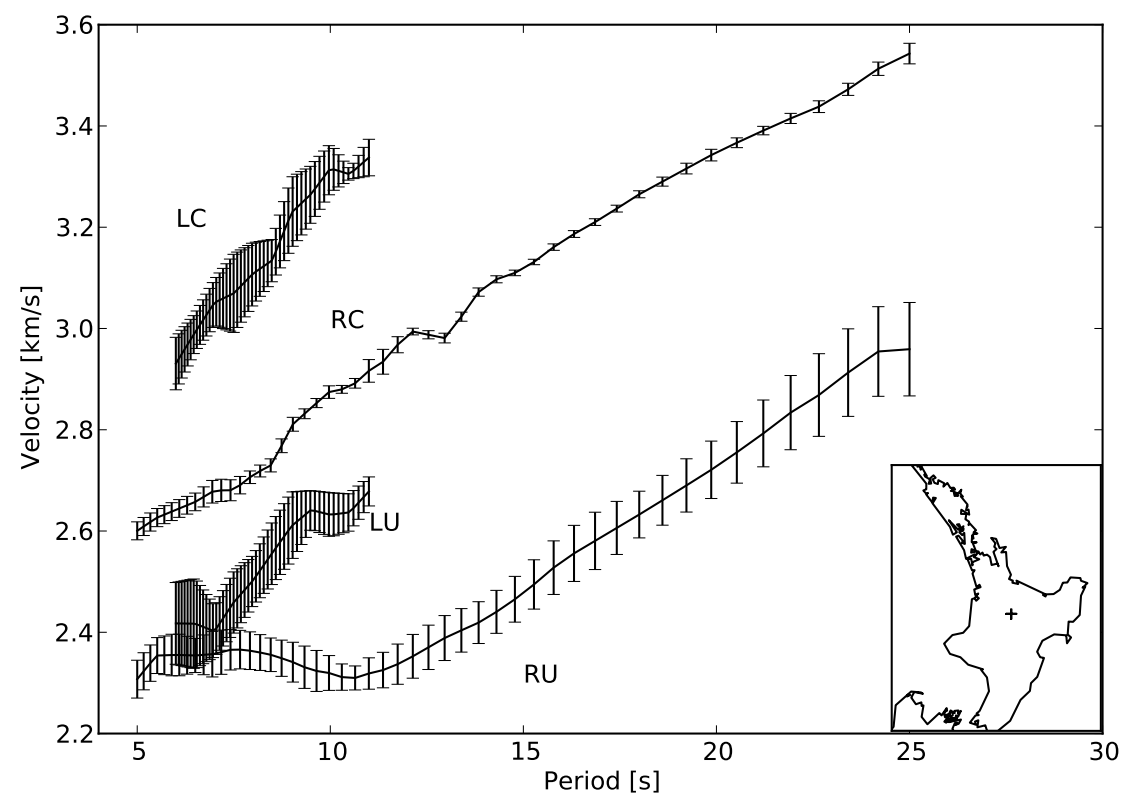

Figure 5.11 Dispersion curves at grid point $-38.5^{\circ} / 176.0^{\circ}$ (black cross in inset map) for Love wave phase (LC), Love wave group (LU), Rayleigh wave phase (RC) and Rayleigh wave group (RU) velocities and the corresponding error bars.
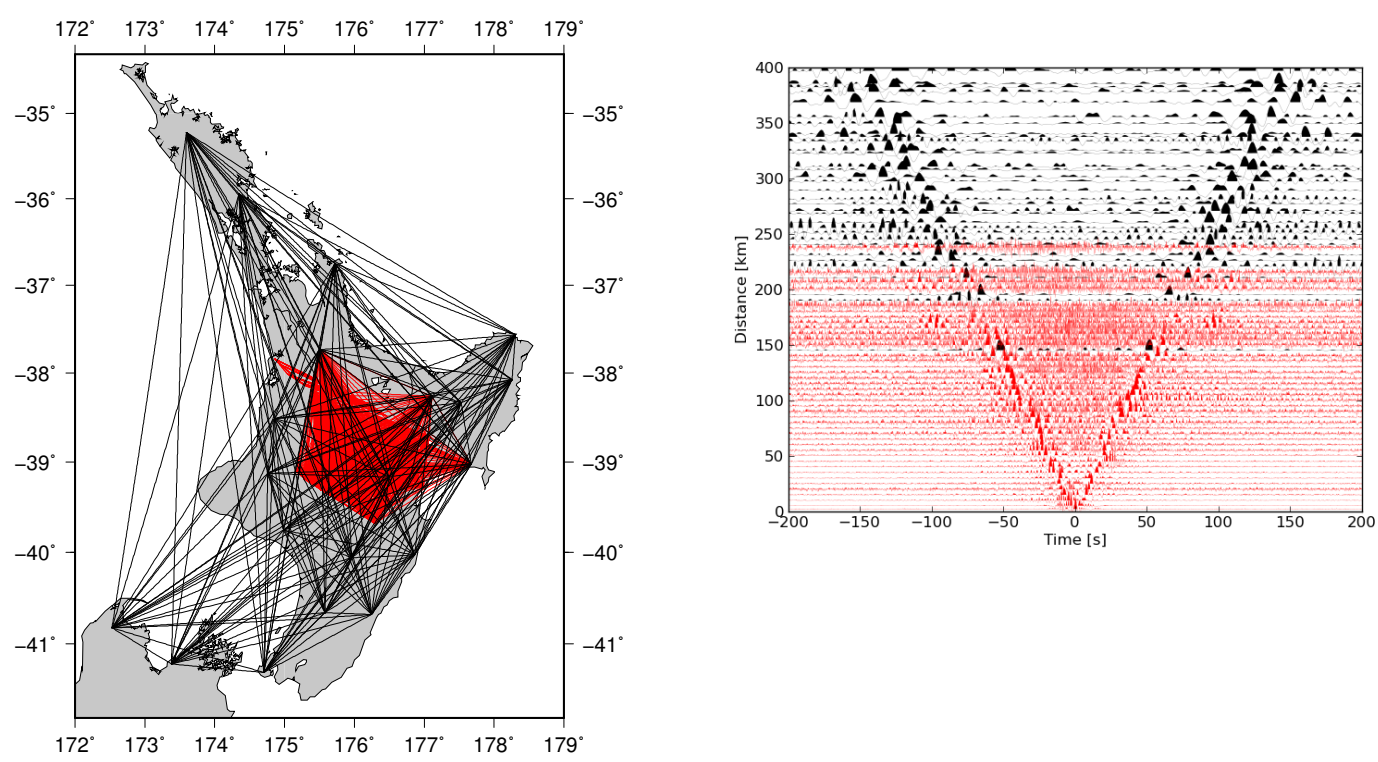

Figure 5.12 Interstation paths (left) and cross-correlation functions (right) for station pairs of the temporary deployments (red) and the permanent GeoNet stations (black). 
it does enable us to extend our analysis to $25 \mathrm{~s}$ period for Rayleigh waves. For Love waves, good measurements between permanent stations are too few to infer lateral variations and we therefore restrict the Love wave analysis below to dispersion curves measured between instruments of the temporary deployments.

Besides a minimum interstation distance of two wavelengths (see Section 2.3.3), we also require velocities to lie between 1 and $5 \mathrm{~km} / \mathrm{s}$, measurements from vertical-vertical correlations to have a minimum SNR of $7 \mathrm{~dB}$, and those from radial-radial and transversetransverse correlations to have a minimum SNR of $5 \mathrm{~dB}$. We adopt these values based on the trade-off between using only high-quality measurements and retaining as many paths as possible. SNR generally decreases below $\sim 5 \mathrm{~s}$ period, which is the reason why we only obtain reliable dispersion measurements above $\sim 5 \mathrm{~s}$ period. Figure 5.13 shows that in the central North Island for most periods the average SNR for transverse-transverse correlations is about $3 \mathrm{~dB}$ below the SNR for vertical-vertical correlations. This causes the subsequent measurement of Love wave dispersion curves to be more problematic than the measurement of Rayleigh wave dispersion curves at identical periods. Friedrich et al. [1998] proposed different excitation mechanisms for Rayleigh and Love waves from microseisms, which most likely cause the discrepancy in SNR between the transverse and vertical correlation functions. Both Rayleigh and Love waves are excited by direct coupling of ocean waves to the ocean bottom in the primary microseism band. However due to the retrograde elliptical particle motion in the ocean waves, Rayleigh waves are expected to gain more energy than Love waves. In the secondary microseism band Love waves are mainly excited by scattering of Rayleigh waves and therefore carry less energy than Rayleigh waves (see also Section 4.3). FTAN (see Chapter 2.3.2) introduces a further selection criterion by rejecting dispersion curves that are not smooth.

After applying these quality criteria and measuring surface wave dispersion curves using FTAN, we obtain a maximum of 526 phase velocity measurements at $6 \mathrm{~s}$ period and a minimum of 71 velocity measurements at 24 s period from the 1653 vertical-vertical correlation functions. From the 1563 transverse-transverse correlations we obtain a maximum of 216 phase velocity measurements at $6 \mathrm{~s}$ period and a minimum of 50 measurements at $11 \mathrm{~s}$ period. Measuring group velocities appears to be more stable than measuring phase velocities and results in $\sim 100$ more measurements at the same periods.

To estimate the lateral distribution of surface wave velocities at discrete periods we employ the damped least-squares inversion algorithm implemented by Barmin et al. [2001]. Damping ensures spatial smoothness of the inversion results and is realized by including two penalty functions, one ascertains that neighbouring model-nodes are correlated and 


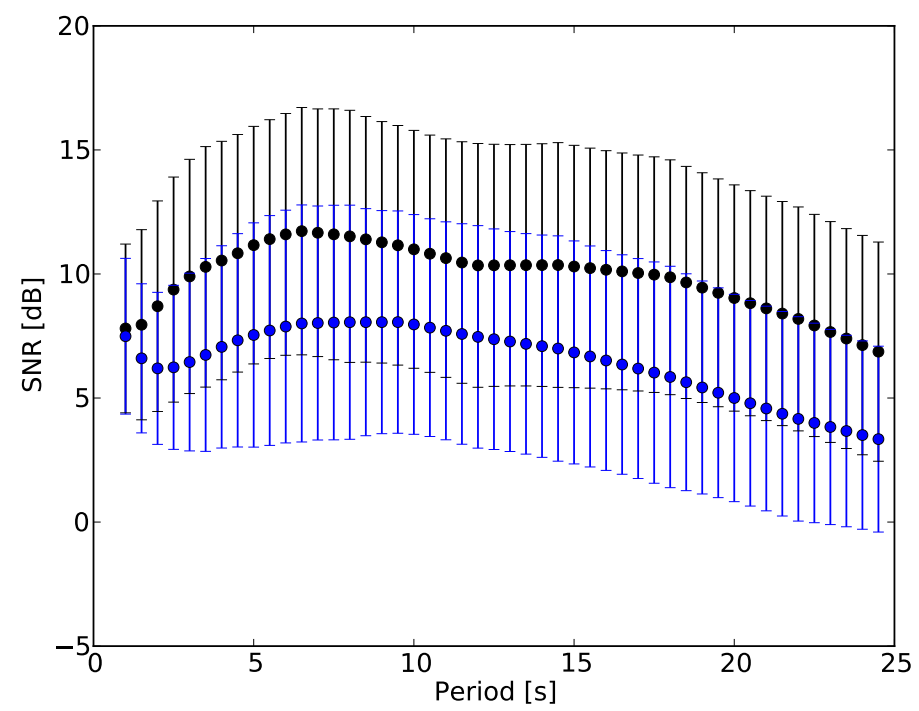

Figure 5.13 SNR versus period averaged for all vertical correlations (black dots) and transverse correlations (blue dots). Vertical uncertainties represent one standard deviation.

one downweights nodes with insufficient path coverage. The weighting of these functions and the length over which the models are required to be correlated are specified by the user. Figure 5.14 shows the variation of the mean and standard deviation of the misfit histogram as the weighting parameters representing spatial smoothing $(\alpha)$ and spatial correlation length $(\sigma)$ are varied systematically. We see that $\sigma$ exerts only a minor effect on the misfit statistics, which are mainly controlled by $\alpha$. Based on these tests we adopt $\alpha=200$ and $\sigma=100$, which represent the least possible damping while ensuring the misfit mean is close to zero and the standard deviation is small. The penalty function governing azimuthal coverage is controlled by the parameter $\beta$. This parameter mainly controls the boundary area of our velocity model, ensuring that it smoothly converges towards the average velocity in areas where azimuthal path coverage is low. Tests show that our results are not very sensitive to a change in $\beta$ and we therefore leave it at its standard value $\beta=1$ as suggested by Barmin et al. [2001].

We conduct resolution tests by exploring the characteristics of the resolution matrix following the method of Barmin et al. [2001], in which the resolution matrix is applied to a delta-like perturbation at each node of the inversion grid. This yields an estimate of the blurring inherent to the inversion procedure.

Figure 5.15 shows the inversion results at 6 s period of Rayleigh and Love wave phase and group velocities. Each map shows clearly three tectonic areas: slow velocities within the Taupo Volcanic Zone, which correspond to the thick volcaniclastic deposits; high 

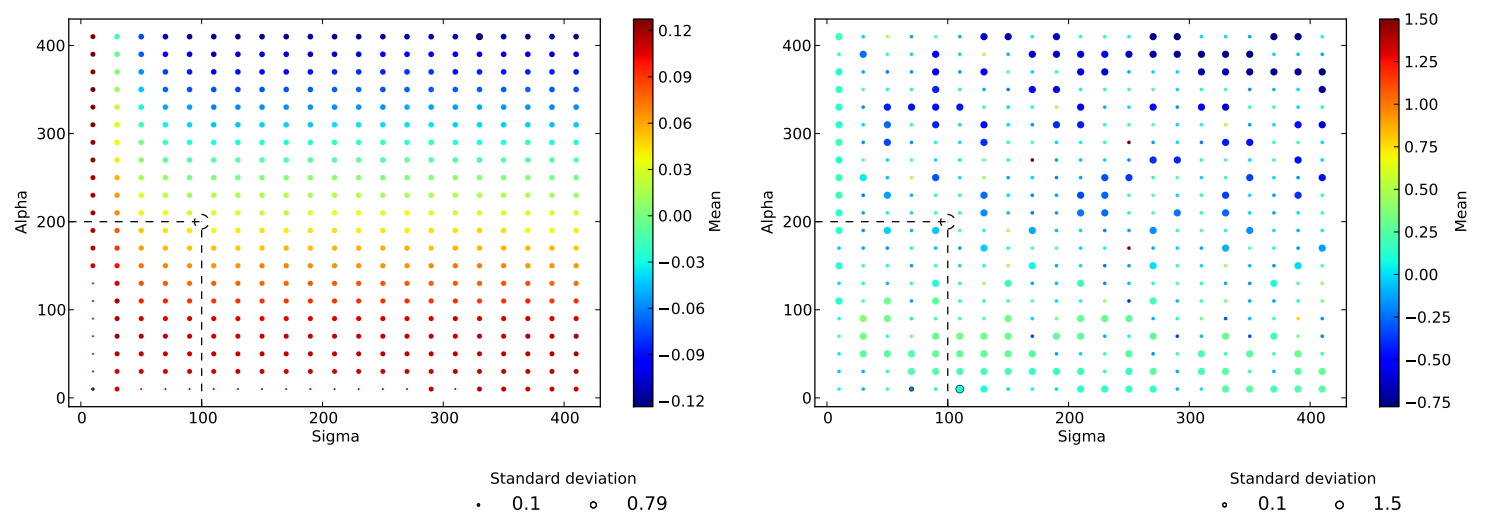

Figure 5.14 Systematic variation of spatial smoothing parameters $\alpha$ and $\sigma$ and the corresponding mean and standard deviation of the misfit histogram for the $6 \mathrm{~s}$ period Rayleigh wave phase velocity on the left and the $12 \mathrm{~s}$ period on the right. The dashed circle marks the set of parameters with the best compromise between highest resolution and lowest misfit variance.

velocities of the surrounding greywacke basement outcrops; and very slow velocities further east corresponding to the Wairoa Syncline, a $\sim 5 \mathrm{~km}$ deep sedimentary fore-arc basin [Field and Uruski, 1997]. An interesting difference between the Rayleigh and Love wave surface wave maps is the low-velocity region around Mt. Ruapehu visible in the former but not as clearly in the latter. Rowlands et al. [2005] reported the presence of low-velocity zones beneath and to the east of Mt. Ruapehu extending from the surface to depths of $\sim 10 \mathrm{~km}$, based on local earthquake tomography. One explanation for why this LVZ cannot be seen in the Love wave phase maps is horizontal smearing, which increases in regions of low path coverage, as discussed in more detail in Section 5.6. The region of highest resolution extends further for the Rayleigh wave maps, as they also include velocity measurements between permanent stations.

In addition to features observed at $6 \mathrm{~s}$, phase velocity maps for the $10 \mathrm{~s}$ period (Figure 5.16) show a low-velocity channel between the Wairoa Syncline and the TVZ for Rayleigh and Love wave phase velocities. This is most likely an artifact stemming from an insufficient number of crossing paths in this area. Also low velocities beneath Mt. Ruapehu are only weakly discernible on the Rayleigh wave group velocity map.

Comparing Rayleigh and Love surface wave maps serves as an important measure of quality control and it gives a first-degree estimate of the resolvable structures. The fact that all four maps in Figure 5.15 and 5.16 exhibit similar features provides evidence that our dispersion curve measurements and inversion results are consistent. To further test this, we compare phase velocity maps for Rayleigh waves measured from the vertical-vertical and radial-radial cross-correlations, which should yield identical velocities (Figure 5.17). As 
the radial-radial cross-correlation functions have lower SNR and therefore provide fewer high-quality dispersion curves than the vertical-vertical correlations, we reduce the number of paths for the inversion of the vertical component dispersion curves to match those from the radial component for their comparison. Although there are slight differences, the two maps in Figure 5.17 show the same lateral velocity variations overall.

\subsection{Pseudo-3D inversion}

\subsubsection{Methodology}

To construct pseudo-3D models from the measured dispersion curves, we follow the steps suggested by Shapiro et al. [2004]. At each grid point of our surface wave maps, we construct dispersion curves for group and phase velocities by interpolating between surface wave maps of successive periods. We then average dispersion curves over adjacent cells and jointly invert group and phase velocities for 1D S-velocity profiles at each cell using the Neighbourhood Algorithm [Sambridge, 1999a, b]. Finally, to construct a pseudo-3D volume from our set of $1 \mathrm{D}$ velocity profiles, we apply a linear interpolation.

This approach is an increasingly common way to invert surface wave data for 3D Svelocity structure. For example, Brenguier et al. [2007] used it to construct a 3D Svelocity model of the Piton de la Fournaise volcano on the island of La Réunion, and Moschetti et al. [2010] recently applied it to surface wave measurements from ambient noise correlations to estimate radial anisotropy in the crust and uppermost mantle of the western United States. A conceptually similar approach was described by Lévêque et al. [1998], who inverted surface wave seismograms for path-averaged 1D S-velocity profiles of the Indian Ocean and used these profiles to invert for lateral velocity variations in the mantle. Peter et al. [2008] used a similar approach but incorporated 2D finite-frequency kernels to improve the accuracy of the 2D surface wave maps.

Fully 3D inversions either involve solving the complete 3D wave equation [Tape et al., 2010] or using 2D [Marone et al., 2007; Feng and An, 2010] or 3D sensitivity kernels [Zhou et al., 2006]. The former requires an accurate 3D initial model and is therefore not applicable to our study area, where we try to establish such a model for the crust and uppermost mantle. The latter two only require spherically symmetric starting models but are computationally more involved and we have therefore chosen not to employ them for this work. However, in future work in the central North Island it would be interesting to investigate the effects of including finite-frequency kernels compared to the ray-theoretical 

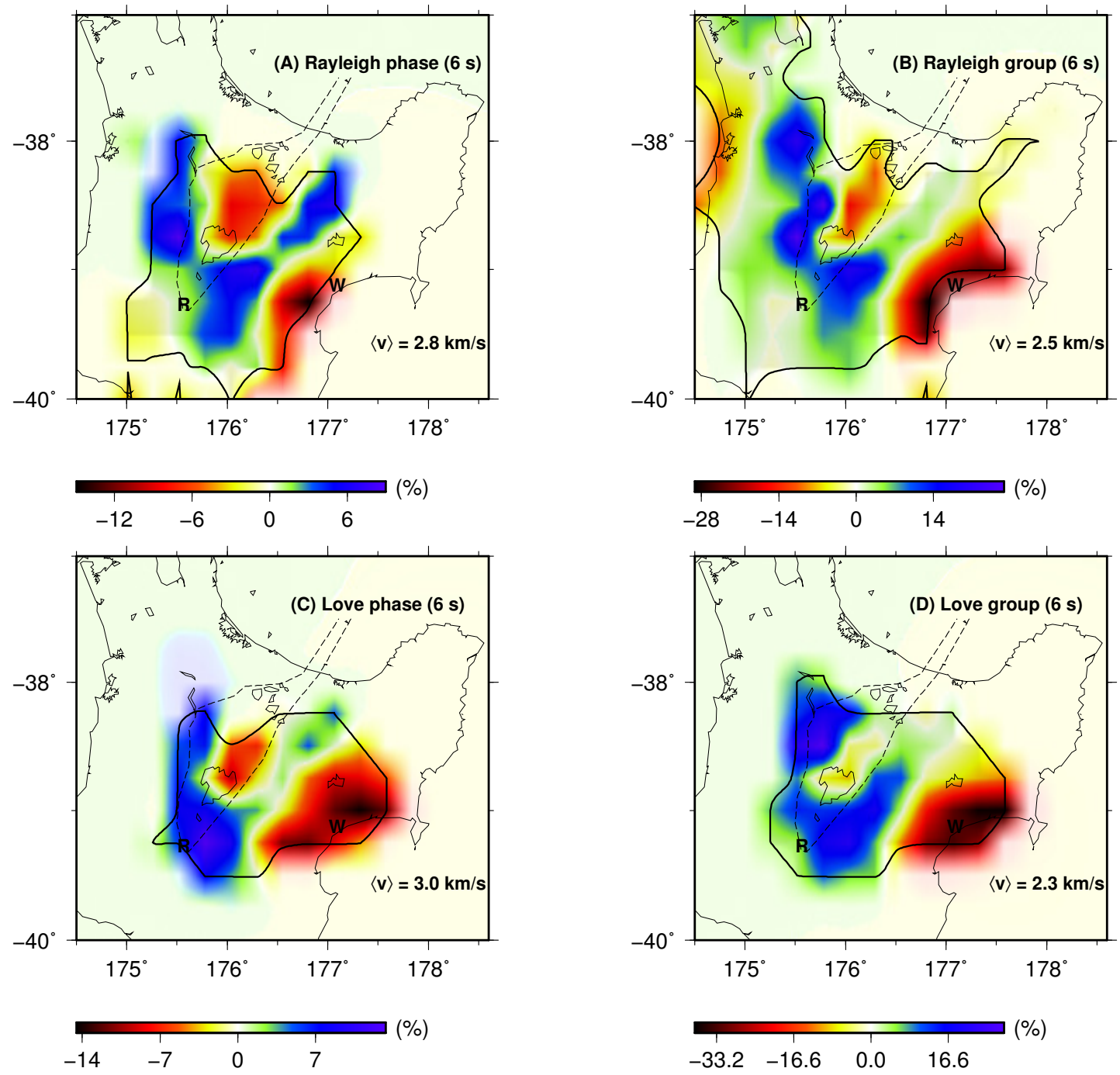

Figure 5.15 Surface wave velocity maps for Rayleigh wave phase (A) and group (B) velocity dispersion curves and for Love wave phase (C) and group (D) velocity dispersion curves at 6 s period. $<\mathbf{v}>$ specifies the mean velocity for each map and colors show the deviation of the surface wave velocities from this mean. The solid black lines mark the contour within which spatial resolution is below $100 \mathrm{~km}$ and the dashed black lines mark the outline of the Taupo Volcanic Zone (TVZ). Tectonic features: R=Mt. Ruapehu, W=Wairoa Syncline. 

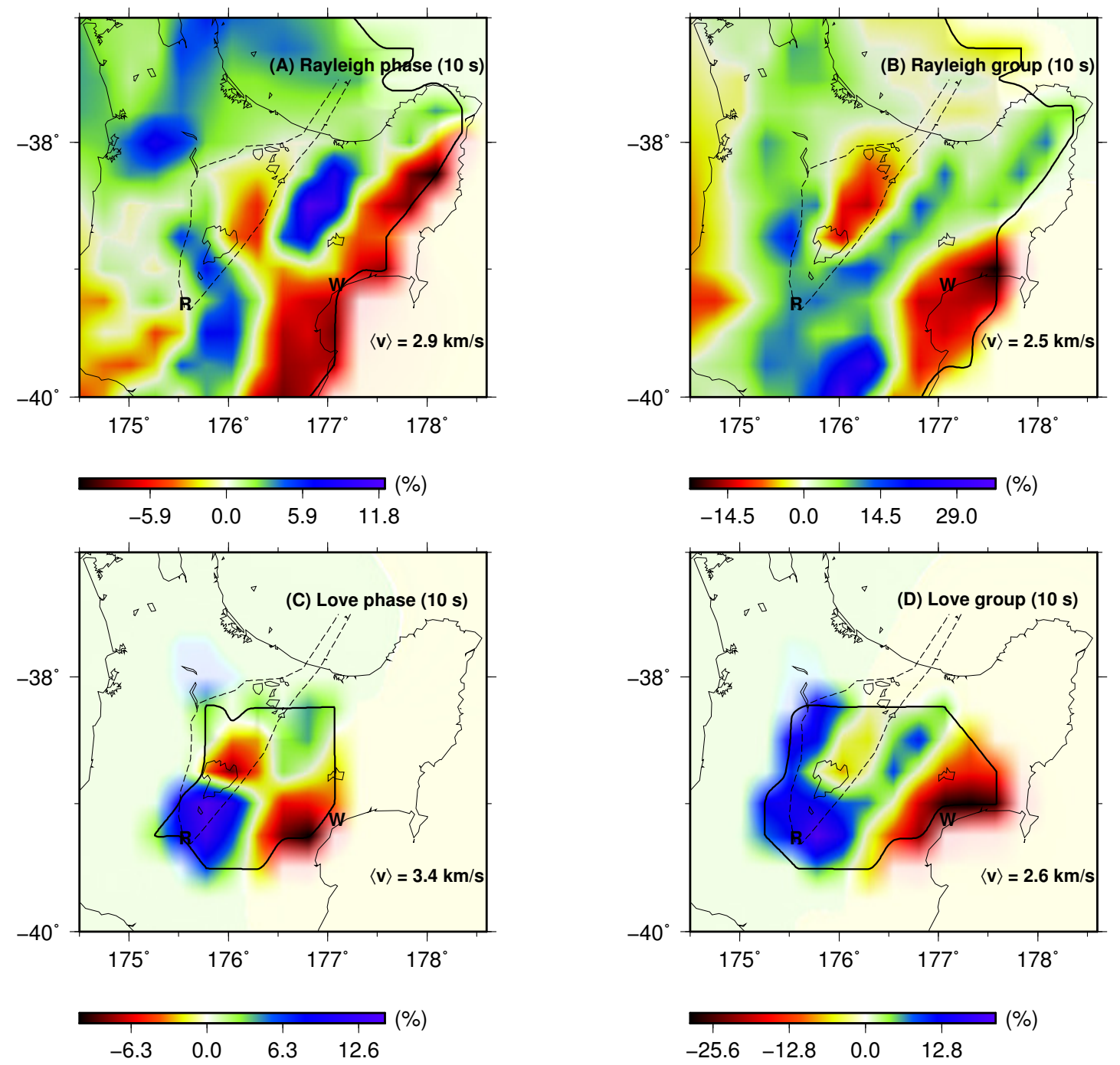

Figure 5.16 Same as Figure 5.15 for the 10 s period. 

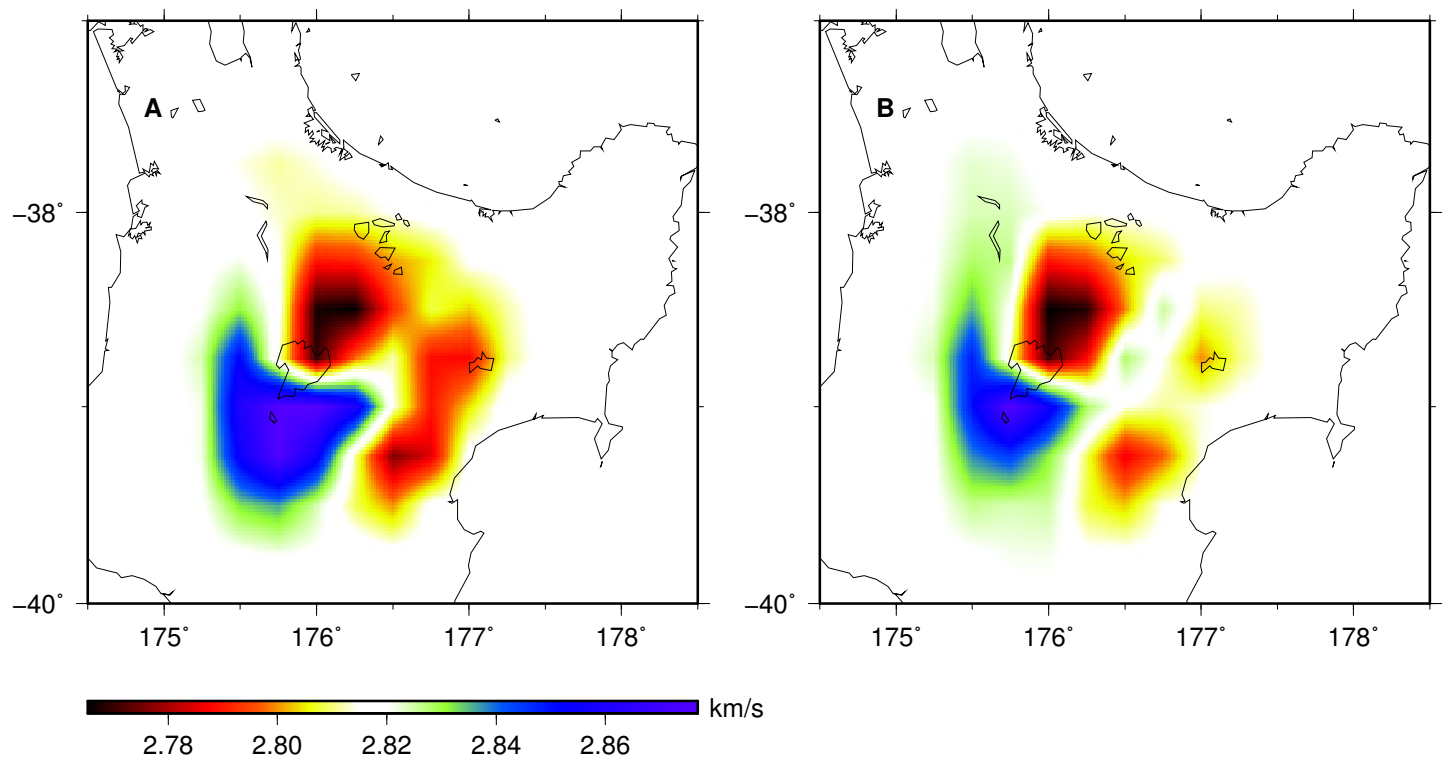

Figure 5.17 Comparison of inversion results for the $6 \mathrm{~s}$ period for Rayleigh waves measured from the vertical-vertical (A) and the radial-radial correlations (B).
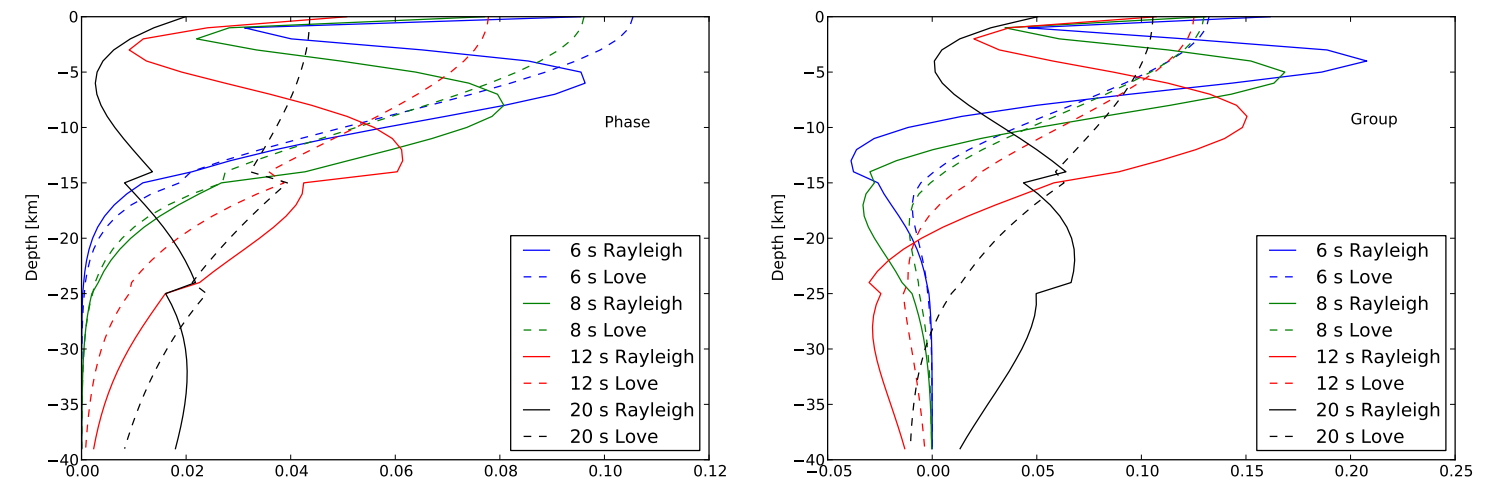

Figure 5.18 Sensitivity kernels calculated from PREM [Dziewonski and Anderson, 1981] for Rayleigh waves (solid lines) and Love waves (dashed line) at the $6 \mathrm{~s}$ (blue), $8 \mathrm{~s}$ (green) and $12 \mathrm{~s}$ period (red) and $20 \mathrm{~s}$ period (black). The left panel shows sensitivity kernels for phase velocities and the right for group velocities. 
approach used here. Ritzwoller et al. [2002] showed that for continental regions and periods shorter than $50 \mathrm{~s}$, models derived from ray-theory and finite-frequency theory are very similar.

We compute a pseudo-3D model for a parallelepiped of $40 \mathrm{~km}$ thickness for Rayleigh waves and $20 \mathrm{~km}$ for Love waves whose base area is the rectangle shown in Figure 5.1. This involves inferring 1D S- and P-velocities in 5 layers above a half space at 77 geographical points (spaced at $0.25^{\circ}$ ) and then amalgamated them using a linear interpolation. To calculate the $1 \mathrm{D}$ shear velocity profiles we employ the software package Dinver [www.geopsy.org; Wathelet, 2008] which combines the forward modelling algorithm of Dunkin [1965] with an improved version of the original Neighbourhood Algorithm and a least-squares misfit function.

\subsubsection{Model parameterisation}

Figure 5.19 shows the density distribution of all S-velocity models considered for three 1D inversions (see red stars in Figure 5.20). The top panels show the measured Rayleigh group and Rayleigh phase dispersion curves and their corresponding error bars on top of 300 synthetic dispersion curves that were drawn randomly from the 1000 best-fitting models of the corresponding ensembles of 30100 tested models. The models in the left panel and the middle panel result from the inversion at points in the central TVZ and the Wairoa Syncline, respectively. The models on the right panel were calculated for a point to the east of the TVZ, where greywacke outcrops at the surface. The boundaries of the colored area in the shear velocity panel mark the extent of the allowed search range and thereby a slice through the part of the parameter space that is searched by the Neighbourhood Algorithm. This is controlled by the a priori parameterisation, which is crucial to the outcome of the inversion process. Search ranges that are too large result in unstable results, while those that are too narrow can cause poor misfits as they might exclude the best-fitting solution. It is therefore important to introduce as much prior knowledge as possible. We divided our region of interest into two domains, each corresponding to a different parameterisation. The first domain is the volcanic area outlined by volcanic deposits in Figure 5.20 and the Wairoa Syncline, the thick sedimentary basin on the East Coast. Here we allowed for very low velocities at the top and for occurrences of low-velocity zones (LVZs) anywhere in the crust. Low velocities of the uppermost crust have been observed in many active source studies [e.g. Stratford and Stern, 2006; Bannister and Melhuish, 1997] and the presence of melts within the crust beneath the 
volcanic area may cause LVZs [Bannister et al., 2004, 2007]. The second domain is characterized by areas in which greywacke outcrops, such as the areas shown in blue colors in Figure 5.20. Here we assume much higher velocities at the top than for the East Coast domain and we do not allow LVZs. We allow P-velocities and layer thicknesses to vary around the model suggested by Stern and Benson [2011] for the central TVZ, except that the mid- and lower-crustal layer thickness of the S-velocity model are allowed to vary more widely. Apart from these differences the two parameterisations are identical. We see in all three inversions that the best-fitting model as well as the average model both lie well within the allowed parameter ranges. Furthermore the dispersion curves are all well fitted within their error bars. Several inversion runs with the same parameterisation lead to very similar results (not shown here) which suggest that the parameter space was not chosen too widely.

The only guarantee that the Neighbourhood Algorithm converges towards the global minimum is to search the whole parameter space. Since this is not feasible we have endeavored to use a parameterisation that is wide enough to encompass most physically reasonable models and consider the degree of bias this introduces to be less than that of linearized inversion methods requiring explicit starting models.

Another way to assess the quality of our parameterisation is to compare the spatial distribution of the best misfit values for different parameterisations. In Figure 5.21, we compare our preferred parameterisation with one in which we use the same parameterisation for all points and increase the number of layers but do not allow layer thicknesses to vary. We see that, in most areas, the misfit for the first parameterisation is equal to or better than for the second parameterisation. One has to bear in mind though, that a poor misfit does not necessarily result from an inappropriate parameterisation. For example, if the measured dispersion curves have kinks, attempting to fit those with a smooth dispersion curve will always result in large misfits even if the resulting S-velocity profile is physically reasonable and well resolved.

\subsubsection{Evaluation}

From the resulting pseudo-3D model we look at two 2D sections in more detail. One section is along the main branch of the CNIPSE/WCNIPSE deployment trending NW-SE and one is perpendicular to that following the trend of the TVZ between the Okataina volcanoes in the north and Mt. Ruapehu in the south in a NNE-SSW direction (Figure 5.20). Figure 5.22 shows the outline of the pseudo-3D volume together with the two $2 \mathrm{D}$ sections in a three-dimensional perspective plot with the direction of view southeast of 

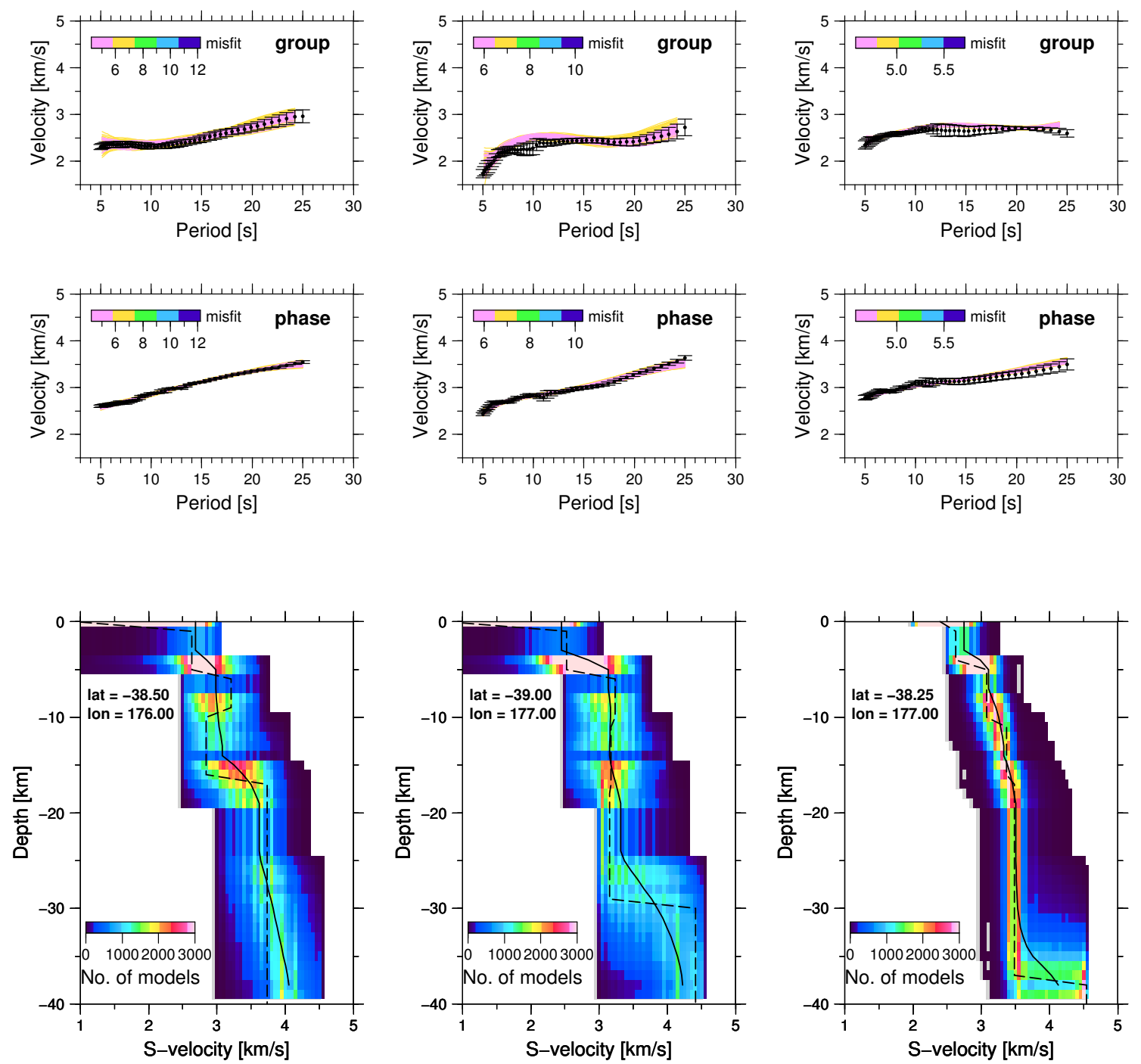

Figure 5.19 Inversion results for Rayleigh wave group and phase velocity dispersion curves measured at three different geographical points (red stars in Figure 5.20): central TVZ (left panels); the Wairoa Syncline (middle panels); and northeast of the TVZ (right panels). A different parameterisation was used for the model to the northeast of the TVZ than for the models in the central TVZ and the Wairoa Syncline. The colored areas illustrate the density distributions of all tested models. The thick black line represents the weighted average of the models plotted in color and the dashed line shows the best-fitting model. The top panels show the measured Rayleigh group and Rayleigh phase dispersion curves and their corresponding error bars (black dots with bars) on top of 300 synthetic dispersion curves that were drawn randomly from the 1000 best-fitting models of the corresponding ensemble. 


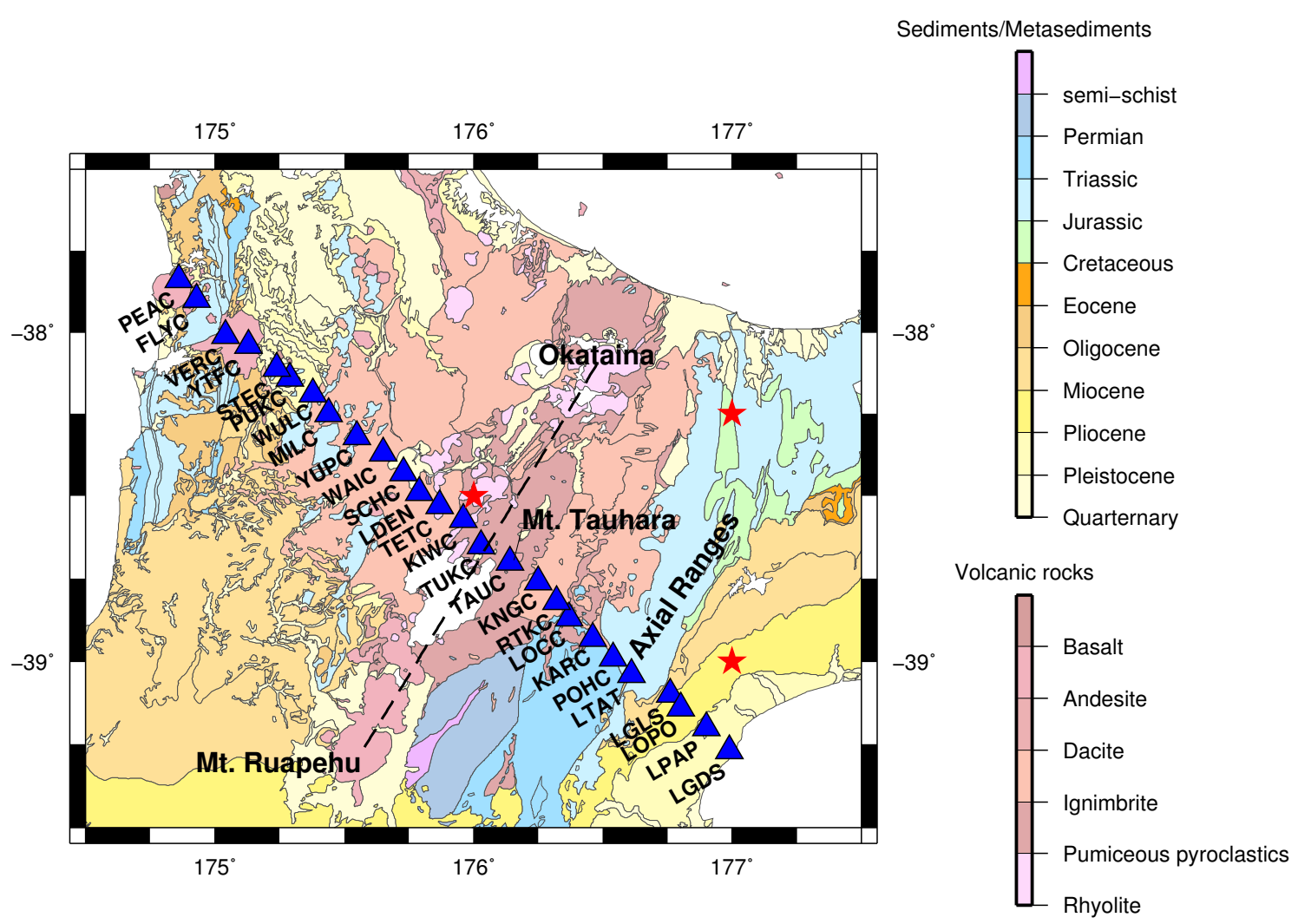

Figure 5.20 Geological map for the central North Island with the stations of the CNIPSE and WCNIPSE deployment (Geological data from the 1: 1000000 Geological Map of New Zealand (Officers of the New Zealand Geological Survey 1972) with limited updates by P.J. Forsyth and N. Mortimer (2004)). Off-line stations were omitted for clarity. Red stars mark the locations of the 1D models shown in Figure 5.19.
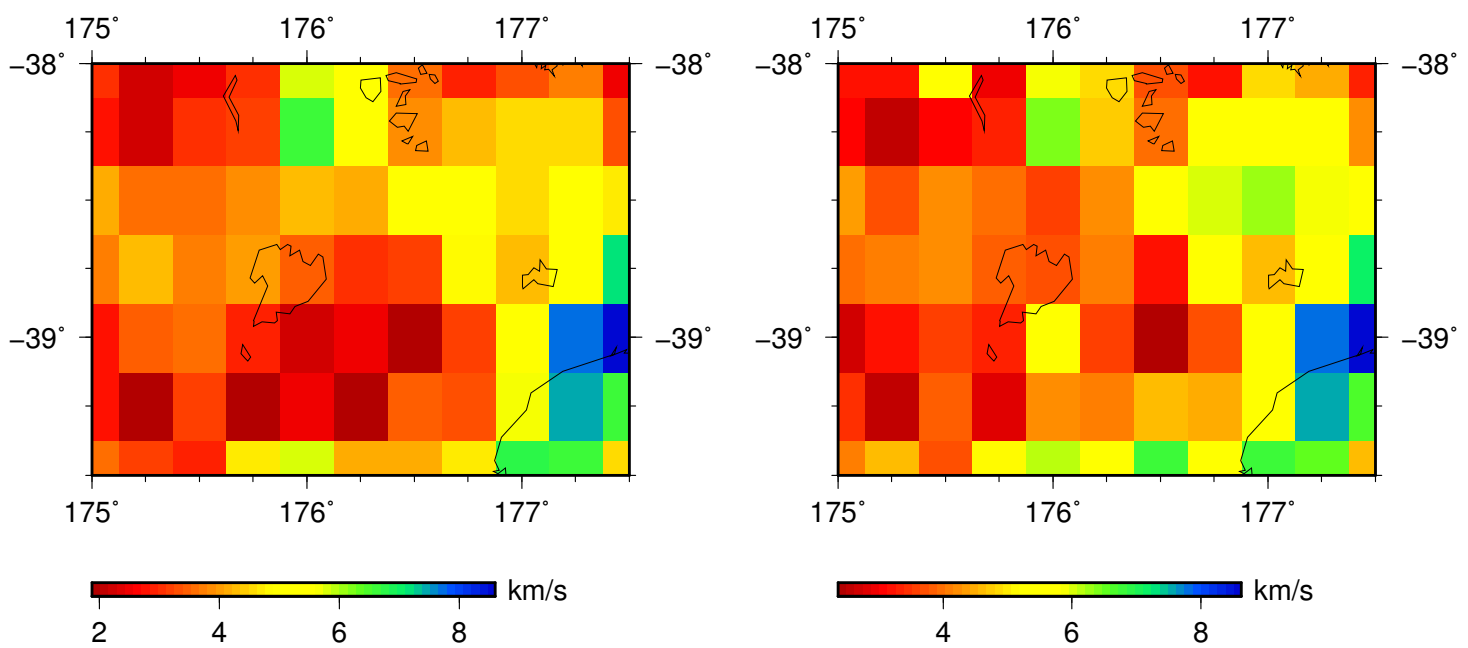

Figure 5.21 Misfit maps showing the best misfit between the synthetic and the measured dispersion curve for each nodal point of the pseudo-3D inversion. The left panel shows the misfits for our preferred parameterisation of 5 layers with variable thickness and the right panel for an alternative parameterisation of 11 layers with constant thickness. 


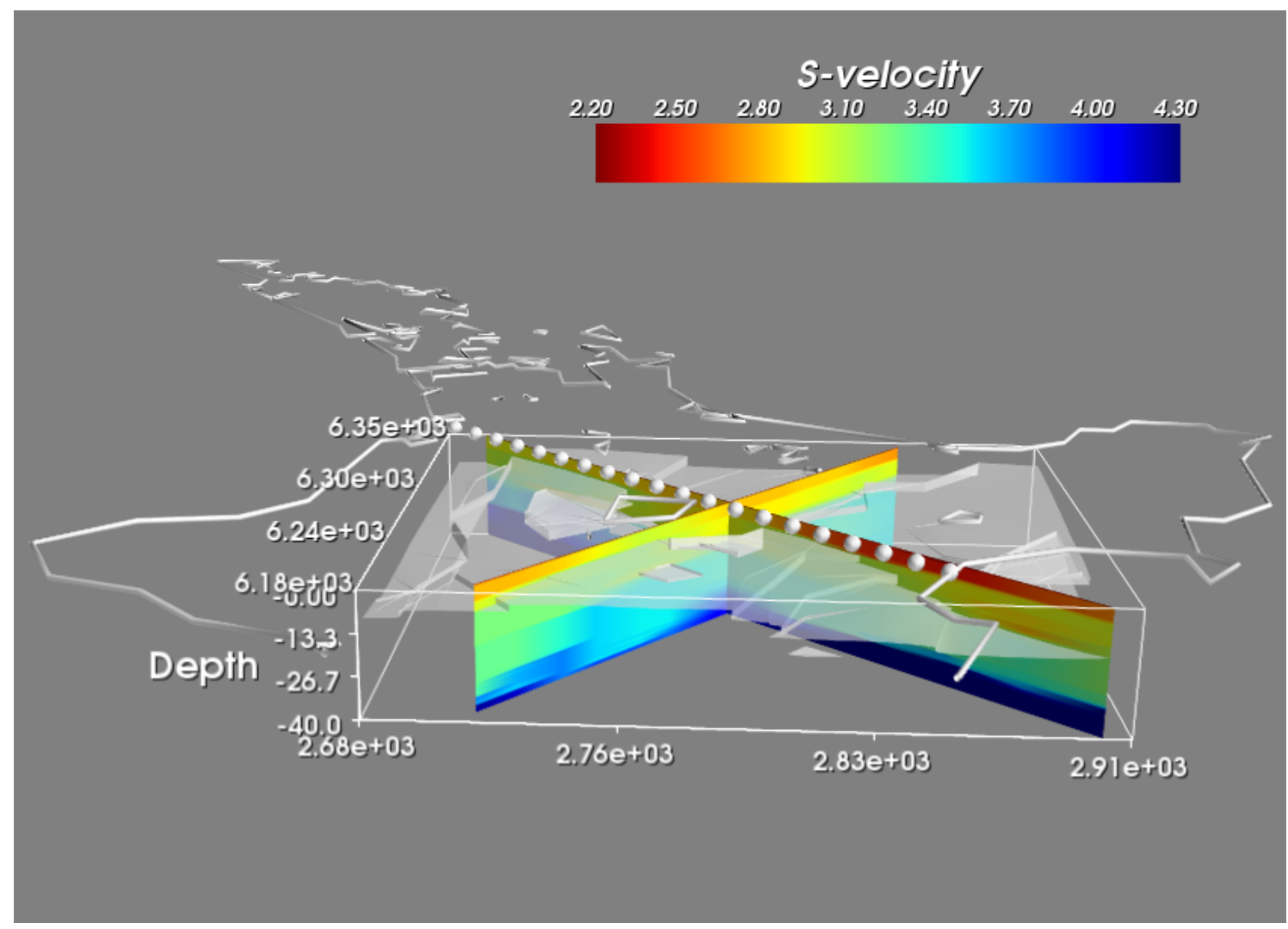

Figure 5.22 2D sections shown in Figure 5.23 and Figure 5.24 and iso-velocity surface at $3.1 \mathrm{~km} / \mathrm{s}$ from the pseudo-3D model for Rayleigh waves. The surface projection of the model is the square region illustrated in Figure 5.1, and the direction of view is approximately NNW from point $176^{\circ} /-40^{\circ}$. The line of spheres marks the locations of the CNIPSE and WCNIPSE stations (for clarity, off-line stations were omitted). Coordinates are in New Zealand Map Grid (NZMG).

the TVZ. It also shows the iso-velocity surface at $3.1 \mathrm{~km} / \mathrm{s}$ which we will discuss in more detail later.

The 2D section along the line of the CNIPSE/WCNIPSE deployment is shown on the top of Figure 5.23 above the Vs model calculated from the 3D Vp and $\mathrm{Vp} / \mathrm{Vs}$ models of Reyners et al. [2006]. Similarities between the two models are obvious below depths of $\sim 15 \mathrm{~km}$ where the fast lower crustal velocities at distances between 0 and $160 \mathrm{~km}$ along-strike decrease beyond $160 \mathrm{~km}$. The depth to upper-mantle velocities faster than $4.2 \mathrm{~km} / \mathrm{s}$ increases in the southeastern parts of both sections by about $5 \mathrm{~km}$ compared to that in the northwestern parts. Furthermore, we see in both sections the edge of the Wairoa Syncline, the sedimentary basin on the East Coast, between 170 and $220 \mathrm{~km}$ along-strike distance and the start of the sedimentary deposits to the west between 0 and $30 \mathrm{~km}$. The most striking differences between the two models are observed between 20 and $140 \mathrm{~km}$ along-strike distance at depths shallower than $\sim 15 \mathrm{~km}$. The S-velocity model from surface waves shows pronounced low velocities of $2.6-3.1 \mathrm{~km} / \mathrm{s}$ beneath the TVZ 


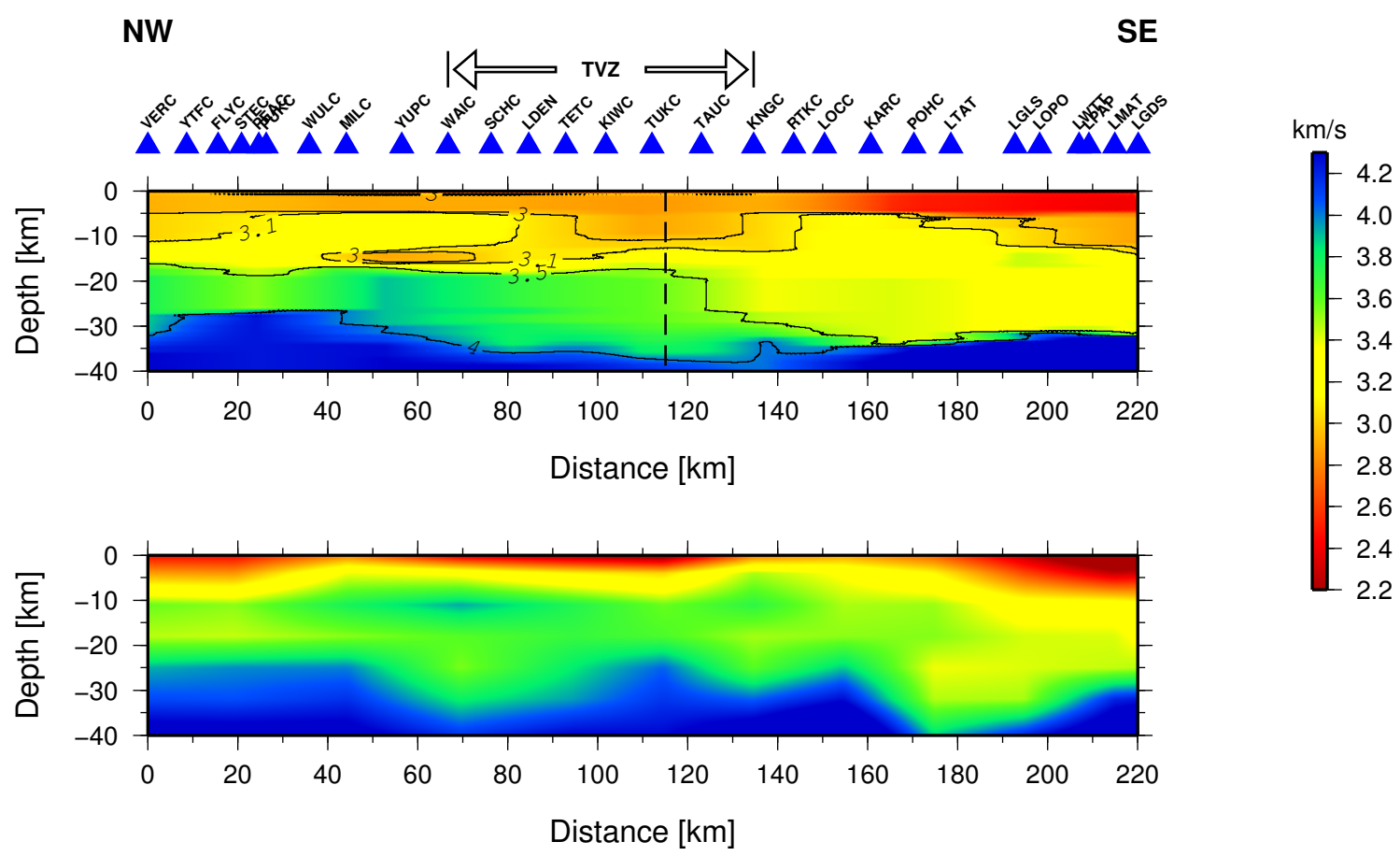

Figure 5.23 S-velocity vs. depth profile beneath the CNIPSE/WCNIPSE line (see Figure 5.20). The upper panel is a vertical section through the 3D S-velocity model calculated from Rayleigh wave maps between 5 and $25 \mathrm{~s}$ period. Contours are drawn at the boundaries of the $3 \mathrm{~km} / \mathrm{s}, 3.1 \mathrm{~km} / \mathrm{s}, 3.5 \mathrm{~km} / \mathrm{s}$ and $4 \mathrm{~km} / \mathrm{s}$ $\mathrm{S}$-velocity. The lower panel shows the S-velocity model for the same profile calculated from the $3 \mathrm{D} \mathrm{Vp}$ and Vp/Vs model of Reyners et al. [2006], who used a horizontal grid-spacing of $20 \mathrm{~km}$ and, for the depth range shown here, a vertical grid spacing of $4 \mathrm{~km}$ for the uppermost layer of nodes and then 7, 7, 7, 7 and $8 \mathrm{~km}$ for the following layers. For a detailed discussion on the resolution of their model see Reyners et al. [2006] and for uncertainties of the S-velocity model from surface waves see Figure 5.26. The black dashed line marks the intersection with the profile along the strike of the TVZ (Figure 5.24).

and a low-velocity zone extending to the northwest as far as the $\sim 60 \mathrm{~km}$ along-strike point. Velocities in the model of Reyners et al. [2006] are very low in the top 2-3 km but then increase rapidly from 3.2 to $3.8 \mathrm{~km} / \mathrm{s}$ with one lens-shaped area at $\sim 70 \mathrm{~km}$ alongstrike distance and $10 \mathrm{~km}$ depth as fast as $4.0 \mathrm{~km} / \mathrm{s}$. It has to be noted that the model by Reyners et al. [2006] is inferred from a combination of active and passive source Pand S-wave arrivals and therefore is best resolved at depths where many earthquakes occur and above the maximum penetration depth for waves from active source studies. As most earthquakes beneath the TVZ occur either above $\sim 10 \mathrm{~km}$ or in the subducted Pacific plate, significant smearing occurs between 10-30 km depth [Reyners et al., 2006]. Furthermore, as $\mathrm{Vp} / \mathrm{Vs}$ values will be close to the initial value of 1.75 in areas of poor $\mathrm{S}$-wave path coverage the $\mathrm{Vs}$ values inferred from the $\mathrm{Vp}$ and $\mathrm{Vp} / \mathrm{Vs}$ model are strongly influenced by the Vp model in these regions. The comparison between the two models is therefore somewhat indirect, particularly at shallower depths, but nevertheless gives us valuable insights on the overall reliability of our results and on differences between the two models. 
Figure 5.24 shows a 2D section along the strike of the TVZ from the Okataina volcanoes to Mt. Ruapehu volcano (see Figure 5.1 and the dashed line in Figure 5.20) and a comparison with the model of Reyners et al. [2006]. Mid- to lower-crustal velocities in both sections are again similar apart from a zone of lower velocities between 20 and $40 \mathrm{~km}$ alongstrike and at 20 to $30 \mathrm{~km}$ depths. The change to upper-mantle velocities appears to occur at greater depths in the surface wave model than in the body wave model. Larger differences can be observed again at depths shallower than $\sim 15 \mathrm{~km}$ with low velocities of less than $3.1 \mathrm{~km} / \mathrm{s}$ in the surface wave model extending down to $\sim 15 \mathrm{~km}$ below Mt. Tauhara and shallowing out to about $11 \mathrm{~km}$ towards Okataina volcano and $5 \mathrm{~km}$ towards Mt. Ruapehu volcano. The body wave inversion of Reyners et al. [2006] shows again low velocities of less than $2.6 \mathrm{~km} / \mathrm{s}$ at the top but then changes from $\sim 3.2 \mathrm{~km} / \mathrm{s}$ to $\sim 3.6 \mathrm{~km} / \mathrm{s}$ between 3 and $15 \mathrm{~km}$ depth. As in the surface wave inversion, a shallowing of the slow velocities towards Mt. Ruapehu beyond $\sim 120 \mathrm{~km}$ distance can be observed.

The parameterisation of the 1D surface wave inversion requires any velocity change in the uppermost crust to happen between 0.1 and $1 \mathrm{~km}$ or 4 and $6 \mathrm{~km}$ depth. This results in the almost constant thickness of the uppermost layer observed in Figure 5.23 and 5.24. The 1D inversion results show that this leads to a satisfactory misfit and therefore doesn't restrict the search range too much while keeping the searchable parameter space as small as possible.

The surface corresponding to the $3.1 \mathrm{~km} / \mathrm{s}$ contour, which marks the jump from low to more typical upper-crustal velocities, is shown in Figure 5.25 as contour lines above the 1:1,000,000 geological map for the central North Island (Geological data from the 1:1,000,000 Geological Map of New Zealand (Officers of the New Zealand Geological Survey 1972) with limited updates by P.J. Forsyth and N. Mortimer (2004)). It shows clearly the correlation between downwarping of the $3.1 \mathrm{~km} / \mathrm{s}$ contour and the sedimentary basin on the East Coast as well as the Maroa caldera north of Lake Taupo, one of the major rhyolitic centres that was active in the last 65 Kyr [Wilson et al., 1995].

Employing the Neighbourhood Algorithm gives us the opportunity to assess the uniqueness of our inversion by evaluating the range of different models that fit the observation within certain misfit bounds. To quantify the variability in velocity we calculate the histogram of the S-velocity at depth intervals of $0.5 \mathrm{~km}$ for the 1000 best fitting models of each 1D inversion. We then fit a Gaussian curve to every histogram and define one standard deviation to be the measure of variability. Figure 5.26 shows on the two top panels the standard deviation at each point of the NW-SE and NNE-SSW cross-sections. Both show the highest variability at depths of $\sim 15 \mathrm{~km}$ and $\sim 30 \mathrm{~km}$. The strong variability at these depths can be caused by the error increase of the dispersion curves at periods around 


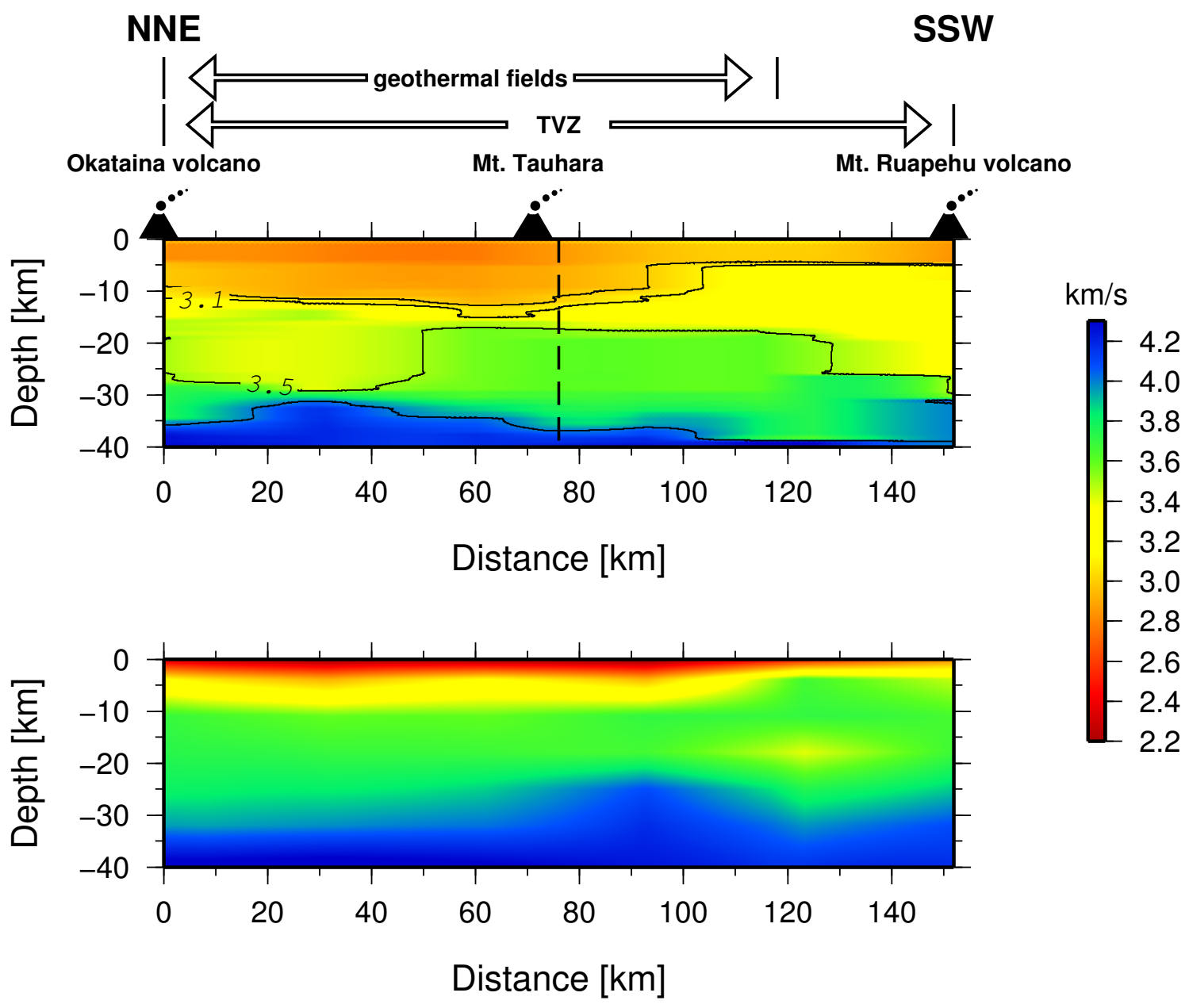

Figure 5.24 S-velocity vs. depth profile along-strike of the TVZ between Okataina volcano and Mt. Ruapehu volcano (see Figure 5.20). The upper panel is a vertical section through the 3D S-velocity model calculated from Rayleigh wave maps between 5 and 25 s period. The lower panel shows the S-velocity model for the same profile calculated from the 3D Vp and Vp/Vs model of Reyners et al. [2006], who used a horizontal grid-spacing of $20 \mathrm{~km}$ and, for the depth range shown here, a vertical grid spacing of $4 \mathrm{~km}$ for the uppermost layer of nodes and then 7, 7, 7, 7 and $8 \mathrm{~km}$ for the following layers. For a detailed discussion on the resolution of their model see Reyners et al. [2006] and for uncertainties of the S-velocity model from surface waves see Figure 5.26. The black dashed line marks the intersection with the profile beneath the WCNIPSE/CNIPSE line (Figure 5.23). 


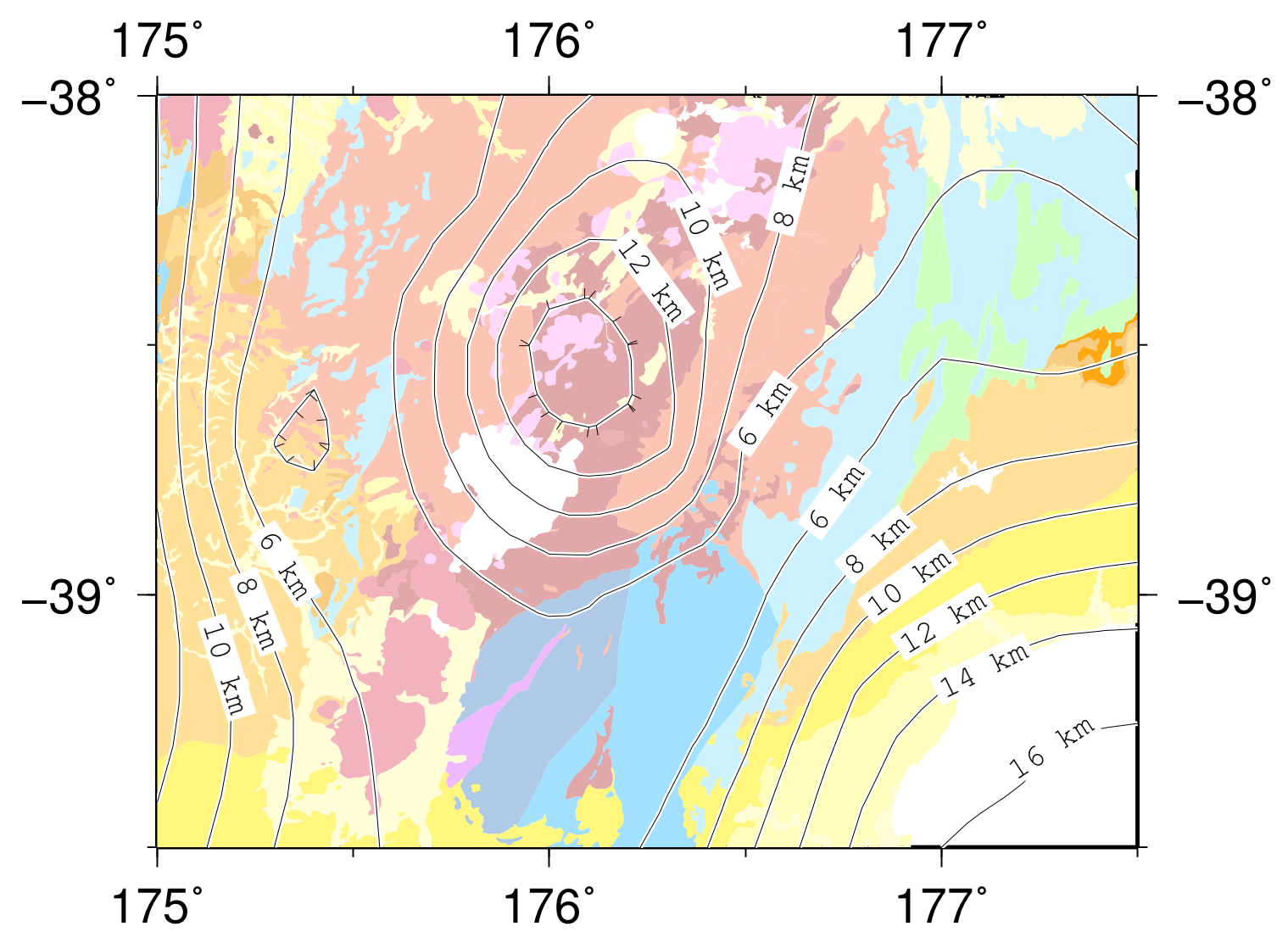

Figure 5.25 Isosurface of the $3.1 \mathrm{~km} / \mathrm{s} \mathrm{S}$-velocity calculated from the pseudo-3D S-velocity model. 
$10 \mathrm{~s}$ and above $20 \mathrm{~s}$ period (see Figure 5.19), which have high sensitivity at mid-crustal and lower-crust/upper-mantle depths respectively (see Figure 5.18). The depth uncertainty is evaluated by calculating the histogram of the depth of each of the 5 layers above the half space, defined in the parameterisation, for the 1000 best fitting models and then fitting again a Gaussian curve to every histogram. The resulting standard deviations are then interpolated at $0.5 \mathrm{~km}$ intervals to create a $1 \mathrm{D}$ profile of standard deviation at every geographical point in the inversion grid. The two bottom panels in Figure 5.26 show these 1D profiles linearly interpolated onto the NW-SE and NNE-SSW cross-sections. Depth variability above $\sim 15 \mathrm{~km}$ is less than $1.5 \mathrm{~km}$ and increases to up to $5.5 \mathrm{~km}$ beneath the TVZ and approximately $2 \mathrm{~km}$ beneath the regions to the east and west of the TVZ. The increase of variability with depth is what we expect from the decreased depth sensitivity of Rayleigh waves due to the cutoff period of $25 \mathrm{~s}$. High variability can also be an indicator for heterogeneous structures that are not well represented by a 1D S-velocity profile.

As discussed above, the measurement of Love waves is more problematic than Rayleigh waves and we were only able to measure reliable Love wave dispersion curves for periods of 6-11 s. This restricts our analysis of Love waves to the upper and middle crust, as their sensitivity diminishes severely at depths deeper than $\sim 20 \mathrm{~km}$ for periods of less than 12 $\mathrm{s}$ (see Figure 5.18). Furthermore it proved difficult to fit Love phase and group velocity dispersion curves with the same S-velocity model and we therefore inverted phase and group velocities separately. Figure 5.27 shows the S-velocity model inferred from Love wave group velocities along the NW-SE profile in comparison to the first $20 \mathrm{~km}$ of the $\mathrm{S}$-velocity model inferred from Rayleigh waves (Figure 5.23). Its most striking feature is a broad LVZ extending from approximately $40-150 \mathrm{~km}$ along-strike distance and situated between 10 and $20 \mathrm{~km}$ depth. This LVZ has the same length as the zone of low midto upper-crustal velocities in the Rayleigh wave model but is surrounded by structures with velocities of $\sim 3.2 \mathrm{~km} / \mathrm{s}$. The low velocities to the southeast of the profile, which correspond to the Wairoa Syncline, extend to slightly greater depths compared to the Rayleigh wave model but show similar shape. The slow velocities visible in the Rayleigh wave model down to $\sim 10 \mathrm{~km}$ depth at the northwestern end of the profile are not observed in the Love wave model. This is most likely due to the poor path coverage in this area for Love waves (see Figure 5.15 and 5.16).

The comparison of the S-velocity models inferred from Love and Rayleigh waves for the NNE-SSW profile is shown in Figure 5.28. As in the NW-SE profile, the extent of the slow-velocities along the strike of the profile is similar for the Rayleigh and Love wave model but the slow velocities in the Love wave model are confined to the depth range of 10-20 km and are most pronounced beneath Taupo volcano and Okataina volcanoes. 

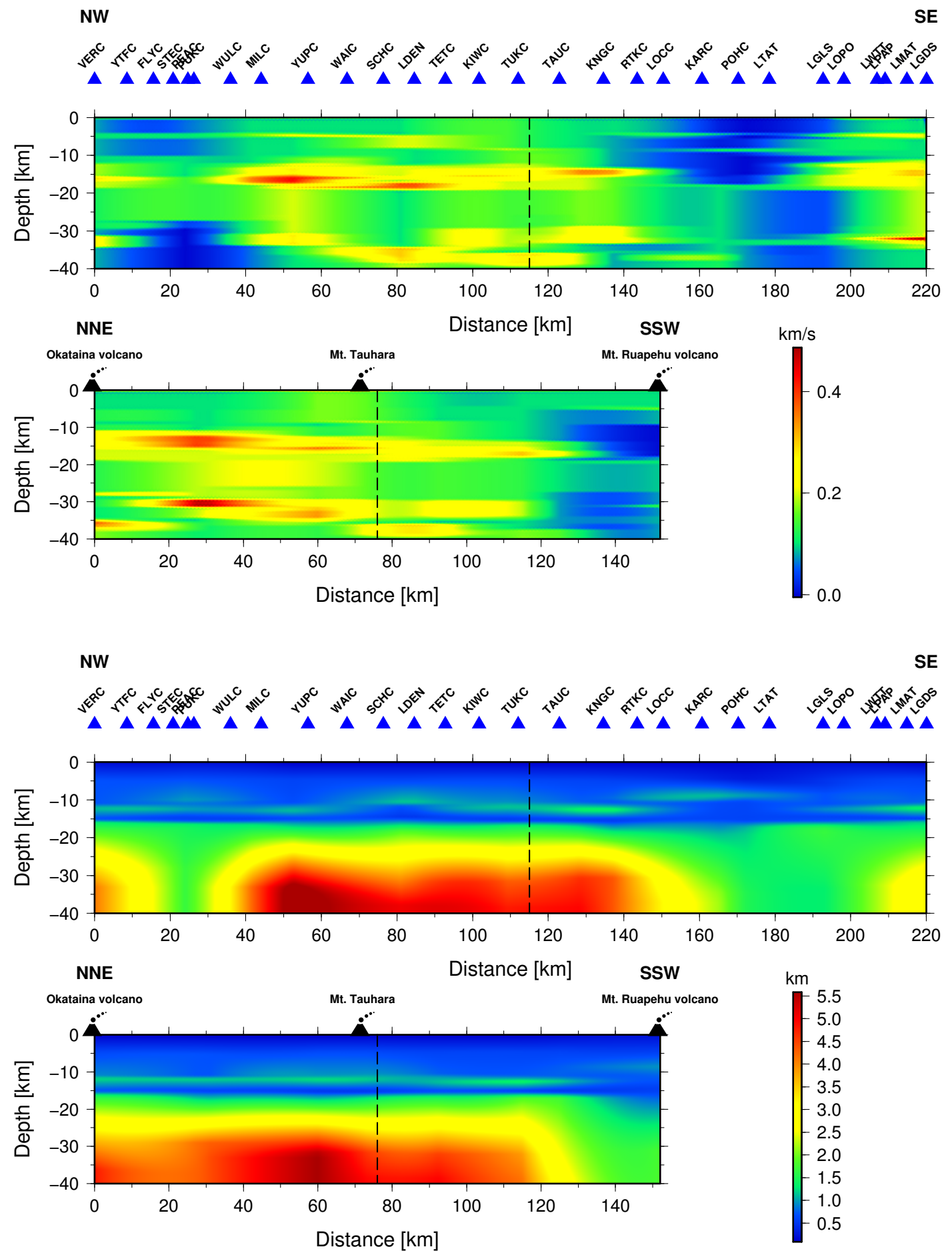

Figure 5.26 Standard deviation of the S-velocity (upper two panels) and depth (lower two panels) at each point of the 2D sections shown in Figure 5.23 and Figure 5.24. We calculated the histogram of the Svelocity at depth intervals of $0.5 \mathrm{~km}$ for the 1000 best fitting models of each $1 \mathrm{D}$ inversion. We then fitted a Gaussian curve to every histogram and defined one standard deviation to be the measure of variability. For the depth uncertainty we followed a similar approach, calculating the standard deviation for each layer, as defined in the parameterisation, and then interpolating these variances to depth intervals of $0.5 \mathrm{~km}$. It therefore expresses the range of different depths for each of the five layers above the half space (cf. Section 5.5.1) for the 1000 best-fitting models. 


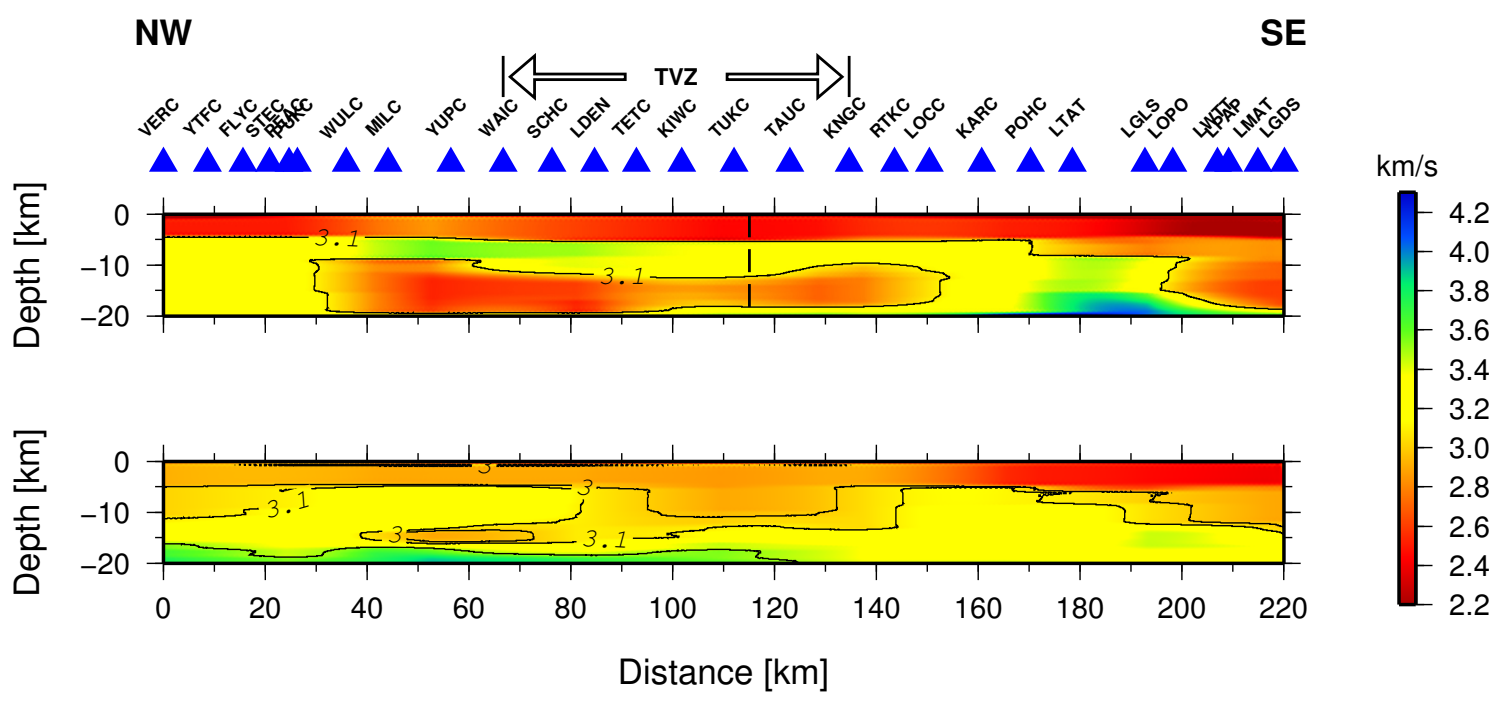

Figure 5.27 S-velocity vs. depth profile beneath the CNIPSE/WCNIPSE line (see Figure 5.1). The upper panel is a vertical section through the 3D S-velocity model calculated from Love wave maps between 6 and $11 \mathrm{~s}$ period. The lower panel shows the uppermost $20 \mathrm{~km}$ of the S-velocity model for the same profile calculated from Rayleigh wave maps between 5 and 25 s period (Figure 5.23).

Another obvious difference are the faster velocities beneath Mt. Ruapehu in the Love wave model. The velocities in the upper $5 \mathrm{~km}$ are in both profiles a lot slower in the Love wave model than in the Rayleigh wave model.

The S-velocity model inferred from Love wave phase velocities (Figure 5.29) exhibits much higher velocities throughout the mid and upper crust than the two previously discussed models. In the NNE-SSW profile, S-velocities below $15 \mathrm{~km}$ depth are in some areas as fast as $4.2 \mathrm{~km} / \mathrm{s}$. The NW-SE profile also displays very fast velocities of $\sim 3.7 \mathrm{~km} / \mathrm{s}$ and shows only little lateral variation. As the crust beneath the TVZ is most likely heterogeneous and contains melts and fluids, which decrease S-velocities rather than increase them, the S-velocity model from Love wave phase velocities does not seem physically reasonable and phase velocities appear to be consistently too fast. Surface wave maps for Love wave phase and group velocities are consistent with those for Rayleigh wave phase and group velocities (see Figure 5.15 and 5.16) which suggests that only a constant phase shift of the Love wave phase can be responsible for the fast Love wave phase velocities. Yao and Van Der Hilst [2009] showed that inhomogeneous noise source distributions can cause a phase shift of the cross-correlation functions and the resulting dispersion curves. Whether this can account for the velocity changes observed requires further investigation and is beyond the scope of this work. 


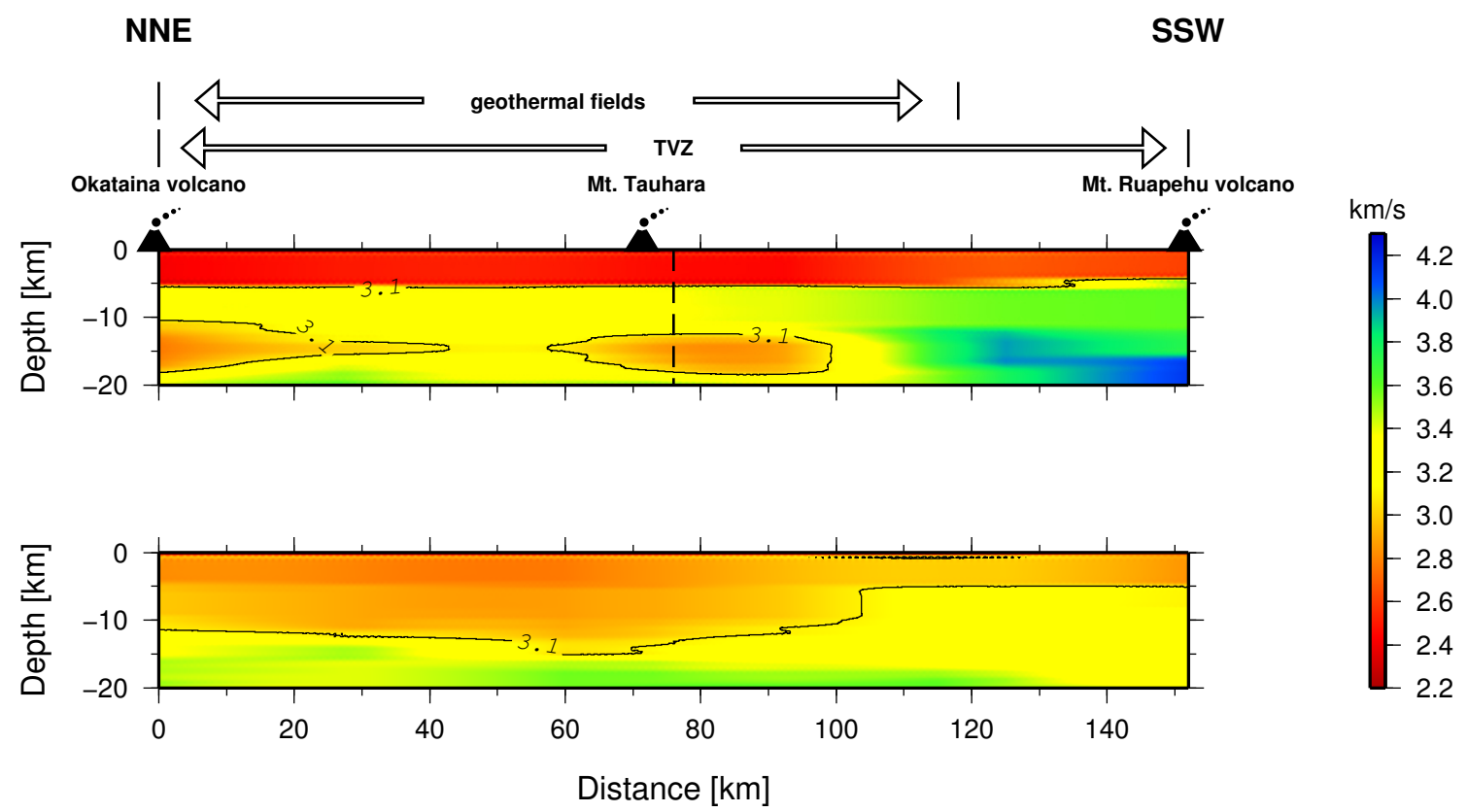

Figure 5.28 S-velocity vs. depth profile along the strike of the TVZ between the Okataina volcanoes and Mt. Ruapehu volcano (see Figure 5.1). The upper panel is a vertical section through the 3D S-velocity model calculated from Love wave maps between 6 and $11 \mathrm{~s}$ period. The lower panel shows the uppermost $20 \mathrm{~km}$ of the S-velocity model for the same profile calculated from Rayleigh wave maps between 5 and 25 s period (Figure 5.24).
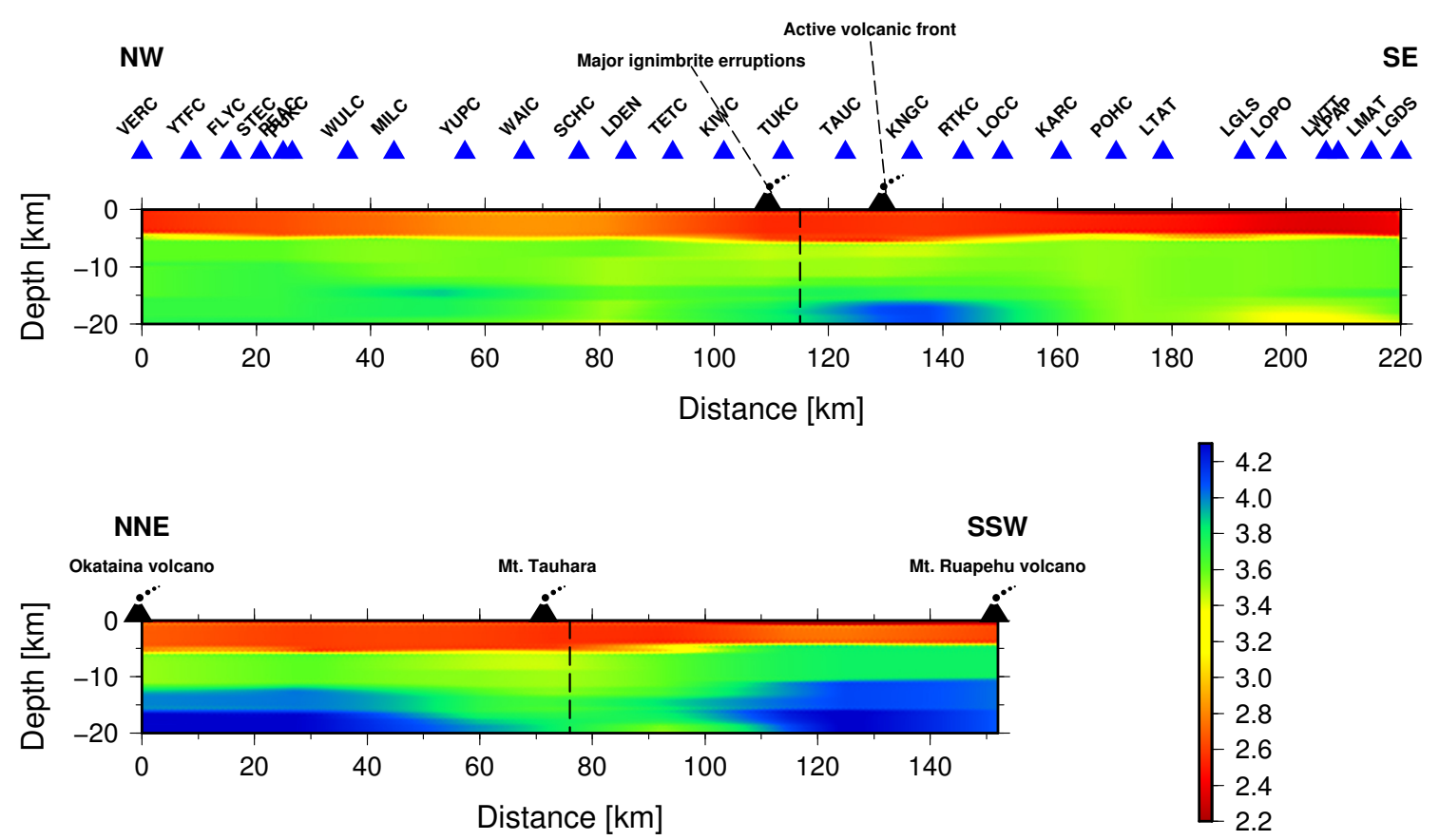

Figure 5.29 The upper panel is a vertical section along the WCNIPSE/CNIPSE line through the 3D Svelocity model calculated from Love wave phase velocity maps between 6 and $11 \mathrm{~s}$ period. The lower panel is a vertical section along the strike of the TVZ through the same 3D S-velocity model. 


\subsection{Discussion}

\subsubsection{Implications for the presence of melt}

Changes in S-velocity are mainly caused by a change of a medium's shear modulus. In a regime of extension, we expect melts and fluids to be one of the main causes of reduced shear modulus and we interpret the low velocities underneath the TVZ at $\sim 5-15$ $\mathrm{km}$, as shown in Figure 5.23 and 5.24, to be indicators of melt in the upper crust. Low $\mathrm{S}$-velocities in the uppermost layer beneath the TVZ are more likely due to the $2-3 \mathrm{~km}$ thick volcanic deposits [Stern et al., 1987]. Petrological studies inferred the source region of large rhyolitic eruptions to $\sim 3.5-8 \mathrm{~km}$ depth [Liu et al., 2005; Shane et al., 2008]. Wilson [2006] pointed out that, based on mineralogical compositions of eruptives, these shallow magma chambers might only be transient features which get fed by deeper lying zones ( $\sim 15 \mathrm{~km}$ depth) of partly or wholly crystallized silicic plutonic assimilations during periods of mafic intrusions from the lower crust. The depth ranges of these models are consistent with the zones of decreased S-velocity in our models. The numerous geothermal fields in the TVZ (see Figure 5.24 for their extent along the strike of the TVZ), and their related fluids are another possible cause for reduction of the shear-modulus. However the maximum penetration depth of geothermal fluids in the TVZ is estimated to be 5-8 km [Bibby et al., 1995] and therefore can only account for a part of the S-velocity reduction in our model. Li et al. [2003] showed that a thin layer of aqueous fluids above a thicker layer of partial melt could best explain high conductivity and high $\mathrm{Vp} / \mathrm{Vs}$ ratios in the crust below $\sim 15 \mathrm{~km}$ depth beneath the southeastern Tibetan Plateau. Fluids originating from phase transitions in the subducted plate and from crystallization processes in the overlying mantle and crust are likely to be present beneath the TVZ. From our surface wave analysis alone we cannot determine whether the inferred slow shear velocities are due to the presents of melts or aqueous fluids. Considering the tectonic regime a combination of both seems the most likely scenario. High heat flow at the surface $\left(\sim 700 \mathrm{~mW} / \mathrm{m}^{2}\right)$ and a shallow seismogenic zone beneath the central TVZ $(<8 \mathrm{~km})$ are indicators for temperatures high enough to melt water-saturated rocks at upper to mid-crustal depths [Lebedev and Khitarov, 1964; Bibby et al., 1995; Sibson and Rowland, 2003].

Assuming a constant density, we can calculate the amount of partial melt in the crust beneath the TVZ in relation to the inclusion geometry by employing results of the numerical modelling undertaken by Schmeling [1985]. As mentioned previously, relative shear velocity changes are better constrained by the surface wave inversion than absolute shear velocities. We therefore calculated the percentage of partial melt due to a velocity 


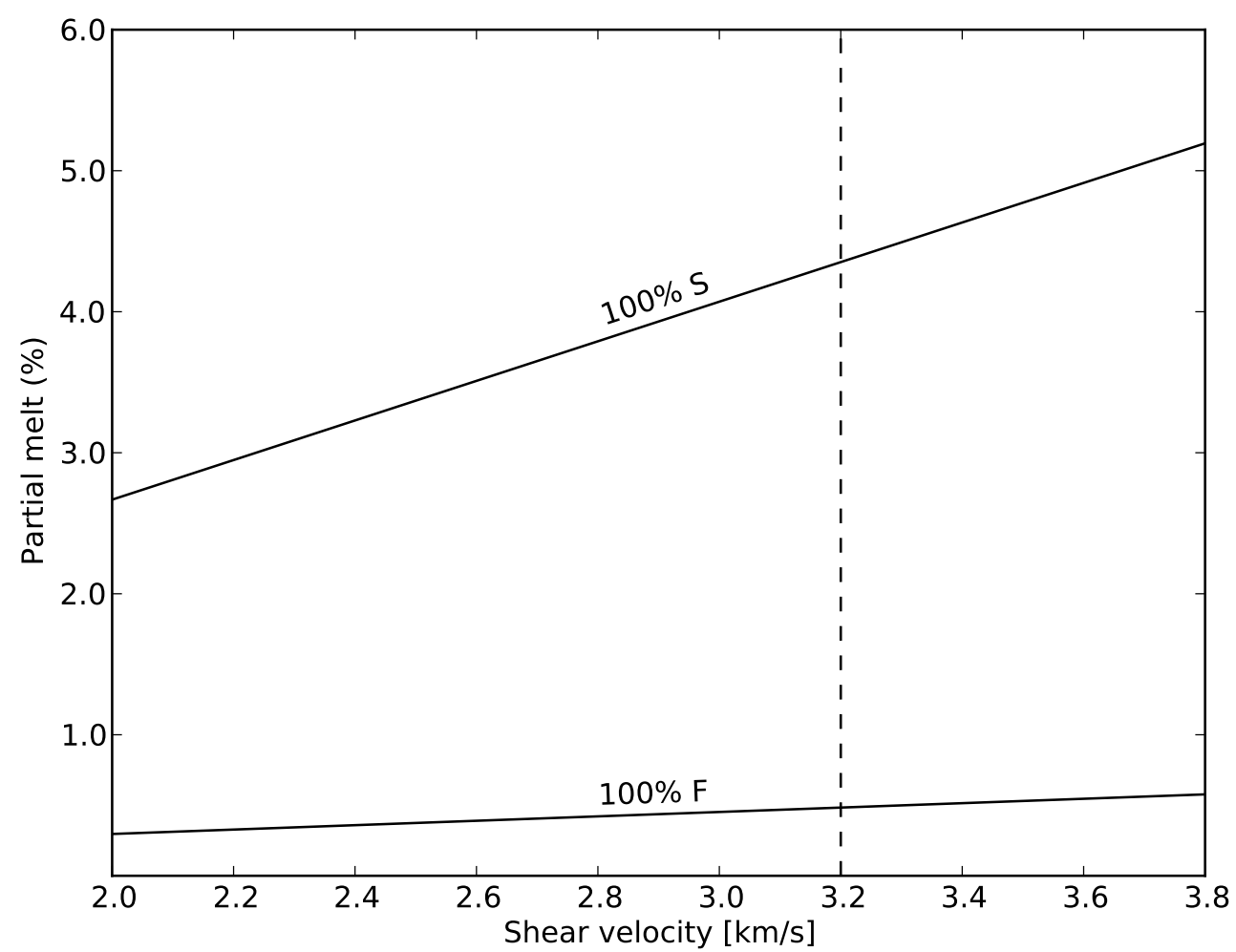

Figure 5.30 The percentage of partial melt depending on the inclusion geometry for a velocity change of $0.2 \mathrm{~km} / \mathrm{s}$, absolute velocities between 2.0 and $3.8 \mathrm{~km} / \mathrm{s}$ and a constant density of $2600 \mathrm{~kg} / \mathrm{m}^{3}$. The inclusion geometry is described by a superposition of melt films (F) and spherical inclusions. ' $100 \%$ F' means $100 \%$ of the inclusions are melt films and ' $100 \%$ S' stands for $100 \%$ of the inclusion having spherical shapes [Schmeling, 1985].

change of $0.2 \mathrm{~km} / \mathrm{s}$, as inferred for the crust beneath the TVZ, and different absolute velocity values, in relation to the inclusion geometry. The results are shown in Figure 5.30 for velocities varying between 2.0 and $3.8 \mathrm{~km} / \mathrm{s}$, a constant density of $2600 \mathrm{~kg} / \mathrm{m}^{3}$ and a generalized inclusion geometry described by the superposition of melt films and spherical inclusions [Schmeling, 1985]. The results corresponding to our observations lie along the dashed line in Figure 5.30. If all melt inclusions were present in the form of melt films, we would estimate the percentage of partial melt of less than $0.5 \%$ and, in contrast, if all inclusions were spherical the observed velocity change would correspond to more than $4 \%$ of partial melt.

Our estimate of $0.5-4 \%$ melt in the upper crust is compatible with the findings of Heise et al. [2007], who explained low-resistivity values at depths greater than $10 \mathrm{~km}$ beneath the central TVZ, inferred from a magnetotelluric profile subparallel to the CNIPSE/WCNIPSE line, in terms of the presence of $<4 \%$ interconnected partial melt. 
In a 3D magnetotelluric study of the central TVZ, Heise et al. [2010] later observed a number of highly conductive regions in the upper crust which they attributed to the presence of melt reservoirs with melt fractions of $\sim 50 \%$. The size of the bodies (diameters of $\sim 10-20 \mathrm{~km}$ ) is in general too small to be imaged by our surface wave inversion, but Heise et al. [2010] observed one of the highest conductivities beneath the Maroa volcanic centre which coincides with the topographic low we observe for the $3.1 \mathrm{~km} / \mathrm{s}$ isosurface shown in Figure 5.25. Another zone of high conductivity was identified by Heise et al. [2010] beneath the Mangakino volcanic centre between $\sim 15-22 \mathrm{~km}$ depth, and this corresponds well to the low-velocity zone we observe beneath stations SCHC and MILC (Figure 5.23). Furthermore the region of extensional faulting between Okataina volcanic centre and Maroa volcanic centre was described by Heise et al. [2010] as highly conductive between 10 and $20 \mathrm{~km}$ depth. The S-velocity model from surface waves shows slow velocities beneath the same region extending from the surface down to $\sim 15 \mathrm{~km}$ depth. One has to bear in mind that the same area coincides with high variability in the S-wave model (top panel in Figure 5.26) which means that a wider range of models fits the data equally well in this area and the model is therefore less well constrained than the low velocities beneath the central TVZ.

The difference in depth between the magnetotelluric and the Rayleigh wave inversion likely stems from the low resolution at shallow depths of Rayleigh waves at periods which are sensitive to structures at $\sim 10-20 \mathrm{~km}$ depth [Wathelet, 2005]. Due to the restriction in layer thickness imposed on the 1D S-velocity models by the a priori parameterisation (see Section 5.22), velocity changes are only likely to be observed at certain depth ranges. This exacerbates the limited depth resolution inherent to surface waves but is necessary to keep the parameter space as small as possible and the inversion process stable (i.e. repeatable). While we endeavour to perform the inversion as objectively as possible, the choice of the a priori search range for the 1D surface wave inversion as well as the choice of damping in the inversion for lateral velocity distributions both require the inversion results to be interpreted in conjunction with the knowledge of known geological features and the results of previous studies.

The LVZ in the S-velocity model inferred from Love wave group velocities shows an even stronger correlation with highly conductive areas in the northwest-southeast striking profile. Figure 5.31 shows the comparison between the profiles inferred from Rayleigh waves (Figure 5.31c), the profile inferred from Love wave group velocities (Figure 5.31a) and a 2D cross-section from the 3D conductivity model of Heise et al. [2010] (Figure $5.31 \mathrm{~b}$ ). The magnetotelluric cross-section is located approximately $10 \mathrm{~km}$ northeast of and subparallel to the CNIPSE/WCNIPSE line (Figure 5.32). The extent of the LVZ 


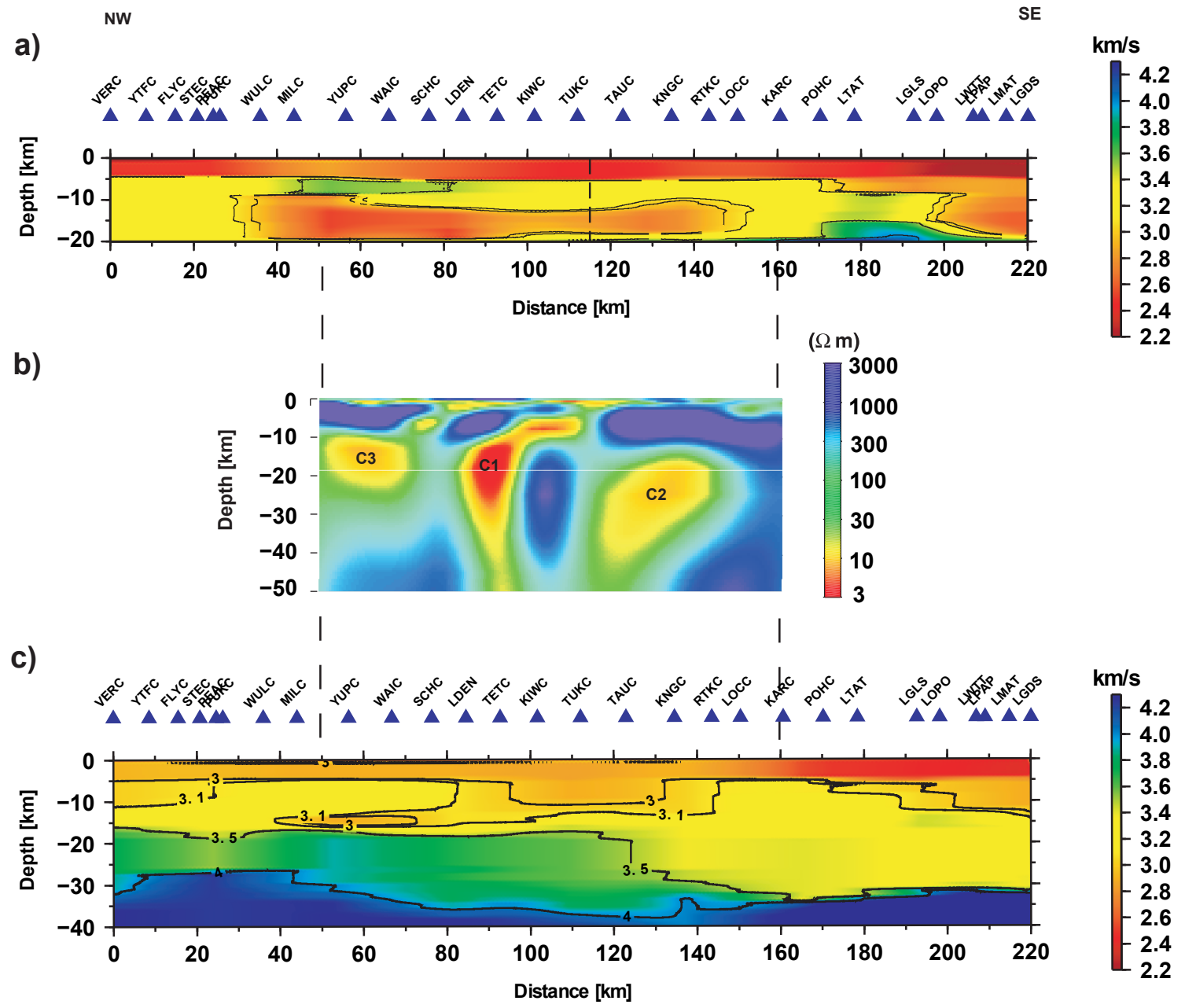

Figure 5.31 Figure shows the S-velocity model inferred from Love wave group velocities from Figure 5.27 (a), a 2D section through the 3D conductivity model of Heise et al. [2010] (b), and the S-velocity model along the CNIPSE/WCNIPSE line from Figure 5.23 inferred from Rayleigh wave phase and group velocities (c). Low velocities in both S-velocity models in the mid and upper crust correspond well to bodies of high conductivity (C1, C2, C3) described by Heise et al. [2010]. 


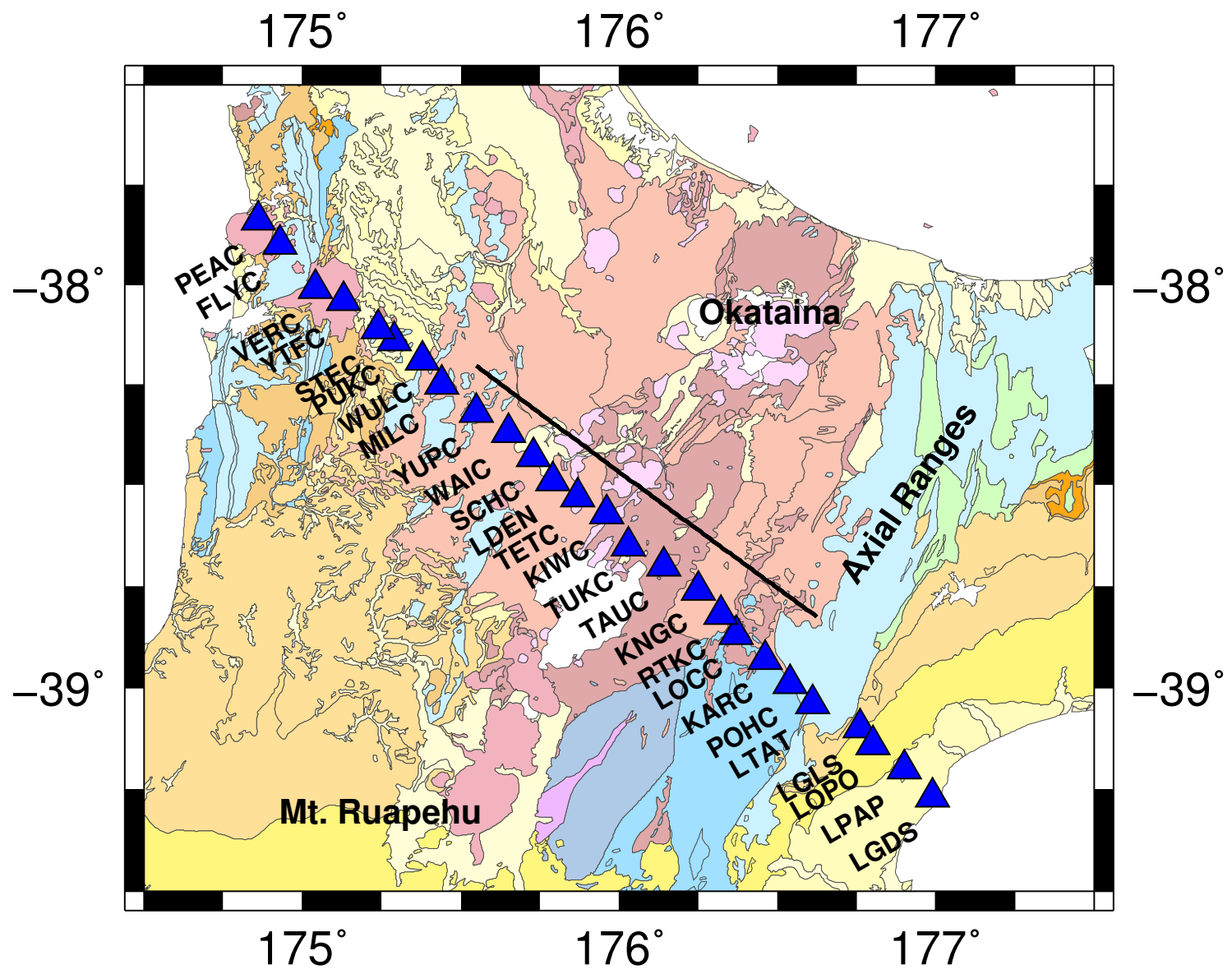

Figure 5.32 Location of the S-velocity profile and magnetotelluric Profile.The black line marks the location of the cross-section through the 3D conductivity model of Heise et al. [2010] (see Figure 5.31). 
along the strike of the profile and the depth on which it is centered match the locations of highly conductive bodies C1, C2 and C3 in the study of Heise et al. [2010, Figure 5.31b]. Lateral smearing in the 2D Love wave group velocity maps could cause the LVZ to appear as one body rather than the additional features revealed by the magnetotelluric study. Bannister et al. [2007] also inferred a low-velocity zone at a similar depth range beneath the stations YUPC, WAIC, SCHC and LDEN from the inversion of teleseismic Receiver functions. Robinson et al. [1981] observed an increase in relative travel-time residuals and P-to-S amplitude ratios from east to west along a $\sim 100 \mathrm{~km}$ long profile across the central TVZ subparallel to the WCNIPSE/CNIPSE line. Although Robinson et al. [1981] preferred a zone of low P-velocities and high attenuation in the mantle as an explanation for their observations, their results are also consistent with our model of low crustal S-velocities extending beneath and to the west of the TVZ. From magnetotelluric data Björnsson et al. [2005] inferred $\sim 3-10 \mathrm{~km}$ as the depth to the magma chambers feeding the silicic volcanism in central Iceland, a depth range shallower than observed in this study from the Love wave inversion and by Heise et al. [2010] but similar to the results from the Rayleigh wave inversion. Inverting Rayleigh wave dispersion curves from ambient seismic noise and earthquake data, Stachnik et al. [2008] found zones of low S-velocity beneath the calderas of Yellowstone in the upper $\sim 15 \mathrm{~km}$ underlain by structures of velocities between 3.4 and $3.8 \mathrm{~km} / \mathrm{s}$. They interpreted the faster velocities as basaltic magma originating from the upper mantle which reaches neutral buoyancy at $\sim 15-20 \mathrm{~km}$ depth where it produces rhyolitic magma by remelting of older basaltic intrusives, fractionation and melting of the surrounding upper-crustal rocks. This is quite similar to the scale and petrology assumed for the evolution of rhyolitic volcanism in the central TVZ and is consistent with our S-velocity models, as we discuss in the following section. Another study involving surface wave information from ambient seismic noise [Nishida et al., 2008] showed slow S-velocities beneath andesitic volcanoes in the Japanese regions of Tohoku, Chubu and Kanto.

The faster S-velocities beneath Mt. Ruapehu in the Love wave model compared to the Rayleigh wave model (Figure 5.28) may be artifacts of lateral smearing. As we used only temporary stations in our Love wave analysis, Mt. Ruapehu lies at the border of the region of good path coverage and therefore good resolution (Figure 5.15; black line). In contrast Mt. Ruapehu lies well within the region of good resolution for Rayleigh waves. Lateral smearing of velocities from within the region of high resolution into regions of poor resolution might therefore cause Love wave group velocities to appear faster than their true value in the area around Mt. Ruapehu.

A possible explanation for the difference in depth of low crustal velocities beneath the 
TVZ between Rayleigh and Love wave S-velocity models is given by the difference of sensitivity kernels for either wave type (Figure 5.18). Rayleigh waves at periods which are sensitive to structures at 10-20 km depth have only little sensitivity to shallower structures. In contrast, Love waves at these periods have even higher sensitivity at shallower depths. The use of both Rayleigh wave phase and group velocities should ameliorate this effect. A joint inversion of Rayleigh and Love waves did not result in satisfactory dispersion curve fits to either wave type. Similar observations were made by Shapiro et al. [2004] and Moschetti et al. [2010] and explained by both in terms of crustal radial anisotropy. A simple comparison of our Love and Rayleigh wave models to estimate the degree of radial anisotropy, as done by Behr et al. [2010] for 1D S-velocity models inferred from Rayleigh and Love wave velocities, is likely to show differences in S-velocity structure rather than radial anisotropy. This emphasizes the need to include radial anisotropy as an additional parameter into the inversion process in order to jointly invert Rayleigh and Love wave velocity measurements.

\subsubsection{Implications for TVZ evolution}

Comparing the S-velocity profile along the WCNIPSE/CNIPSE line inferred from Rayleigh waves to P-velocity profiles from active source studies conducted by Stern and Benson [2011] and Stratford and Stern [2006] and to active and passive source studies by Harrison and White [2006] and Harrison and White [2004] (Figure 5.33), it is evident that the change to fast lower crustal velocities below $\sim 15 \mathrm{~km}$ beneath the WCNIPSE/CNIPSE line is a common feature of all three studies. S-velocities at this depth lie between 3.5 and $3.7 \mathrm{~km} / \mathrm{s}$ and P-velocities between $\sim 6.8$ and $7 \mathrm{~km} / \mathrm{s}$. It is further evident that higher S-velocities extend to shallower depths in the northwest of the profiles than in the southeast. This agrees with shallow Moho depths of $\sim 25 \mathrm{~km}$ observed in the western and northwestern North Island [Stern et al., 2006; Horspool et al., 2006; Behr et al., 2010] and a deeper Moho beneath the eastern North Island [Reyners et al., 1999].

As noted in the Introduction, in connection with the rhyolitic volcanism, the observed extension rates of the central TVZ and the tectonic uplift of the central North Island, two different mechanisms have been proposed to explain the fast P-velocities in the lower crust beneath the central TVZ. Harrison and White [2006] and Rowland et al. [2010] preferred a model in which the thickness of the crust beneath the central TVZ does not change and extension is accommodated instead by mafic dyke intrusions and magma migration from the mantle into the lower and middle crust, occasionally injecting the shallow magma chambers in the upper crust, which are the inferred sources for the rhyolitic volcanism 


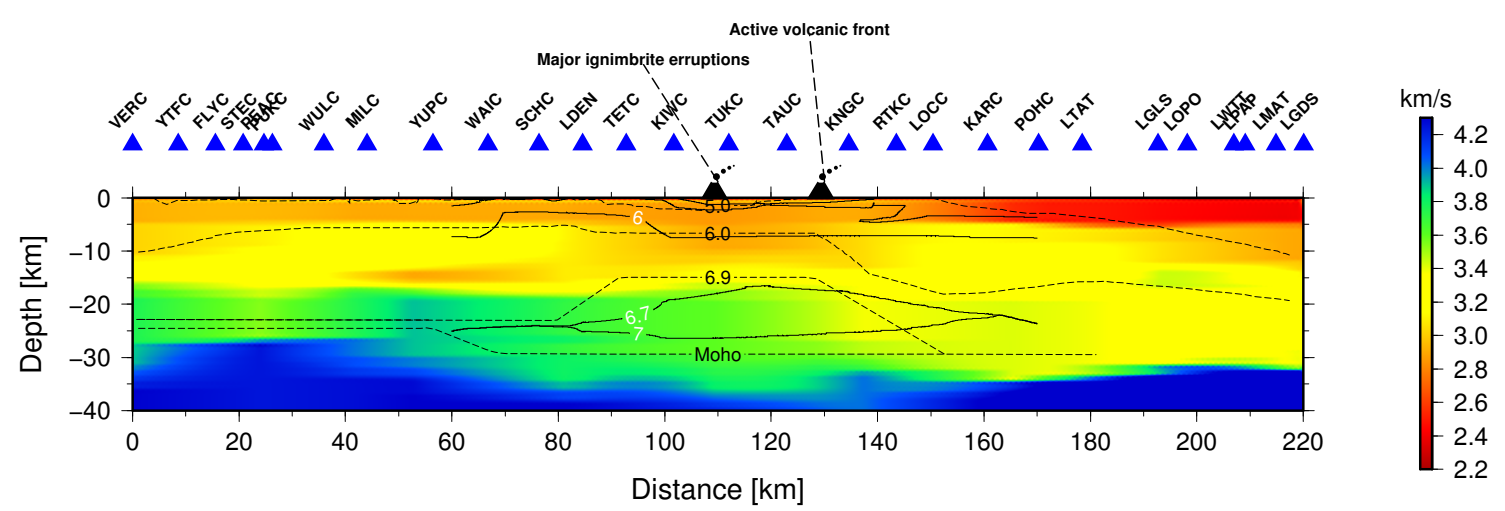

Figure 5.33 Comparison of the Rayleigh wave S-velocity profile along the CNIPSE/WCNIPSE line (Figure 5.23) with the results from active source studies done by Stern and Benson [2011] (solid lines; white annotations) and Harrison and White [2006] (dashed lines;black annotations).

in the central TVZ [Wilson, 2006; Liu et al., 2005; Shane et al., 2008]. In this model, the uplift of the central North Island is caused by a low-density mantle which has been depleted of its mafic components. The same mechanism was proposed by Takahashi et al. [2008] to explain high lower crustal P-velocities observed beneath the Mariana Trough, a region of back-arc extension in the Izu-Bonin-Mariana arc system southeast of Japan.

An alternative model, proposed by Pulford and Stern [2004], Stratford and Stern [2006], Stern et al. [2006] and Stern and Benson [2011], suggested that lower-crustal and upper mantle lithosphere beneath the central and western North Island has been replaced by less dense, high-velocity and hot asthenospheric material during a delamination process prior and coeval to the onset of back-arc extension in the TVZ. Underplating then forms a mafic and hot lower crust with high percentages of partial melt, which differentiates over time with felsic magma migrating into the mid and upper crust, leaving behind a mafic restite depleted of its felsic components. The observed uplift of the central North Island is attributed in this model to the hot and therefore buoyant asthenospheric material emplaced during the delamination process. A similar mechanism whereby mafic lowercrustal material is delaminated after being depleted of its felsic components during partial melting, was put forward by Tatsumi [2005] as an explanation of the high lower crustal velocities and andesitic volcanism observed in back-arc settings.

Our S-velocity model does not explicitly favour one model over the other, although the extent of the faster S-velocities to the west of the TVZ beneath the WCNIPSE/CNIPSE line at depths greater than $\sim 15 \mathrm{~km}$ (Figure 5.23) suggests that the wavelength of the mechanism underlying the S-velocity increase is longer than the width of the TVZ. The process proposed by Harrison and White [2006] and Rowland et al. [2010] is linked to the extension of the TVZ and should therefore be constrained to a region beneath the TVZ. 
Both models are in accordance with the petrological model for the evolution of rhyolitic magma beneath the central TVZ [Wilson, 2006]. However their different lateral extent implies a difference in wavelength of the uplift of the central North Island.

Although we observe an increase of $\mathrm{S}$-velocities below $\sim 15 \mathrm{~km}$, absolute $\mathrm{S}$-velocities of $\sim 3.4-3.7 \mathrm{~km} / \mathrm{s}$ are still slow compared to more standard lower-crustal S-velocities of $\sim 3.9 \mathrm{~km} / \mathrm{s}$ [PREM, Dziewonski and Anderson, 1981]. Combined with P-velocities of $6.8-7.0 \mathrm{~km} / \mathrm{s}$ at similar depth ranges this results in Poisson's ratios of 0.29-0.35. Since Svelocities are inferred from smooth surface wave maps, their absolute values are likely to deviate from the true S-velocity and therefore the Poisson's ratios inferred here represent only an estimate of upper and lower bounds. If the actual Poisson's ratios lie within these bounds, they indicate the presence of fluids and melts in the lower crust, which is in accordance with the two models of crustal evolution beneath the central TVZ described above.

\subsection{Conclusion}

We have successfully inferred the first pseudo-3D crustal S-velocity structure for the central North Island of New Zealand. The main features of the S-velocity model correlate well with 2D cross-sections of previous magnetotelluric and active source studies and the inferred lower crustal structure is similar to that observed in a 3D passive source study. This study has two ramifications for studies of active continental rifts elsewhere. First, our TVZ observations support existing volcanological models of the production and migration of rhyolitic melts in continental rift zones generally. Second, the pervasiveness of seismic noise offers clear potential for continuous or repeated tomographic imaging of active rifts and the evaluation of volcanological models.

In order to look deeper into the mantle, dispersion curves from ambient noise crosscorrelations need to be augmented by dispersion curves from earthquakes, as the shape of New Zealand and thereby the geometry of possible onshore seismic network layouts precludes the inference of reliable surface wave maps at periods longer than $\sim 25 \mathrm{~s}$. 
5. Ambient noise tomography of the central North Island 


\section{Beamforming and slant stack analysis of ambient noise data for noisefield and structural characterisation}

In this chapter, we expand on the topic of inferring noise directionality from plane wave beamforming, which we briefly introduced in Section 5.2. Characterizing the ambient noise climate is important for understanding noise cross-correlation results. Knowing the connection between noise sources and noise cross-correlation can potentially help to unravel noise source characteristics from seismic noise recordings. In addition to examining noise directionality, it can be useful to extract average surface wave dispersion curves from the ambient noise field across a whole array. Average dispersion curves can be used as reference curves when measuring phase velocities from noise cross-correlations between station pairs, and to infer a reference 1D S-velocity profile beneath the array, as we will show in Section 6.2.1 and 6.2.2. Results from plane wave beamforming of seismic noise records from two local broadband deployments, one around Mt. Taranaki on the western North Island and the other one around Mt. Tongariro and Mt. Ruapehu in the central North Island, indicate seismic source regions compatible with high near-coastal ocean wave heights taken from a wave action model for New Zealand. Both slant stacking and plane wave beamforming further reveal the presence of higher-order Rayleigh waves in the seismic noise field in addition to fundamental mode Rayleigh waves.

\subsection{Introduction}

Beamforming and related array techniques have been applied to ambient seismic noise recordings in several studies ranging from small-aperture arrays [e.g. Di Giulio et al., 2006] to arrays spanning several hundreds of kilometers [e.g. Harmon et al., 2008]. Plane 
wave beamforming is especially suitable for inferring the backazimuth and average dispersion curves of surface waves arriving from distant sources. The dispersion information can then be used to calculate an average S-velocity structure beneath the sensor array. Average dispersion curves can also be measured from slant stacking [Chapman, 1978] of ambient noise cross-correlations, which is similar to the beamforming method, but has been shown to have better resolution in the case of directional noise arriving from a small backazimuth range [Gouédard et al., 2008]. A further use of average dispersion curves is to determine the correct number of cycles when measuring phase velocities from noise cross-correlation functions (see Section 2.3.2).

It has been known for some time that the primary and secondary microseisms result from interaction between ocean swells and atmospheric pressure fluctuations with the Earth's surface [e.g. Longuet-Higgins, 1950; Friedrich et al., 1998; Bonnefoy-Claudet et al., 2006; Snieder and Wapenaar, 2010; Kibblewhite and Ewans, 1985]. Nevertheless the microseisms' source regions, the specific locations at which most of the energy is generated, remain debated. For example, by examining amplitude asymmetries between the causal and acausal halves of noise cross-correlation functions, which are thought to be produced by asymmetries in the underlying noise source distribution [e.g. Larose et al., 2006], Stehly et al. [2006] proposed that the secondary microseism is generated at coastlines and that the primary microseism is created in deeper parts of the ocean. Kedar et al. [2008] could relate seismic observations of microseisms in North America, Greenland, Iceland and Europe to a source region in the North Atlantic by modelling ground displacements resulting from wave-wave interactions in the deep sea and comparing them to seismic records. Rhie and Romanowicz [2006] observed high correlations in time between amplitude spectra of significant wave height at near-coastal ocean buoys and those from onshore seismic stations in southern California and Japan. Both timeseries were coincident with a large storm coming from the Pacific Ocean and impacting on southern California's coastline. This led them to conclude that long-period (the earth's "hum" at 240 s) and short period seismic noise (primary and secondary microseisms) were produced near coastlines. Employing similar techniques as Stehly et al. [2006], Yang and Ritzwoller [2008] demonstrated coupling of the source regions for primary and secondary microseisms, and instead attributed azimuthal variations in source backazimuths for the primary and secondary microseisms to wave propagation effects associated with differences in wavelength between the two spectral peaks.

Understanding the distribution of ambient noise sources is of particular importance when establishing the causal relationship between noise sources and their cross-correlation functions. As mentioned in Chapter 3.5, theoretical constraints on the distribution of 
noise sources required to construct the Green's function between two seismometers from ambient noise cross-correlations, are at best only approximated in most network environments. Although several studies have explored the possibility of extracting components of the Green's function in the case of azimuthally heterogeneous noise source distributions [Larose et al., 2006; Wapenaar, 2006; Tsai, 2009; Yao and Van Der Hilst, 2009, e.g.], the conclusive link between noise source distribution and the Green's function still remains to be established. As Yang and Ritzwoller [2008] pointed out, this link also incorporates a medium's elastic and inelastic properties between the noise source and the recording seismometer. Consequently the causal relationship between noise source distribution and the noise cross-correlation functions has to be established separately for every study area.

As an initial step in this direction we infer the noise climate in the central North Island of New Zealand within the period range of the secondary microseism from plane wave beamforming results. This extends the study of Brooks et al. [2009a] who used the same method to study the ambient seismic noise field around Mt. Taranaki (Figure 6.1) and whose results we summarize in Section 6.2.1. We further demonstrate that multimode surface wave information obtained from slant stacking of virtual common shot gathers of ambient noise cross-correlations is consistent with results from plane wave beamforming.

\subsubsection{Plane wave beamforming}

The most common form of beamforming is delay-and-sum beamforming [Johnson and Dudgeon, 1993], in which seismograms recorded at each seismometer in an array are shifted in time to align them with plane waves from a presumed source backazimuth, and are then stacked to form the "beam". When this is done in a grid-search over a range of backazimuths and slowness values, the maximum amplitude of the beam corresponds to the slowness vector of the source.

In this study, we employ the beamforming method implemented by Brooks et al. [2009a], which involves first cutting each day-long trace into hour-long segments, downweighting the amplitude of each trace to half its standard deviation, (in order to reduce any earthquake signals), and then employing a frequency-domain beamforming algorithm. For an array of $M$ stations, the beamformer in the frequency domain can be described as follows [Johnson and Dudgeon, 1993]:

$$
Z(t, \omega)=\sum_{m=1}^{M} w_{m} Y_{m}(t, \omega) e^{j \vec{k} \cdot \vec{x}_{m}}=\mathbf{e}^{\dagger} \mathbf{W} \mathbf{Y}(t, \omega)
$$


Here $w_{m} Y_{m}(t, \omega)$ is the windowed Fourier Transform of the trace recorded at station $m$, and $e^{j \vec{k} \vec{x}_{m}}$ is the phase shift for station $m$, located at $\vec{x}_{m}$, and a plane wave with wave vector $\vec{k}$.

As indicated in Equation 6.1, the sum over all $m=1 \ldots M$ can be written in vector form with e being the vector of phase shifts at each station, $\dagger$ denoting complex conjugation, and WY being the vector of windowed Fourier Transforms for all $M$ stations. In this study, we focus on the beamformer's steered response power [Johnson and Dudgeon, 1993], which is defined as

$$
\begin{aligned}
P(\mathbf{e}, \omega) & =\mathbf{e}^{\dagger} \mathbf{W Y} \mathbf{Y}^{\dagger} \mathbf{W}^{\dagger} \mathbf{e} \\
& =\mathbf{e}^{\dagger} \mathbf{C e} .
\end{aligned}
$$

Since shifting a trace in time corresponds to a simple multiplication in the frequency domain, frequency-domain beamforming is considerably faster than time-domain beamforming. As beamforming of ambient seismic noise involves processing long durations (up to several months) of continuous seismic records, the processing speed is of particular importance.

An array of seismometers samples a wavefield in both time and space: as it samples only certain aspects of the wavefield, - depending on the seismometers' instrument characteristics - , the sampling frequency and the alignment of the seismometers, it can be described as a spatiotemporal filter [Johnson and Dudgeon, 1993]. Every array geometry therefore possesses a spatial Nyquist frequency determining which wavelengths can be resolved. For a linear array, the minimum resolvable wavelength is simply twice the minimum interstation distance, as a consequence of the requirement that at least two sampling points are needed to define a wavelength unambiguously [Gouédard et al., 2008]. Conversely, the maximum resolvable wavelength corresponds to twice the maximum station spacing, or in other words to the aperture of the array [Poggi and Fäh, 2010].

For a two-dimensional array with irregular station spacing there is generally no simple analytical expression for the array's resolution capabilities. It is therefore common practice to describe the array in terms of its array response, also known as the array pattern, which describes the beam resulting from a known source with known frequency and unit amplitude [Wathelet et al., 2008; Johnson and Dudgeon, 1993]. As mentioned above in Section 5.2, this is conceptually similar to the Point Spread Function used in astrophysics to describe the resolution capabilities of a telescope [Bertero and Boccacci, 2005].

It follows from the assumption of plane waves arriving from a noise source at a particular 
backazimuth that the waves arrive at every seismometer of an array from the same angle. Assuming spherical wave propagation instead, the backazimuth of the noise source differs at every seismometer. Following Johnson and Dudgeon [1993], the maximum angular error $\varepsilon$ of the inferred backazimuth arising due to the plane wave assumption can be estimated as:

$$
\varepsilon \approx \frac{R_{a}}{d}
$$

Here $R_{a}$ is the radius of an array's aperture and $d$ is the distance to the presumed source. This implies, that to ensure a plane wave approximation error of less than $1^{\circ}$, the source needs to be at a distance greater than 57 times the radius of the array's aperture.

\subsubsection{Slant stacking}

A set of symmetric noise cross-correlation functions (Section 2.3) between all possible station pairs of a seismic array, ordered according to their interstation distance, can be regarded as a common shot point gather. If the approximation of a layered velocity model beneath the array is justifiable, the traveltime between each station pair depends only on the distance between the two stations. The common shot point gather can then be converted to a slant stack [Chapman, 1978] by shifting the Fourier transformed correlation functions according to a presumed phase velocity value and then stacking the resulting traces. Varying the velocity value systematically results in a complex wavefield, in which each trace corresponds to the spectrum of all the cross-correlation functions, stacked for a certain velocity value. The real part of each trace will exhibit a maximum when the presumed velocity value corresponds to the phase velocity of a surface wave train of a certain frequency that is present in all of the correlation functions [Park, 1999].

McMechan and Yedlin [1981] proposed slant stacking as a form of wavefield transformation into the slowness-frequency domain for use with common shot gathers of active source studies in order to retrieve surface wave dispersion information. They demonstrated the slant stack's utility by retrieving the fundamental mode Rayleigh wave phase velocity dispersion curve from a marine active source dataset. Mokhtar et al. [1988] later employed this method to infer Rayleigh wave phase velocity dispersion curves from an onshore active source dataset and used these to infer S-velocity profiles of the Arabian Shield. In a New Zealand setting, Horspool et al. [2006] used slant stacking to infer fundamental mode Rayleigh wave dispersion curves from teleseismic earthquakes, which they inverted in combination with the results of a $\mathrm{P}$-wave receiver function study to obtain S-velocity profiles beneath the Northland Peninsula, New Zealand (cf. Chapter 4). 
Louie [2001] employed slant stacking to extract Rayleigh wave dispersion curves from ambient seismic noise recordings on a linear array of geophones. Applications of slant stacking to synthetic noise cross-correlation functions were first presented by [Gouédard et al., 2008], who successfully retrieved parts of the fundamental and first higher mode Rayleigh and Love wave phase velocity dispersion curves. Bussat et al. [2009] also employed this technique with noise cross-correlation functions calculated between pairs of instruments in an array of ocean-bottom seismometers to infer the Svelocity structure of the uppermost $5 \mathrm{~km}$ of the crust.

As with plane wave beamforming, aliasing can occur in slant stacking if the station spacing exceeds half the wavelength at the periods of interest. In a virtual common shot gather, such as the one described for noise correlation functions, the spacing between traces depends on the distribution of distances between stations, which will be irregular in a typical 2D network layout intended for earthquake analysis. Hence, the minimum resolvable wavelength is determined by the maximum distance between adjacent traces. In addition, the irregularity of the station spacing is likely to further reduce the wavelength below which aliasing occurs as shown in the following calculation. Following Park [1999], for a wavefield $u(x, t)$, with $x$ being the source-receiver distance, slant stacking can be expressed as follows:

$$
V(\omega, \phi)=\int e^{-i \Phi(x, \omega)} e^{i \phi x}\left[\frac{A(x, \omega)}{|A(x, \omega)|}\right] d x \approx \sum_{j=1}^{m} e^{-i \Phi\left(x_{j}, \omega\right)} e^{i \phi x_{j}}\left[\frac{A\left(x_{j}, \omega\right)}{\left|A\left(x_{j}, \omega\right)\right|}\right] .
$$

Here $A(x, \omega) e^{-i \Phi}$ is the Fourier transform of the wavefield $u(x, t)$ at distance $x$ and angular frequency $\omega, \phi$ is the slant stack operator, $\phi=\omega / c(\omega)$, with $c(\omega)$ the phase velocity at angular frequency $\omega$, and $m$ the number of source-receiver pairs.

For $m=2$, corresponding to two source-receiver pairs, $\operatorname{Re}(V)$ is maximized when $\Phi_{1}=$ $\phi x_{1}$ and $\Phi_{2}=\phi x_{2}$, where $\Phi_{i}=\Phi\left(x_{i}, \omega\right)$. This means that

$$
\Phi_{1}=\phi x_{1}=\frac{\omega}{c(\omega)} x_{1} \quad \text { and } \quad \Phi_{2}=\phi x_{2}=\frac{\omega}{c(\omega)} x_{2} .
$$

Omitting the explicit $\omega$ dependency of $c(\omega)$ for clarity, we can write

$$
\Phi_{1}-\Phi_{2}=\frac{\omega}{c}\left(x_{1}-x_{2}\right)
$$


and adding $2 \pi n(n \in \mathbb{N})$ to both sides results in

$$
\begin{aligned}
\Phi_{1}-\Phi_{2}+2 \pi n & =\frac{\omega}{c}\left(x_{1}-x_{2}\right)+2 \pi n \\
& =\frac{\omega}{c}(\Delta x)+2 \pi n \\
& =\frac{\omega}{c^{\prime}}(\Delta x) .
\end{aligned}
$$

since $e^{i\left(\Phi_{1}-\Phi_{2}+2 \pi n\right)}=e^{i\left(\Phi_{1}-\Phi_{2}\right)}$ we can rewrite equation 6.10 as

$$
\Phi_{1}-\Phi_{2}=\frac{\omega}{c^{\prime}}(\Delta x)
$$

Comparing equations 6.7 and equation 6.11 yields

$$
\frac{\omega}{c} \Delta x+2 \pi n=\frac{\omega}{c^{\prime}} \Delta x
$$

and hence

$$
c^{\prime}=\frac{1}{\frac{1}{c}+\frac{2 \pi n}{\omega \Delta x}} .
$$

This expresses the phase velocity $c^{\prime}$, at which aliasing occurs, depending on $\Delta x$. Therefore, it will vary, if $\Delta x$ differs between each pair of traces in the wavefield. If the various $\Delta x$ differ only slightly, aliasing will still be evident but reduced in amplitude compared to the amplitudes of the true dispersion curves.

The maximum observable wavelength of such a virtual common shot gather is more difficult to determine. Gouédard et al. [2008] used three times the maximum interstation distance, which seems on empirical grounds to be too broad (see Figure 7 of Gouédard et al. [2008]). One could argue that the minimum interstation distance restricts the maximum observable wavelength as stations need to be at least two wavelengths apart to avoid interference effects between the causal and acausal parts of the noise cross-correlation function (cf. Section 2.3). However, this also seems to be too restrictive, as the dispersion curves will be shown below to be clearly visible and to correlate well with beamforming results beyond this limit. We therefore restrict the dispersion curves from slant stacking to the period range for which the beamforming results are reliable [Brooks et al., 2009a]. 


\subsubsection{Datasets}

We apply plane wave beamforming and slant stacking to seismograms of ambient noise recorded during two temporary deployments: the Mt. Taranaki deployment spanning Mt. Taranaki volcano on the west coast of the North Island [Figure 6.1; Sherburn and White, 2005]; and the Seismic Tomography Around Ruapehu and Tongariro (START) deployment [Figure 6.2; Rowlands et al., 2005] around Mt. Tongariro and Mt. Ruapehu volcano in the central North Island. The Mt. Taranaki deployment consisted of 75 three-component broadband stations which were operating between December 2001 and September 2002. Data from this deployment could be recovered for 68 stations. During the START deployment, 28 three-component broadband stations were deployed for six months between January and June 2001.

\subsection{Results}

\subsubsection{Mt. Taranaki deployment}

\section{Multimode dispersion curves from plane wave beamforming (summary of Brooks et al., 2009a, b)}

Plane wave beamforming results for ambient noise recorded at the Mt. Taranaki deployment [Sherburn and White, 2005] during four southern hemisphere winter months in 2002 have been described previously [Brooks et al., 2009a, b]. For the sake of completeness, we paraphrase below some of the key results of that study.

Beamforming results within the frequency band spanning the secondary microseism for noise recordings from the Mt. Taranaki deployment during the southern hemisphere winter months of May-August 2002 reveal two signals traveling at velocities of $\sim 2-2.5 \mathrm{~km} / \mathrm{s}$ and $\sim 3-4.5 \mathrm{~km} / \mathrm{s}$. Both signals exhibit dispersive behaviour and can be related to the fundamental and first higher Rayleigh wave mode. Blue lines in Figure 6.3 illustrate the two signals for each of the four months. They are plotted on top of theoretical dispersion curves for the fundamental and the first two higher Rayleigh wave modes (grey lines in Figure 6.3) calculated from 1D Vp and Vs models for Mt. Taranaki inferred from local earthquake recordings and published by Sherburn and White [2005].

The lower-velocity signal from the beamformer output matches almost exactly the theoretical dispersion curve for the fundamental mode Rayleigh wave. The higher-velocity 


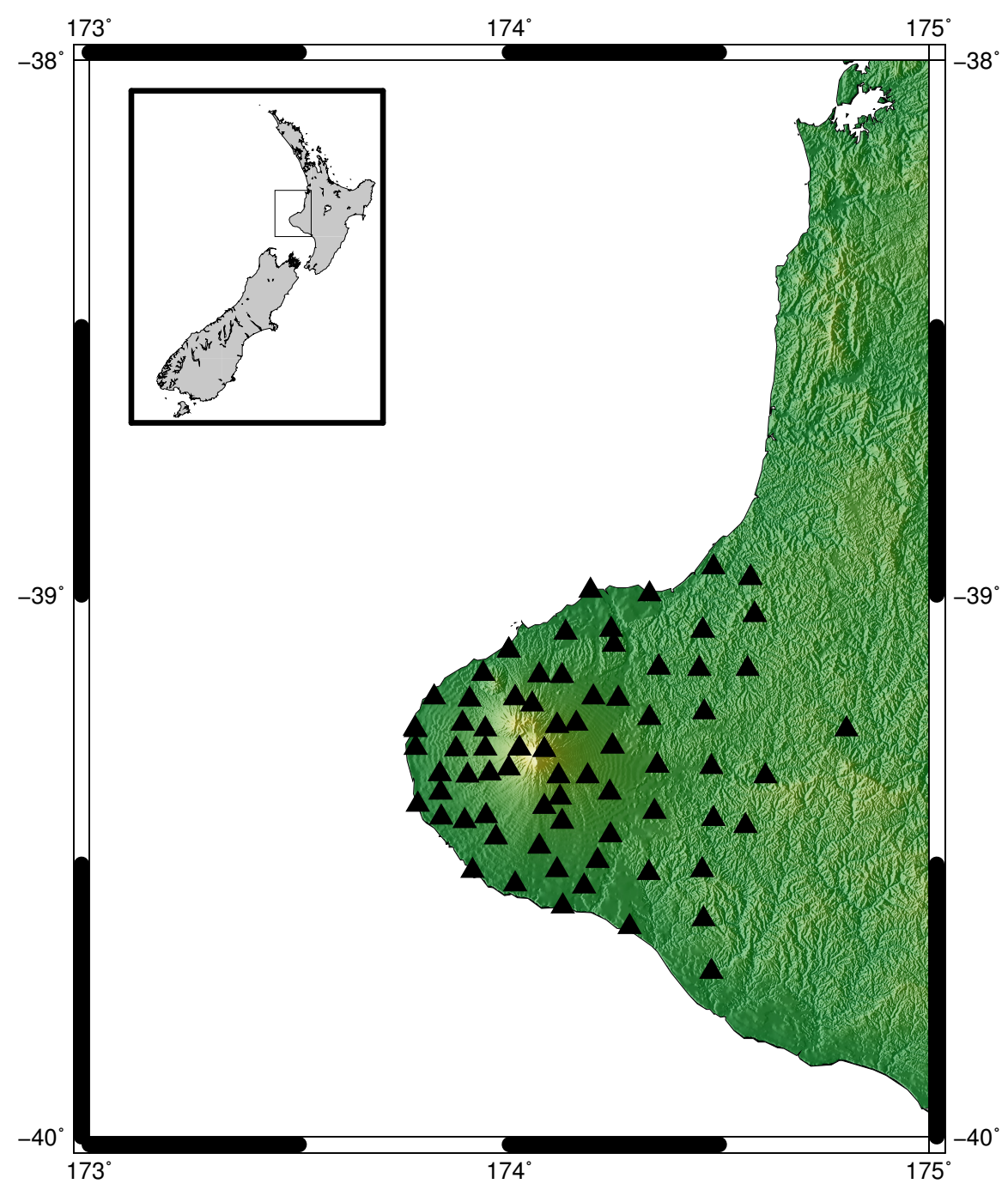

Figure 6.1 Station locations of the Mt. Taranaki deployment marked as black triangles. 


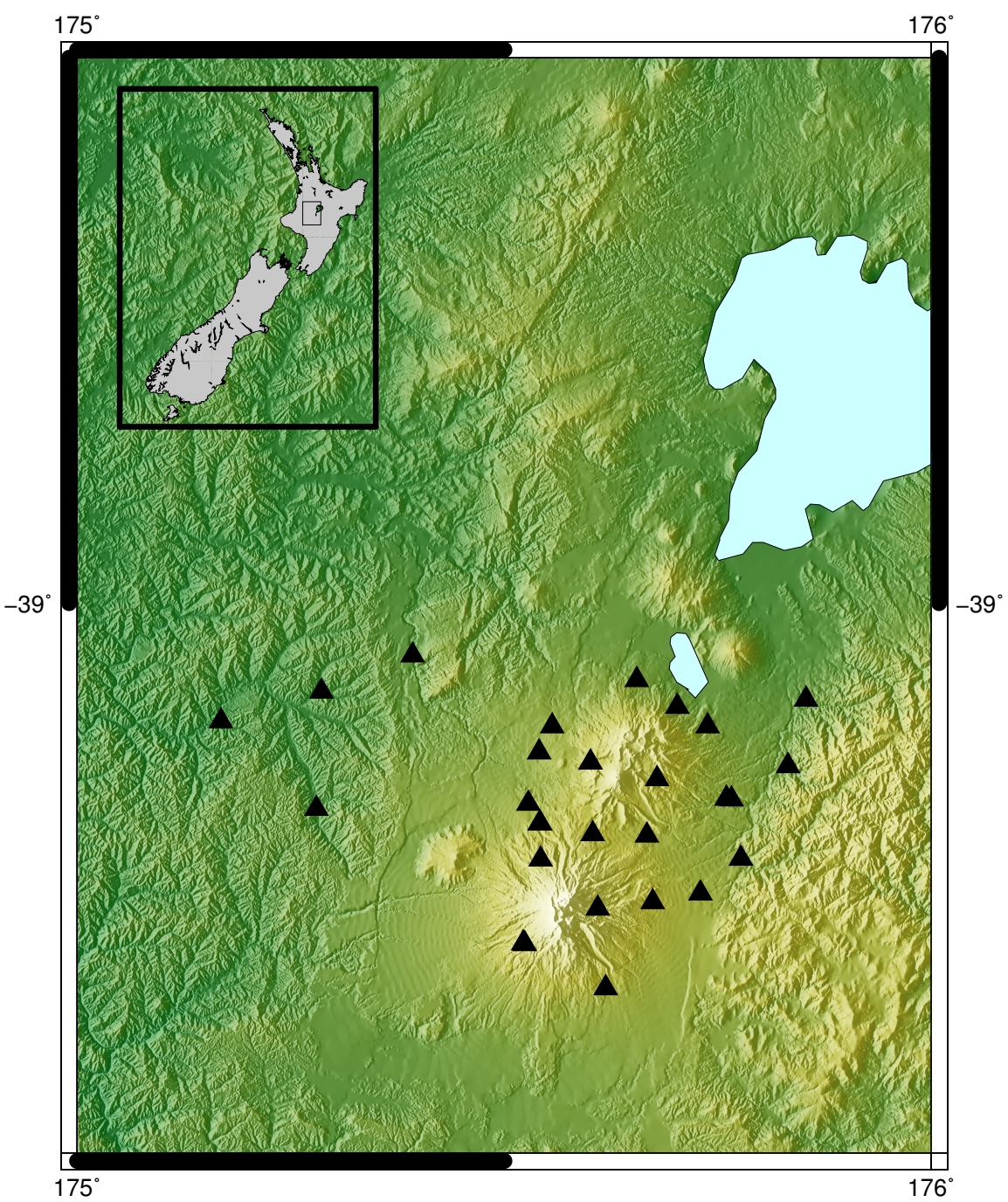

Figure 6.2 Station locations of the START deployment marked as black triangles. 

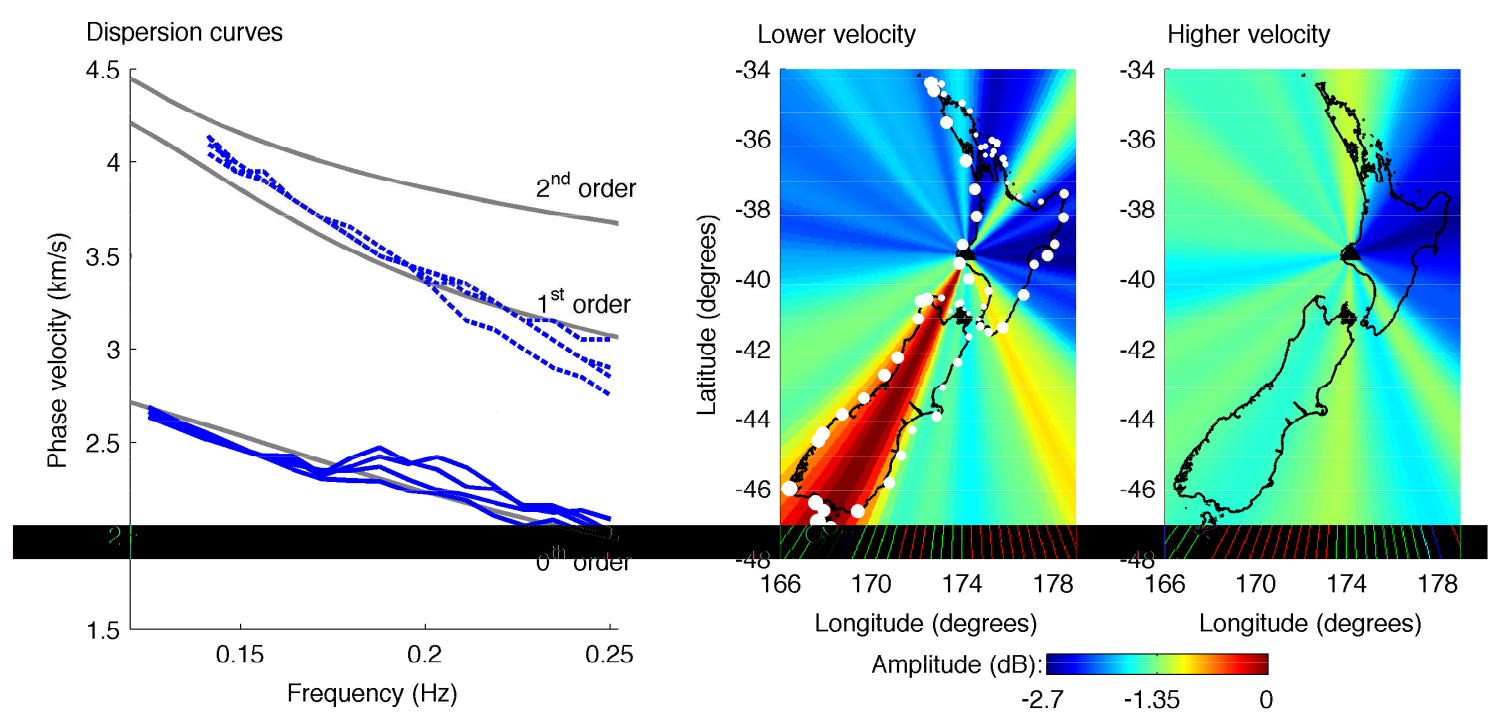

Figure 6.3 Left panel: Dispersive signals from beamformer results of four months (solid and dashed blue lines) on top of theoretical dispersion curves for the fundamental and the first two higher Rayleigh wave modes (solid grey lines). Middle and right panel: backazimuth directions of lower-velocity and highervelocity signal colored according to their beamformer amplitude. Modified after Figure 3 of Brooks et al. [2009a].

signal correlates well with the dispersion curve of the theoretical first higher Rayleigh wave mode but exhibits larger discrepancies with respect to the theoretical curve than the lower-velocity signal does to the theoretical fundamental Rayleigh wave mode. The discrepancies may stem from inaccuracies in the 1D velocity model used to calculate the theoretical dispersion curves or from contamination of the higher-velocity signal by higher modes.

The source directions of the two signals are observed to differ, with the lower-velocity signal (fundamental mode Rayleigh wave) arising mainly from southsouthwest, southeast and northeast directions and the higher-velocity signal (first higher mode Rayleigh wave) being generated across a more continuous azimuthal range extending from southeast $\left(\sim 120^{\circ}\right)$ to north $\left(\sim 0^{\circ}\right)$ azimuths (Figure 6.3 middle and right panels).

\section{Multimode dispersion curves from slant stacking}

Due to the excitation of the primary and secondary microseism on the Earth's surface, higher Rayleigh wave modes are less efficiently excited than fundamental mode Rayleigh waves [Tsai, 2010]. In spite of this, it has been previously demonstrated that higher mode information can also be extracted from the ambient noise field using noise crosscorrelation methods [Harmon et al., 2007; Nishida et al., 2008]. The more continuous 
distribution of source backazimuths observed for the higher-mode signal than for the fundamental mode signal (Figure 6.3) suggests different excitation mechanisms. Therefore the reason why higher mode surface waves are not observed in all ambient noise studies might be a combination of noise energy and characteristics of the study area, such as proximity to the seashore or near-coastal bathymetry.

We computed noise cross-correlation functions between the 68 stations of the Mt. Taranaki deployment for which data could be recovered [Sherburn and White, 2005] resulting in over 2000 cross-correlation functions. Figure 6.4 depicts a subset of the symmetric correlation functions (Section 2.3.2) as a virtual common shot point gather with $\sim 1$ $\mathrm{km}$ pseudo station spacing. Using equation 6.5, we transform the wavefield of all symmetric cross-correlation functions into a slant stack, with the result displayed in Figure 6.5 for velocities varying between 1.5 and $5 \mathrm{~km} / \mathrm{s}$ in $0.1 \mathrm{~km} / \mathrm{s}$ steps and periods between 0.5 and $10 \mathrm{~s}$.

Regions of large slant stack amplitude in the velocity-period domain correlate well with the dispersion curves from plane wave beamforming of Brooks et al. [2009a] averaged over four months (Figure 6.5). Aliasing is evident at periods less than $\sim 4 \mathrm{~s}$ and therefore at longer periods than predicted by the minimum station spacing (see Section 6.1.2 and dashed black line in Figure 6.5): this is most likely due to the irregular spacing between traces, and the amplitudes of the aliased arrivals are mostly lower than those of the surface wave arrivals, as expected, and can therefore be easily distinguished. Although the fundamental mode Rayleigh wave dispersion curve can be traced to lower periods than the beamforming dispersion curve, we cannot conclusively determine the period range over which the slant stack results are reliable. We therefore restrict the following analysis to the $4-8$ s period range of the beamformer results, considered reliable by Brooks et al. [2009a].

We use the dispersion curves from slant stacking between 4 and $8 \mathrm{~s}$ to infer a $1 \mathrm{D}$ shearvelocity model beneath Taranaki, by employing the Neighbourhood Algorithm (Section 2.4.2). The parameterisation of the search range used in the inversion consists of 22 layers of constant thickness with varying S-velocities. The number of layers and the layer thicknesses are identical to the parameterisation used by Sherburn and White [2005], and the S-velocity is allowed to vary by $20 \%$ around the final model of Sherburn and White [2005]. We invert fundamental and first higher Rayleigh wave modes jointly and separately, with the results displayed in Figure 6.6. The sensitivity kernel at $8 \mathrm{~s}$ period, calculated for the model of Sherburn and White [2005] with the program of Takeuchi and Saito [1988], reveals no S-wave sensitivity to velocities at depths greater than $30 \mathrm{~km}$, and we therefore restrict the analysis of the inversion results to the uppermost $30 \mathrm{~km}$. 
The density distribution (colored area in Figure 6.6) of the ensemble of models resulting from the joint inversion of fundamental and first higher mode exhibits $\sim 0.3 \mathrm{~km} / \mathrm{s}(\sim 11 \%)$ slower velocities than the final model of Sherburn and White [2005] (red line in Figure 6.6 ) in the top $2-3 \mathrm{~km}$ and at $\sim 8-20 \mathrm{~km}$ depth. Below $\sim 20 \mathrm{~km}$ most models show $\sim 0.25$ $\mathrm{km} / \mathrm{s}(\sim 6 \%)$ higher velocities than Sherburn and White's [2005] model. The misfit between the fundamental mode dispersion curve from slant stacking and the curves derived from the 1D velocity models inferred by the Neighbourhood Algorithm are generally small. However, for the first higher mode there is a considerable difference between theoretical dispersion curves and the curve from slant stacking for periods longer than $\sim 5 \mathrm{~s}$. This indicates that it was not possible to find models within the specified search range that matched both the fundamental and the first higher mode dispersion curves equally well. The separate inversion of fundamental and first higher mode dispersion curves shows that the fundamental mode dispersion curve obtained by slant stacking is well fit by models similar to that of Sherburn and White [2005] but that fitting the observed higher mode dispersion curve requires models $\sim 0.5 \mathrm{~km} / \mathrm{s}$ faster than Sherburn and White's [2005] model below $\sim 15 \mathrm{~km}$ depth.

The fact that the Neighbourhood Algorithm was unable to find models, within the parameterisation used, that fit both the fundamental and the first higher mode dispersion curve equally well suggests that the differences between the first higher mode dispersion curve from beamforming and the theoretical one based on the model of Sherburn and White [2005] stems from contamination of the first higher mode dispersion curve by other phases [cf. Brooks et al., 2009a].

\subsubsection{START deployment}

Because of its central inland location, the START deployment is well suited to examining the ambient seismic noise field of the North Island. Furthermore, its array response (Figure 5.2) reveals that backazimuth information from plane wave beamforming can be reliably retrieved within the frequency bands of the secondary microseisms. Slant stacking of noise cross-correlation functions computed between all possible station pairs of the START deployment allows us to retrieve a network-averaged dispersion curve and invert this for an 1D S-velocity model extending to $30 \mathrm{~km}$ depth. 


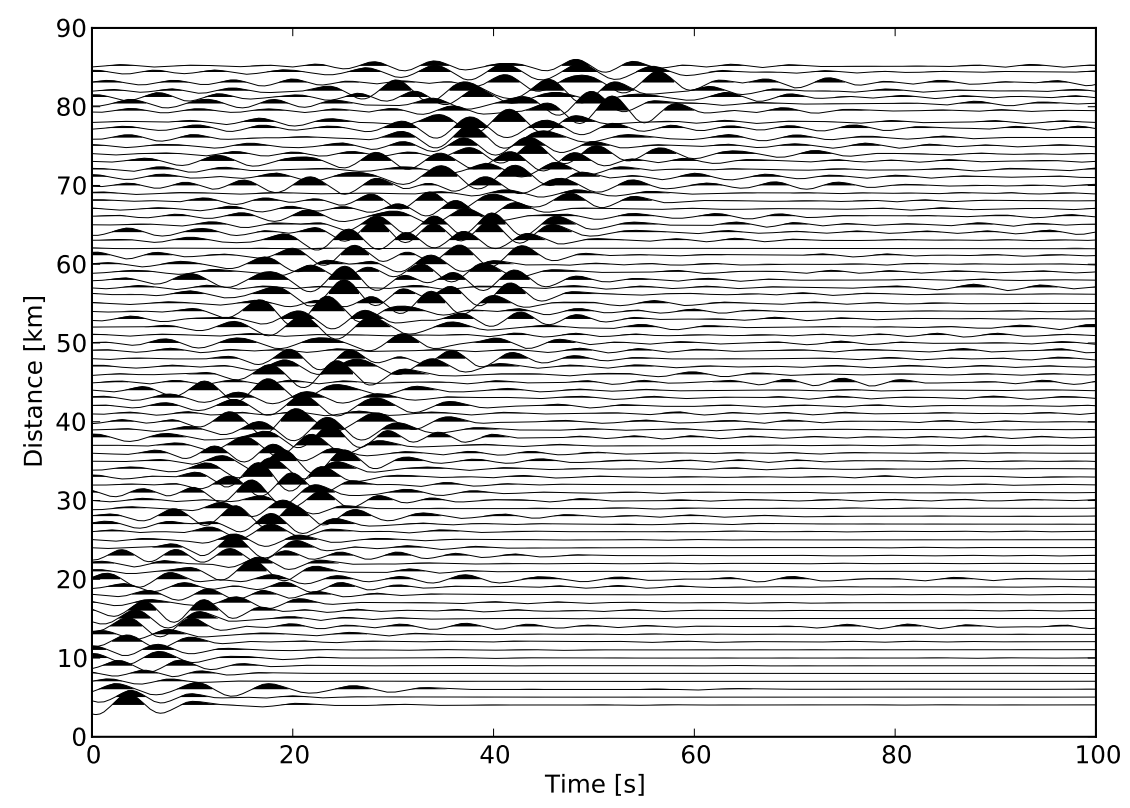

Figure 6.4 Virtual common shot point gather for a subset of the symmetric correlation functions between stations of the Mt. Taranaki deployment with $\sim 1 \mathrm{~km}$ pseudo station spacing.

\section{Seasonal variability from plane wave beamforming results}

As previously mentioned, describing the distribution of ambient seismic noise sources is the first step in establishing the link between ambient seismic noise and the estimated Green's function from noise cross-correlation. Furthermore, if a causal relationship between ocean wave characteristics and the ambient noise field can be found, it may be possible to apply this knowledge to infer physical properties of ocean swells in areas where direct observations are sparse or absent. For example Zhang et al. [2010] could infer the track of Typhoon Ioke in 2006 by employing a spherical beamforming method. Gerstoft et al. [2006a] could relate maxima in plane wave beamforming in California to the time of landfall of hurricane Katrina onto the Mississippi coast in August 2005.

We apply the plane wave beamforming method, described in Section 6.1.1, to 130 days of ambient seismic noise recorded at the stations of the START deployment. We choose days on which ten or more stations were recording simultaneously and focus on the secondary microseism for which the array response (Figure 5.2) shows good resolution. Figure 6.7 shows the beamforming results for 22 February 2001 computed at the 6 s period and for slownesses of 0.2 to $0.5 \mathrm{~s} / \mathrm{m}$ (or $2-10 \mathrm{~km} / \mathrm{s}$ velocity). Also shown in Figure 6.7 is the significant wave height at 68 points along the $50 \mathrm{~m}$ coastal isobath around New Zealand for the same day, taken from the wave prediction model NIWAM [supplied by the Na- 


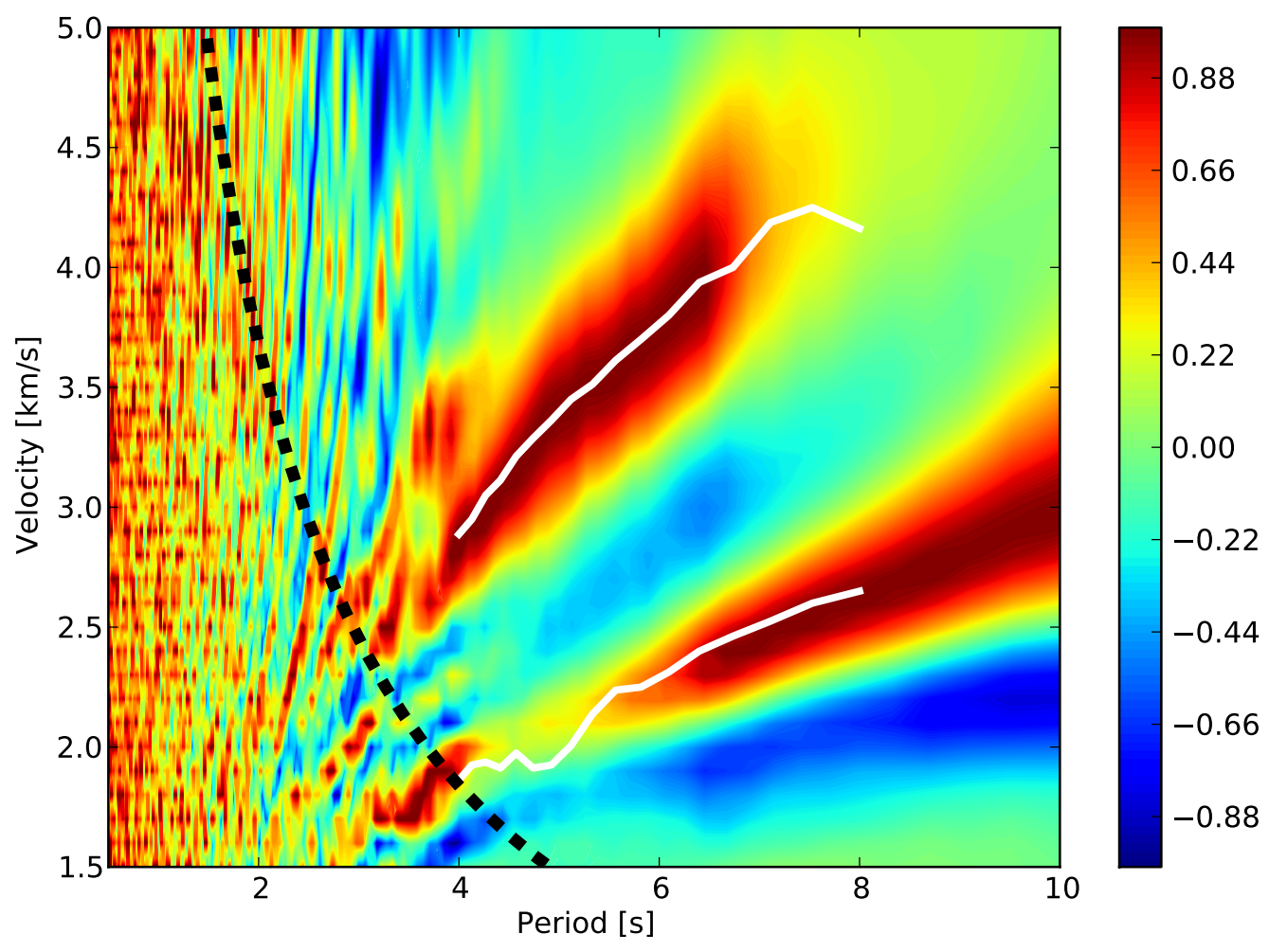

Figure 6.5 Slant stack for the symmetric correlation functions between stations of the Mt. Taranaki deployment. The white lines display the time-averaged dispersion curves obtained from beamformer results by Brooks et al. [2009a]. The dashed black line marks wavelengths of twice times the minimum interstation distance. 


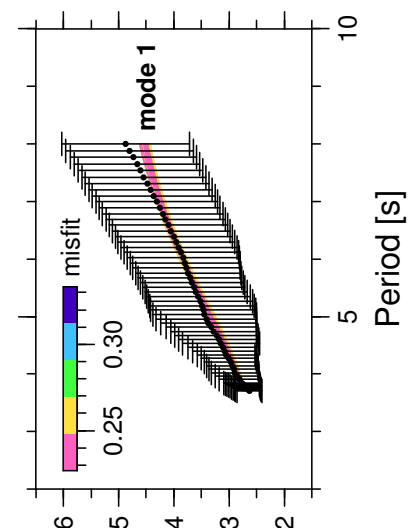

[s/uy] К!

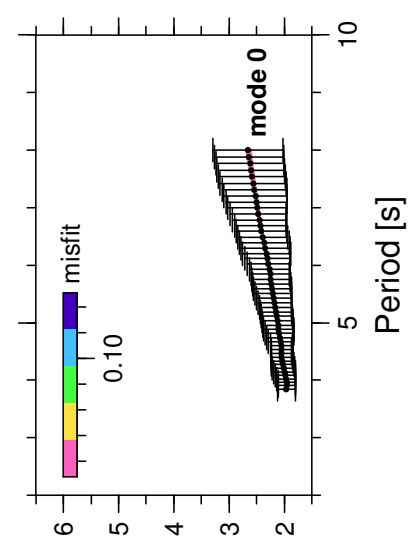

[s/uy] К!잇

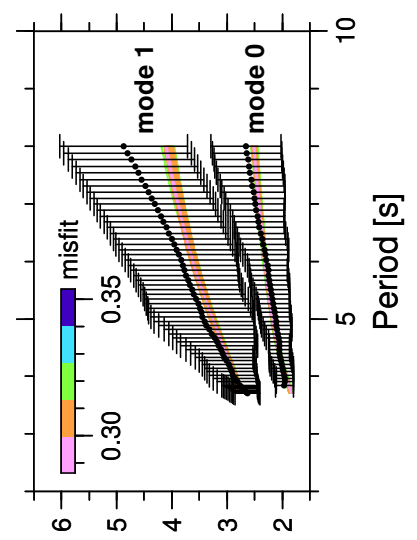

[s/uy] К!잇
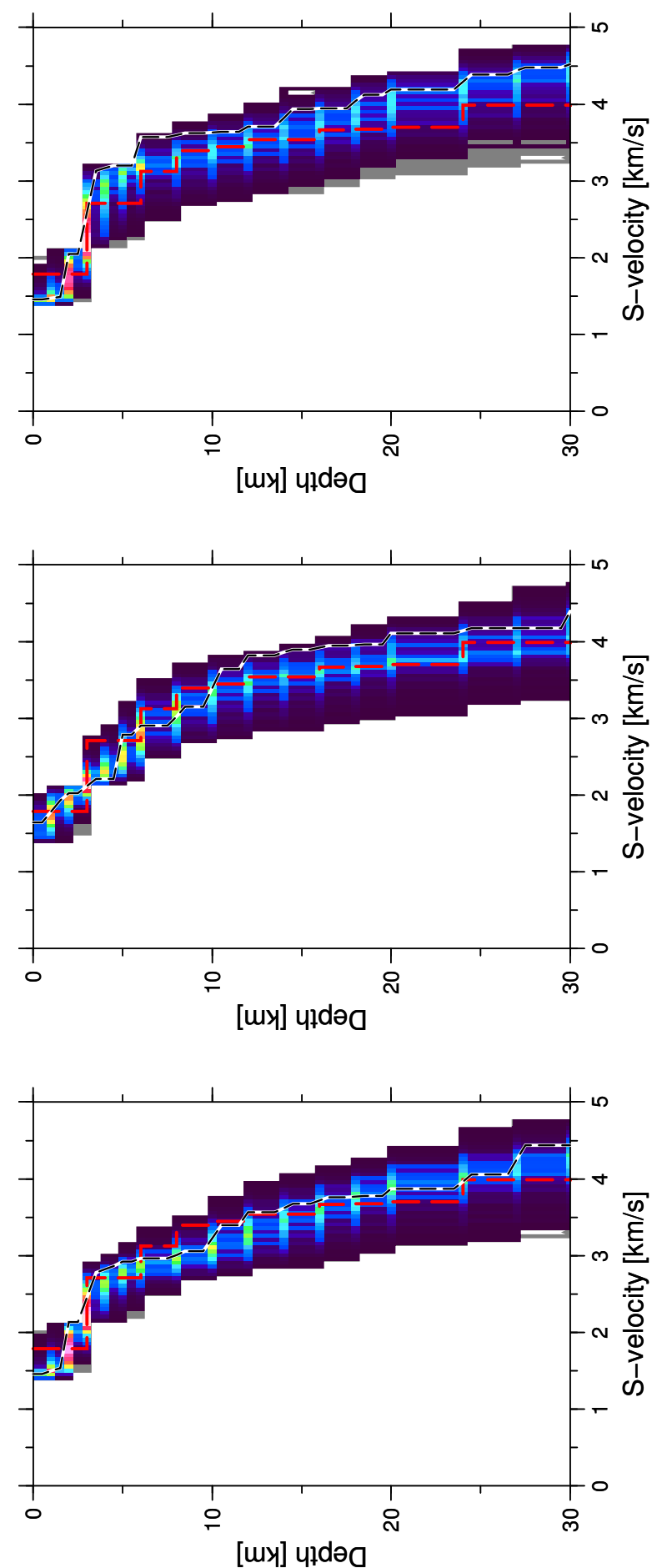

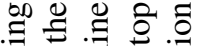

远表焉 क

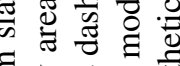

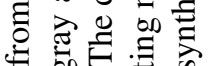
平声 80 ठ․

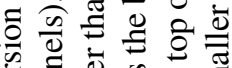

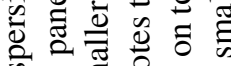

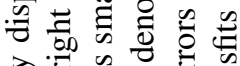

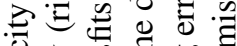
음 \& $\frac{1}{3} \frac{7}{3}$ 定

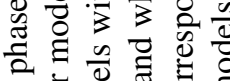
牙

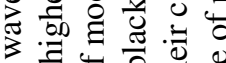
능 0

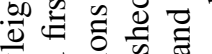

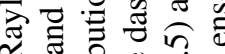
oิ

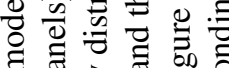

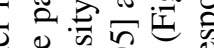

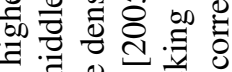
क岁导总导 事 ปี च ฮั

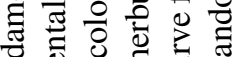
范范 t

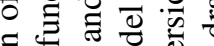

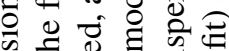

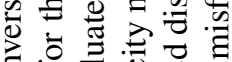

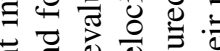

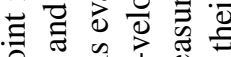

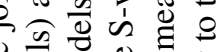
Ð t。

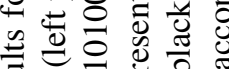

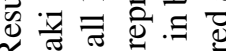
政

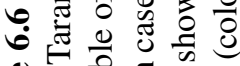

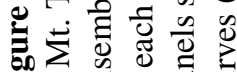


tional Institute of Water and Atmospheric Research, NIWA, http://www.niwa.co.nz, see also Gorman et al., 2003a, b]. After significant wave heights are corrected for geometrical spreading (using a simplistic scaling by the square-root of the distance), a marked correlation between the backazimuth corresponding to the area of maximum amplitude at $0.3 \mathrm{~s} / \mathrm{m}$ slowness in the beamformer result and the azimuthal distribution of ocean wave energy flux and significant wave height is evident. The same comparison for the 3 March 2001 (Figure 6.8) shows weaker correlation between the beamformer results and the significant wave height model, or the ocean wave energy flux. Conversely, a much higher correlation between beamformer output and ocean wave characteristics is apparent in the results for that day at $8 \mathrm{~s}$ period (Figure 6.9). This implies that the differences between significant wave height and the $6 \mathrm{~s}$ beamformer results on 3 March 2001 are due to more energy being transferred into the noise field by ocean waves of $\sim 8 \mathrm{~s}$ period than by those of $\sim 6 \mathrm{~s}$ period. The significant wave height is defined as the average wave height of the one-third largest waves [Brown et al., 1989] and therefore does not convey any information on the period of the waves. This interpretation is reinforced by examining a plot of mean wave period (Figure 6.10) which shows that on 3 March 2001 most energy was transferred from the east coast at periods between $\sim 7$ and $8 \mathrm{~s}$. The results in Figure 6.7 point to strong seismic noise sources in eastern directions at $6 \mathrm{~s}$ period but Figure 6.10 reveals that on 22 February 2001 the mean period on the East Coast of the North Island was approximately $8 \mathrm{~s}$. This shows, that mean period is most likely not accurate enough to fully describe the distribution of ocean wave energy with respect to period.

The beamformer results averaged over all 130 days show good agreement with the significant wave height and ocean wave energy flux averaged over the same time-period (Figure 6.11 ), indicating that daily fluctuations of dominant ocean wave periods within the secondary microseism might average out over longer timespans. As discussed in Section 5.2, at azimuths corresponding to highly attenuating Taupo Volcanic Zone $\left(\sim 355-47^{\circ}\right)$, a gap appears in the averaged beamformer result and the correlation between significant wave height and beamformer amplitude decreases.

\section{Slant stacking}

From the virtual common shot point gather of the cross-correlation functions between all useable station pairs in the START deployment (Figure 6.13) we calculate the slant stack at periods of 0.5-10 s and velocities between 1.5 and $5.5 \mathrm{~km} / \mathrm{s}$ (Figure 6.14). Two dispersive wavetrains are evident, as in the slant stacking results for the Mt. Taranaki deployment. Similarities to the fundamental mode Rayleigh wave phase velocity dispersion 

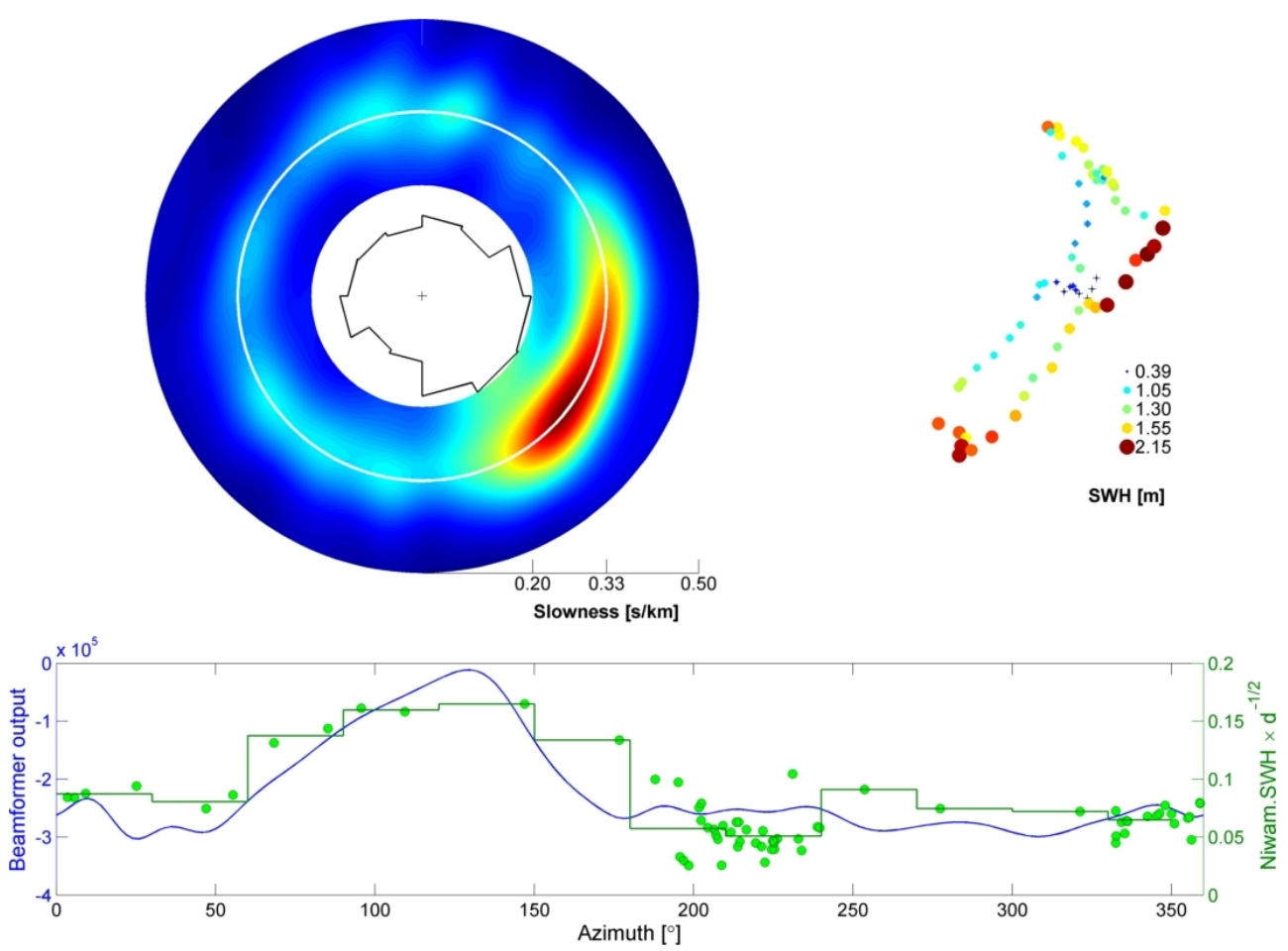

Figure 6.7 Plane wave beamforming results at $6 \mathrm{~s}$ period for ambient noise recorded on 22 February 2001 at 22 seismometers during the START deployment (see Figure 6.2). The upper left panel is the beamforming result with the ocean wave energy flux shown in the middle. The upper right panel shows the significant wave height at the $50 \mathrm{~m}$ isobath taken from the NIWAM model. The lower panel shows the beamformer amplitude as a function of azimuth taken at $0.3 \mathrm{~s} / \mathrm{m}$ slowness as solid blue line and the azimuthal distribution of the significant wave height (green dots) binned at $3^{\circ}$ intervals (green line). To partially compensate for geometrical spreading, each significant wave height datum is divided by the square root of the distance to the center of the START array. 

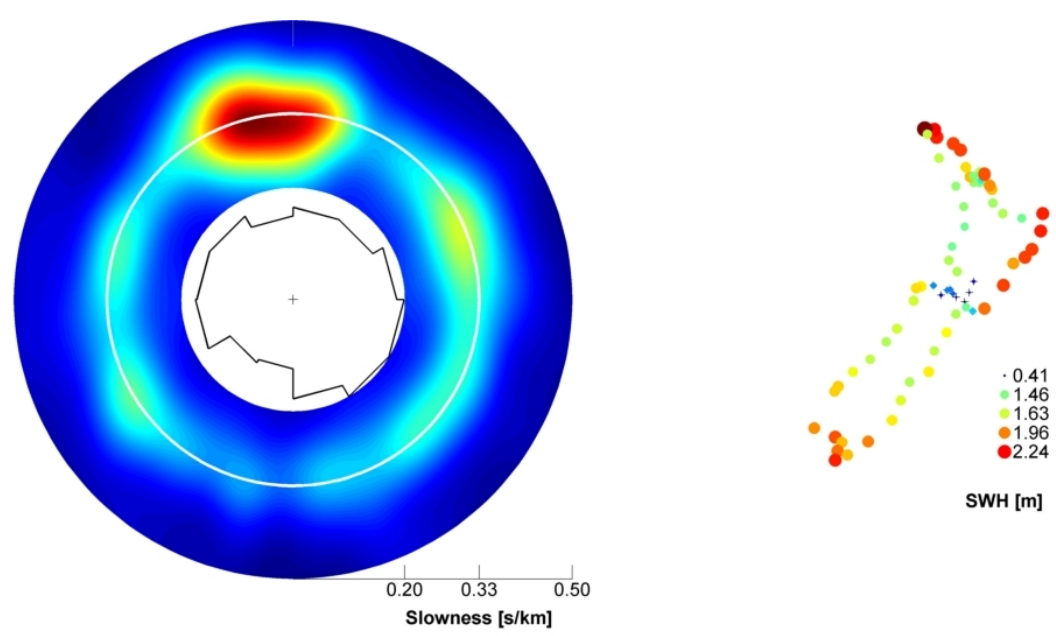

SWH $[\mathrm{m}]$

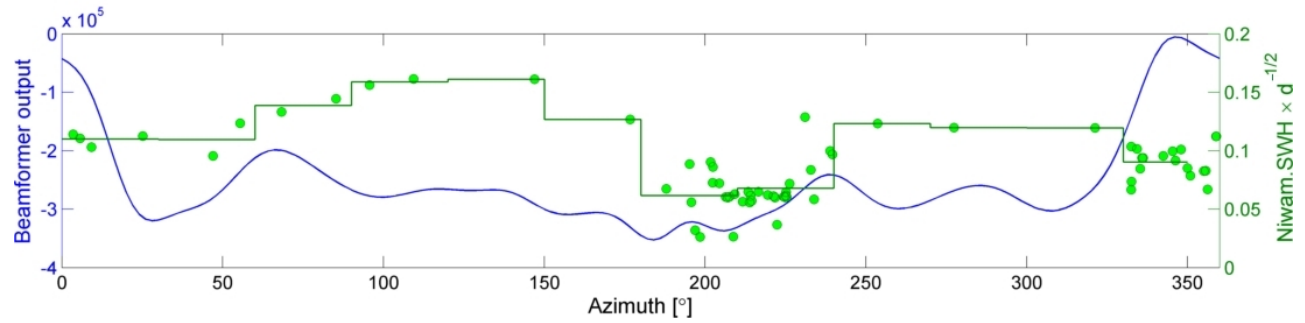

Figure 6.8 Same as Figure 6.7 but for ambient noise recorded on 3 March 2001 at 22 seismometers during the START deployment. Note the large discrepancy between beamformer amplitude and significant wave height between $\sim 60^{\circ}$ and $\sim 180^{\circ}$ and between $\sim 240^{\circ}$ and $\sim 330^{\circ}$.
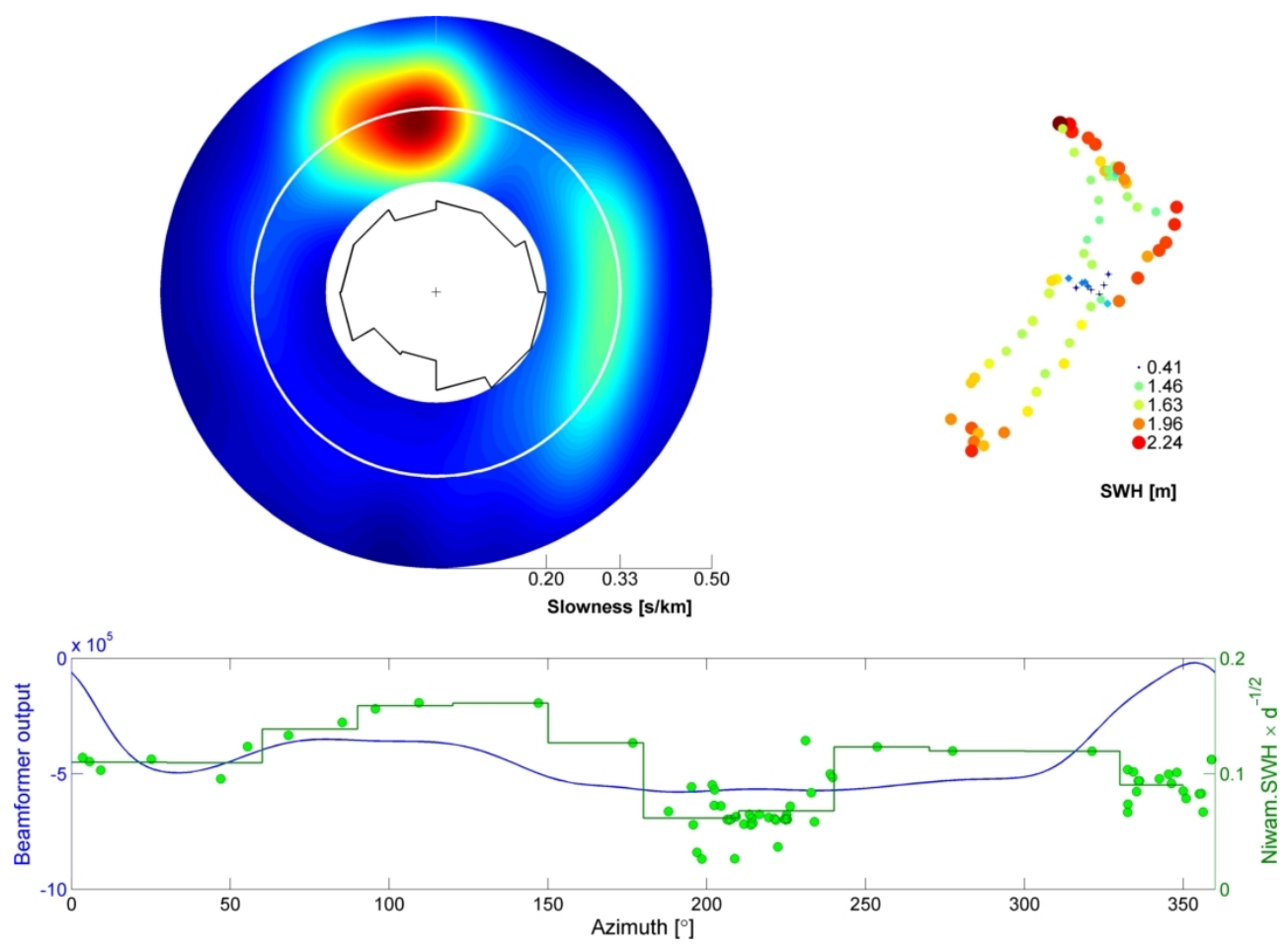

Figure 6.9 Same as Figure 6.8 but for the $8 \mathrm{~s}$ period. The discrepancies between beamformer amplitude and significant wave height, apparent in Figure 6.8, are less evident for the longer-period beamformer output. 
22 February 2001

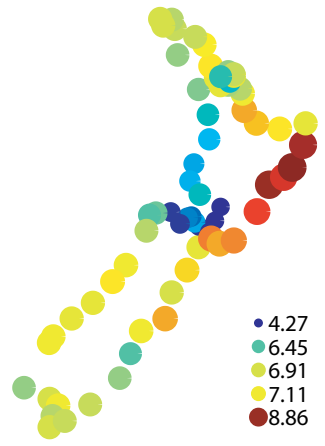

MeanPeriod [s]

\section{March 2001}

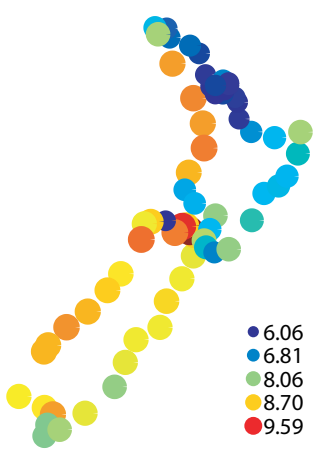

MeanPeriod [s]

Figure 6.10 Mean ocean wave period for 22/2/2001 and 3/3/2001 computed by the NIWAM model.
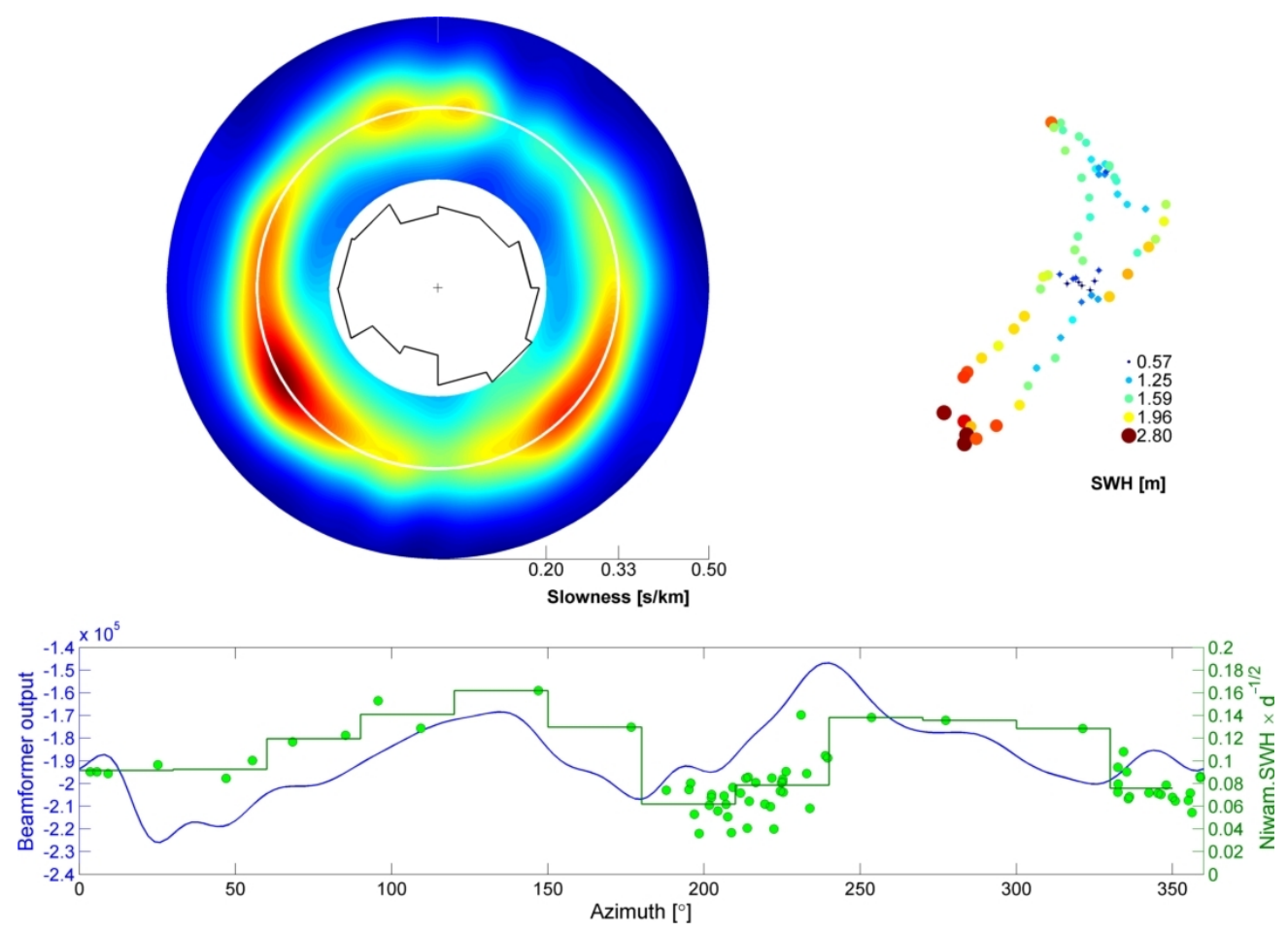

Figure 6.11 Average plane wave beamforming results at $6 \mathrm{~s}$ period for ambient noise recorded by 10 or more stations at 130 days during the START deployment. The upper left panel is the beamforming result with the with the ocean wave energy flux averaged over the same 130 days shown in the middle. The upper right panel shows the significant wave height at the $50 \mathrm{~m}$ isobath taken from the NIWAM model and averaged over the 130 days. The lower panel shows the beamformer amplitude as a function of azimuth taken at 0.3 $\mathrm{s} / \mathrm{m}$ slowness as solid blue line and the azimuthal distribution of the significant wave height (green dots) binned at $3^{\circ}$ intervals (green line). To partially compensate for geometrical spreading each significant wave height datum is divided by the square root of the distance to the center of the START array. In general, azimuthal variations of the beamformer amplitude agree well with those of significant wave height. 
curve inferred at a point within the START array $\left(-39.25^{\circ} / 175.5^{\circ}\right)$ from a suite of surface wave velocity maps at successive periods (Chapter 5) and to the Mt. Taranaki slant stacking results suggest that these coherent features of the slant stack correspond to the fundamental and first higher mode Rayleigh wave phase velocity dispersion curves. To further test this we adopt the lower-velocity signal as the fundamental mode Rayleigh wave and the higher mode signal as the first higher mode Rayleigh wave, in order to invert both for an average S-velocity profile. As aliasing occurs at periods less than $\sim 2.2 \mathrm{~s}$, and the higher velocity signal is only visible at periods less than $\sim 4.5 \mathrm{~s}$, we restrict our analysis of both signals to the period range of $\sim 2.2-4.5 \mathrm{~s}$. We again employ the Neighbourhood Algorithm (Section 2.4) and define the search range around the S-velocity model at $39.2^{\circ} / 175.6^{\circ}$ taken from the global crustal model "crust2.0" [Bassin et al., 2000], with the result shown in Figure 6.15. The search range is restricted to models with S-velocities $40 \%$ faster and $20 \%$ slower and layer thickness differing by not more than $20 \%$ from the a priori model. To add an extra degree of freedom, we split the thickest layer. We use crust 2.0 , because the $1 \mathrm{D}$ S-velocity model corresponding to the point $-39.25^{\circ} / 175.5^{\circ}$ in the pseudo-3D S-velocity model inferred from ambient seismic noise correlations (Chapter 5) appears to be somewhat too slow, especially in the lower crust, to serve as an appropriate prior model (see dashed line in Figure 6.15). It is unclear, whether the pseudo-3D model's velocities are too low or the S-velocity profile deduced from slant-stacking too fast: in Section 5.6 we pointed out that absolute S-velocity values in the pseudo-3D shear velocity model are likely to deviate from the true values, since they are inferred from smooth surface wave maps, which is one possible explanation for the observed difference.

The sensitivity kernel computed for the starting model is zero below $30 \mathrm{~km}$ depth and we therefore constrain our analysis of the inversion results to the upper $30 \mathrm{~km}$ of the crust.

Inverting lower- and higher-velocity signals jointly and then each signal separately yields similar results to the inversion of slant stacking results from the Mt. Taranaki deployment. If the lower- and higher-velocity signals correspond to the Rayleigh wave fundamental and first higher mode, as presumed, the Neighbourhood Algorithm is once again unable to identify models that fit both modes equally well. The density distribution (colored area in Figure 6.15) of the inversion results for the fundamental mode alone matches the prior model (red line in Figure 6.15) at most depths, but the results for the first higher mode by itself tend towards unreasonably high velocities $(>4.3 \mathrm{~km} / \mathrm{s}$ at depths $>8 \mathrm{~km}$ ). 

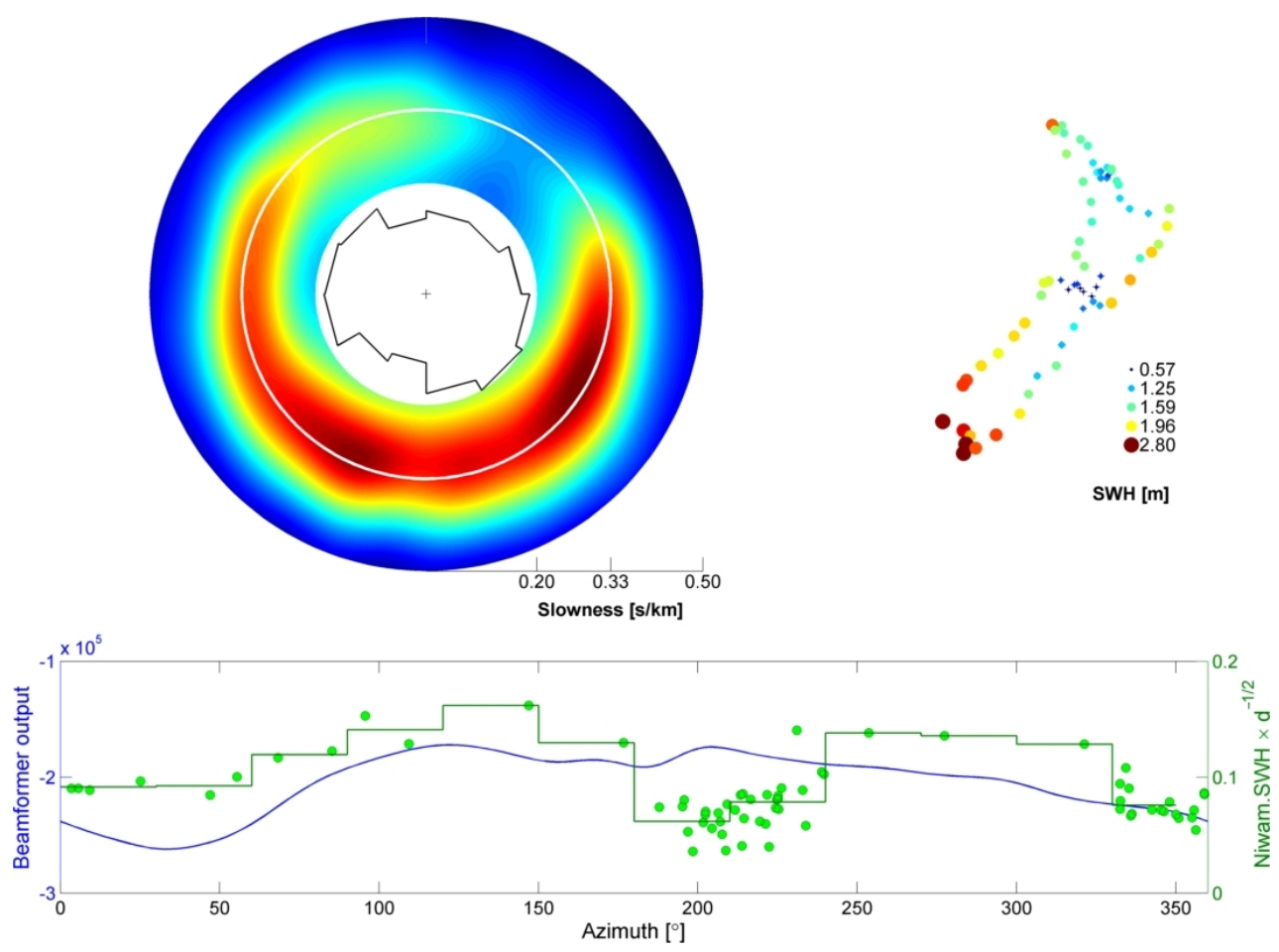

Figure 6.12 Same as Figure 6.11 but for the $8 \mathrm{~s}$ period. Note the difference to Figure 6.11 in the beamformer amplitude for northern and southern azimuths.

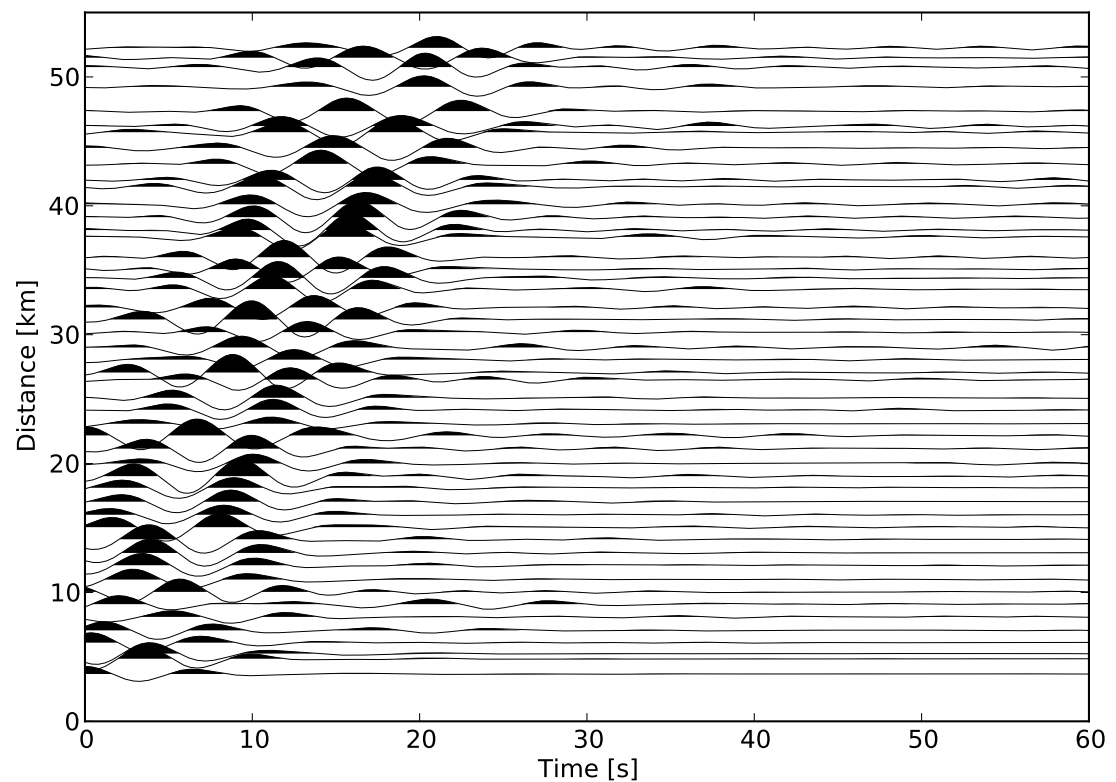

Figure 6.13 Virtual common shot point gather for a subset of the symmetric correlation functions between stations of the START deployment with $\sim 1 \mathrm{~km}$ pseudo station spacing. 


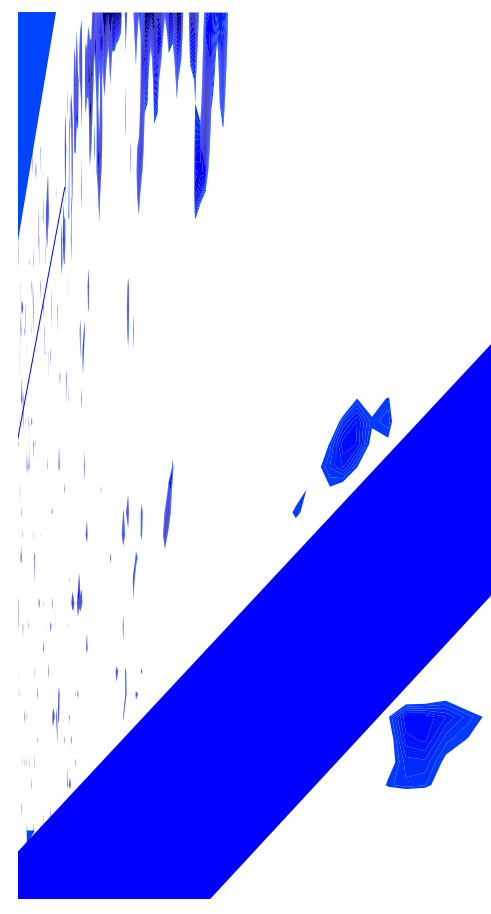

Figure 6.14 Slant stack for the symmetric correlation functions between stations of the START deployment. The dashed black line marks wavelengths of twice the minimum interstation distance. The white line is the fundamental mode Rayleigh wave phase velocity dispersion curve calculated from the pseudo-3D shear velocity model in Chapter 5 at the point $-39.25^{\circ} / 175.5^{\circ}$. 


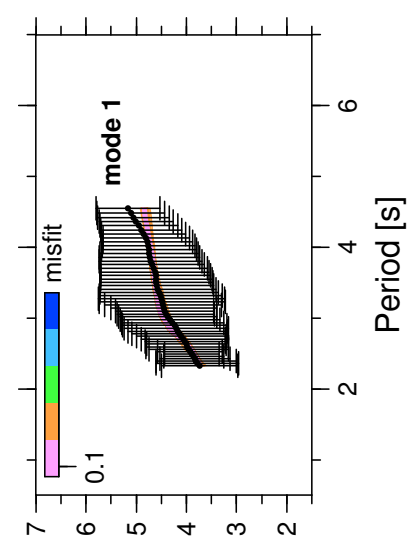

[s/uy] К‡!잇

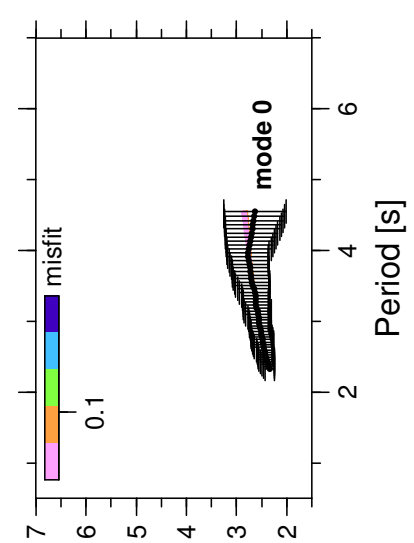

[s/ury] К!잇

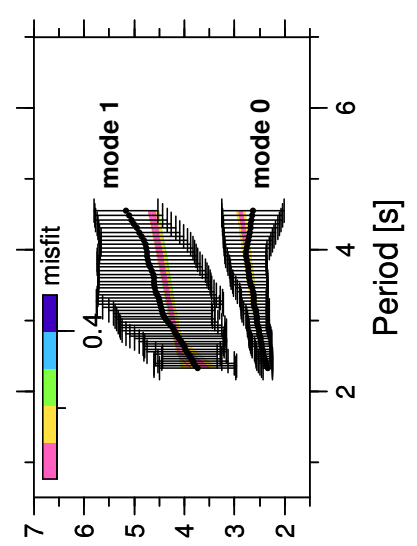

[s/uy] К!ㅟㅇㅣ $\Lambda$
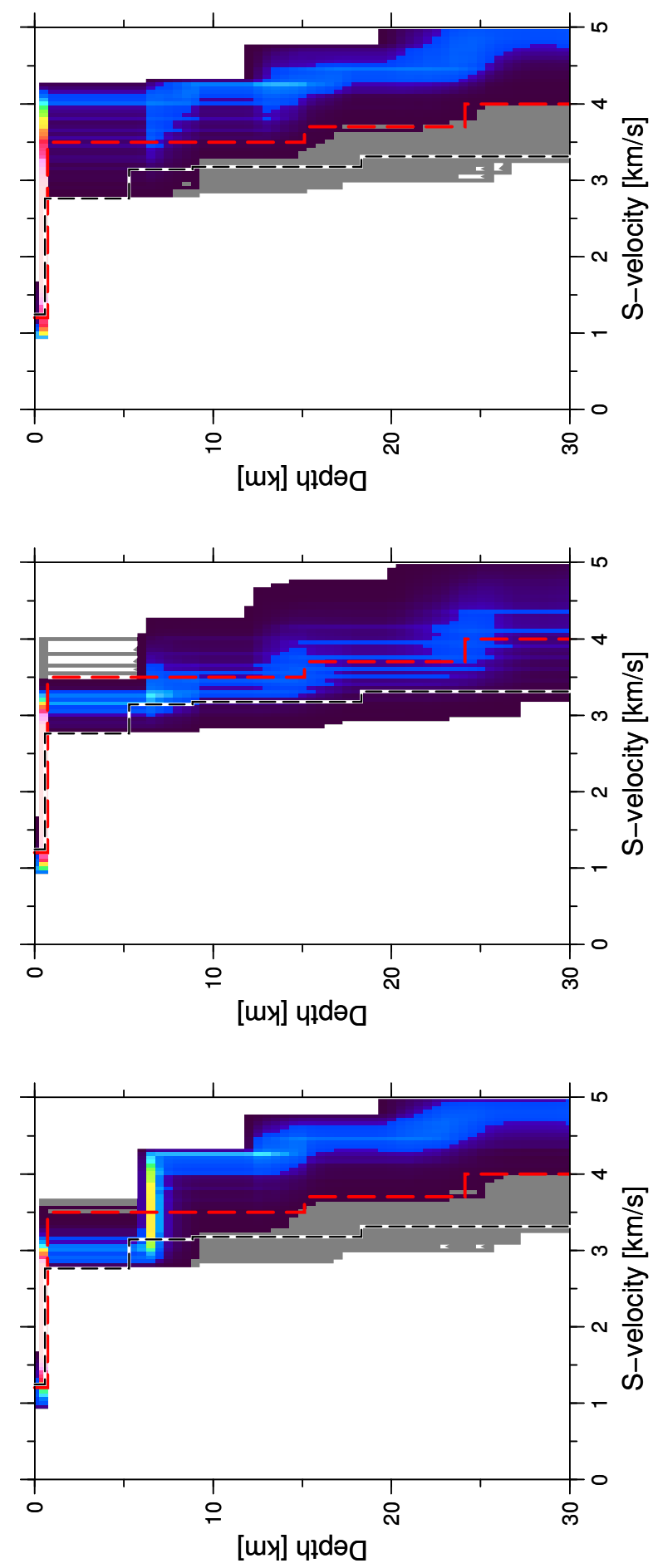

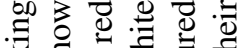

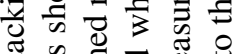

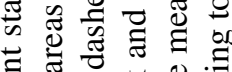
击

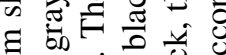
잉

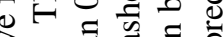

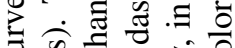

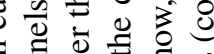

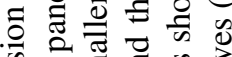

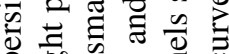

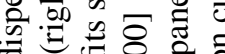

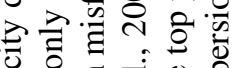

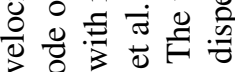
\& $\frac{a}{0}$ in

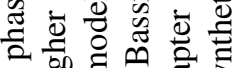

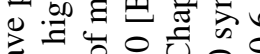

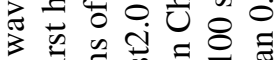

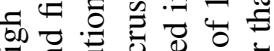
बे

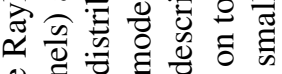

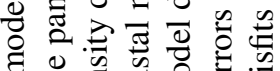

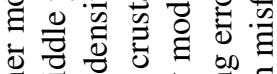

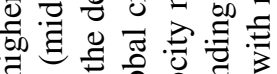
क्षे

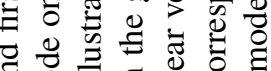

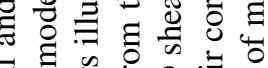

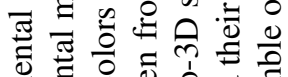

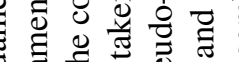

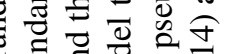

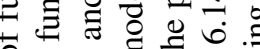

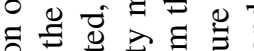

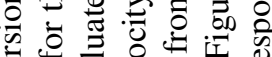

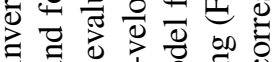

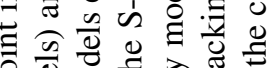

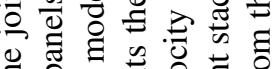
‡

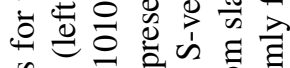

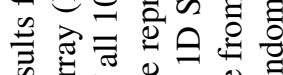

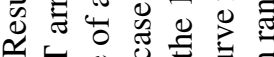

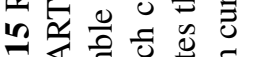

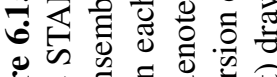

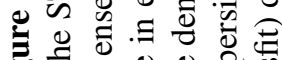

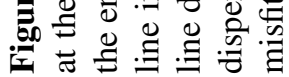




\subsection{Discussion and conclusion}

Gorman et al. [2003a] described New Zealand's ocean wave climate as one of the most energetic on Earth, as a consequence of its being generated by strong winds in the Southern Ocean and occasional ex-tropical cyclones from the north. This results in ocean waves and swell hitting the southwest-, south- and southeast-facing coastlines of the North Island from southwestern or southern directions, and those coastlines facing north and northeast mainly from northeastern directions. From the coasts of the southern South Island to the northern North Island, mean significant wave heights decrease from $\sim 9.8 \mathrm{~m}$ to $\sim 7 \mathrm{~m}$ and mean periods decrease from $\sim 8 \mathrm{~s}$ to $\sim 6 \mathrm{~s}$. The decrease in significant wave height is readily observable in the average beamformer at $6 \mathrm{~s}$ period (Figure 6.11), which shows the highest amplitudes in the southwest and southeast of the START array, with a notable decrease directly to the south. This amplitude low is not discernible in the average beamformer results at $8 \mathrm{~s}$ period (Figure 6.12), reflecting the observation that ocean waves hitting the southern-most North Island have a longer mean period than waves hitting the coastline further northeast and northwest [Pickrill and Mitchell, 1979]. Gorman et al. [2003a] reported mean periods of $\sim 6 \mathrm{~s}$ on the north-facing coastlines of the North Island, which correspond to high amplitudes in the $6 \mathrm{~s}$ beamformer results for waves coming from the north, but relatively low amplitudes for the same directions in the $8 \mathrm{~s}$ beamformer result. As previously mentioned, the highly attenuative TVZ, which lies to the north-northeast of the START array, prevents strong signals arriving from these directions making the START array insensitive to the prevailing ocean conditions in the Bay of Plenty. The fact that our beamformer results correlate well with significant wave heights on the continental shelve is a strong indicator that this is the main source region for seismic noise within the secondary microseism in New Zealand [Kibblewhite and Ewans, 1985]. Our results agree with those of Gerstoft and Tanimoto [2007] for southern California, who inferred source regions of the secondary microseism to lie in shallow waters, on the basis of results obtained using the same plane wave beamforming approach.

Yao and Van Der Hilst [2009] used a 2D plane wave propagation model in combination with $2 \mathrm{D}$ phase velocity maps to model noise cross-correlation functions from noise source distributions in southeast Tibet. To infer and correct for the phase velocity bias arising from an inhomogeneous noise source distribution, they implemented an iterative inversion procedure, which inferred the azimuthal noise source distribution directly from noise cross-correlation results and their corresponding 2D phase velocity maps. From the noise source distribution, inferred from time asymmetries in the noise crosscorrelation functions, theoretical noise cross-correlation functions were calculated which were then used to correct the original cross-correlation functions for phase velocity bias. 
Harmon et al. [2010] extended this approach to 3D plane wave modelling, and used noise cross-correlation functions as well as plane wave beamforming of seismic noise to infer the distribution of noise sources in southern California. They showed, that for noise at periods longer than $7 \mathrm{~s}, 2 \mathrm{D}$ plane wave modelling describes cross-correlations most accurately, but that at shorter periods, 3D plane wave modelling is more appropriate. They further demonstrated that inhomogeneous noise source distributions affect estimates of azimuthal anisotropic phase velocity and attenuation more severely than estimates of isotropic phase velocity.

The good agreement between the dispersion curves obtained by plane wave beamforming and those from slant stacking reflects the conceptual similarities of the two approaches. For example the cross-covariance matrix $\mathbf{C}=\mathbf{W Y} \mathbf{Y}^{\dagger} \mathbf{W}^{\dagger}$ in equation 6.2 is effectively a matrix of noise cross-correlation and auto-correlation functions between each possible station pair in the array. Harmon et al. [2010] therefore used noise cross-correlation functions directly for plane wave beamforming, by setting C's elements on and below the leading diagonal to zero.

The poor fit to the first higher mode dispersion curve obtained when jointly inverting fundamental and first higher modes for S-velocity structure, and the unrealistically fast S-velocity profiles resulting from inverting the higher mode dispersion curve alone, suggest that the higher-velocity dispersion curve is not a pure first higher mode Rayleigh wave dispersion curve. In fact, in the case of the higher velocity dispersion curve measured at the START array, both dispersion curves can be fit satisfactorily if the higher velocity dispersion curve is interpreted as the second higher Rayleigh wave mode rather than the first higher mode (Figure 6.16). This is not true for the higher velocity dispersion curve obtained at Mt. Taranaki, which implies that the observed discrepancies have different causes. Mode coupling between between Love and Rayleigh wave modes due to anisotropic structure as described by Yu and Park [1994] at longer periods $(T>70 s)$ might also play a role at such short periods. Considering the width of the higher velocity signal in the slant stacking results, it is also possible that different modes cannot simply be distinguished by this method. Figure 6.16 shows that both first and second higher mode corresponding to the best fitting S-velocity profiles lie within the error bars of the higher velocity signal. While it might be beneficial to include higher mode information into the inversion for S-velocity structure, the problem of unambiguously identifying higher modes from noise cross-correlation functions still needs to be solved.

In conclusion, we have shown that plane wave beamforming results exhibit high correlation with known ocean wave patterns, making beamforming a reliable method for 

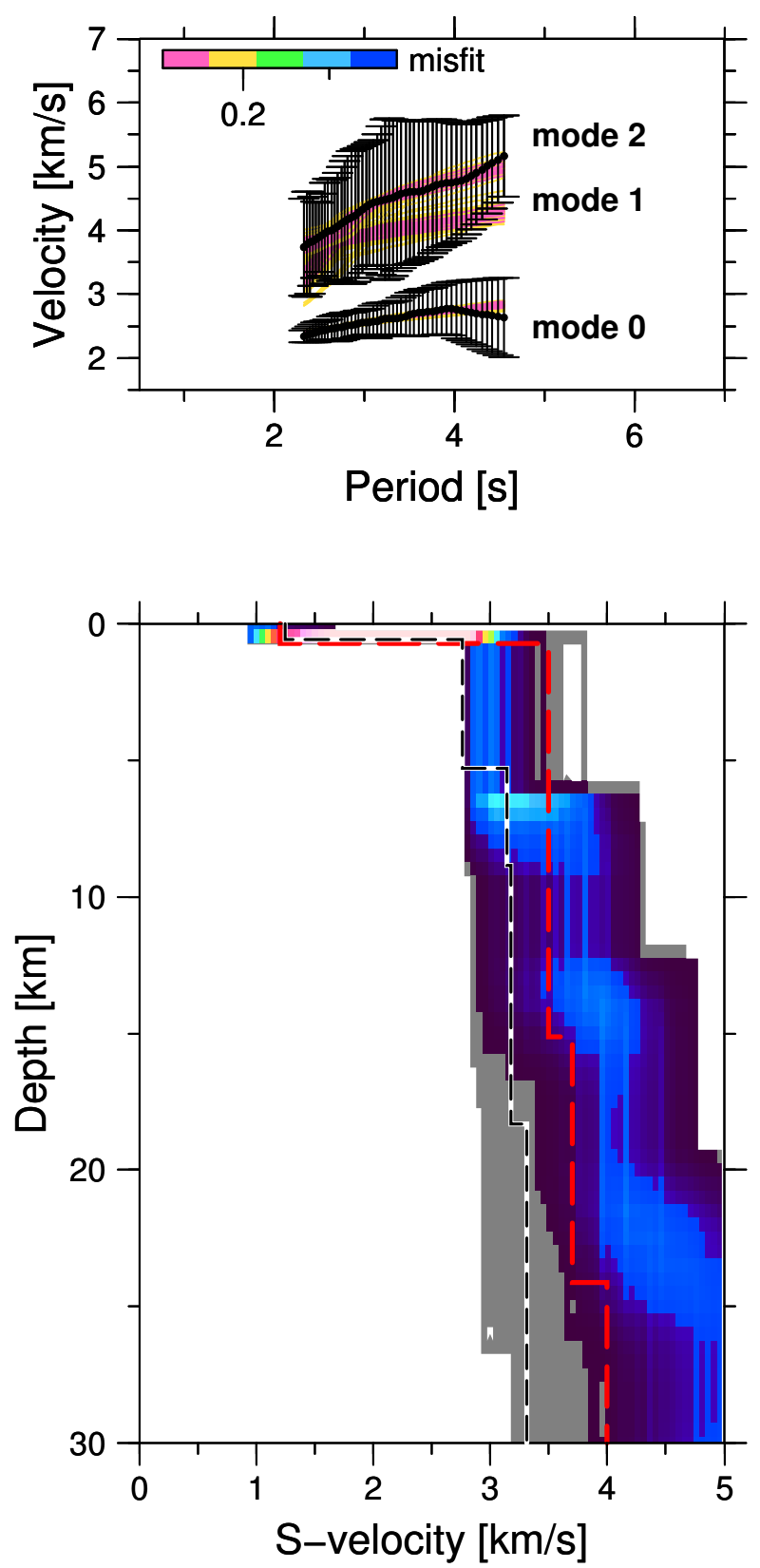

Figure 6.16 Results for the joint inversion of fundamental and second higher mode Rayleigh wave phase velocity dispersion curve from slant stacking at the START array. The gray areas show the ensemble of all 10100 models evaluated, and the colors illustrate the density distributions of models with misfits smaller than 0.6. The dashed red line in each case represents the S-velocity model taken from the global crustal model crust 2.0 [Bassin et al., 2000] and the dashed black and white line denotes the 1D S-velocity model from the pseudo-3D shear velocity model described in Chapter 5. The top panels show the measured dispersion curve and their corresponding errors on top of 100 synthetic dispersion curves that were drawn randomly from the corresponding ensemble of models with misfits smaller than 0.6. For comparison, 100 synthetic dispersion curves corresponding to the first higher Rayleigh wave mode are also shown. 
examining and inferring ocean-generated seismic noise source distributions. Fundamental mode dispersion curves, extracted from beamformer and slant stacking results, can be used to infer a first-order estimate of the S-velocity structure beneath a seismic array and the results are compatible with those obtained by modelling earthquake travel times. Both plane wave beamforming and slant stacking are limited to small- and medium-sized seismic arrays. For large-aperture arrays spanning an area the size of New Zealand, for example, the assumptions of plane waves and lateral homogeneous velocity structure will only be appropriate for very distant sources. If those conditions are not met, spherical beamforming and an accurate velocity model will need to be employed [e.g. Zhang et al., 2010]. Spherical beamforming and a quantitative comparison of beamforming results with data from the NIWAM model are possible avenues of future research. 


\section{Summary of key results and opportunities for further research}

In considering the question of what the analysis of the seismic noise field tells us about crustal structure and about noise source distributions, we have made the following contributions:

Chapter 3:

1. A significantly enlarged dataset of noise cross-correlation functions, which increases the achievable resolution of surface wave maps in the central North and South Island considerably.

2. Extension of the surface wave analysis to horizontal-component seismograms, enabling us to measure both Rayleigh and Love wave velocities.

3. Determination and inversion of phase and group velocities revealing deeper crustal and uppermost mantle seismic structures than possible when analysing group velocities alone.

Chapter 4:

1. Demonstration of the robustness of noise cross-correlation methods in non-ideal recording geometries and regional noise field characteristics by inferring shearvelocity structure from surface wave information recorded on the Northland Peninsula.

2. Inversion of Rayleigh and Love waves for shear-velocity profiles enabling a firstorder estimate of radial anisotropy of $\sim 2 \%$ in the upper crust and up to $15 \%$ in the lower crust. 
Chapter 5:

1. Pseudo-3D crustal shear-velocity models from Rayleigh and Love waves in the central North Island complementing previous seismological studies of compressionalwave structure.

2. Estimates of the presence, distribution and fraction of melts in the crust inferred from pseudo-3D shear velocity models which integrate well with existing volcanological and tectonic models.

Chapter 6:

1. Elucidation of the source regions of seismic noise, inferred from plane wave beamforming, which correlate with near-coastal ocean wave heights in the period band of the secondary microseism.

2. Demonstration that higher-mode surface wave trains can be extracted from the seismic noise field using complementary beamforming and slant stacking methods.

The results obtained in this study highlight how noise cross-correlation studies may be refined in situations in which either the network or the noise field itself are sub-optimal. In order to image structures deeper than the uppermost mantle using ambient seismic noise, the dependence of the maximum observable period on the network geometry poses a serious constraint for a small and isolated region like New Zealand. Moreover, the justification for treating noise cross-correlation functions as estimates of the Green's function is, in practice, largely based on the consistency of results from ambient seismic noise with prior knowledge or results from other geophysical studies. As long as the causal relationship between ambient noise sources and cross-correlation functions is not fully established, these comparisons are the only method of examining the reliability of the seismic noise analysis results.

Consequently, one possible avenue for future research is the forward modelling of crosscorrelation functions using a plane wave approach and the azimuthal noise source distributions inferred from plane wave beamforming. The comparison between modelled and computed noise cross-correlation functions can help reveal the degree of distortion surface waves from noise cross-correlations experience due to inhomogeneous source distributions. As noted previously, studies in southeast Tibet and southern California, adopting this approach, have shown promising results [Yao and Van Der Hilst, 2009; Harmon et al., 2010]. To the best of our knowledge, no study has yet investigated the relation between source distributions and cross-correlation functions for Love waves although it has been shown that the source regions and source mechanisms differ for Love waves to 
those for Rayleigh waves [Friedrich et al., 1998]. Elucidating this causal relationship may clarify why obtaining Love wave dispersion curves from noise cross-correlation functions is in general more problematic than obtaining Rayleigh wave dispersion curves.

Jointly inverting Rayleigh and Love waves for radial anisotropic shear-velocity structure is another promising area for future studies. We show that we are unable to find isotropic shear-velocity models that account for both Rayleigh and Love waves simultaneously, suggesting that radial anisotropic structures are present. Understanding the distribution of radial anisotropy in the crust can help revealing larger geological structures such as dyke or fault alignments and thereby serve as a proxy to understanding local stress and deformation patterns in the crust [Boness and Zoback, 2004]. Taking into account the correlation of features between surface wave maps at successive periods - rather than treating the measurements at each period independently — would be another way to improve the inversion process. One possible approach is the least-squares collocation method [e.g. Seward et al., 2009], which allows to explicitly account for the expected degree of similarities between adjacent surface wave maps. We have begun investigating the possibility of employing least-squares collocation for surface wave tomography beneath Mt. Taranaki.

Monitoring changes in seismic velocities due to tectonic events such as earthquakes or volcanic eruptions is another emerging field in seismology [e.g. Gerst and Savage, 2004; Savage et al., 2010]. Several ambient seismic noise studies have been able to detect such changes after major earthquakes [e.g. Brenguier et al., 2008]. Analysing ambient seismic noise for temporal changes in conjunction with other repeatable seismological experiments such as shear wave splitting may be a further promising research avenue.

Improving methodologies, as previously described, and their application to different parts of New Zealand, such as the central South Island, where station density is particularly favourable for noise cross-correlation analysis, is likely to give further insight into New Zealand's crustal shear-velocity structures. The availability of continuous, high quality, broadband seismic recordings and a geographically dense seismic network plays a crucial role for the analysis of ambient seismic noise. The continuing extension of New Zealand's permanent broadband seismic network is therefore likely to further improve resolution of tomographic images using seismic noise as a source. 
7. Summary of key results and opportunities for further research 


\section{A. Appendix}

\section{A. 1. Data access}

Noise analysis methods require considerably longer timeseries than techniques employing earthquake data. For several reasons, email-based data request tools like the Automatic Data Request Manager, AutoDRM [Kradolfer, 1993, 1996], which are a common way of obtaining earthquake data from data centers, are suitable to only a limited extent when requesting the continuous data needed for ambient noise analysis. First, automating the request and data download are cumbersome and error-prone as both steps involve a lot of text processing and because the email service providers represent an extra stage on the data request workflow which can introduce unpredictable time delays. Second, due to the configuration of email request tools for requesting earthquake data, data requests can be hampered by internal restrictions on the service, such as maximum allowable request sizes and upload bandwidths.

In New Zealand, geophysical datasets are collected, archived and disseminated by GeoNet $^{1}$, a project funded by the New Zealand Earthquake Comission. It is managed and operated by GNS Science, one of the crown institutes in New Zealand. A high-speed Internet connection is provided by the Kiwi Advanced Research Network, KAREN ${ }^{2}$, which connects universities and crown institutes in New Zealand. In collaboration with Paul Grimwood from GNS Science we developed a web service which provides a faster and easier way to access very long continuous timeseries than the existing AutoDRM service (Figure A.1).

The web service is based on the Simple Object Access Protocol ${ }^{3}$, which uses the Web Services Description Language ${ }^{4}$ to define the interface provided by the web service. To build a client application that can communicate with the web service, the interface definition is

\footnotetext{
${ }^{1}$ GeoNet: http://www.geonet.org.nz

${ }^{2}$ KAREN: http://www.karen.net.nz/

${ }^{3}$ SOAP: http://www.w3.org/TR/soap/

${ }^{4}$ WSDL: http://www.w3.org/TR/wsdl
} 
Stage 1

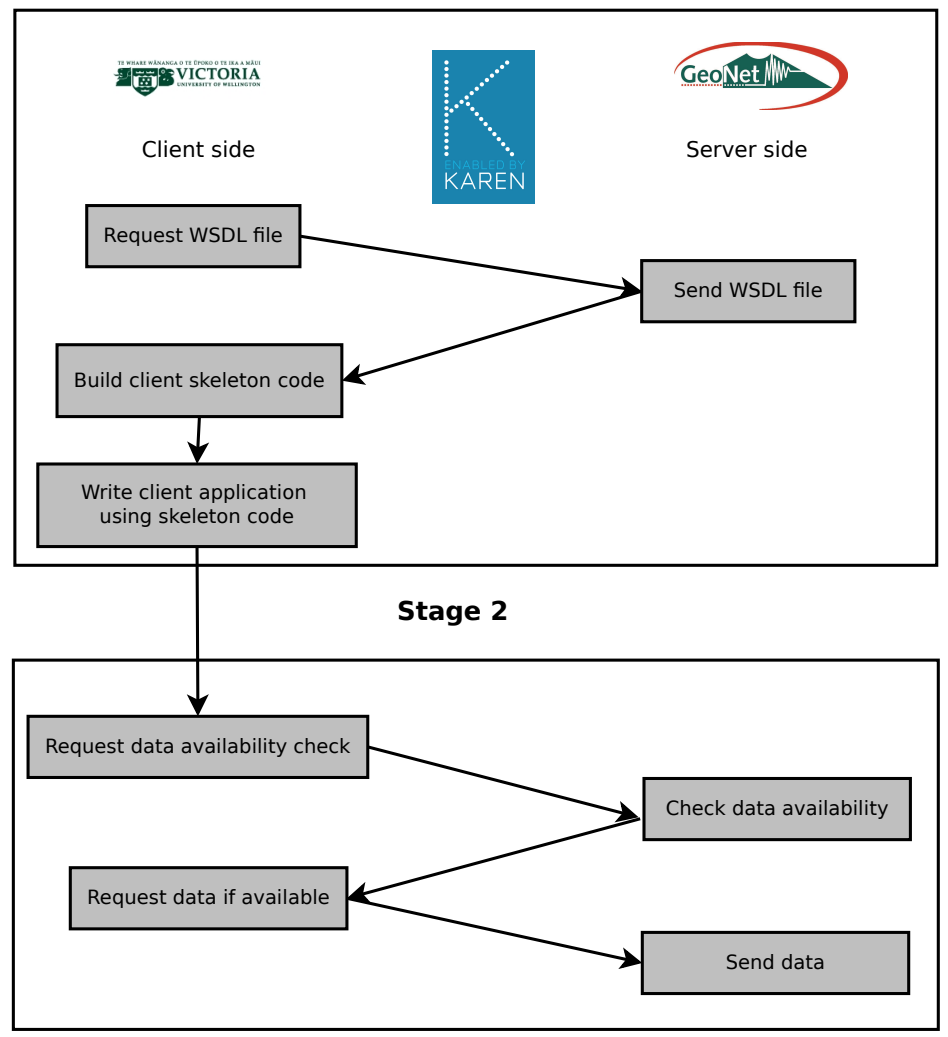

Figure A.1 Schematic representation of the workflow by which continuous waveform data stored by GeoNet can be requested using a web service application via the Karen network. Stage 1 only has to be completed once. After that the client application can be used and Stage 2 is run for each day, station and channel of the request. 
requested from the client side and then used to build source code which provides the basic functionality to, in this case, request data from the web service. The client side code can be build in any programming language which has SOAP support. Simple tools exist to automatically generate the basic client code from the WSDL file, such as gSoap ${ }^{5}$ for the programming languages $\mathrm{C}$ and $\mathrm{C}++$ and the Netbeans IDE for $\mathrm{Java}^{6}$. More user-friendly data request tools can then be written using the source code skeleton that was constructed from the interface specifications. Figure A.1 shows a graphical representation of this workflow. We have successfully implemented client codes in the programming languages Java and C.

The advantage SOAP over alternative methods of interprocess communication, such as, for example CORBA ${ }^{7}$ is, that SOAP uses the Hypertext Transfer Protocol ${ }^{8}$ and standard port 80 for communication and therefore does not require extra ports to be opened on a firewall to permit interprocess communication. At the same time, this advantage also poses a potential security risk and decreases the speed of the communication. However, data access speed is still well above what is obtainable with AutoDRM and we consider the versatility of the web service a more important feature than high-end performance.

Setting up this web service enabled us to include the data request directly into our signal processing workflow and to take advantage of the large bandwidth provided by the KAREN network. However, since we developed this tool for accessing GeoNet data, the Common Waveform Buffer ${ }^{9}$ has been implemented by GeoNet to perform the same but also more complex tasks related to data access. That service only recently became available and fully functional and we therefore continued to use the web service depicted in Figure A.1 throughout this study.

\section{A.2. Grid computing ${ }^{10}$}

Figure A.2 describes the processing flow to calculate ambient noise cross-correlations between the vertical component seismograms recorded at three stations for the same day. The processing steps one to three are obviously decoupled and can therefore be easily parallelized. The necessity to parallelize the processing depends, of course, on the size

\footnotetext{
${ }^{5}$ gSoap: http://gsoap2.sourceforge.net

${ }^{6}$ Netbeans: http://netbeans.org

${ }^{7}$ CORBA: http://www.omg.org/technology/documents/corba_spec_catalog.htm

${ }^{8}$ HTTP: http://www.w3.org/Protocols

${ }^{9}$ CWB: http://www.geonet.org.nz/resources/basic-data/waveform-data/index.html

${ }^{10}$ Grimwood [2008], Grimwood et al. [2008], Behr et al. [2008], Townend et al. [2009a], Townend et al. [2009b]
} 
of the dataset and the sampling interval of the seismograms. Figure A.3 illustrates the runtime depending on the number of stations in the dataset and the sampling frequency. While the runtime increases linearly with the sampling frequency, it increases quadratic with the number of stations as the number of cross-correlations for $n$ stations is $n \times(n-$ $1) / 2$. Not shown here is the linear dependence of the runtime on the number of days in our dataset. This shows that for a typical dataset in ambient noise tomography of, for example, several months of recordings at tens of stations at a sampling frequency of $1-100 \mathrm{~Hz}$, parallelization of the processing routines can result in dramatically reduced runtimes. Moreover, as the nature of the processing lends itself to easy parallelization, only little effort in terms of implementation is required. While the cross-correlation step itself can be easily parallelized, too, it requires the pre-processing to be synchronized, that is finished for the two seismograms that are to be correlated. The work-flow is therefore divided into two stages: the pre-processing and the cross-correlation.

To run the calculation of ambient noise cross-correlations in parallel, we used a grid of 230 desktop computers in the School of Engineering and Computer Science with netBSD as the operating system and SUN Grid Engine (SGE) as the middleware. At the time, the grid was configured as a cycle-stealing grid which meant that each desktop computer was only available as a grid node while otherwise idle. As configured at the time, if a desktop computer was required by its desktop user, jobs running on that node were aborted. This required the implementation of a quality control cycle during which failed jobs were identified and restarted. As this makes scaling of the processing difficult, we ran the processing for 10 stations and 360 days of ambient noise recorded with $100 \mathrm{~Hz}$ sampling frequency at $2 \mathrm{am}$ in the morning, when most desktop computers were idle. The calculations were completed on 180 nodes within three hours, approximately 40 times faster than if the processing had been done on a single-processor desktop computer. The reason why it was not 180 times faster lies in the differences in speed of the grid-nodes and in the computational overhead required to distribute the computation. Grid computing is now commonly used by graduate students in geophysics to run computationally intensive programs. Furthermore, a new grid of 32 nodes is now available within the School of Geography, Environment and Earth Sciences. It is configured as a dedicated grid and quality control cycles, as described above, are therefore superfluous.

\section{A.3. ObsPy}

Conversion of seismological data between different data formats, correcting for instrument response, downsampling and filtering are common components of many processing 


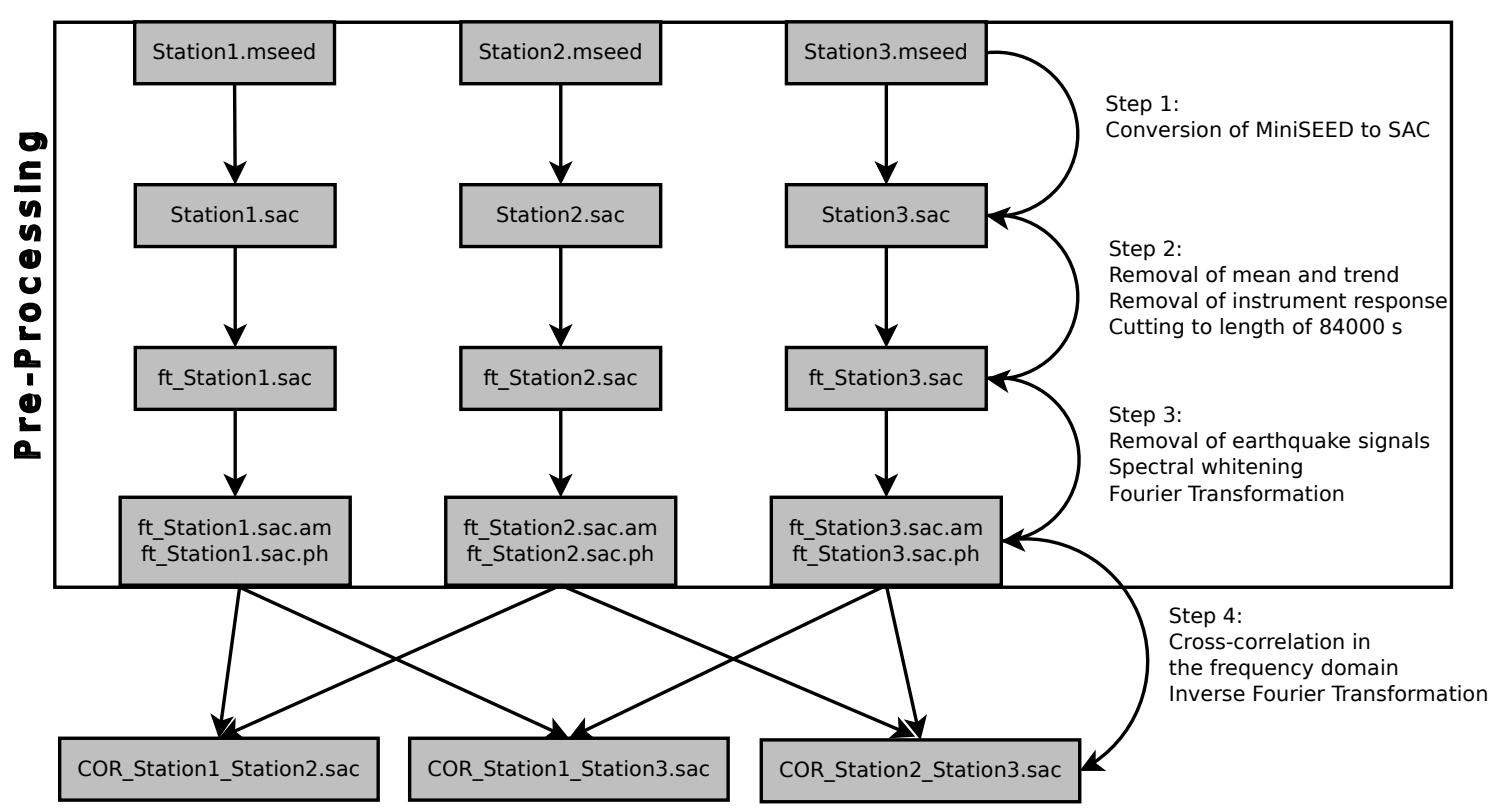

Figure A.2 Processing work-flow diagram to calculate cross-correlation functions from one day of ambient seismic noise recordings at three stations. Details of the processing are described in Chapter 2.3 after Bensen et al. [2007].

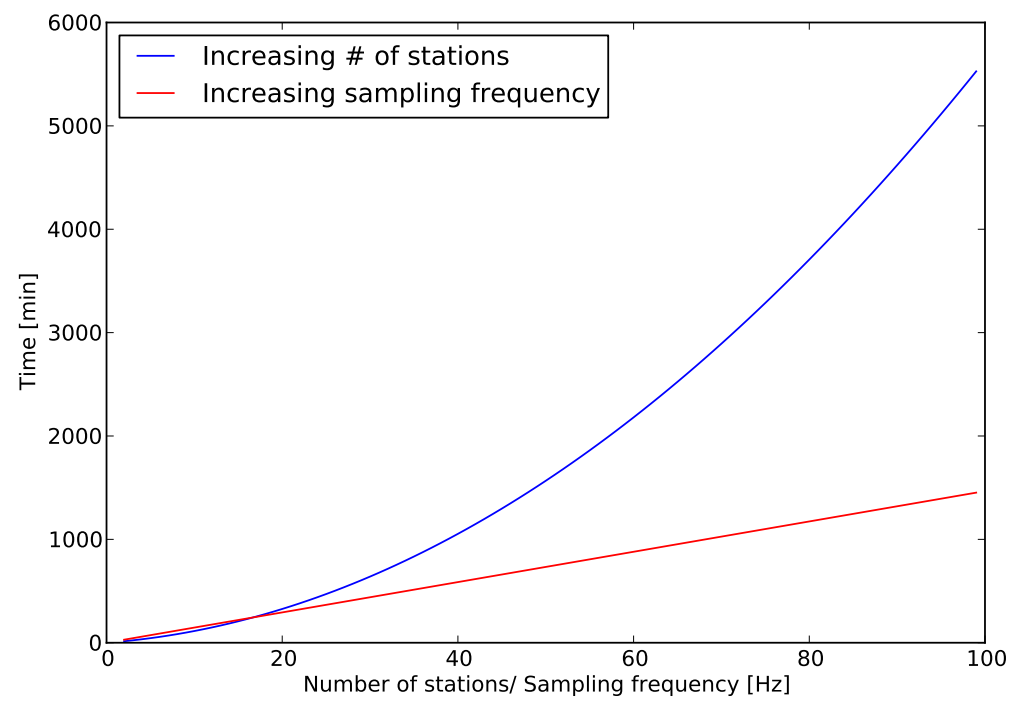

Figure A.3 Dependence of the processing runtime on the number of stations and the sampling frequency. The dependence on the frequency at a constant number of stations is linear whereas the dependence on the number of stations at constant sampling frequency is quadratic. 
work-flows in seismology. However only few software standards exist to date to accomplish these tasks. Projects such as, for example, the Seismic Analysis Software ${ }^{11}$, the Seismic Analysis System ${ }^{12}$ and the CORAL library [Creager, 1997] focus on high-level analysis tasks. ObsPy [see http://www.obspy.org and Beyreuther et al., 2010] is a library based on the open-source interpreter language Python ${ }^{13}$ which has been developed by a team of postgraduate and graduate students (including YB) who are trying to combine their technical knowledge on different data formats and seismological methods to establish a library of software routines, which focus on these most common and most basic tasks in seismological data analysis.

As Python comes with its own package management system pypi ${ }^{14}$ with currently more than 11000 packages and over 700 scientific toolboxes it enables us to focus on the implementation of seismology specific routines without the need to implement, for example, standard signal-processing methods. Data formats currently supported by ObsPy are GSE2,DatalessSEED, MiniSEED, binary and alphanumeric SAC and SEISAN. It provides a unified access method to read seismograms without the need to declare the data format. The following text shows a code-snippet that demonstrates how to read a MiniSEED file, correct for the instrument response, downsample including a lowpass filter, apply a bandpass filter and then write the resulting trace as a SAC-file. The raw seismogram for a local earthquake recorded at the station CRLZ (Canterbury Ring Laser) and the same trace after pre-processing are shown in Figure A.4.

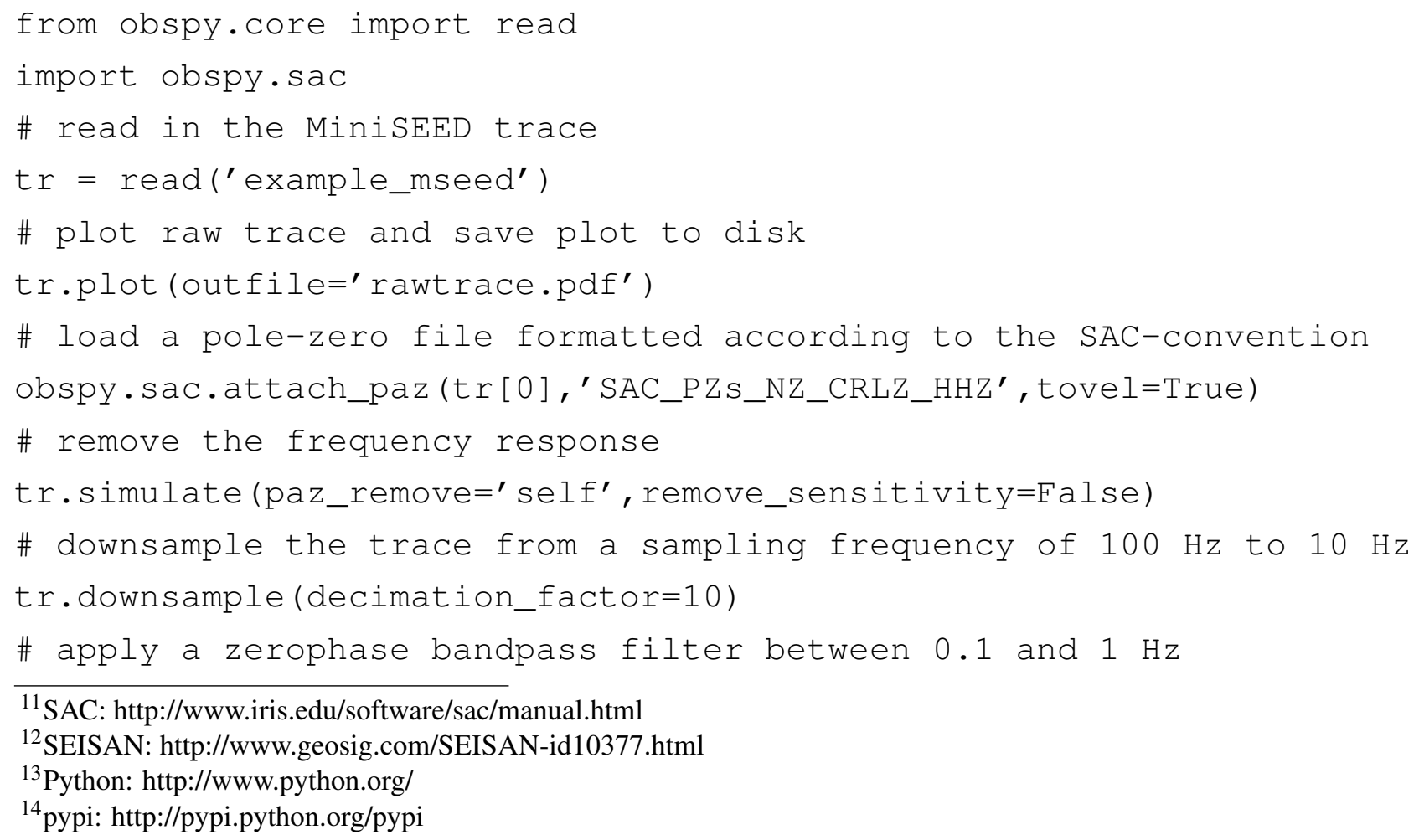


tr.filter ("bandpass", freqmin=0.1, freqmax=1.0, zerophase=True)

\# plot the processed trace and write the plot to disk

tr.plot (outfile='processed_trace.pdf')

\# write result to disk in the SAC-format

tr[0].write ('example.sac', format='SAC')

Apart from these most basic functionalities, several other signal processing methods and interfaces to some common webservices such as the Data Handling Interface (DHI) for the IRIS datacenter ${ }^{15}$ are provided. The following code snippet shows how to request from IRIS the vertical component seismogram at $1 \mathrm{~Hz}$ sampling frequency recorded at the Global Seismograph Network (GSN) station SNZO (South Karori) for the magnitude 7.1 Darfield earthquake that occured west of Christchurch, New Zealand, at 04:36 NZST (16:36 UTC) on 3 September 2010. A plot of the one-hour long seismogram is shown in Figure A.5.

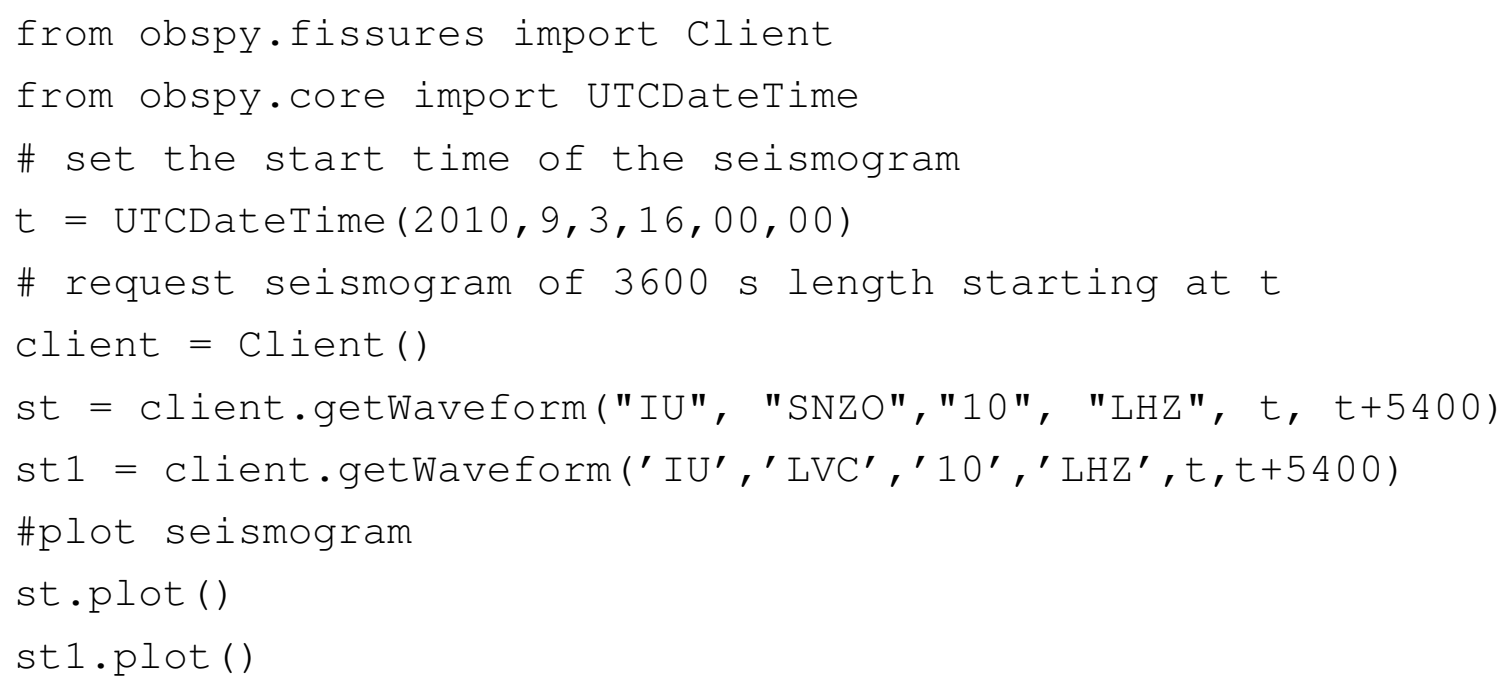

ObsPy makes extensive use of Python's foreign function library ctypes ${ }^{16}$ to move timecritical code to compiled shared libraries and to include open-source third-party libraries. To ensure continuation and stability, ObsPy's development is test-driven, that is, there is at least one test for every functionality in the ObsPy library. Furthermore, ObsPy is platform independent (it runs on Microsoft Windows, Macintosh Operating Systems and Linux) and its modular architecture allows adding new functionalities easily.

\footnotetext{
${ }^{15}$ IRIS: http://www.iris.edu

${ }^{16}$ ctypes: http://python.net/crew/theller/ctypes/
} 

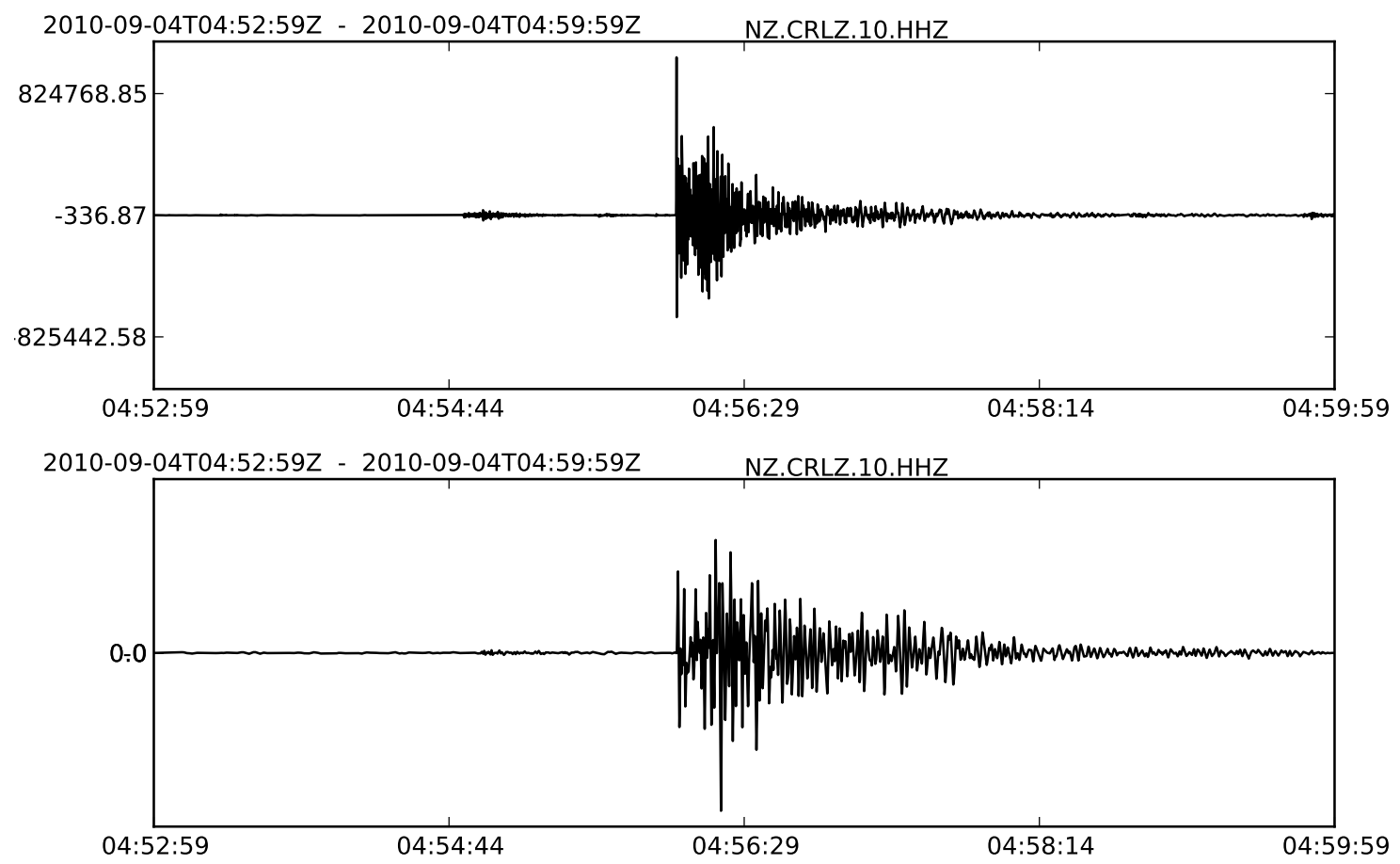

Figure A.4 Earthquake recorded at station CRLZ. The raw trace is shown on the top panel and the bottom panel shows the same trace after correcting for the instrument response, downsampling and applying a lowpass filter at $1 \mathrm{~Hz}$.
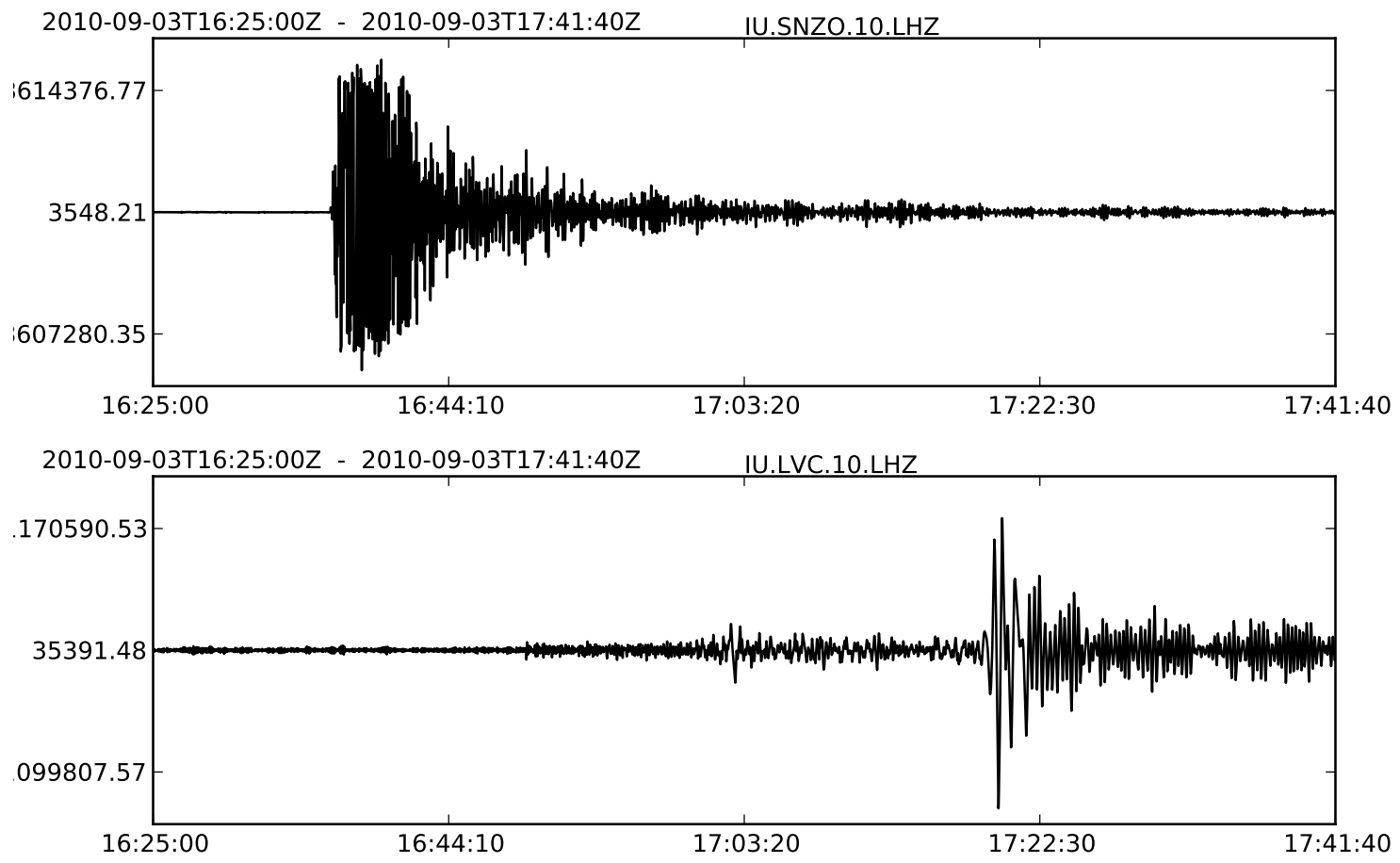

Figure A.5 The Darfield earthquake, close to Christchurch, New Zealand, recorded at the GSN station SNZO (South Karori, New Zealand) and LVC (Limon Verde, Chile) on 3 September 2010 between 16:25 and 17:41 UTC. 


\section{B. Response function database}

As discussed in Section 5.3.1, some of the stations from temporary deployments were found to have missing or apparently incorrect instrument response functions. We therefore compiled a list of instrument response information, used in this study, for stations not normally archived by any data management center. The listed response functions are for ground displacement in the form of poles and zeros, and the values are given in Rad. It has not been possible to unequivocally verify those parameters but document them here so that later studies can refer and, if necessary, correct them. For a comprehensive discussion on the topic of instrument response functions see Scherbaum [2007].

\section{B.1. CNIPSE}

\begin{tabular}{|l|l|l|l|}
\hline \multicolumn{3}{|c|}{ LKOW (BHZ, BHE, BHN) CMG-3T } \\
\hline \hline \multirow{4}{*}{ Poles } & $-1005.3100+0.0000 \mathrm{j}$ & & $0.0000+0.0000 \mathrm{j}$ \\
& $-502.6548+0.0000 \mathrm{j}$ & & $0.0000+0.0000 \mathrm{j}$ \\
& $-1130.9730+0.0000 \mathrm{j}$ & Zeros & $0.0000+0.0000 \mathrm{j}$ \\
& $-0.0370+0.0370 \mathrm{j}$ & & \\
& $-0.0370+-0.0370 \mathrm{j}$ & & \\
\hline
\end{tabular}

\begin{tabular}{|l|l|l|l|}
\hline \multicolumn{3}{|c|}{ LOTA (BHZ, BHE, BHN) CMG-3T } \\
\hline \hline \multirow{4}{*}{ Poles } & $-1005.3100+0.0000 \mathrm{j}$ & & $0.0000+0.0000 \mathrm{j}$ \\
& $-502.6548+0.0000 \mathrm{j}$ & & $0.0000+0.0000 \mathrm{j}$ \\
& $-1130.9730+0.0000 \mathrm{j}$ & Zeros & $0.0000+0.0000 \mathrm{j}$ \\
& $-0.0370+0.0370 \mathrm{j}$ & & \\
& $-0.0370+-0.0370 \mathrm{j}$ & & \\
\hline
\end{tabular}




\begin{tabular}{|l|l|l|l|}
\hline \multicolumn{3}{|c|}{ LTAT (BHZ, BHE, BHN) CMG-3T } \\
\hline \hline \multirow{4}{*}{ Poles } & $-0.0370+0.0370 \mathrm{j}$ & & $920.4870+0.0000 \mathrm{j}$ \\
& $-0.0370+-0.0370 \mathrm{j}$ & Zeros & $0.0000+0.0000 \mathrm{j}$ \\
& $-459.9290+236.2480 \mathrm{j}$ & & $0.0000+0.0000 \mathrm{j}$ \\
& $-459.9290+-236.2480 \mathrm{j}$ & & $0.0000+0.0000 \mathrm{j}$ \\
\hline
\end{tabular}

\begin{tabular}{|l|l|l|l|}
\hline \multicolumn{3}{|c|}{ LDEN (BHZ, BHE, BHN) CMG-3T } \\
\hline \hline \multirow{5}{*}{ Poles } & $-0.0370+0.0370 \mathrm{j}$ & & $0.0000+0.0000 \mathrm{j}$ \\
& $-0.0370+-0.0370 \mathrm{j}$ & & $0.0000+0.0000 \mathrm{j}$ \\
& $-314.1593+0.0000 \mathrm{j}$ & Zeros & $0.0000+0.0000 \mathrm{j}$ \\
& $-1382.3010+0.0000 \mathrm{j}$ & & \\
& $-2199.1150+0.0000 \mathrm{j}$ & & \\
\hline
\end{tabular}

\begin{tabular}{|l|l|l|l|}
\hline \multicolumn{3}{|c|}{ LJAI (BHZ, BHE, BHN) CMG-3T } \\
\hline \hline \multirow{5}{*}{ Poles } & $-1005.3100+0.0000 \mathrm{j}$ & & $0.0000+0.0000 \mathrm{j}$ \\
& $-502.6548+0.0000 \mathrm{j}$ & & $0.0000+0.0000 \mathrm{j}$ \\
& $-1130.9730+0.0000 \mathrm{j}$ & Zeros & $0.0000+0.0000 \mathrm{j}$ \\
& $-0.0370+0.0370 \mathrm{j}$ & & \\
& $-0.0370+-0.0370 \mathrm{j}$ & & \\
\hline
\end{tabular}

\begin{tabular}{|l|l|l|l|}
\hline \multicolumn{3}{|c|}{ LPOR (BHZ, BHE, BHN) CMG-3T } \\
\hline \hline \multirow{5}{*}{ Poles } & $-1005.3100+0.0000 \mathrm{j}$ & & $0.0000+0.0000 \mathrm{j}$ \\
& $-502.6548+0.0000 \mathrm{j}$ & & $0.0000+0.0000 \mathrm{j}$ \\
& $-1130.9730+0.0000 \mathrm{j}$ & Zeros & $0.0000+0.0000 \mathrm{j}$ \\
& $-0.0370+0.0370 \mathrm{j}$ & & \\
& $-0.0370+-0.0370 \mathrm{j}$ & & \\
\hline
\end{tabular}

\begin{tabular}{|l|l|l|l|}
\hline \multicolumn{3}{|c|}{ LCRO (BHZ, BHE, BHN) CMG-3T } \\
\hline \hline \multirow{5}{*}{ Poles } & $-1005.3100+0.0000 \mathrm{j}$ & & $0.0000+0.0000 \mathrm{j}$ \\
& $-502.6548+0.0000 \mathrm{j}$ & & $0.0000+0.0000 \mathrm{j}$ \\
& $-1130.9730+0.0000 \mathrm{j}$ & Zeros & $0.0000+0.0000 \mathrm{j}$ \\
& $-0.0370+0.0370 \mathrm{j}$ & & \\
& $-0.0370+-0.0370 \mathrm{j}$ & & \\
\hline
\end{tabular}




\begin{tabular}{|l|l|l|l|}
\hline \multicolumn{3}{|c|}{ LGDS (BHZ, BHE, BHN) CMG-3T } \\
\hline \hline \multirow{5}{*}{ Poles } & $-0.0370+0.0370 \mathrm{j}$ & & $0.0000+0.0000 \mathrm{j}$ \\
& $-0.0370+-0.0370 \mathrm{j}$ & & $0.0000+0.0000 \mathrm{j}$ \\
& $-314.1593+0.0000 \mathrm{j}$ & Zeros & $0.0000+0.0000 \mathrm{j}$ \\
& $-1382.3010+0.0000 \mathrm{j}$ & & \\
& $-2199.1150+0.0000 \mathrm{j}$ & & \\
\hline
\end{tabular}

\begin{tabular}{|l|l|l|l|}
\hline \multicolumn{3}{|c|}{ LOPO (BHZ, BHE, BHN) CMG-3T } \\
\hline \hline \multirow{4}{*}{ Poles } & $-0.0370+0.0370 \mathrm{j}$ & & $0.0000+0.0000 \mathrm{j}$ \\
& $-0.0370+-0.0370 \mathrm{j}$ & & $0.0000+0.0000 \mathrm{j}$ \\
& $-314.1593+0.0000 \mathrm{j}$ & Zeros & $0.0000+0.0000 \mathrm{j}$ \\
& $-1382.3010+0.0000 \mathrm{j}$ & & \\
& $-2199.1150+0.0000 \mathrm{j}$ & & \\
\hline
\end{tabular}

\begin{tabular}{|l|l|l|l|}
\hline \multicolumn{3}{|c|}{ WAIC (BHZ, BHE, BHN) CMG-40T } \\
\hline \hline \multirow{5}{*}{ Poles } & $-0.1480+0.1480 \mathrm{j}$ & & $-999.0260+0.0000 \mathrm{j}$ \\
& $-0.1480+-0.1480 \mathrm{j}$ & & $0.0000+0.0000 \mathrm{j}$ \\
& $-314.1600+0.0000 \mathrm{j}$ & Zeros & $0.0000+0.0000 \mathrm{j}$ \\
& $-9904.8000+3786.0000 \mathrm{j}$ & & $0.0000+0.0000 \mathrm{j}$ \\
& $-9904.8000+-3786.0000 \mathrm{j}$ & & \\
& $-12507.0000+0.0000 \mathrm{j}$ & & \\
\hline
\end{tabular}

\begin{tabular}{|l|l|l|l|}
\hline \multicolumn{3}{|c|}{ KIWC (BHZ, BHE, BHN) CMG-40T } \\
\hline \hline \multirow{5}{*}{ Poles } & $-0.1480+0.1480 \mathrm{j}$ & & $-999.0260+0.0000 \mathrm{j}$ \\
& $-0.1480+-0.1480 \mathrm{j}$ & & $0.0000+0.0000 \mathrm{j}$ \\
& $-314.1600+0.0000 \mathrm{j}$ & Zeros & $0.0000+0.0000 \mathrm{j}$ \\
& $-9904.8000+3786.0000 \mathrm{j}$ & & $0.0000+0.0000 \mathrm{j}$ \\
& $-9904.8000+-3786.0000 \mathrm{j}$ & & \\
& $-12507.0000+0.0000 \mathrm{j}$ & & \\
\hline
\end{tabular}

\begin{tabular}{|l|l|l|l|}
\hline \multicolumn{3}{|c|}{ RTKC (BHZ) CMG-40T } \\
\hline \hline \multirow{5}{*}{ Poles } & $-1005.3100+0.0000 \mathrm{j}$ & & $0.0000+0.0000 \mathrm{j}$ \\
& $-502.6548+0.0000 \mathrm{j}$ & & $0.0000+0.0000 \mathrm{j}$ \\
& $-1130.9730+0.0000 \mathrm{j}$ & Zeros & $0.0000+0.0000 \mathrm{j}$ \\
& $-0.1486+0.1486 \mathrm{j}$ & & \\
& $-0.1486+-0.1486 \mathrm{j}$ & & \\
\hline
\end{tabular}




\begin{tabular}{|l|l|l|l|}
\hline \multicolumn{3}{|c|}{ RTKC (BHE, BHN) } \\
\hline \hline \multirow{5}{*}{ Poles } & $-0.1480+0.1480 \mathrm{j}$ & & $-999.0260+0.0000 \mathrm{j}$ \\
& $-0.1480+-0.1480 \mathrm{j}$ & & $0.0000+0.0000 \mathrm{j}$ \\
& $-314.1600+0.0000 \mathrm{j}$ & Zeros & $0.0000+0.0000 \mathrm{j}$ \\
& $-9904.8000+3786.0000 \mathrm{j}$ & & $0.0000+0.0000 \mathrm{j}$ \\
& $-9904.8000+-3786.0000 \mathrm{j}$ & & \\
& $-12507.0000+0.0000 \mathrm{j}$ & & \\
\hline
\end{tabular}

\begin{tabular}{|l|l|l|l|}
\hline \multicolumn{3}{|c|}{ LPAP (BHZ, BHE, BHN) CMG-3T } \\
\hline \hline \multirow{4}{*}{ Poles } & $-0.0370+0.0370 \mathrm{j}$ & & $0.0000+0.0000 \mathrm{j}$ \\
& $-0.0370+-0.0370 \mathrm{j}$ & & $0.0000+0.0000 \mathrm{j}$ \\
& $-314.1593+0.0000 \mathrm{j}$ & Zeros & $0.0000+0.0000 \mathrm{j}$ \\
& $-1382.3010+0.0000 \mathrm{j}$ & & \\
& $-2199.1150+0.0000 \mathrm{j}$ & & \\
\hline
\end{tabular}

\begin{tabular}{|l|l|l|l|}
\hline \multicolumn{3}{|c|}{ LWAI (BHZ, BHE, BHN) CMG-40T } \\
\hline \hline \multirow{5}{*}{ Poles } & $-1005.3100+0.0000 \mathrm{j}$ & & $0.0000+0.0000 \mathrm{j}$ \\
& $-502.6548+0.0000 \mathrm{j}$ & & $0.0000+0.0000 \mathrm{j}$ \\
& $-1130.9730+0.0000 \mathrm{j}$ & Zeros & $0.0000+0.0000 \mathrm{j}$ \\
& $-0.1486+0.1486 \mathrm{j}$ & & \\
& $-0.1486+-0.1486 \mathrm{j}$ & & \\
\hline
\end{tabular}

\begin{tabular}{|l|l|l|l|}
\hline \multicolumn{3}{|c|}{ LMOW (BHZ, BHE, BHN) CMG-3T } \\
\hline \hline \multirow{3}{*}{ Poles } & $-0.9238+0.9514 \mathrm{j}$ & \multirow{2}{*}{ Zeros } & $0.0000+0.0000 \mathrm{j}$ \\
& $-0.9238+-0.9514 \mathrm{j}$ & & $0.0000+0.0000 \mathrm{j}$ \\
& & & $0.0000+0.0000 \mathrm{j}$ \\
\hline
\end{tabular}

\begin{tabular}{|l|l|l|l|}
\hline \multicolumn{3}{|c|}{ KARC (BHZ, BHE, BHN) CMG-40T } \\
\hline \hline \multirow{5}{*}{ Poles } & $-0.1480+0.1480 \mathrm{j}$ & & $-999.0260+0.0000 \mathrm{j}$ \\
& $-0.1480+-0.1480 \mathrm{j}$ & & $0.0000+0.0000 \mathrm{j}$ \\
& $-314.1600+0.0000 \mathrm{j}$ & Zeros & $0.0000+0.0000 \mathrm{j}$ \\
& $-9904.8000+3786.0000 \mathrm{j}$ & & $0.0000+0.0000 \mathrm{j}$ \\
& $-9904.8000+-3786.0000 \mathrm{j}$ & & \\
& $-12507.0000+0.0000 \mathrm{j}$ & & \\
\hline
\end{tabular}




\begin{tabular}{|l|l|l|l|}
\hline \multicolumn{3}{|c|}{ TUKC (BHZ, BHE, BHN) CMG-40T } \\
\hline \hline \multirow{5}{*}{ Poles } & $-0.1480+0.1480 \mathrm{j}$ & & $-999.0260+0.0000 \mathrm{j}$ \\
& $-0.1480+-0.1480 \mathrm{j}$ & & $0.0000+0.0000 \mathrm{j}$ \\
& $-314.1600+0.0000 \mathrm{j}$ & Zeros & $0.0000+0.0000 \mathrm{j}$ \\
& $-9904.8000+3786.0000 \mathrm{j}$ & & $0.0000+0.0000 \mathrm{j}$ \\
& $-9904.8000+-3786.0000 \mathrm{j}$ & & \\
& $-12507.0000+0.0000 \mathrm{j}$ & & \\
\hline
\end{tabular}

\begin{tabular}{|l|l|l|l|}
\hline \multicolumn{3}{|c|}{ POHC (BHZ, BHE, BHN) CMG-40T } \\
\hline \hline \multirow{5}{*}{ Poles } & $-0.1480+0.1480 \mathrm{j}$ & & $-999.0260+0.0000 \mathrm{j}$ \\
& $-0.1480+-0.1480 \mathrm{j}$ & & $0.0000+0.0000 \mathrm{j}$ \\
& $-314.1600+0.0000 \mathrm{j}$ & Zeros & $0.0000+0.0000 \mathrm{j}$ \\
& $-9904.8000+3786.0000 \mathrm{j}$ & & $0.0000+0.0000 \mathrm{j}$ \\
& $-9904.8000+-3786.0000 \mathrm{j}$ & & \\
& $-12507.0000+0.0000 \mathrm{j}$ & & \\
\hline
\end{tabular}

\begin{tabular}{|l|l|l|l|}
\hline \multicolumn{3}{|c|}{ KNGC (BHZ, BHE, BHN) CMG-40T } \\
\hline \hline \multirow{5}{*}{ Poles } & $-0.1480+0.1480 \mathrm{j}$ & & $-999.0260+0.0000 \mathrm{j}$ \\
& $-0.1480+-0.1480 \mathrm{j}$ & & $0.0000+0.0000 \mathrm{j}$ \\
& $-314.1600+0.0000 \mathrm{j}$ & Zeros & $0.0000+0.0000 \mathrm{j}$ \\
& $-9904.8000+3786.0000 \mathrm{j}$ & & $0.0000+0.0000 \mathrm{j}$ \\
& $-9904.8000+-3786.0000 \mathrm{j}$ & & \\
& $-12507.0000+0.0000 \mathrm{j}$ & & \\
\hline
\end{tabular}

\begin{tabular}{|l|l|l|l|}
\hline \multicolumn{3}{|c|}{ TAUC (BHZ, BHE, BHN) CMG-40T } \\
\hline \hline \multirow{5}{*}{ Poles } & $-0.1480+0.1480 \mathrm{j}$ & & $-999.0260+0.0000 \mathrm{j}$ \\
& $-0.1480+-0.1480 \mathrm{j}$ & & $0.0000+0.0000 \mathrm{j}$ \\
& $-314.1600+0.0000 \mathrm{j}$ & Zeros & $0.0000+0.0000 \mathrm{j}$ \\
& $-9904.8000+3786.0000 \mathrm{j}$ & & $0.0000+0.0000 \mathrm{j}$ \\
& $-9904.8000+-3786.0000 \mathrm{j}$ & & \\
& $-12507.0000+0.0000 \mathrm{j}$ & & \\
\hline
\end{tabular}

\begin{tabular}{|l|l|l|l|}
\hline \multicolumn{3}{|c|}{ LRAN (BHZ, BHE, BHN) CMG-3T } \\
\hline \hline \multirow{3}{*}{ Poles } & $-0.9238+0.9514 \mathrm{j}$ & Zeros & $0.0000+0.0000 \mathrm{j}$ \\
& $-0.9238+-0.9514 \mathrm{j}$ & & $0.0000+0.0000 \mathrm{j}$ \\
& & & $0.0000+0.0000 \mathrm{j}$ \\
\hline
\end{tabular}




\begin{tabular}{|l|l|l|l|}
\hline \multicolumn{3}{|c|}{ S16 (BHZ, BHE, BHN) CMG-40T } \\
\hline \hline \multirow{5}{*}{ Poles } & $-1005.3100+0.0000 \mathrm{j}$ & & $0.0000+0.0000 \mathrm{j}$ \\
& $-502.6548+0.0000 \mathrm{j}$ & & $0.0000+0.0000 \mathrm{j}$ \\
& $-1130.9730+0.0000 \mathrm{j}$ & Zeros & $0.0000+0.0000 \mathrm{j}$ \\
& $-0.1486+0.1486 \mathrm{j}$ & & \\
& $-0.1486+-0.1486 \mathrm{j}$ & & \\
\hline
\end{tabular}

\begin{tabular}{|l|l|l|l|}
\hline \multicolumn{3}{|c|}{ SCHC (BHZ, BHE, BHN) CMG-40T } \\
\hline \hline \multirow{5}{*}{ Poles } & $-0.1480+0.1480 \mathrm{j}$ & & $-999.0260+0.0000 \mathrm{j}$ \\
& $-0.1480+-0.1480 \mathrm{j}$ & & $0.0000+0.0000 \mathrm{j}$ \\
& $-314.1600+0.0000 \mathrm{j}$ & Zeros & $0.0000+0.0000 \mathrm{j}$ \\
& $-9904.8000+3786.0000 \mathrm{j}$ & & $0.0000+0.0000 \mathrm{j}$ \\
& $-9904.8000+-3786.0000 \mathrm{j}$ & & \\
& $-12507.0000+0.0000 \mathrm{j}$ & & \\
\hline
\end{tabular}

\begin{tabular}{|l|l|l|l|}
\hline \multicolumn{3}{|c|}{ LWTT (BHZ, BHE, BHN) CMG-3T } \\
\hline \hline \multirow{5}{*}{ Poles } & $-1005.3100+0.0000 \mathrm{j}$ & & $0.0000+0.0000 \mathrm{j}$ \\
& $-502.6548+0.0000 \mathrm{j}$ & & $0.0000+0.0000 \mathrm{j}$ \\
& $-1130.9730+0.0000 \mathrm{j}$ & Zeros & $0.0000+0.0000 \mathrm{j}$ \\
& $-0.0370+0.0370 \mathrm{j}$ & & \\
& $-0.0370+-0.0370 \mathrm{j}$ & & \\
\hline
\end{tabular}

\begin{tabular}{|l|l|l|l|}
\hline \multicolumn{3}{|c|}{ LGLS (BHZ, BHE, BHN) CMG-3T } \\
\hline \hline \multirow{5}{*}{ Poles } & $-0.0370+0.0370 \mathrm{j}$ & & $0.0000+0.0000 \mathrm{j}$ \\
& $-0.0370+-0.0370 \mathrm{j}$ & & $0.0000+0.0000 \mathrm{j}$ \\
& $-314.1593+0.0000 \mathrm{j}$ & Zeros & $0.0000+0.0000 \mathrm{j}$ \\
& $-1382.3010+0.0000 \mathrm{j}$ & & \\
& $-2199.1150+0.0000 \mathrm{j}$ & & \\
\hline
\end{tabular}

\begin{tabular}{|l|l|l|l|}
\hline \multicolumn{3}{|c|}{ TETC (BHZ, BHE, BHN) CMG-40T } \\
\hline \hline \multirow{5}{*}{ Poles } & $-0.1480+0.1480 \mathrm{j}$ & & $-999.0260+0.0000 \mathrm{j}$ \\
& $-0.1480+-0.1480 \mathrm{j}$ & & $0.0000+0.0000 \mathrm{j}$ \\
& $-314.1600+0.0000 \mathrm{j}$ & Zeros & $0.0000+0.0000 \mathrm{j}$ \\
& $-9904.8000+3786.0000 \mathrm{j}$ & & $0.0000+0.0000 \mathrm{j}$ \\
& $-9904.8000+-3786.0000 \mathrm{j}$ & & \\
& $-12507.0000+0.0000 \mathrm{j}$ & & \\
\hline
\end{tabular}




\begin{tabular}{|l|l|l|l|}
\hline \multicolumn{3}{|c|}{ LMAT (BHZ, BHE, BHN) CMG-3T } \\
\hline \hline \multirow{5}{*}{ Poles } & $-1005.3100+0.0000 \mathrm{j}$ & & $0.0000+0.0000 \mathrm{j}$ \\
& $-502.6548+0.0000 \mathrm{j}$ & & $0.0000+0.0000 \mathrm{j}$ \\
& $-1130.9730+0.0000 \mathrm{j}$ & Zeros & $0.0000+0.0000 \mathrm{j}$ \\
& $-0.0370+0.0370 \mathrm{j}$ & & \\
& $-0.0370+-0.0370 \mathrm{j}$ & & \\
\hline
\end{tabular}

\begin{tabular}{|l|l|l|l|}
\hline \multicolumn{3}{|c|}{ LMAU (BHZ, BHE, BHN) CMG-40T } \\
\hline \hline \multirow{5}{*}{ Poles } & $-1005.3100+0.0000 \mathrm{j}$ & & $0.0000+0.0000 \mathrm{j}$ \\
& $-502.6548+0.0000 \mathrm{j}$ & & $0.0000+0.0000 \mathrm{j}$ \\
& $-1130.9730+0.0000 \mathrm{j}$ & Zeros & $0.0000+0.0000 \mathrm{j}$ \\
& $-0.1486+0.1486 \mathrm{j}$ & & \\
& $-0.1486+-0.1486 \mathrm{j}$ & & \\
\hline
\end{tabular}

\begin{tabular}{|l|l|l|l|}
\hline \multicolumn{3}{|c|}{ LOCC (BHZ, BHE, BHN) CMG-40T } \\
\hline \hline \multirow{5}{*}{ Poles } & $-0.1480+0.1480 \mathrm{j}$ & & $-999.0260+0.0000 \mathrm{j}$ \\
& $-0.1480+-0.1480 \mathrm{j}$ & & $0.0000+0.0000 \mathrm{j}$ \\
& $-314.1600+0.0000 \mathrm{j}$ & Zeros & $0.0000+0.0000 \mathrm{j}$ \\
& $-9904.8000+3786.0000 \mathrm{j}$ & & $0.0000+0.0000 \mathrm{j}$ \\
& $-9904.8000+-3786.0000 \mathrm{j}$ & & \\
& $-12507.0000+0.0000 \mathrm{j}$ & & \\
\hline
\end{tabular}

\begin{tabular}{|l|l|l|l|}
\hline \multicolumn{3}{|c|}{ S25 (BHZ, BHE, BHN) CMG-3T } \\
\hline \hline \multirow{5}{*}{ Poles } & $-1005.3100+0.0000 \mathrm{j}$ & & $0.0000+0.0000 \mathrm{j}$ \\
& $-502.6548+0.0000 \mathrm{j}$ & & $0.0000+0.0000 \mathrm{j}$ \\
& $-1130.9730+0.0000 \mathrm{j}$ & Zeros & $0.0000+0.0000 \mathrm{j}$ \\
& $-0.0370+0.0370 \mathrm{j}$ & & \\
& $-0.0370+-0.0370 \mathrm{j}$ & & \\
\hline
\end{tabular}

\begin{tabular}{|l|l|l|l|}
\hline \multicolumn{3}{|c|}{ MILC (BHZ, BHE, BHN) CMG-40T } \\
\hline \hline \multirow{5}{*}{ Poles } & $-0.1480+0.1480 \mathrm{j}$ & & $-999.0260+0.0000 \mathrm{j}$ \\
& $-0.1480+-0.1480 \mathrm{j}$ & & $0.0000+0.0000 \mathrm{j}$ \\
& $-314.1600+0.0000 \mathrm{j}$ & Zeros & $0.0000+0.0000 \mathrm{j}$ \\
& $-9904.8000+3786.0000 \mathrm{j}$ & & $0.0000+0.0000 \mathrm{j}$ \\
& $-9904.8000+-3786.0000 \mathrm{j}$ & & \\
& $-12507.0000+0.0000 \mathrm{j}$ & & \\
\hline
\end{tabular}




\begin{tabular}{|l|l|l|l|}
\hline \multicolumn{3}{|c|}{ YUPC (BHZ, BHE, BHN) CMG-40T } \\
\hline \hline \multirow{5}{*}{ Poles } & $-0.1480+0.1480 \mathrm{j}$ & & $-999.0260+0.0000 \mathrm{j}$ \\
& $-0.1480+-0.1480 \mathrm{j}$ & & $0.0000+0.0000 \mathrm{j}$ \\
& $-314.1600+0.0000 \mathrm{j}$ & Zeros & $0.0000+0.0000 \mathrm{j}$ \\
& $-9904.8000+3786.0000 \mathrm{j}$ & & $0.0000+0.0000 \mathrm{j}$ \\
& $-9904.8000+-3786.0000 \mathrm{j}$ & & \\
& $-12507.0000+0.0000 \mathrm{j}$ & & \\
\hline
\end{tabular}

\section{B.2. WCNIPSE}

\begin{tabular}{|l|l|l|l|}
\hline \multicolumn{3}{|c|}{ PUKC (BHZ, BHE, BHN) CMG-40T } \\
\hline \hline \multirow{5}{*}{ Poles } & $-0.1480+0.1480 \mathrm{j}$ & & $999.0260+0.0000 \mathrm{j}$ \\
& $-0.1480+-0.1480 \mathrm{j}$ & & $0.0000+0.0000 \mathrm{j}$ \\
& $-314.1600+0.0000 \mathrm{j}$ & Zeros & $0.0000+0.0000 \mathrm{j}$ \\
& $-9904.8000+3786.0000 \mathrm{j}$ & & $0.0000+0.0000 \mathrm{j}$ \\
& $-9904.8000+-3786.0000 \mathrm{j}$ & & \\
& $-12507.0000+0.0000 \mathrm{j}$ & & \\
\hline
\end{tabular}

\begin{tabular}{|l|l|l|l|}
\hline \multicolumn{3}{|c|}{ STEC (BHZ, BHE, BHN) L4C-3D } \\
\hline \hline \multirow{5}{*}{ Poles } & $-0.1480+0.1480 \mathrm{j}$ & & $999.0260+0.0000 \mathrm{j}$ \\
& $-0.1480+-0.1480 \mathrm{j}$ & & $0.0000+0.0000 \mathrm{j}$ \\
& $-314.1600+0.0000 \mathrm{j}$ & Zeros & $0.0000+0.0000 \mathrm{j}$ \\
& $-9904.8000+3786.0000 \mathrm{j}$ & & $0.0000+0.0000 \mathrm{j}$ \\
& $-9904.8000+-3786.0000 \mathrm{j}$ & & \\
& $-12507.0000+0.0000 \mathrm{j}$ & & \\
\hline
\end{tabular}

\begin{tabular}{|l|l|l|l|}
\hline \multicolumn{3}{|c|}{ VERC (BHZ, BHE, BHN) CMG-40T } \\
\hline \hline \multirow{5}{*}{ Poles } & $-0.0740+-0.0740 \mathrm{j}$ & & $879.6460+0.0000 \mathrm{j}$ \\
& $-0.0740+0.0740 \mathrm{j}$ & & $0.0000+0.0000 \mathrm{j}$ \\
& $-304.1060+0.0000 \mathrm{j}$ & Zeros & $0.0000+0.0000 \mathrm{j}$ \\
& $-9904.8000+3786.0000 \mathrm{j}$ & & $0.0000+0.0000 \mathrm{j}$ \\
& $-9904.8000+-3786.0000 \mathrm{j}$ & & \\
& $-12507.0000+0.0000 \mathrm{j}$ & & \\
\hline
\end{tabular}




\begin{tabular}{|l|l|l|l|}
\hline \multicolumn{3}{|c|}{ WULC (BHZ, BHE, BHN) CMG-40T } \\
\hline \hline \multirow{5}{*}{ Poles } & $-0.1480+0.1480 \mathrm{j}$ & & $-999.0260+0.0000 \mathrm{j}$ \\
& $-0.1480+-0.1480 \mathrm{j}$ & & $0.0000+0.0000 \mathrm{j}$ \\
& $-314.1600+0.0000 \mathrm{j}$ & Zeros & $0.0000+0.0000 \mathrm{j}$ \\
& $-9904.8000+3786.0000 \mathrm{j}$ & & $0.0000+0.0000 \mathrm{j}$ \\
& $-9904.8000+-3786.0000 \mathrm{j}$ & & \\
& $-12507.0000+0.0000 \mathrm{j}$ & & \\
\hline
\end{tabular}

\begin{tabular}{|l|l|l|l|}
\hline \multicolumn{3}{|l|}{ YTFC (11/2004-1/2005) (BHZ, BHE, BHN) L4C-3D } \\
\hline \hline \multirow{5}{*}{ Poles } & $-0.0740+-0.0740 \mathrm{j}$ & & $879.6460+0.0000 \mathrm{j}$ \\
& $-0.0740+0.0740 \mathrm{j}$ & & $0.0000+0.0000 \mathrm{j}$ \\
& $-304.1060+0.0000 \mathrm{j}$ & Zeros & $0.0000+0.0000 \mathrm{j}$ \\
& $-9904.8000+3786.0000 \mathrm{j}$ & & $0.0000+0.0000 \mathrm{j}$ \\
& $-9904.8000+-3786.0000 \mathrm{j}$ & & \\
& $-12507.0000+0.0000 \mathrm{j}$ & & \\
\hline
\end{tabular}

\begin{tabular}{|l|l|l|l|}
\hline \multicolumn{3}{|c|}{ YTFC (7/2005-10/2005) (BHZ, BHE, BHN) CMG-40T } \\
\hline \hline \multirow{5}{*}{ Poles } & $-0.1480+-0.1480 \mathrm{j}$ & & $99.0260+0.0000 \mathrm{j}$ \\
& $-0.1480+0.1480 \mathrm{j}$ & & $0.0000+0.0000 \mathrm{j}$ \\
& $-314.1600+0.0000 \mathrm{j}$ & Zeros & $0.0000+0.0000 \mathrm{j}$ \\
& $-9904.8000+3786.0000 \mathrm{j}$ & & $0.0000+0.0000 \mathrm{j}$ \\
& $-9904.8000+-3786.0000 \mathrm{j}$ & & \\
& $-12507.0000+0.0000 \mathrm{j}$ & & \\
\hline
\end{tabular}

\begin{tabular}{|l|l|l|l|}
\hline \multicolumn{3}{|c|}{ PEAC (BHZ, BHE, BHN) CMG40-T } \\
\hline \hline \multirow{5}{*}{ Poles } & $-0.0740+-0.0740 \mathrm{j}$ & & $879.6460+0.0000 \mathrm{j}$ \\
& $-0.0740+0.0740 \mathrm{j}$ & & $0.0000+0.0000 \mathrm{j}$ \\
& $-304.1060+0.0000 \mathrm{j}$ & Zeros & $0.0000+0.0000 \mathrm{j}$ \\
& $-9904.8000+3786.0000 \mathrm{j}$ & & $0.0000+0.0000 \mathrm{j}$ \\
& $-9904.8000+-3786.0000 \mathrm{j}$ & & \\
& $-12507.0000+0.0000 \mathrm{j}$ & & \\
\hline
\end{tabular}




\section{B.3. NORD}

\begin{tabular}{|l|l|l|l|}
\hline \multicolumn{3}{|c|}{ TIKO (BHZ, BHE, BHN) CMG-40T } \\
\hline \hline \multirow{5}{*}{ Poles } & $-0.1480+0.1480 \mathrm{j}$ & & $999.0260+0.0000 \mathrm{j}$ \\
& $-0.1480+-0.1480 \mathrm{j}$ & & $0.0000+0.0000 \mathrm{j}$ \\
& $-314.1600+0.0000 \mathrm{j}$ & Zeros & $0.0000+0.0000 \mathrm{j}$ \\
& $-9904.8000+3786.0000 \mathrm{j}$ & & $0.0000+0.0000 \mathrm{j}$ \\
& $-9904.8000+-3786.0000 \mathrm{j}$ & & \\
& $-12507.0000+0.0000 \mathrm{j}$ & & \\
\hline
\end{tabular}

\begin{tabular}{|l|l|l|l|}
\hline \multicolumn{3}{|c|}{ OUZV (BHZ, BHE, BHN) STS2 } \\
\hline \hline \multirow{5}{*}{ Poles } & $-0.0740+-0.0740 \mathrm{j}$ & & $879.6460+0.0000 \mathrm{j}$ \\
& $-0.0740+0.0740 \mathrm{j}$ & & $0.0000+0.0000 \mathrm{j}$ \\
& $-304.1060+0.0000 \mathrm{j}$ & Zeros & $0.0000+0.0000 \mathrm{j}$ \\
& $-9904.8000+3786.0000 \mathrm{j}$ & & $0.0000+0.0000 \mathrm{j}$ \\
& $-9904.8000+-3786.0000 \mathrm{j}$ & & \\
& $-12507.0000+0.0000 \mathrm{j}$ & & \\
\hline
\end{tabular}

\begin{tabular}{|l|l|l|l|}
\hline \multicolumn{3}{|c|}{ WCZV (BHZ, BHE, BHN) CMG-3ESP } \\
\hline \hline \multirow{5}{*}{ Poles } & $-0.0740+-0.0740 \mathrm{j}$ & & $879.6460+0.0000 \mathrm{j}$ \\
& $-0.0740+0.0740 \mathrm{j}$ & & $0.0000+0.0000 \mathrm{j}$ \\
& $-304.1060+0.0000 \mathrm{j}$ & Zeros & $0.0000+0.0000 \mathrm{j}$ \\
& $-9904.8000+3786.0000 \mathrm{j}$ & & $0.0000+0.0000 \mathrm{j}$ \\
& $-9904.8000+-3786.0000 \mathrm{j}$ & & \\
& $-12507.0000+0.0000 \mathrm{j}$ & & \\
\hline
\end{tabular}

\begin{tabular}{|l|l|l|l|}
\hline \multicolumn{3}{|c|}{ MATA (BHZ, BHE, BHN) CMG-40T } \\
\hline \hline \multirow{5}{*}{ Poles } & $-0.1480+0.1480 \mathrm{j}$ & & $999.0260+0.0000 \mathrm{j}$ \\
& $-0.1480+-0.1480 \mathrm{j}$ & & $0.0000+0.0000 \mathrm{j}$ \\
& $-314.1600+0.0000 \mathrm{j}$ & Zeros & $0.0000+0.0000 \mathrm{j}$ \\
& $-9904.8000+3786.0000 \mathrm{j}$ & & $0.0000+0.0000 \mathrm{j}$ \\
& $-9904.8000+-3786.0000 \mathrm{j}$ & & \\
& $-12507.0000+0.0000 \mathrm{j}$ & & \\
\hline
\end{tabular}




\section{B.4. RF2004}

\begin{tabular}{|l|l|l|l|}
\hline \multicolumn{3}{|c|}{ RF1 (BHZ) CMG-40T } \\
\hline \hline \multirow{5}{*}{ Poles } & $-0.1480+0.1480 \mathrm{j}$ & & $999.0260+0.0000 \mathrm{j}$ \\
& $-0.1480+-0.1480 \mathrm{j}$ & & $1.0000+0.0000 \mathrm{j}$ \\
& $-314.1600+0.0000 \mathrm{j}$ & & $-1.0000+0.0000 \mathrm{j}$ \\
& $-9904.8000+3786.0000 \mathrm{j}$ & Zeros & $0.0000+0.0000 \mathrm{j}$ \\
& $-9904.8000+-3786.0000 \mathrm{j}$ & & $0.0000+0.0000 \mathrm{j}$ \\
& $-12507.0000+0.0000 \mathrm{j}$ & & \\
& $1.0000+0.0000 \mathrm{j}$ & & \\
& $-0.9844+0.0000 \mathrm{j}$ & & \\
\hline
\end{tabular}

\begin{tabular}{|l|l|l|l|}
\hline \multicolumn{3}{|c|}{ RF2 (BHZ) CMG-40T } \\
\hline \hline \multirow{5}{*}{ Poles } & $-0.1480+0.1480 \mathrm{j}$ & & $999.0260+0.0000 \mathrm{j}$ \\
& $-0.1480+-0.1480 \mathrm{j}$ & & $1.0000+0.0000 \mathrm{j}$ \\
& $-314.1600+0.0000 \mathrm{j}$ & & $-1.0000+0.0000 \mathrm{j}$ \\
& $-9904.8000+3786.0000 \mathrm{j}$ & Zeros & $0.0000+0.0000 \mathrm{j}$ \\
& $-9904.8000+-3786.0000 \mathrm{j}$ & & $0.0000+0.0000 \mathrm{j}$ \\
& $-12507.0000+0.0000 \mathrm{j}$ & & \\
& $1.0000+0.0000 \mathrm{j}$ & & \\
& $-0.9844+0.0000 \mathrm{j}$ & & \\
\hline
\end{tabular}

\begin{tabular}{|l|l|l|l|}
\hline \multicolumn{3}{|c|}{ RF3 (BHZ) CMG-40T } \\
\hline \hline \multirow{5}{*}{ Poles } & $-0.1480+0.1480 \mathrm{j}$ & & $999.0260+0.0000 \mathrm{j}$ \\
& $-0.1480+-0.1480 \mathrm{j}$ & & $1.0000+0.0000 \mathrm{j}$ \\
& $-314.1600+0.0000 \mathrm{j}$ & & $-1.0000+0.0000 \mathrm{j}$ \\
& $-9904.8000+3786.0000 \mathrm{j}$ & \multirow{6}{*}{ Zeros } & $0.0000+0.0000 \mathrm{j}$ \\
& $-9904.8000+-3786.0000 \mathrm{j}$ & & $0.0000+0.0000 \mathrm{j}$ \\
& $-12507.0000+0.0000 \mathrm{j}$ & & \\
& $1.0000+0.0000 \mathrm{j}$ & & \\
& $-0.9844+0.0000 \mathrm{j}$ & & \\
\hline
\end{tabular}




\begin{tabular}{|l|l|l|l|}
\hline \multicolumn{3}{|c|}{ RF4 (BHZ) CMG-40T } \\
\hline \hline \multirow{5}{*}{ Poles } & $-0.1480+0.1480 \mathrm{j}$ & & $999.0260+0.0000 \mathrm{j}$ \\
& $-0.1480+-0.1480 \mathrm{j}$ & & $1.0000+0.0000 \mathrm{j}$ \\
& $-314.1600+0.0000 \mathrm{j}$ & & $-1.0000+0.0000 \mathrm{j}$ \\
& $-9904.8000+3786.0000 \mathrm{j}$ & Zeros & $0.0000+0.0000 \mathrm{j}$ \\
& $-9904.8000+-3786.0000 \mathrm{j}$ & & $0.0000+0.0000 \mathrm{j}$ \\
& $-12507.0000+0.0000 \mathrm{j}$ & & \\
& $1.0000+0.0000 \mathrm{j}$ & & \\
& $-0.9844+0.0000 \mathrm{j}$ & & \\
\hline
\end{tabular}

\begin{tabular}{|l|l|l|l|}
\hline \multicolumn{3}{|c|}{ RF5 (BHZ) CMG-40T } \\
\hline \hline \multirow{5}{*}{ Poles } & $-0.1480+0.1480 \mathrm{j}$ & & $999.0260+0.0000 \mathrm{j}$ \\
& $-0.1480+-0.1480 \mathrm{j}$ & & $1.0000+0.0000 \mathrm{j}$ \\
& $-314.1600+0.0000 \mathrm{j}$ & & $-1.0000+0.0000 \mathrm{j}$ \\
& $-9904.8000+3786.0000 \mathrm{j}$ & Zeros & $0.0000+0.0000 \mathrm{j}$ \\
& $-9904.8000+-3786.0000 \mathrm{j}$ & & $0.0000+0.0000 \mathrm{j}$ \\
& $-12507.0000+0.0000 \mathrm{j}$ & & \\
& $1.0000+0.0000 \mathrm{j}$ & & \\
& $-0.9844+0.0000 \mathrm{j}$ & & \\
\hline
\end{tabular}

\begin{tabular}{|l|l|l|l|}
\hline \multicolumn{3}{|c|}{ RF6 (BHZ) CMG-40T } \\
\hline \hline \multirow{5}{*}{ Poles } & $-0.1480+0.1480 \mathrm{j}$ & & $999.0260+0.0000 \mathrm{j}$ \\
& $-0.1480+-0.1480 \mathrm{j}$ & & $1.0000+0.0000 \mathrm{j}$ \\
& $-314.1600+0.0000 \mathrm{j}$ & & $-1.0000+0.0000 \mathrm{j}$ \\
& $-9904.8000+3786.0000 \mathrm{j}$ & Zeros & $0.0000+0.0000 \mathrm{j}$ \\
& $-9904.8000+-3786.0000 \mathrm{j}$ & & $0.0000+0.0000 \mathrm{j}$ \\
& $-12507.0000+0.0000 \mathrm{j}$ & & \\
& $1.0000+0.0000 \mathrm{j}$ & & \\
& $-0.9844+0.0000 \mathrm{j}$ & & \\
\hline
\end{tabular}




\begin{tabular}{|l|l|l|l|}
\hline \multicolumn{3}{|c|}{ RF7 (BHZ) CMG-40T } \\
\hline \hline \multirow{5}{*}{ Poles } & $-0.1480+0.1480 \mathrm{j}$ & & $999.0260+0.0000 \mathrm{j}$ \\
& $-0.1480+-0.1480 \mathrm{j}$ & & $1.0000+0.0000 \mathrm{j}$ \\
& $-314.1600+0.0000 \mathrm{j}$ & & $-1.0000+0.0000 \mathrm{j}$ \\
& $-9904.8000+3786.0000 \mathrm{j}$ & \multirow{6}{*}{ Zeros } & $0.0000+0.0000 \mathrm{j}$ \\
& $-9904.8000+-3786.0000 \mathrm{j}$ & & $0.0000+0.0000 \mathrm{j}$ \\
& $-12507.0000+0.0000 \mathrm{j}$ & & \\
& $1.0000+0.0000 \mathrm{j}$ & & \\
& $-0.9844+0.0000 \mathrm{j}$ & & \\
\hline
\end{tabular}

\section{B.5. GeoNet stations}

Based on apparent $180^{\circ}$ phase shifts in our cross-correlation results (Section 3.4.2), the vertical component of the following permanent seismic stations are inferred to have reversed polarity:

1. BFZ (Birch Farm)

2. BKZ (Black Stump Farm)

3. HIZ (Hauiti)

4. KHZ (Kahutara)

5. KNZ (Kokohu)

6. MQZ (McQueen's Valley)

7. ODZ (Otahua Downs)

8. OUZ (Omahuta)

9. QRZ (Quartz Range)

10. RPZ (Rata Peaks)

11. URZ (Urewera)

12. WHZ (Wether Hill Road)

13. WPVZ (Whakapapa) 
B. Response function database 


\section{Bibliography}

Adams, C. J., N. Mortimer, H. J. Campbell, and W. L. Griffin, Age and isotopic characterisation of metasedimentary rocks from the Torlesse Supergroup and Waipapa Group in the central North Island, New Zealand, New Zealand Journal of Geology and Geophysics, 52(2), 149-170, doi:10.1080/00288300909509883, 2009.

Aki, K., Space and Time Spectra of Stationary Stochastic Waves, with Special Reference to Microtremors, Bulletin of the Earthquake Research Institute, 35, 415-457, 1957.

Aki, K., and P. G. Richards, Quantitative Seismology, 2nd ed., 700 pp., University Science Books, 2002.

Anderson, H., D. Eberhart-Phillips, T. McEvily, F. Wu, and R. Uhrhammer, Southern Alps passive seismic experiment, Science Report 1997/21, Inst. Geol. Nuc. Sci., 1997.

Audoine, E., M. K. Savage, and K. Gledhill, Anisotropic structure under a back arc spreading region, the Taupo Volcanic Zone, New Zealand, Journal of Geophysical Research B: Solid Earth, 109(11), 1-21, 2004.

Backus, G. E., and J. F. Gilbert, Numerical Applications of a Formalism for Geophysical Inverse Problems, Geophysical Journal International, 13(1-3), 247-276, doi:10.1111/j. 1365-246X.1967.tb02159.x, 1967.

Backus, G. E., and J. F. Gilbert, The Resolving Power of Gross Earth Data, Geophysical Journal International, 16(2), 169-205, doi:10.1111/j.1365-246X.1968.tb00216.x, 1968.

Balfour, N. J., M. K. Savage, and J. Townend, Stress and crustal anisotropy in Marlborough, New Zealand: evidence for low fault strength and structure-controlled anisotropy, Geophysical Journal International, 163(3), 1073-1086, 2005.

Bannister, S., and A. Melhuish, Seismic Scattering and Reverberation, Kaingaroa Plateau, Taupo Volcanic Zone, New Zealand, New Zealand Journal of Geology and Geophysics, 40, 375-381, doi:10.1080/00288306.1997.9514768, 1997. 
Bannister, S., C. J. Bryan, and H. M. Bibby, Shear Wave Velocity Variation Across the Taupo Volcanic Zone, New Zealand, from Receiver Function Inversion, Geophysical Journal International, 159(1), 291-310, doi:10.1111/j.1365-246X.2004.02384.x, 2004.

Bannister, S., M. Reyners, G. Stuart, and M. K. Savage, Imaging the Hikurangi subduction zone, New Zealand, using teleseismic receiver functions: crustal fluids above the forearc mantle wedge, Geophysical Journal International, 169(2), 602-616, doi: 10.1111/j.1365-246X.2007.03345.x, 2007.

Barmin, M. P., M. H. Ritzwoller, and A. L. Levshin, A Fast and Reliable Method for Surface Wave Tomography, Pure and Applied Geophysics, 158, 1351-1375, 2001.

Bassin, C., G. Laske, and G. Masters, The Current Limits of Resolution for Surface Wave Tomography in North America, EOS Trans AGU, 81(F897), 2000.

Baur, J. R., P. R. King, T. A. Stern, and B. Leitner, Development and Seismic Geomorphology of a Miocene Slope Channel Megasystem, Offshore Taranaki Basin, New Zealand, in Society of Economic Paleontologists and Mineralogists Foundation 30th Annual research conference, Houston, Texas, 2010.

Behr, Y., M. K. Townend, and S. Bannister, New Zealand Surface Wave Velocity Maps and S-velocity Profiles from Ambient Seismic Noise Correlation, in Geosciences '08, Wellington, NZ, 2008.

Behr, Y., J. Townend, S. Bannister, and M. K. Savage, Shear Velocity Structure of the Northland Peninsula, New Zealand, Inferred from Ambient Noise Correlations, Journal of Geophysical Research, 115(B5), 1-12, doi:10.1029/2009JB006737, 2010.

Behr, Y., J. Townend, S. Bannister, and M. K. Savage, Crustal shear wave tomography of the Taupo Volcanic Zone, New Zealand, via ambient noise correlation between multiple three-component networks, Geochemistry Geophysics Geosystems, 12(3), 1-18, doi: 10.1029/2010GC003385, 2011.

Bensen, G. D., M. H. Ritzwoller, M. P. Barmin, A. L. Levshin, F.-C. Lin, M. P. Moschetti, N. M. Shapiro, and Y. Yang, Processing Seismic Ambient Noise Data to Obtain Reliable Broad-Band Surface Wave Dispersion Measurements, Geophysical Journal International, 169(3), 1239-1260, doi:10.1111/j.1365-246X.2007.03374.x, 2007.

Bensen, G. D., M. H. Ritzwoller, and N. M. Shapiro, Broadband ambient noise surface wave tomography across the United States, Journal of Geophysical Research, 113, doi: 10.1029/2007JB005248, 2008. 
Berger, J., Ambient Earth noise: A survey of the Global Seismographic Network, Journal of Geophysical Research, 109(B11), 1-10, doi:10.1029/2004JB003408, 2004.

Bertero, M., and P. Boccacci, A Simple Method for the Reduction of Boundary Effects in the Richardson-Lucy Approach to Image Deconvolution, Astronomy \& Astrophysics, 437, 369-374, doi:10.1051/0004-6361, 2005.

Beyreuther, M., R. Barsch, L. Krischer, T. Megies, Y. Behr, and J. Wassermann, ObsPy: A Python Toolbox for Seismology, Seismological Research Letters, 81(3), 530-533, doi:10.1785/gssrl.81.3.530, 2010.

Bibby, H. M., T. G. Caldwell, F. J. Davey, and T. H. Webb, Geophysical Evidence of the Structure of the Taupo Volcanic Zone and its Hydrothermal Circulation, Journal of Volcanology and Geothermal Research, 68, 29-58, 1995.

Björnsson, A., H. Eysteinsson, and M. Beblo, Crustal Formation and Magma Genesis Beneath Iceland: Magnetotelluric Constraints, Special Papers-Geological Society of America, 388, 665, 2005.

Boness, N. L., and M. D. Zoback, Stress-induced seismic velocity anisotropy and physical properties in the SAFOD Pilot Hole in Parkfield, CA, Geophysical Research Letters, 31(L14S17), 15-18, doi:10.1029/2003GL019020, 2004.

Bonnefoy-Claudet, S., F. Cotton, and P. Bard, The Nature of Noise Wavefield and its Applications for Site Effects Studies; A Literature Review, Earth-Science Reviews, 79, 205-227, doi:10.1016/j.earscirev.2006.07.004, 2006.

Boschi, L., Measures of resolution in global body wave tomography, Geophysical Research Letters, 30(19), 1-4, doi:10.1029/2003GL018222, 2003.

Brenguier, F., N. M. Shapiro, M. Campillo, A. Nercessian, and V. Ferrazzini, 3-D Surface Wave Tomography of the Piton de la Fournaise Volcano using Seismic Noise Correlations, Geophysical Research Letters, 34, doi:10.1029/2006GL028586, 2007.

Brenguier, F., M. Campillo, C. Hadziioannou, N. M. Shapiro, R. M. Nadeau, and E. Larose, Postseismic relaxation along the San Andreas fault at Parkfield from continuous seismological observations., Science, 321(5895), 1478-1481, doi:10.1126/science. $1160943,2008$.

Brisbourne, A. M., and G. W. Stuart, Shear-Wave Velocity Structure beneath North Island, New Zealand, from Rayleigh-Wave Interstation Phase Velocities, Geophysical Journal International, 133, 175-184, 1998. 
Brooks, L. A., J. Townend, P. Gerstoft, S. Bannister, and L. Carter, Fundamental and Higher-Mode Rayleigh Wave Characteristics of Ambient Seismic Noise in New Zealand, Geophysical Research Letters, 36, doi:10.1029/2009GL040434, 2009a.

Brooks, L. A., J. Townend, P. Gerstoft, S. Bannister, and L. Carter, Characterising the New Zealand ambient seismic noise field: an oceanographic interpretation of western North Island spectra and beamforming results, in Proceedings of ACOUSTICS 2009, Adelaide, Australia, 2009b.

Brown, J., A. Colling, D. Park, J. Phillips, D. Rothery, and J. Wright, Waves, Tides and Shallow-Water Processes, 187 pp., The Open University/Pergamon Press, 1989.

Bussat, S., S. Kugler, and A. Statoilhydro, Recording Noise - Estimating Shear-Wave Velocities: Feasibility of Offshore Ambient-Noise Surface-Wave Tomography (ANSWT) on a Reservoir Scale, in SEG Houston 2009 International Exposition and Annual Meeting, May 2010, pp. 1627-1631, 2009.

Chapman, C. H., A new method for computing synthetic seismograms, Geophysical Journal International, 54(3), 481-518, doi:10.1111/j.1365-246X.1978.tb05491.x, 1978.

Claerbout, J. F., Synthesis of a Layered Medium from its Acoustic Transmission Response, Geophysics, 33(2), 264-269, 1968.

Collins, J., P. Molnar, and A. Sheehan, MOANA : Marine Observations of Anisotropy Near Aotearoa, 2010.

Creager, K., CORAL, Seismological Research Letters, 68(2), 269-271, 1997.

Dahlen, F. A., and J. Tromp, Theoretical Global Seismology, 1025 pp., Princeton University Press, 1998.

Dahlen, F. A., and Y. Zhou, Surface-wave group-delay and attenuation kernels, Geophysical Journal International, 165(2), 545-554, doi:10.1111/j.1365-246X.2006.02913.x, 2006.

Debayle, E., Inversion of massive surface wave data sets: Model construction and resolution assessment, Journal of Geophysical Research, 109(B2), doi:10.1029/ 2003JB002652, 2004.

Derode, A., E. Larose, M. Tanter, J. De Rosny, A. Tourin, M. Campillo, and M. Fink, Recovering the Green's function from field-field correlations in an open scattering medium, Journal of the Acoustical Society of America, 113(6), 2973-2976, 2003. 
Di Giulio, G., C. Cornou, M. Ohrnberger, M. Wathelet, and A. Rovelli, Deriving wavefield characteristics and shear-velocity profiles from two-dimensional small-aperture arrays analysis of ambient vibrations in a small-size alluvial basin, Colfiorito, Italy, Bulletin of the Seismological Society of America, 96(5), 1915-1933, 2006.

Duclos, M., Insights on plate boundary deformation from seismic anisotropy in the New Zealand upper mantle, Ph.D. thesis, Victoria University of Wellington, New Zealand, 2005.

Dunkin, J. W., Computation of modal solutions in layered, elastic media at high frequencies, Bulletin of the Seismological Society of America, 55, 335-358, 1965.

Duvall, T. L., S. M. Jefferies, J. W. Harvey, and M. A. Pomerantz, Time-Distance Helioseismology, Nature, 362, 430-432, 1993.

Dziewonski, A., and D. Anderson, Preliminary Reference Earth Model, Physics of the Earth and Planetary Interior, 25, 297-356, 1981.

Dziewonski, A., S. Block, and M. Landisman, A Technique for the Analysis of Transient Seismic Signals, Bulletin of the Seismological Society of America, 59, 427-444, 1969.

Eberhart-Phillips, D., Three-Dimensional P and S Velocity Structure in the Coalinga Region, California, Journal of Geophysical Research, 95(B10), 15,343-15,363, doi: 10.1029/JB095iB10p15343, 1990.

Eberhart-Phillips, D., and S. Bannister, 3-D imaging of Marlborough, New Zealand, subducted plate and strike-slip fault systems, Geophysical Journal International, 182, 7396, doi:10.1111/j.1365-246X.2010.04621.x, 2010.

Eberhart-Phillips, D., M. Reyners, M. Chadwick, and J. M. Chiu, Crustal Heterogeneity and Subduction Processes: 3-D Vp, Vp/Vs and Q in the Southern North Island, New Zealand, Geophysical Journal International, 162(1), 270-288, 2005.

Endrun, B., T. Meier, S. Lebedev, M. Bohnhoff, G. Stavrakakis, and H.-P. Harjes, S velocity structure and radial anisotropy in the Aegean region from surface wave dispersion, Geophysical Journal International, 174(2), 593-616, doi:10.1111/j.1365-246X.2008. 03802.x, 2008.

Feng, C.-C., and T.-L. Teng, An Error Analysis of Frequency-Time Analysis, Bulletin of the Seismological Society of America, 73(1), 143-155, 1983. 
Feng, M., and M. An, Lithospheric structure of the Chinese mainland determined from joint inversion of regional and teleseismic Rayleigh-wave group velocities, Journal of Geophysical Research, 115(B6), 1-16, doi:10.1029/2008JB005787, 2010.

Field, B. D., and C. I. Uruski, Cretaceous-Cenozoic Geology and Petroleum Systems of the East Coast Region, New Zealand, Institute of Geological \& Nuclear Science Monograph, 19, 1997.

Forghani, F., and R. Snieder, Underestimation of body waves and feasibility of surfacewave reconstruction by seismic interferometry, The Leading Edge, 29(7), 790, doi: 10.1190/1.3462779, 2010.

Forsyth, D. W., The Early Structural Evolution and Anisotropy of the Oceanic Upper Mantle, Geophysical Journal International, 43(1), 103-162, doi:10.1111/j.1365-246X. 1975.tb00630.x, 1975.

Fowler, C. M. R., The Solid Earth, Cambridge University Press, 2005.

Friedrich, A., F. Krüger, and K. Klinge, Ocean-Generated Microseismic Noise Located with the Gräfenberg Array, Journal of Seismology, 2, 47-64, 1998.

Funnell, R. H., D. S. Chapman, R. G. Allis, and P. A. Armstrong, Thermal state of the Taranaki Basin, New Zealand, New Zealand Journal of Geophysical Research, 101, 25,197-25,215, 1996.

Gallego, A., R. M. Russo, D. Comte, V. I. Mocanu, R. E. Murdie, and J. C. Vandecar, Seismic noise tomography in the Chile ridge subduction region, Geophysical Journal International, pp. 1478-1492, doi:10.1111/j.1365-246X.2010.04691.x, 2010.

Gerst, A., and M. K. Savage, Seismic anisotropy beneath Ruapehu Volcano: a possible eruption forecasting tool, Science, 306, 1543-1547, 2004.

Gerstoft, P., and T. Tanimoto, A year of microseisms in southern California, Geophysical Research Letters, 34(L20304), 1-6, doi:10.1029/2007GL031091, 2007.

Gerstoft, P., M. C. Fehler, and K. Sabra, When Katrina hit California, Geophysical Research Letters, 33(17), 2006a.

Gerstoft, P., K. Sabra, P. Roux, W. A. Kuperman, and M. C. Fehler, Green's Functions Extraction and Surface-Wave Tomography from Microseisms in Southern California, Geophysics, 71(4), SI23-SI31, $2006 b$. 
Goforth, T., and E. Herrin, Phase-matched filters: application to the study of Love waves, Bulletin of the Seismological Society of America, 69(1), 27-44, 1979.

Gorman, R. M., K. R. Bryan, and A. K. Laing, Wave hindcast for the New Zealand region: nearshore validation and coastal wave climate, New Zealand Journal of Marine and Freshwater Research, 37(3), 567-588, 2003a.

Gorman, R. M., K. R. Bryan, and A. K. Laing, Wave hindcast for the New Zealand region: deep-water wave climate, New Zealand Journal of Marine and Freshwater Research, 37(3), 589-612, 2003b.

Gouédard, P., C. Cornou, and P. Roux, Phase-velocity dispersion curves and small-scale geophysics using noise correlation slantstack technique, Geophysical Journal International, 172(3), 971-981, doi:10.1111/j.1365-246X.2007.03654.x, 2008.

Goutorbe, B., F. Lucazeau, and A. Bonneville, Surface heat flow and the mantle contribution on the margins of Australia, Geochemistry Geophysics Geosystems, 9, doi: 10.1029/2007GC001924, 2008.

Greve, S., M. K. Savage, and S. Hofmann, Strong Variations in Seismic Anisotropy across the Hikurangi Subduction Zone, North Island, New Zealand, Tectonophysics, 462(1-4), 7-21, doi:10.1016/j.tecto.2007.07.011, 2008.

Grimwood, P., Data on the Edge for Environmental Decision Making and Research, in APAN, Queenstown, NZ, 2008.

Grimwood, P., Y. Behr, J. Townend, J. Hine, and M. K. Savage, New Zealand Seismograph Information Service - Enhanced Access to Seismographic Data for Research and Teaching, in American Geophysical Union Fall Meeting, San Francisco, 2008.

Gudmundsson, O., A. Khan, and P. Voss, Rayleigh-wave group-velocity of the Icelandic crust from correlation of ambient seismic noise, Geophysical Research Letters, 34(14), 1-5, doi:10.1029/2007GL030215, 2007.

Gutenberg, B., Dispersion und Extinktion von seismischen Oberflächenwellen und der Aufbau der obersten Erdschichten, Physikalische Zeitschrift, 25, 377-381, 1924.

Haines, A. J., Seismic wave velocities in the uppermost mantle beneath New Zealand, New Zealand Journal of Geology and Geophysics, 22, 245-257, 1979. 
Harmon, N., D. Forsyth, and S. Webb, Using Ambient Seismic Noise to Determine Short-Period Phase Velocities and Shallow Shear Velocities in Young Oceanic Lithosphere, Bulletin of the Seismological Society of America, 97(6), 2009-2023, doi: 10.1785/0120070050, 2007.

Harmon, N., C. A. Rychert, G. A. Abers, M. Salas De La Cruz, K. M. Fischer, and P. Gerstoft, Phase velocities from seismic noise using beamforming and cross correlation in Costa Rica and Nicaragua, Geophysical Research Letters, 35(19), 1-6, doi: 10.1029/2008GL035387, 2008.

Harmon, N., C. Rychert, and P. Gerstoft, Distribution of noise sources for seismic interferometry, Geophysical Journal International, 183(3), 1470-1484, doi:10.1111/j. 1365-246X.2010.04802.x, 2010.

Harrison, A., and R. S. White, Crustal Structure of the Taupo Volcanic Zone, New Zealand: Stretching and Igneous Intrusion, Geophysical Research Letters, 31(13), 1-4, doi:10.1029/2004GL019885, 2004.

Harrison, A., and R. S. White, Lithospheric Structure of an Active Backarc Basin: the Taupo Volcanic Zone, New Zealand, Geophysical Journal International, 167(2), 968990, doi:10.1111/j.1365-246X.2006.03166.x, 2006.

Heise, W., H. M. Bibby, T. G. Caldwell, S. Bannister, Y. Ogawa, S. Takakura, and T. Uchida, Melt Distribution Beneath a Young Continental Rift: The Taupo Volcanic Zone, New Zealand, Geophysical Research Letters, 34(14), 1-6, doi:10.1029/ 2007GL029629, 2007.

Heise, W., T. G. Caldwell, H. M. Bibby, and S. L. Bennie, Three-Dimensional Electrical Resistivity Image of Magma Beneath an Active Continental Rift, Taupo Volcanic Zone, New Zealand, Geophysical Research Letters, 37(10), 2-6, doi:10.1029/ 2010GL043110, 2010.

Heming, R. F., Patterns of Quaternary basaltic volcanism in northern North Island, New Zealand, New Zealand Journal of Geology and Geophysics, 23, 335-344, 1980.

Herrin, E., and T. Goforth, Phase-matched filters: application to the study of Rayleigh waves, Bulletin of the Seismological Society of America, 67(5), 1259-1275, 1977.

Herzer, R. H., Seismic stratigraphy of a buried volcanic arc, Northland, New Zealand and implications for Neogene subduction, Marine and Petroleum Geology, 12, 511-531, 1995. 
Hoke, L., and R. Sutherland, Mantle melting beneath New Zealand revealed by helium in gas discharges, Tech. rep., GNS Science, 1999.

Holt, W. E., and T. A. Stern, Subduction, platform subsidence, and foreland thrust loading: The late Tertiary development of Taranaki Basin, New Zealand, Tectonics, 13, 10681092, 1994.

Horspool, N. A., M. K. Savage, and S. Bannister, Implications for intraplate volcanism and back-arc deformation in northwestern New Zealand, from joint inversion of receiver functions and surface waves, Geophysical Journal International, 166, 14661483, doi:10.1111/j.1365-246X.2006.03016.x, 2006.

Houghton, B. F., C. J. N. Wilson, M. O. McWilliams, M. a. Lanphere, S. D. Weaver, R. M. Briggs, and M. S. Pringle, Chronology and dynamics of a large silicic magmatic system: Central Taupo Volcanic Zone, New Zealand, Geology, 23(1), 13, doi:10.1130/ 0091-7613(1995)023<0013:CADOAL>2.3.CO;2, 1995.

Huang, Y., C. Hawkesworth, P. van Calsteren, I. Smith, and P. Black, Melt generation models for the Auckland volcanic field, New Zealand: constraints from U-Th isotopes, Earth and Planetary Science Letters, 149, 67-84, 1997.

Huang, Z., Rayleigh wave tomography of China and adjacent regions, Journal of Geophysical Research, 108(B2), doi:10.1029/2001JB001696, 2003.

Isaac, M. J., R. H. Herzer, F. J. Brook, and B. W. Hayward, Cretaceous and Cenozoic sedimentary basins of Northland, New Zealand, Institute of Geological and Nuclear Sciences Monograph 8, 1994.

Johnson, D. H., and D. E. Dudgeon, Array Signal Processing: Concepts and Techniques, 132-137 pp., 1993.

Juliá, J., C. J. Ammon, R. B. Herrmann, and A. M. Correig, Joint inversion of receiver function and surface wave dispersion observations, Geophysical Journal International, 143, 99-112, 2000.

Kang, T. S., and J. S. Shin, Surface-wave tomography from ambient seismic noise of accelerograph networks in southern Korea, Geophysical Research Letters, 33(17), 2006.

Kedar, S., M. S. Longuet-Higgins, F. Webb, N. Graham, R. Clayton, and C. Jones, The origin of deep ocean microseisms in the North Atlantic Ocean, Proceedings of the Royal Society A: Mathematical, Physical and Engineering Sciences, 464(2091), 777793, doi:10.1098/rspa.2007.0277, 2008. 
Kibblewhite, A. C., and K. C. Ewans, Wave-wave interactions, microseisms, and infrasonic ambient noise in the ocean, The Journal of the Acoustical Society of America, 78(3), 981, doi:10.1121/1.392931, 1985.

King, P. R., and G. P. Thrasher, Cretaceous-Cenozoic geology and petroleum systems of the Taranaki Basin, New Zealand, Institute of Geological \& Nuclear Sciences monograph, 1996.

Kradolfer, U., Automating the Exchange of Earthquake Information, EOS Trans. Amer. Geophys. U., 74, 442,444-445, 1993.

Kradolfer, U., AutoDRM - The First Five Years, Seism. Res. Let., 67, 30-33, 1996.

Kuo, J., J. Brune, and M. Major, Rayleigh Wave Dispersion in the Pacific Ocean for the Period Range 20 to 140 Seconds, Bulletin of the Seismological Society of America, 52(2), 333-357, 1962.

Laing, A. K., New Zealand wave climate from satellite observations, New Zealand Journal of Marine and Freshwater Research, 34, 727-744, 2000.

Lamb, S. H., Tectonic rotations about vertical axes during the last $4 \mathrm{Ma}$ in part of the New Zealand plate-boundary zone, Journal of Structural Geology, 10(8), 875-893, 1988.

Larose, E., L. Margerin, A. Derode, B. van Tiggelen, M. Campillo, N. M. Shapiro, A. Paul, L. Stehly, and M. Tanter, Correlation of random wavefields: an interdisciplinary review, Geophysics, 71, S111-S121, doi:10.1190/1.2213356, 2006.

Lebedev, E. B., and N. I. Khitarov, Dependence on the beginning of melting of granite and the electrical conductivity of its melt on high water vapor pressure, Geochem. Int., 1, 193-197, 1964.

Lee, M. W., Spectral Whitening in the Frequency Domain, Open-File Report (USGS), 86-108, 1986.

Lévêque, J. J., L. Rivera, and G. Wittlinger, On the use of the checker-board test to assess the resolution of tomographic inversions, Geophysical Journal International, 115, 313318, 1993.

Lévêque, J. J., E. Debayle, and V. Maupin, Anisotropy in the Indian Ocean upper mantle from Rayleigh-and Love-waveform inversion, Geophysical Journal International, 133(3), 529-540, 1998. 
Levshin, A. L., and M. H. Ritzwoller, Automated Detection, Extraction, and Measurement of Regional Surface Waves, Pure and Applied Geophysics, 158, 1531-1545, 2001.

Levshin, A. L., V. F. Pisarenko, and G. A. Pogrebinsky, On a frequency-time analysis of oscillations, Annals of Geophysics, 28, 211-218, 1972.

Levshin, A. L., L. Ratnikova, and J. Berger, Peculiarities of surface-wave propagation across central Eurasia, Bulletin of the Seismological Society of America, 82(6), 24642493, 1992.

Li, H., F. Bernardi, and A. Michelini, Surface wave dispersion measurements from ambient seismic noise analysis in Italy, Geophysical Journal International, 180(3), 12421252, doi:10.1111/j.1365-246X.2009.04476.x, 2010.

Li, S., M. Unsworth, J. Booker, W. Wei, H. Tan, and A. Jones, Partial melt or aqueous fluid in the mid-crust of Southern Tibet? Constraints from INDEPTH magnetotelluric data, Geophysical Journal International, 153(2), 289-304, 2003.

Liang, C., and C. A. Langston, Three-dimensional crustal structure of eastern North America extracted from ambient noise, Journal of Geophysical Research, 114, doi: 10.1029/2008JB005919, 2009.

Lin, F.-C., and M. H. Ritzwoller, Empirically determined finite frequency sensitivity kernels for surface waves, Geophysical Journal International, 182(2), 923-932, doi: 10.1111/j.1365-246X.2010.04643.x, 2010.

Lin, F.-C., M. H. Ritzwoller, J. Townend, S. Bannister, and M. K. Savage, Ambient Noise Rayleigh Wave Tomography of New Zealand, Geophysical Journal International, 170, 649-666, doi:10.1111/j.1365-246X.2007.03414.x, 2007.

Lin, F.-C., M. P. Moschetti, and M. H. Ritzwoller, Surface wave tomography of the western United States from ambient seismic noise: Rayleigh and Love wave phase velocity maps, Geophysical Journal International, 173, 281-298, doi:10.1111/j.1365-246X. 2008.03720.x, 2008.

Liu, Y., A. T. Anderson, C. J. N. Wilson, A. M. Davis, and I. M. Steele, Mixing and differentiation in the Oruanui rhyolitic magma, Taupo, New Zealand: evidence from volatiles and trace elements in melt inclusions, Contributions to Mineralogy and Petrology, 151(1), 71-87, doi:10.1007/s00410-005-0046-3, 2005. 
Lobkis, O. I., and R. L. Weaver, On the emergence of the Green's function in the correlations of a diffuse field, Journal of the Acoustical Society of America, 110(6), 30113017, 2001.

Longuet-Higgins, M. S., A Theory of the Origin of Microseisms, Philosophical Transactions of the Royal Society A: Mathematical, Physical and Engineering Sciences, 243(857), 1-35, doi:10.1098/rsta.1950.0012, 1950.

Louie, J. N., Faster, Better: Shear-Wave Velocity to 100 Meters Depth from Refraction Microtremor Arrays, Bulletin of the Seismological Society of America, 91(2), 347364, doi:10.1785/0120000098, 2001.

Love, A. E. H., Some Problems of Geodynamics, Cambridge University Press, 1911.

Marone, F., Y. Gung, and B. Romanowicz, Three-dimensional radial anisotropic structure of the North American upper mantle from inversion of surface waveform data, Geophysical Journal International, 171(1), 206-222, doi:10.1111/j.1365-246X.2007. 03465.x, 2007.

Marson-Pidgeon, K., M. K. Savage, K. Gledhill, and G. Stuart, Seismic anisotropy beneath the lower half of the North Island, New Zealand, Journal of Geophysical Research B: Solid Earth, 104, 20,277-20,286, 1999.

McMechan, G. A., and M. J. Yedlin, Analysis of dispersive waves by wave field transformation, Geophysics, 46(6), 869-874, 1981.

Mitchell, B. J., On the inversion of Love- and Rayleigh-wave Dispersion and Implications for Earth Structure and Anisotropy, Geophysical Journal International, 76(1), 233-241, doi:10.1111/j.1365-246X.1984.tb05040.x, 1984.

Mocquet, A., B. Romanowicz, and J. Montagner, Three-dimensional structure of the upper mantle beneath the Atlantic Ocean inferred from long-period Rayleigh waves 1. Group and phase velocity distributions, Journal of Geophysical, 94(B6), 7449-7468, 1989.

Mokhtar, T. A., R. B. Herrmann, and D. R. Russel, Seismic velocity and Q model for the shallow structure of the Arabian shield from short-period Rayleigh waves, Geophysics, 53(11), 1379-1387, 1988.

Mortimer, N., New Zealand's Geological Foundations, Gondwana Research, 7(1), 261$272,2004$. 
Moschetti, M. P., M. H. Ritzwoller, F.-C. Lin, and Y. Yang, Seismic evidence for widespread western-US deep-crustal deformation caused by extension, Nature, 464(7290), 885-889, doi:10.1038/nature08951, 2010.

Nishida, K., H. Kawakatsu, and K. Obara, Three-dimensional crustal S wave velocity structure in Japan using microseismic data recorded by Hi-net tiltmeters, Journal of Geophysical Research, 113(B10302), 1-22, doi:10.1029/2007JB005395, 2008.

Nolet, G., R. Montelli, and J. Virieux, Explicit, approximate expressions for the resolution and a posteriori covariance of massive tomographic systems, Geophysical Journal International, 138(1), 36-44, doi:10.1046/j.1365-246x.1999.00858.x, 1999.

Pandey, O. P., Terrestrial heat flow in New Zealand, Ph.D. thesis, Victoria University of Wellington, 1981.

Park, C. B., Imaging dispersion curves of surface waves on multi-channel record, SEG Technical Program Expanded Abstracts, 17(1), 1377, doi:10.1190/1.1820161, 1999.

Parker, R. L., Geophysical Inverse Theory, Princeton University Press, 1994.

Paul, A., M. Campillo, L. Margerin, and E. Larose, Empirical Synthesis of TimeAsymmetrical Green Functions from the Correlation of Coda Waves, Journal of Geophysical Research, 110, 1-13, doi:10.1029/2004JB003521, 2005.

Peter, D., L. Boschi, F. Deschamps, B. Fry, G. Ekström, and D. Giardini, A new finitefrequency shear-velocity model of the European-Mediterranean region, Geophysical Research Letters, 35, doi:10.1029/2008GL034769, 2008.

Petersen, J., Observations and Modeling of Seismic Background Noise, USGS Open File Report, 93-322, 1993.

Pickrill, R. A., and J. S. Mitchell, Ocean wave characteristics around New Zealand, New Zealand Journal of Marine and Freshwater Research, 13, 501-520, 1979.

Poggi, V., and D. Fäh, Estimating Rayleigh wave particle motion from three-component array analysis of ambient vibrations, Geophysical Journal International, 180(1), 251267, doi:10.1111/j.1365-246X.2009.04402.x, 2010.

Press, W. H., S. A. Teukolsky, W. T. Vetterling, and B. P. Flannery, Numerical Recipes in C++: The Art of Scientific Computing, Cambridge University Press, 2005. 
Pulford, A., and T. A. Stern, Pliocene exhumation and landscape evolution of central North Island, New Zealand: The role of the upper mantle, Journal of Geophysical Research, 109(F1), F01,016, doi:10.1029/2003JF000046, 2004.

Rayleigh, J. W. S., On waves propagated along the plane surface of an elastic solid, Proceedings of the London Mathematical Society, 17, 4-11, 1885.

Reyners, M., Plate coupling and the hazard of large subduction thrust earthquakes at the Hikurangi subduction zone, New Zealand, New Zealand Journal of Geology and Geophysics, 41, 343-354, 1998.

Reyners, M., and D. Eberhart-Phillips, Small earthquakes provide insight into plate coupling and fluid distribution in the Hikurangi subduction zone, New Zealand, Earth and Planetary Science Letters, 282(1-4), 299-305, doi:10.1016/j.epsl.2009.03.034, 2009.

Reyners, M., and G. Stuart, The central North Island passive seismic experiment, Science Report 2002/11, Inst. Geol. Nuc. Sci., 2002.

Reyners, M., D. Eberhart-Phillips, and G. Stuart, A three-dimensional image of shallow subduction: crustal structure of the Raukumara Peninsula, New Zealand, Geophysical Journal International, 137(3), 873-890, 1999.

Reyners, M., D. Eberhart-Phillips, G. Stuart, and Y. Nishimura, Imaging subduction from the trench to $300 \mathrm{~km}$ depth beneath the central North Island, New Zealand, with Vp and Vp/Vs, Geophysical Journal International, 165(2), 565-583, doi:10.1111/j.1365-246X. 2006.02897.x, 2006.

Rhie, J., and B. Romanowicz, A study of the relation between ocean storms and the Earth's hum, Geochemistry Geophysics Geosystems, 7, 2006.

Rickett, J. E., and J. F. Claerbout, Calculation of the Sun's Acoustic Impulse Response by Multi-Dimensional Spectral Factorization, Solar Physics, 192, 203-210, 2000.

Ritzwoller, M. H., and A. L. Levshin, Eurasian surface wave tomography: group velocities, Journal of Geophysical Research, 103(B3), 4839-4878, 1998.

Ritzwoller, M. H., N. M. Shapiro, M. P. Barmin, and A. L. Levshin, Global Surface Wave Diffraction Tomography, Journal of Geophysical Research, 107, 2335, 2002.

Robinson, R., E. Smith, and J. Latter, Seismic studies of the crust under the hydrothermal areas of the Taupo Volcanic Zone, New Zealand, Journal of Volcanology and Geothermal Research, 9(2-3), 253-267, doi:10.1016/0377-0273(81)90007-X, 1981. 
Roueff, A., P. Roux, and P. Refregier, Wave Separation in Ambient Seismic Noise Using Intrinsic Coherence and Polarization Filtering, Signal Processing, 89(4), 410-421, doi: 10.1016/j.sigpro.2008.09.008, 2009.

Roux, P., K. Sabra, P. Gerstoft, and W. A. Kuperman, P-waves from cross-correlation of seismic noise, Geophysical Research Letters, 32, doi:10.1029/2005GL023803, 2005.

Rowland, J. V., C. J. N. Wilson, and D. M. Gravley, Spatial and temporal variations in magma-assisted rifting, Taupo Volcanic Zone, New Zealand, Journal of Volcanology and Geothermal Research, 190(1-2), 89-108, doi:10.1016/j.jvolgeores.2009.05.004, 2010.

Rowlands, D. P., R. S. White, and A. J. Haines, Seismic tomography of the Tongariro Volcanic Centre, New Zealand, Geophysical Journal International, 163(3), 1180-1194, doi:10.1111/j.1365-246X.2005.02716.x, 2005.

Sabra, K., P. Gerstoft, P. Roux, W. A. Kupermann, and M. C. Fehler, Extracting timedomain Green's function estimates from ambient seismic noise, Geophysical Research Letters, 32(3), 1-5, doi:10.1029/2004GL021862, 2005.

Sambridge, M., Geophysical inversion with the neighbourhood algorithm -I. Searching a parameter space, Geophysical Journal International, 138, 479-494, 1999a.

Sambridge, M., Geophysical inversion with the neighbourhood algorithm -II. Appraising the ensemble, Geophysical Journal International, 138, 727-746, 1999b.

Sambridge, M., Finding acceptable models in nonlinear inverse problems using a neighbourhood algorithm, Inverse Problems, 17, 387-403, 2001.

Sánchez-Sesma, F. J., and M. Campillo, Retrieval of the Green function from crosscorrelation: the canonical elastic problem, Bulletin of the Seismological Society of America, 96, 1182-1191, doi:10.1785/0120050181, 2006.

Satake, K., and T. Hashida, Three-dimensional attenuation structure beneath North Island, New Zealand, Tectonophysics, 159, 181-194, 1989.

Savage, M. K., Lower crustal anisotropy or dipping boundaries? Effects on receiver functions and a case study in New Zealand, Journal of Geophysical Research B: Solid Earth, 103(7), 15,069-15,087, 1998.

Savage, M. K., J. Park, and H. Todd, Velocity and anisotropy structure at the Hikurangi subduction margin, New Zealand from receiver functions, Geophysical Journal International, 168, 1034-1050, doi:10.1111/j.1365-246X.2006.03086.x, 2007. 
Savage, M. K., T. Ohminato, Y. Aoki, H. Tsuji, and S. M. Greve, Stress magnitude and its temporal variation at Mt. Asama Volcano, Japan, from seismic anisotropy and GPS, Earth and Planetary Science Letters, 290(3-4), 403-414, doi:10.1016/j.eps1.2009.12. 037, 2010.

Scherbaum, F., Of poles and zeros, 268 pp., Kluwer Academic Publishers, 2007.

Schmeling, H., Numerical models on the influence of partial melt on elastic, anelastic and electric properties of rocks. Part I: elasticity and anelasticity, Physics of The Earth and Planetary Interiors, 41(1), 34-57, doi:10.1016/0031-9201(85)90100-1, 1985.

Sens-Schoenfelder, C., Synchronizing seismic networks with ambient noise, Geophysical Journal International, 174, 966-970, doi:10.1111/j.1365-246X.2008.03842.x, 2008.

Seward, A. M., C. M. Henderson, and E. G. C. Smith, Models of the upper mantle beneath the central North Island, New Zealand, from speeds and anisotropy of subhorizontal $\mathrm{P}$ waves (Pn), Journal of Geophysical Research, 114, 2009.

Shane, P., V. C. Smith, and I. Nairn, Millennial timescale resolution of rhyolite magma recharge at Tarawera volcano: insights from quartz chemistry and melt inclusions, Contributions to Mineralogy and Petrology, 156(3), 397-411, doi:10.1007/ s00410-008-0292-2, 2008.

Shapiro, N. M., and M. Campillo, Emergence of broadband Rayleigh waves from correlations of the ambient seismic noise, Geophysical Research Letters, 31, 1-4, doi: 10.1029/2004GL019491, 2004.

Shapiro, N. M., M. H. Ritzwoller, P. Molnar, and V. Levin, Thinning and flow of Tibetan crust constrained by seismic anisotropy, Science, 305, 233-236, doi:10.1126/science. 1098276, 2004.

Shapiro, N. M., M. Campillo, L. Stehly, and M. H. Ritzwoller, High-resolution surfacewave tomography from ambient seismic noise., Science (New York, N.Y.), 307(5715), 1615-1618, doi:10.1126/science.1108339, 2005.

Sherburn, S., and R. S. White, Crustal seismicity in Taranaki, New Zealand using accurate hypocentres from a dense network, Geophysical Journal International, 162(2), 494 506, doi:10.1111/j.1365-246X.2005.02667.x, 2005.

Sherburn, S., R. S. White, and M. Chadwick, Three-dimensional tomographic imaging of the Taranaki volcanoes, New Zealand, Geophysical Journal International, 166(2), 957-969, doi:10.1111/j.1365-246X.2006.03040.x, 2006. 
Sibson, R., and J. Rowland, Stress, fluid pressure and structural permeability in seismogenic crust, North Island, New Zealand, Geophysical Journal International, 154(2), 584-594, 2003.

Sinclair, M. R., Extratropical transition of southwest Pacific tropical cyclones. Part I: climatology and mean structure changes, Monthly Weather Review, 130, 2002.

Smith, I. E. M., T. Okada, T. Itaya, and P. M. Black, Age relationships and tectonic implications of late Cenozoic basaltic volcanism in Northland, New Zealand, New Zealand Journal of Geology and Geophysics, 36, 385-393, 1993.

Snieder, R., Extracting the Green's function from the correlation of coda waves: a derivation based on stationary phase, Physical Review, 69, 46,610-46,611, doi:10.1103/ PhysRevE.69.046610, 2004.

Snieder, R., and K. Wapenaar, Imaging with ambient noise, Physics Today, (September), 44-49, 2010.

Snieder, R., E. Slob, and K. Wapenaar, Lagrangian Green's function extraction, with applications to potential fields, diffusion and acoustic waves, New Journal of Physics, $12,1-24,2010$.

Snoke, J. A., and M. Sambridge, Constraints on the $\mathrm{S}$ wave velocity structure in a continental shield from surface wave data: comparing linearized least squares inversion and the direct search Neighbourhood Algorithm, Journal of Geophysical Research, 107, doi:10.1029/2001JB000498, 2002.

Spörli, K. B., Geology of Northland: accretion, allochthons and arcs at the edge of the New Zealand micro-continent, chap. Tectonic F, pp. 3-14, The Royal Society of New Zealand, 1989.

Stachnik, J. C., K. Dueker, D. L. Schutt, and H. Yuan, Imaging Yellowstone plumelithosphere interactions from inversion of ballistic and diffusive Rayleigh wave dispersion and crustal thickness data, Geochemistry Geophysics Geosystems, 9(6), doi: 10.1029/2008GC001992, 2008

Stehly, L., M. Campillo, and N. M. Shapiro, A study of the seismic noise from its longrange correlation properties, Journal of Geophysical Research-Solid Earth, 111(B10), 2006 
Stehly, L., B. Fry, M. Campillo, N. M. Shapiro, J. Guilbert, L. Boschi, and D. Giardini, Tomography of the Alpine region from observations of seismic ambient noise, Geophysical Journal International, 178(1), 338-350, doi:10.1111/j.1365-246X.2009.04132.x, 2009.

Stein, S., and M. Wysession, An Introduction to Seismology, Earthquakes, and Earth Structure, Blackwell Publishing, 2003.

Stern, T. A., Subduction at a continental margin: kinematics and dynamics of the central North Island, New Zealand, Reviews of Geophysics, 2006.

Stern, T. A., and A. M. Benson, Wide-angle seismic imaging beneath an andesitic arc: central North Island, New Zealand, Journal of Geophysical Research, submitted, 2011.

Stern, T. A., E. G. C. Smith, F. J. Davey, and K. J. Muirhead, Crustal and upper mantle structure of the northwestern North Island, New Zealand, from seismic refraction data, Geophys. J. R. astr. Soc., 91, 913-936, 1987.

Stern, T. A., W. R. Stratford, and M. L. Salmon, Subduction evolution and mantle dynamics at a continental margin: Central North Island, New Zealand, Reviews of Geophysics, 44(4), doi:10.1029/2005RG000171, 2006.

Stratford, W. R., and T. A. Stern, Crust and upper mantle structure of a continental backarc: central North Island, New Zealand, Geophysical Journal International, 166, 469-484, doi:10.1111/j.1365-246X.2006.02967.x, 2006.

Sutherland, R., Basement geology and tectonic development of the greater New Zealand region: an interpretation from regional magnetic data, Tectonophysics, 308, 341-362, 1999a.

Sutherland, R., Cenozoic Bending of New Zealand Basement Terranes and Alpine Fault Displacement: a brief review, New Zealand Journal of Geology \& Geophysics, 42, 295-301, 1999b.

Takahashi, N., S. Kodaira, Y. Tatsumi, Y. Kaneda, and K. Suyehiro, Structure and growth of the Izu-Bonin-Mariana arc crust: 1. Seismic constraint on crust and mantle structure of the Mariana arc-back-arc system, Journal of Geophysical Research, 113(B1), 1-18, doi:10.1029/2007JB005120, 2008.

Takeuchi, H., and M. Saito, Seismic Surface Waves, pp. 217-295, 1988. 
Tape, C., Q. Liu, A. Maggi, and J. Tromp, Seismic tomography of the southern California crust based on spectral-element and adjoint methods, Geophyscial Journal International, 180, 433-462, doi:10.1111/j.1365-246X.2009.04429.x, 2010.

Tatsumi, Y., The subduction factory: How it operates in the evolving Earth, GSA Today, 15(7), 4, doi:10.1130/1052-5173(2005)015[4:TSFHIO]2.0.CO;2, 2005.

Townend, J., Heat flow through the West Coast, South Island, New Zealand, New Zealand Journal of Geology and Geophysics, 42, 21-31, 1999.

Townend, J., Y. Behr, K. Buckley, M. K. Savage, and J. Hine, A grid-based facility for large-scale cross-correlation of continous seismic data, EQC report, pp. i-29, 2009a.

Townend, J., Y. Behr, K. Buckley, M. K. Savage, and J. Hine, A Grid-Based Facility for Large-Scale Cross-Correlation of Continuous Seismic Data, in eResearch Australia, Sydney, 2009b.

Tsai, V. C., On establishing the accuracy of noise tomography travel-time measurements in a realistic medium, Geophysical Journal International, 178(3), 1555-1564, doi:10. 1111/j.1365-246X.2009.04239.x, 2009.

Tsai, V. C., The relationship between noise correlation and the Green's function in the presence of degeneracy and the absence of equipartition, Geophysical Journal International, 182(3), 1509-1514, doi:10.1111/j.1365-246X.2010.04693.x, 2010.

Villaseñor, A., M. H. Ritzwoller, A. L. Levshin, M. P. Barmin, E. R. Engdahl, W. Spakman, and J. Trampert, Shear velocity structure of central Eurasia from inversion of surface wave velocities, Physics of the Earth and Planetary Interiors, 123, 169-184, 2001.

Walcott, R., Modes of oblique compression: Late Cenozoic tectonics of the South Island of New Zealand, Reviews of Geophysics, 36(1), 1-26, 1998.

Walcott, R. I., The kinematics of the plate boundary zone through long-term deformations, Geophys. J. R. asp. Soc., 79, 613-633, 1984.

Wallace, L. M., Subduction zone coupling and tectonic block rotations in the North Island, New Zealand, Journal of Geophysical Research, 109(B12), 1-21, doi:10.1029/ 2004JB003241, 2004.

Wallace, L. M., et al., Characterizing the seismogenic zone of a major plate boundary subduction thrust: Hikurangi Margin, New Zealand, Geochemistry Geophysics Geosystems, 10(10), doi:10.1029/2009GC002610, 2009. 
Wapenaar, K., Green's function retrieval by cross-correlation in case of one-sided illumination, Geophysical Research Letters, 33(19), doi:10.1029/2006GL027747, 2006.

Wathelet, M., Array recordings of ambient vibrations: surface wave inversion, Ph.D. thesis, Faculté des Sciences Appliquées, 2005.

Wathelet, M., An improved neighborhood algorithm: Parameter conditions and dynamic scaling, Geophysical Research Letters, 35(9), doi:10.1029/2008GL033256, 2008.

Wathelet, M., D. Jongmans, and M. Ohrnberger, Surface-wave inversion using a direct search algorithm and its application to ambient vibration measurements, Near Surface Geophysics, 2, 211-221, 2004.

Wathelet, M., D. Jongmans, M. Ohrnberger, and S. Bonnefoy-Claudet, Array performances for ambient vibrations on a shallow structure and consequences over Vs inversion, Journal of Seismology, 12(1), 1-19, 2008.

Weaver, R. L., and O. I. Lobkis, Ultrasonics without a source: thermal fluctuation correlations at MHz frequencies, Physical Review Letters, 87, doi:10.1103/PhysRevLett.87. 134301, 2001a.

Weaver, R. L., and O. I. Lobkis, On the Emergence of the Green's Function in the Correlations of a Diffuse Field, Journal of the Acoustical Society of America, 110, 3011-3017, $2001 b$.

Weaver, R. L., and O. I. Lobkis, Diffuse fields in open systems and the emergence of the Green's function, Journal of the Acoustical Society of America, 116, 2731-2734, doi:10.1121/1.1810232, 2004.

Weaver, R. L., and O. I. Lobkis, Fluctuations in diffuse field-field correlations and the emergence of the Green's function in open systems, Journal of the Acoustical Society of America, 117, 3432-3439, doi:10.1121/1.1898683, 2005.

Wilson, C. J. N., The 26.5 ka Oruanui Eruption, Taupo Volcano, New Zealand: Development, Characteristics and Evacuation of a Large Rhyolitic Magma Body, Journal of Petrology, 47(1), 35-69, doi:10.1093/petrology/egi066, 2006.

Wilson, C. J. N., B. F. Houghton, M. McWilliams, M. Lanphere, S. Weaver, and R. Briggs, Volcanic and structural evolution of Taupo Volcanic Zone, New Zealand: a review, Journal of Volcanology and Geothermal Research, 68(1-3), 1-28, doi: 10.1016/0377-0273(95)00006-G, 1995. 
Wilson, C. K., C. H. Jones, P. Molnar, A. F. Sheehan, and O. S. Boyd, Distributed deformation in the lower crust and upper mantle beneath a continental strike-slip fault zone: Marlborough fault system, South Island, New Zealand, Geology, 32(10), 837840, 2004.

Yang, Y., and M. H. Ritzwoller, Characteristics of ambient seismic noise as a source for surface wave tomography, Geochemistry Geophysics Geosystems, 9(2), doi:10.1029/ 2007GC001814, 2008.

Yang, Y., M. H. Ritzwoller, A. L. Levshin, and N. M. Shapiro, Ambient noise Rayleigh wave tomography across Europe, Geophysical Journal International, 168(1), 259-274, doi:10.1111/j.1365-246X.2006.03203.x, 2007.

Yao, H., and R. D. Van Der Hilst, Analysis of ambient noise energy distribution and phase velocity bias in ambient noise tomography, with application to SE Tibet, Geophysical Journal International, 179(2), 1113-1132, doi:10.1111/j.1365-246X.2009.04329.x, 2009.

Yao, H., R. D. Van Der Hilst, and M. V. de Hoop, Surface-Wave Array Tomography in SE Tibet from Ambient Seismic Noise and Two-Station Analysis -I. Phase Velocity Maps, Geophysical Journal International, 166, 732-744, doi:10.1111/j.1365-246X. 2006.03028.x, 2006.

Yao, H., C. Beghein, and R. D. Van Der Hilst, Surface wave array tomography in SE Tibet from ambient seismic noise and two-station analysis - II. Crustal and uppermantle structure, Geophysical Journal International, 173, doi:10.1111/j.1365-246X. 2007.03696.x, 2008.

Yu, Y., and J. Park, Hunting for Azimuthal Anisotropy Beneath the Pacific Ocean Region, Journal of Geophysical Research, 99(B8), 15,399-15,421, 1994.

Zhang, H., C. Thurber, and P. Bedrosian, Joint inversion for $\mathrm{Vp}, \mathrm{Vs}$, and $\mathrm{Vp} / \mathrm{Vs}$ at SAFOD, Parkfield, California, Geochemistry Geophysics Geosystems, 10(11), 1-17, doi:10.1029/2009GC002709, 2009.

Zhang, J., P. Gerstoft, and P. D. Bromirski, Pelagic and coastal sources of P -wave microseisms: Generation under tropical cyclones, Geophysical Research Letters, 37(15), 1-6, doi:10.1029/2010GL044288, 2010.

Zhou, Y., G. Nolet, F. A. Dahlen, and G. Laske, Global upper-mantle structure from finitefrequency surface-wave tomography, Journal of Geophysical Research, 111(B4), 1-24, doi:10.1029/2005JB003677, 2006. 
Zielhuis, A., and G. Nolet, Deep seismic expression of an ancient plate boundary in Europe, Science (New York, N.Y.), 265(5168), 79-81, doi:10.1126/science.265.5168.79, 1994. 